\title{
WestVirginiaUniversity
}

THE RESEARCH REPOSITORY @ WVU

Graduate Theses, Dissertations, and Problem Reports

2014

\section{Advanced Composites for Design and Rehabilitation of Hydraulic Structures}

Piyush Ram Soti

West Virginia University

Follow this and additional works at: https://researchrepository.wvu.edu/etd

\section{Recommended Citation}

Soti, Piyush Ram, "Advanced Composites for Design and Rehabilitation of Hydraulic Structures" (2014). Graduate Theses, Dissertations, and Problem Reports. 642.

https://researchrepository.wvu.edu/etd/642

This Thesis is protected by copyright and/or related rights. It has been brought to you by the The Research Repository @ WVU with permission from the rights-holder(s). You are free to use this Thesis in any way that is permitted by the copyright and related rights legislation that applies to your use. For other uses you must obtain permission from the rights-holder(s) directly, unless additional rights are indicated by a Creative Commons license in the record and/ or on the work itself. This Thesis has been accepted for inclusion in WVU Graduate Theses, Dissertations, and Problem Reports collection by an authorized administrator of The Research Repository @ WVU. For more information, please contact researchrepository@mail.wvu.edu. 


\title{
Advanced Composites for Design and Rehabilitation of
}

\section{Hydraulic Structures}

\author{
Piyush Ram Soti
}

Thesis submitted to the

Benjamin M. Statler College of Engineering and Mineral Resources at

West Virginia University in

partial fulfillment of the requirements

for the degree of

\section{Master of Science \\ in \\ Civil Engineering}

\author{
P V. Vijay, Ph.D, PE, Chair \\ Hota V. GangaRao, Ph.D, PE \\ Udaya B. Halabe, Ph.D, PE
}

Department of Civil and Environmental Engineering

Morgantown, West Virginia

2014

Keywords: FRP, underwater wrapping, Aquawrap, rehabilitation, ceramic coatings, abrasion Copyright 2014 Piyush Ram Soti 


\section{ABSTRACT \\ Advanced Composites for Design and Rehabilitation of Hydraulic Structures \\ Piyush Ram Soti \\ West Virginia University}

Concrete and steel have been predominantly used in civil and hydraulic infrastructure construction. With several years of service under adverse climatic conditions, those structures have exhibited moderate to severe deterioration and composite materials are emerging as one of the most feasible and economical materials for their rehabilitation. This report describes and highlights the research work conducted on: i) rehabilitation of underwater concrete structures with FRP, and ii) evaluation of abrasion resistant coatings for hydraulic steel gates.

Curing of certain class of resins under water has unfolded the possibility of utilizing fiber reinforced polymers (FRPs) to repair submerged piles and pier structures. In this study, GFRP pre-preg with water curable urethane resin was used to wrap concrete cylinders. Laboratory testing shows the increase in strength, stiffness, and ductility of concrete cylinders with underwater FRP wrapping. Lab evaluation also indicates that underwater FRP system is effective in dry surface wrapping (ambient field conditions) as well. Threefold increase in load capacity (1921 psi vs. 6865 psi) and 3.22 times increment in energy absorption was observed with 5 layers of wrapping. Concrete cylinders with steel sections and wrapped with 2 layers of Aquawrap also showed significant increase in load capacity with more than $92 \%$ degree of compositeness. By applying basic confinement effect equations, theoretical strengths were in good correlation with experimental values. Following lab work, the rehabilitation work was conducted on submerged concrete discharge ports of Chickamauga lock and dam, and steel piles of East Lynn Lake Bridge.

This research also focuses on the study of abrasion characteristics and comparative abrasion tests on different abrasion resistant coating systems. Conditions of severe abrasion exist at some high-lift dams that cause standard protective coatings on the downstream side of tainter gates to be abraded away in just few years of service. Down-stream side of protective coating system is damaged typically by debris that swirls and bangs against the gate causing severe cutting and abrasion to the coatings. This study was aimed at identifying composite based coating that can withstand hydraulic forces from high velocity water and also minimize the effects of severe cutting, impact, and abrasive forces generated from barge and sand particles on tainter gates at Heflin Lock \& Dam near Gainesville, Alabama. In this research, six different types of abrasion resistant coating materials/systems were applied on sand blasted metallic plates in order to perform comparative abrasion tests using TABER reciprocating abrader. Among six different coating systems, ceramic composite based coatings provided better performance (up to twice or better) over the conventional coatings. 


\section{ACKNOWLEDGEMENTS}

I would like to thank my advisor, Dr. P.V. Vijay for nurturing me throughout my studies and research. His support and valuable insights were extreme motivations for me to finish my Master's degree.

I am also grateful to Dr. Hota GangaRao for giving me the opportunity to study as a graduate student at WVU and simultaneously work at Constructed Facilities Center (CFC-WVU) as a research assistant. I thank him for his motivation, support and guidance.

I am also extremely thankful to Dr. Udaya B. Halabe for giving me constant feedback during my studies at WVU. I pay my sincere gratitude to him for being a member of my review committee and helping me throughout my stay here at WVU.

I would also like to express my sincere appreciation to Mark Skidmore for teaching me to set up instruments and acquire data from them. He was always ready to help me with the testing and provide his invaluable insights. I would also like to thank Jerry Nestor for helping me in the structures lab, without whom, I could have never known to begin and proceed with the tests. He is such a crucial member for not only me but also with all the students working in the lab.

I am also extremely thankful to Tyler beaty. Tyler was the one to push me early in the mornings to start our work in the lab. We did all our work together helping each other. He has helped me and supported me and this thesis would not have been completed without him.

Finally, I thank all my friends here in Morgantown who made me feel at home and for being the ones with whom I was able to share my happiness and sadness. I also thank my family for everything. Without them, I could not have accomplished this degree. 


\section{TABLE OF CONTENTS}

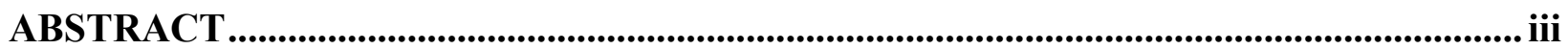

ACKNOWLEDGEMENTS ......................................................................................................

TABLE OF CONTENTS ….......................................................................................................... iv

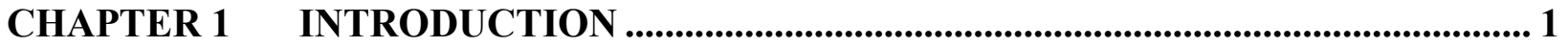

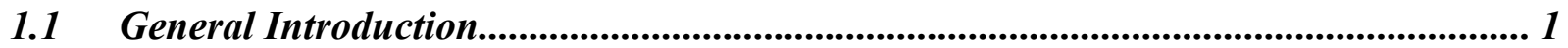

1.2 Objectives.............................................................................................................................. 4

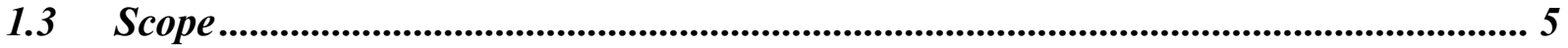

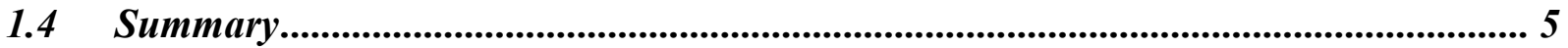

CHAPTER 2 LITERATURE REVIEW _.................................................................... 7

$2.1 \quad$ Introduction ........................................................................................................................... 7

$2.2 \quad$ FRP Confinement of Columns ......................................................................................... 7

2.2.1 Underwater FRP Strengthening of Columns ..................................................... 9

$2.3 \quad$ Abrasion and Wear Mechanism ................................................................................... 13

2.3.1 Wear Resistance and hardness of the Materials..................................................... 15

2.3.2 Surface Wettability on Friction and Wear ............................................................ 16

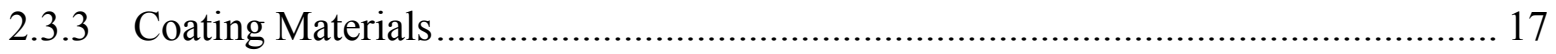

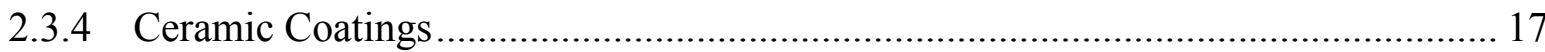




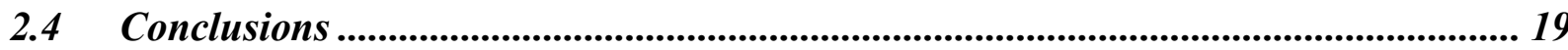

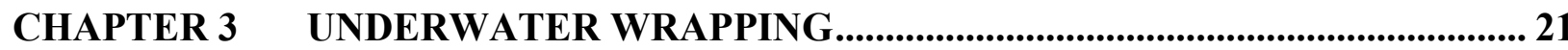

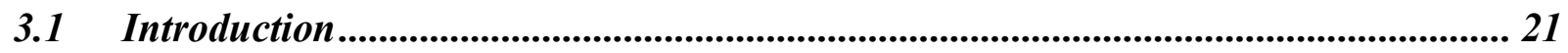

$3.2 \quad$ Materials and Properties............................................................................................ 22

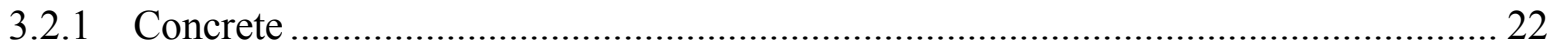

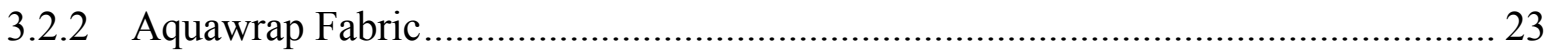

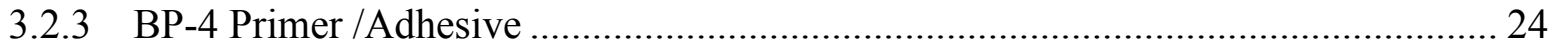

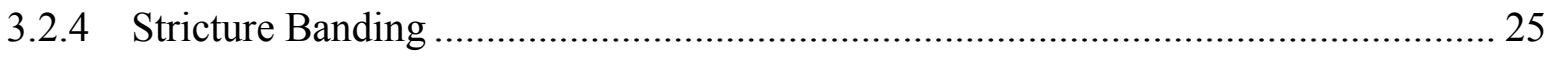

$3.3 \quad$ Wrapping Details........................................................................................................... 26

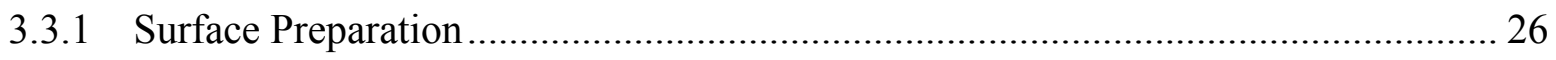

3.3.2 Application of Base Primer\#4 (BP-4) Adhesive/Primer........................................... 27

3.3.3 Installation of Aquawrap around the Concrete Cylinder .......................................... 28

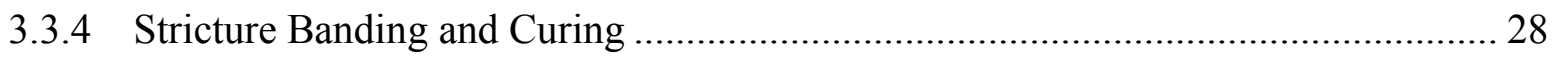

$3.4 \quad$ Experimental Methods on Cylinders \& Wraps ................................................................. 29

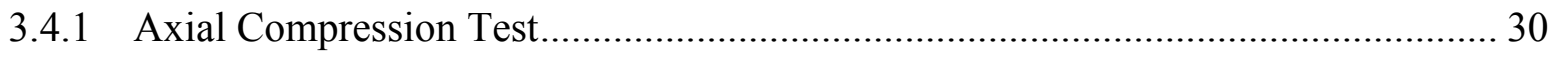

3.4.2 Tension Test on FRP Strip Specimens............................................................... 30

$3.5 \quad$ Results and Discussions ............................................................................................... 31

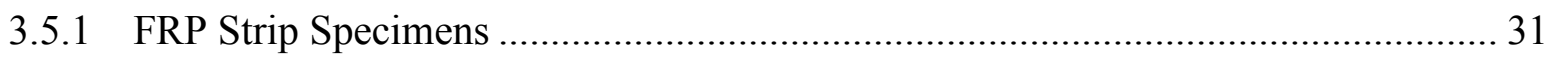

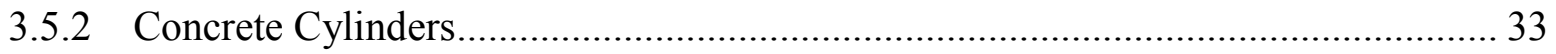

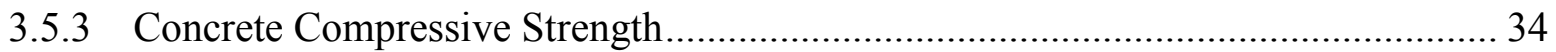




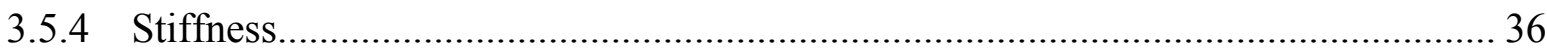

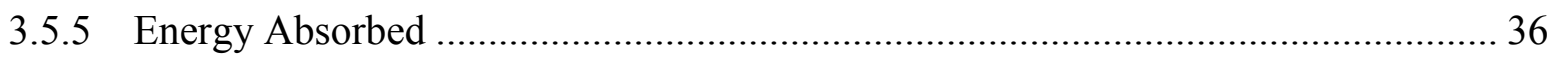

$3.6 \quad$ Theoretical Analysis.......................................................................................................... 38

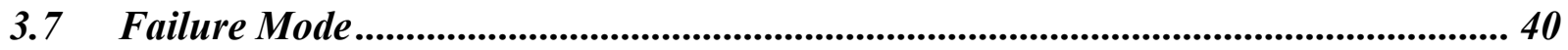

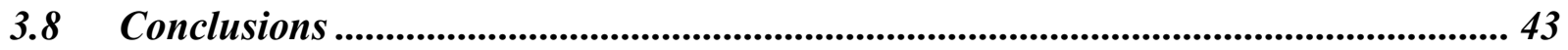

\section{CHAPTER 4 REHAB OF CONCRETE DISCHARGE PORTS IN CHICKAMAUGA}

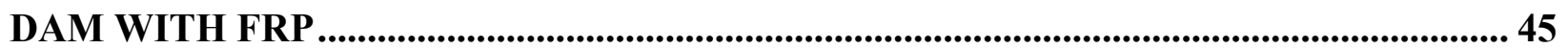

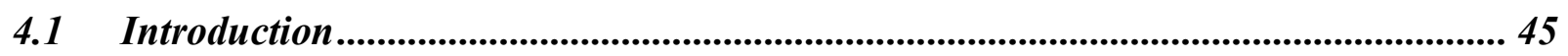

$4.2 \quad$ Field Installation ............................................................................................................ 48

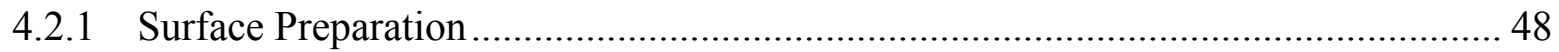

4.2.2 Wrapping of the Discharge Ports ............................................................................ 49

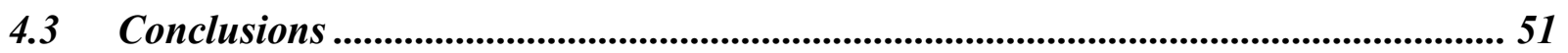

CHAPTER 5 REHAB OF STEEL PILES IN EAST LYNN LAKE BRIDGE.................52

$5.1 \quad$ Introduction .......................................................................................................................... 52

$5.2 \quad$ Initial Lab Testing............................................................................................................. 53

5.2.1 Strength of Wrapped Cylinders without Steel Columns ............................................ 54

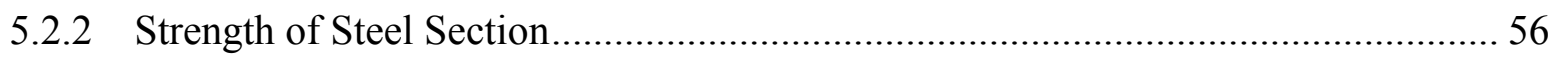

5.2.3 Strength of Wrapped Cylinders with Steel Columns …………………………....... 56

5.2.4 Strength of Wrapped Cylinders with Steel Columns having Shear Transfer

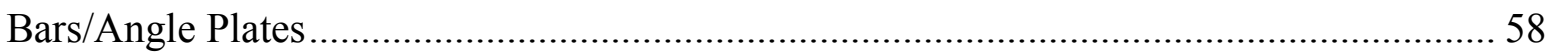


5.2.5 Summary

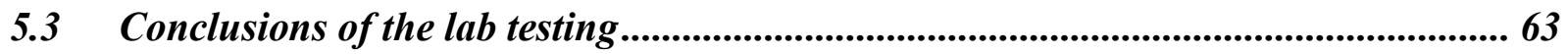

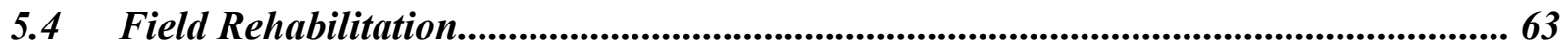

5.5 Strength calculations for rehab of steel H-piles/columns ...................................... 69

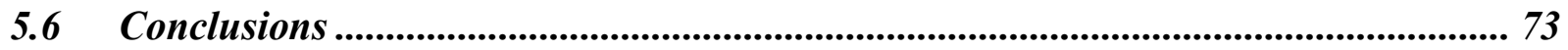

CHAPTER 6 ABRASION RESISTANT COATINGS .............................................. 74

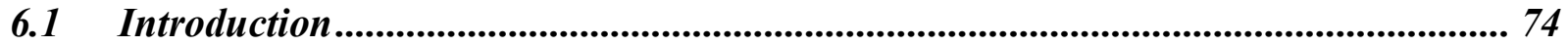

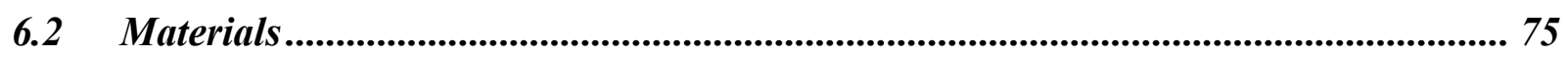

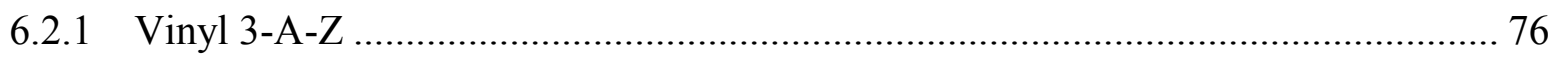

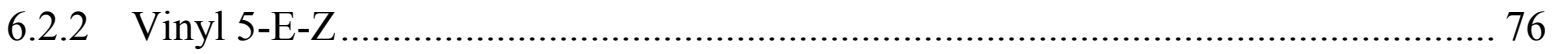

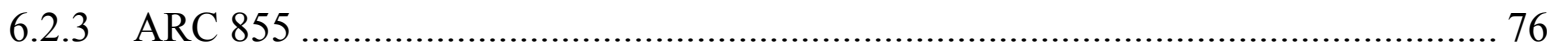

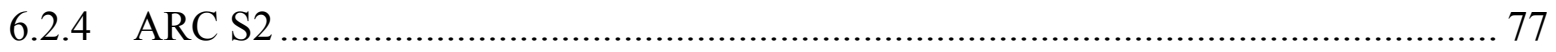

6.2.5 UHMW-PE (Ultra high-molecular weight polyethylene) ................................. 77

6.2.6 Flexible (Fibrous) Polymer Coating ........................................................... 78

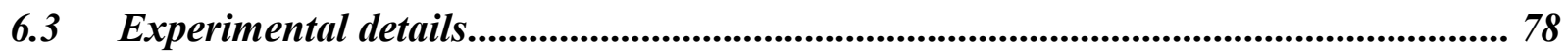

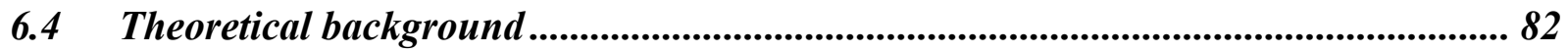

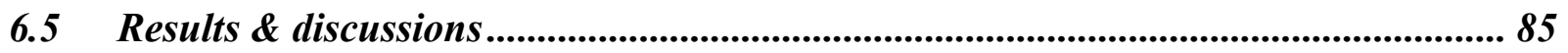

6.6 Wear rate for different coatings................................................................... 94

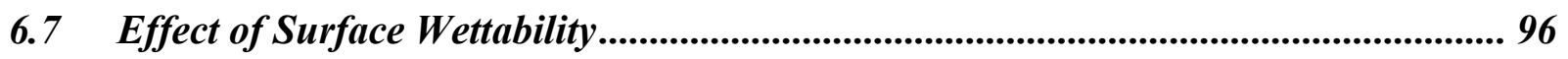

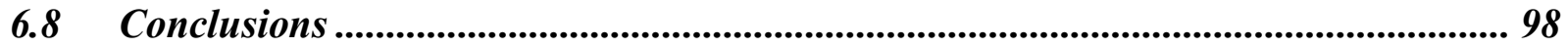




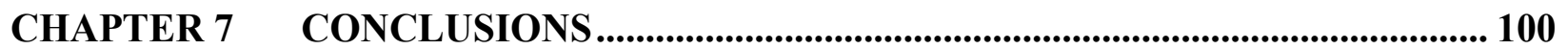

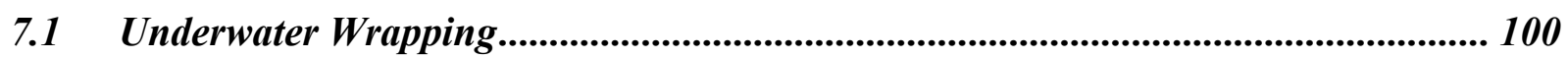

7.2 Rehabilitation of Concrete Discharge Ports in Chickamauga Dam .......................... 101

7.3 Rehabilitation of Steel Piles in East Lynn Lake Bridge ............................................... 101

$7.4 \quad$ Abrasion Resistant Coatings........................................................................................... 102

7.5 FRP Miter Block and FRP Recess Panel...................................................................... 104

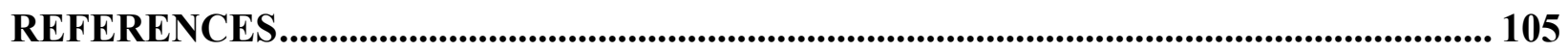

APPENDIX A: Application of FRP Wraps and Coatings ............................................................ 109

APPENDIX B: FRP Miter Blocks ................................................................................................... 111

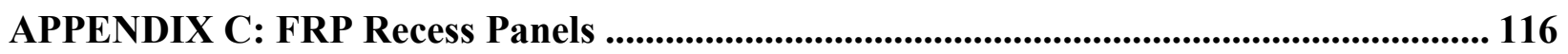




\section{LIST OF FIGURES}

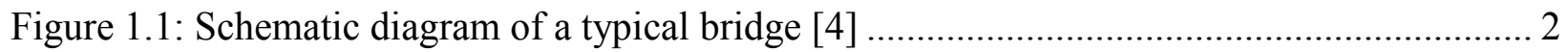

Figure 1.2: Corrosion on tainter gates at Heflin dam (left), close-up view of corroded tainter

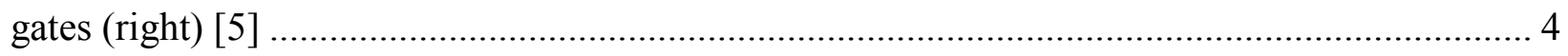

Figure 2.1: Schematic diagram of section of the confined concrete [7] ..................................... 8

Figure 2.2: Lateral expansion (left), effect of confinement (right) [7] ….................................... 9

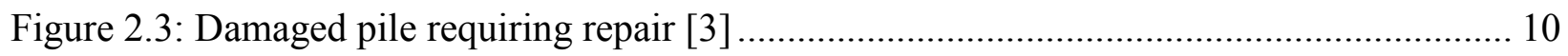

Figure 2.4: Concrete encapsulation (left) and Aquawrap installation (right) [8] ........................ 11

Figure 2.5: Flexural testing of FRP attached under the beam [14] ........................................ 13

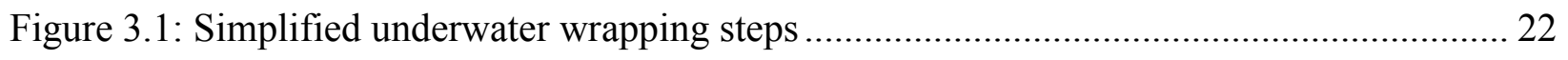

Figure 3.2: Casting of concrete (left) and curing of concrete (right) ........................................... 23

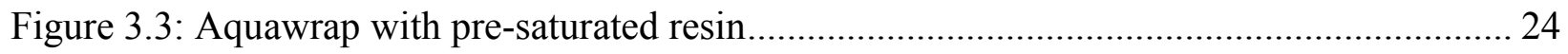

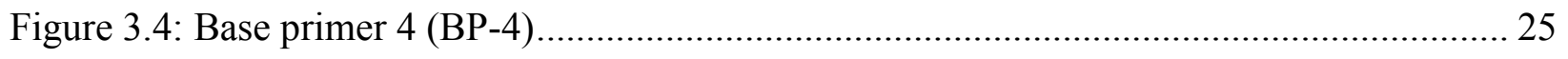

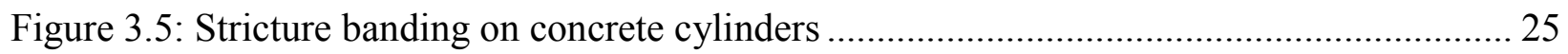

Figure 3.6: Concrete cylinder, steel disc brush, concrete grinding discs..................................... 27

Figure 3.7: Underwater application of BP-4 primer (left) and Aquawrap on concrete cylinder

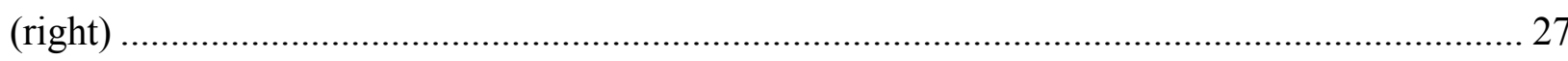

Figure 3.8: Aquawrap opened from the pouch (left) and underwater wrapping of cylinders (right)

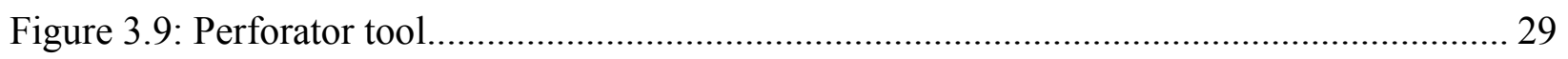

Figure 3.10: Stretching Stricture banding (left) and Curing of wrapped cylinders (right) ........... 29

Figure 3.11: Axial compression test of wrapped cylinder on Instron 1000HDX ……................. 30 
Figure 3.12: Testing of Aquawrap strip on Instron 8501 (left) \& test strip attached with

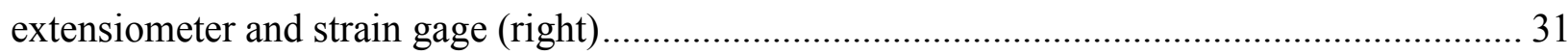

Figure 3.13: Load vs. Strain plots for 12"X1" single layer strip ......................................... 32

Figure 3.14: Average axial strength increase for 1, 2, 3, and 5 layers of wrapping .................. 35

Figure 3.15: Load vs. axial deformation graphs for cylinders with 1, 2, 3 and 5 wraps ........... 37

Figure 3.16: Stress vs. axial, hoop strains for 1, 2, 3, and 5 wraps..................................... 38

Figure 3.17: Failure mode of wrapped concrete cylinders with 1, 2, 3 and 5 layers of wrapping 42 Figure 3.18: Failure mode of wrapped cylinders (five star structural concrete) with 3 and 5 layers

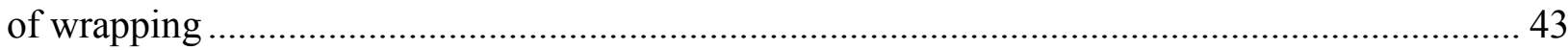

Figure 4.1: Old lock (60 ft. x $360 \mathrm{ft}$.) and new lock (110 ft. x $660 \mathrm{ft}$.) in Chickamauga dam, TN 45

Figure 4.2: Plan view of discharge ports at Chickamauga lock and dam, TN........................ 47

Figure 4.3: Selected view of discharge ports …........................................................ 47

Figure 4.4: View of water released through discharge ports at the Chickamauga lock and dam. 48 Figure 4.5: Horizontal and vertical sections of discharge ports at Chickamauga lock and dam, TN

Figure 4.6: Boats with rehab materials, crew, diving support and monitoring system; Resins

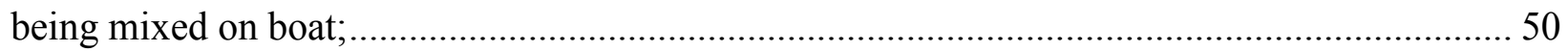

Figure 4.7: Resin saturated FRP fabric roll being passed onto diving team member, Onboard

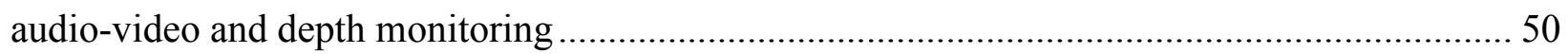

Figure 4.8: Hand application of primer on discharge ports ............................................... 50

Figure 5.1: East Lynn Lake Bridge (1969) with severely corroded steel structural members, Cove Creek, WV (left); close-up of corroded steel pile (right)................................................. 53 
Figure 5.2: Fast-setting concrete, Aquawrap, and steel section S4x 7.7 .............................. 54

Figure 5.3: Cast concrete cylinders and wrapped concrete cylinders .................................... 54

Figure 5.4: Stress vs. strain plot for 6"x12" concrete cylinder.......................................... 55

Figure 5.5: Failure of steel member in compression........................................................... 56

Figure 5.6: Load vs. strain plot for concrete cylinder with steel inside and 2 layers of Aquawrap

Figure 5.7: Failure of concrete cylinders with steel member and 2 layers of wrap ................... 58

Figure 5.8: Different variations on steel member (V1, V2, V3, and V4) (left to right) ............. 59

Figure 5.9: Load vs. strain plots for different variations on steel members ............................ 60

Figure 5.10: Failure of the wrapped concrete cylinders with steel inside ............................... 62

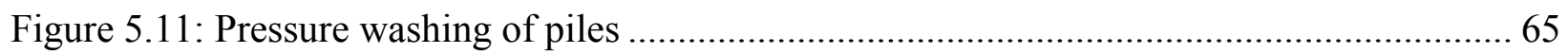

Figure 5.12: Scaffolding placed around the piles (left) and shear transferring rods placed (right)

Figure 5.13: Placement of FRP jackets around the pile and gluing the joint............................ 66

Figure 5.14: Mixing of polymer grout and placing them into the FRP shell around the pile....... 67

Figure 5.15: Stacking of FRP shells and placing the wooden box for pouring concrete............. 67

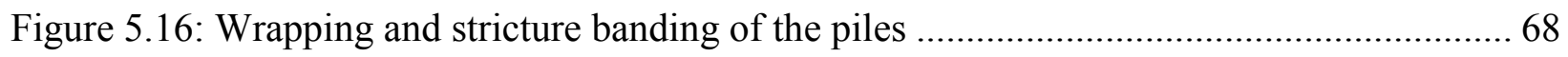

Figure 5.17: Pouring of concrete and stricture banding on the top portion of the piles .............. 68

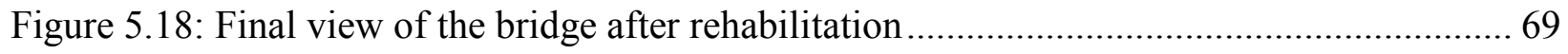

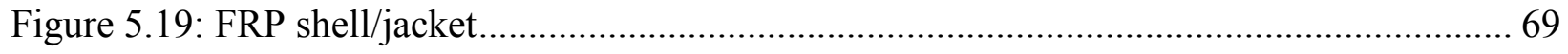

Figure 5.20: FRP strip cut from circumferential direction (left) \& from longitudinal direction

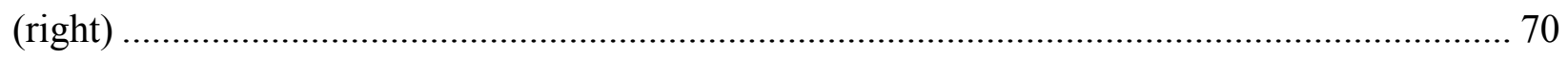

Figure 5.21: Rehabilitated pile geometry.................................................................. 71 
Figure 6.1: Debris that swirls around on the downstream side of the tainter gate (left); damage to the vinyl coating system caused by swirling debris (right) [5] .......................................... 75

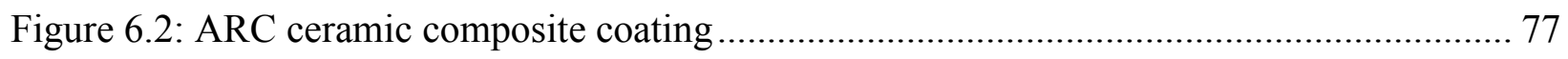

Figure 6.3: Dimension of the metal plate and locations (L1, L2, L3) of Scanning .................... 79

Figure 6.4: GL-7 PRO, TABER reciprocating abrader, profilometer (from left to right) ........... 79

Figure 6.5: Abraded coating specimens under no conditionings (i.e. dry surface) ................... 79

Figure 6.6: Abraded coating specimens under water immersion conditions (i.e. wet surface) .... 80

Figure 6.7: Abrasion on coating surface of vinyl 3-a-z, vinyl 5-e-z, ARC 855, flexible polymer,

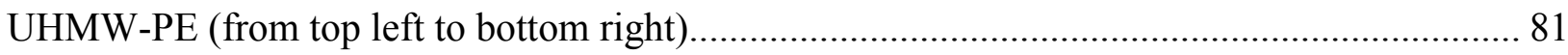

Figure 6.8: Wear down of the specimen surface during abrasion with tribological intensity $\psi$ [36] 84

Figure 6.9: Depth profile of vinyl 3-a-z coating after 50,000 cycles of abrasion (dry surface, wet

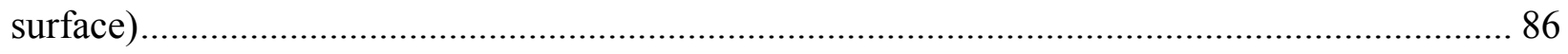
Figure 6.10: Depth profile of vinyl 5-e-z coating after 50,000 cycles of abrasion (dry surface, wet

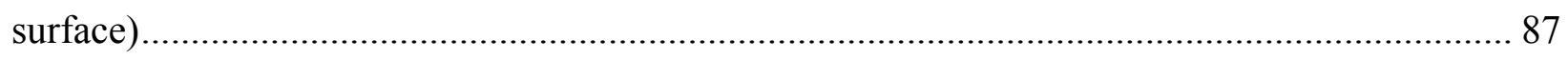
Figure 6.11: Depth profile of ARC 855 coating after 50,000 cycles of abrasion (dry, wet surface) 88

Figure 6.12: Depth profile of ARC S2 coating after 50,000 cycles of abrasion (dry, wet surface) 89

Figure 6.13: Depth profile of UHMW-PE coating after 50,000 cycles of abrasion (dry, wet surface) 90

Figure 6.14: Depth profile of Rutgers coating after 50,000 cycles of abrasion (dry surface, wet surface). 91 
Figure 6.15: Abraded volume of coatings after different cycles of abrasion under dry/wet

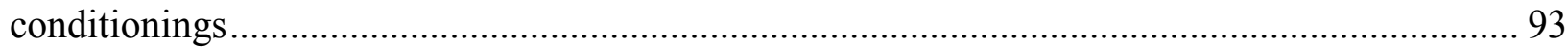

Figure 6.16: Abraded volume of coatings after different cycles of abrasion under dry/wet

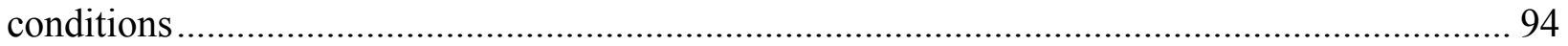

Figure 6.17: Abraded volume vs. sliding distance $\mathrm{x}$ load for different coatings ...................... 95

Figure 6.18: Water drop and its contact angles on different coating materials ........................ 97 


\section{LIST OF TABLES}

Table 2-1: Factors influencing abrasive wear behavior [18] ........................................... 14

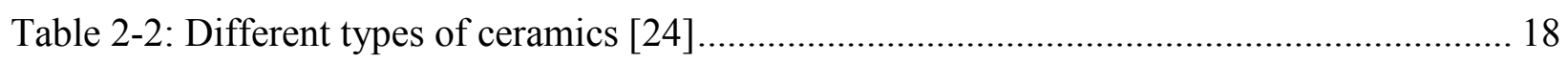

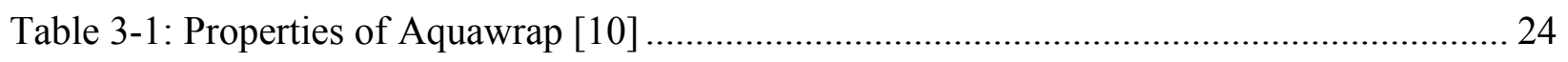

Table 3-2: Properties of base primer/adhesive (BP-4) [26] ............................................. 25

Table 3-3: Axial tensile strength of 304.8 mm X 25.4 mm (12” X 1”) Aquawrap strips............ 32

Table 3-4: Strength of non-wrapped/wrapped concrete cylinders \& their \% increase in strength 33

Table 3-5: Strength \& \% strength increase of non-wrapped/wrapped cylinders cast with

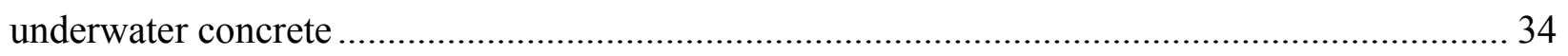

Table 3-6: Energy absorbed by concrete cylinders until failure ........................................... 37

Table 3-7: Analytical models used for correlation ......................................................... 39

Table 3-8: Comparisons of experimental \& theoretical confined strength of 4" diameter concrete

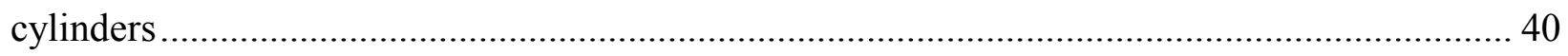

Table 5-1: Strength of 6"x12" non-wrapped/wrapped concrete cylinders ............................... 55

Table 5-2: Strength of steel member (S4x7.7) with c/s area of 2.26 in2 ............................... 56

Table 5-3: Strength of concrete cylinders with steel S4x7.7 inside and 2 layers of G-05

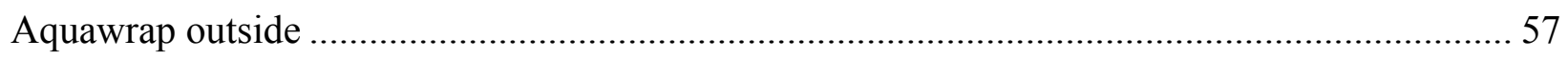

Table 5-4: Different variations on steel member and their corresponding load to failure........... 59

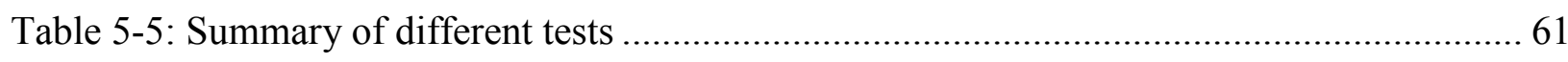

Table 5-6: Tensile stress of 2\# of Aquawrap strip ......................................................... 70

Table 5-7: Tensile stress of strips cut from FRP shell ................................................... 70

Table 5-8: Compressive strength of 4" x 8" Self-Consolidated Concrete (SCC) cylinders ......... 70

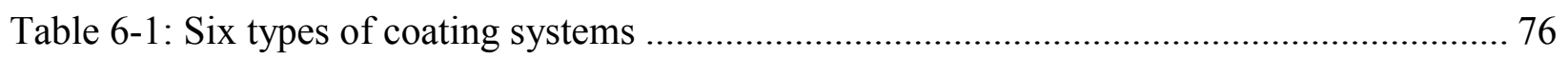


Table 6-2: Thicknesses of different coatings on the metal plates .......................................... 80

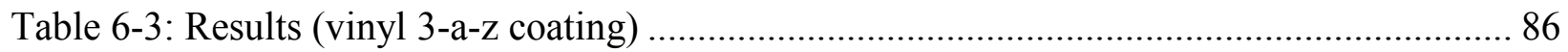

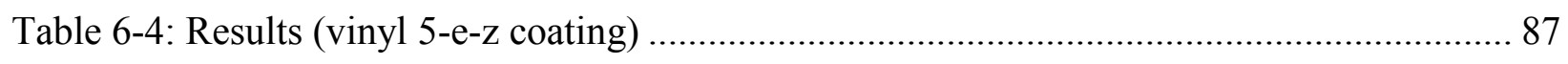

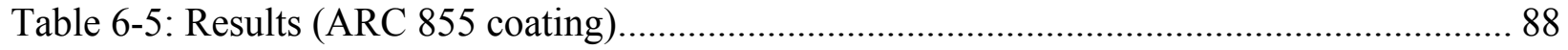

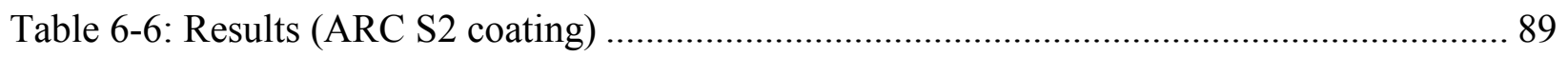

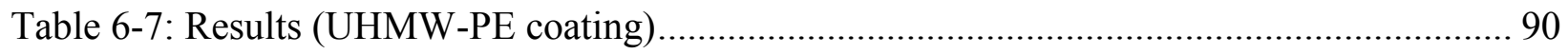

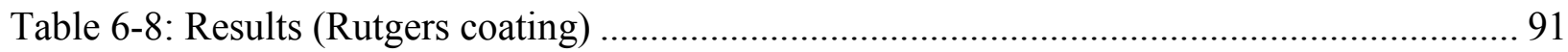

Table 6-9: Abraded volume of the scar on both dry and wet surface of the coatings ................ 92

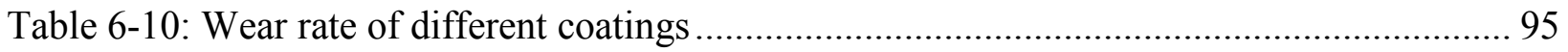

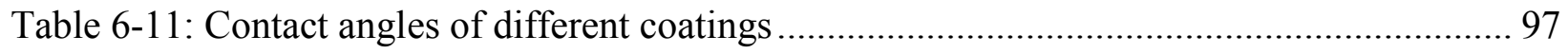




\section{CHAPTER 1 INTRODUCTION}

\subsection{General Introduction}

America has made huge investments in basic infrastructures such as like roads, bridges, dams, canals etc. including the groundbreaking transportation system "Interstates" being started in 60's and 70's. These basic infrastructures provided the foundations for America's future growth and advancement [1]. Roads and Bridges have evolved over time into modern transportation system capable of connecting the nation and carrying vast amounts of goods and services. Safe operation of these civil infrastructure systems is vital to our nation's prosperity [1]. According to ASCE report card for America's infrastructures, more than $30 \%$ of existing bridges have exceeded their design life and America needs to spend $\$ 20.5$ billion annually to meet the nation's bridge deficiency backlogs by 2028 [2]. The failure of these structures will lead to the downfall of American productivity and economy. Therefore, researchers and engineers are constantly trying to find innovative solutions to prevent the collapse of those infrastructures and extend their longevity, to maintain their integrity and even to enhance their existing load capacities in a quick and economical manner.

Steel and concrete are conventionally used in the construction of infrastructure. These materials undergo deterioration when exposed to adverse environments. Steel reinforcements and shapes used in bridges, buildings, hydraulic structures, pavements and others undergo continued corrosion under constant exposure to natural environment. Concrete, on the other hand, undergoes cracking due to fatigue, creep, moisture etc. Concrete also deteriorates due to chemical reaction of its ingredients resulting in the reduction of strength, modulus, and load 
capacity, and further facilitating the corrosion of internal steel reinforcement. At present, there is a great need for repair and replacement of several of our aging structural systems.

Instead of replacing distressed bridges or hydraulic structures and spending billions of dollars, it is necessary to understand, if only rehabilitation and retrofit of these structures can extend their service life in lieu of new construction. At present, there are several advanced techniques available for rehabilitating and retrofitting old structures. Traditionally, rehabilitation of existing structures was done using steel or reinforced concrete jacketing or plate bonding. Currently, concrete structures are externally wrapped with FRP (Fiber Reinforced Polymer) composites. The use of FRP composites for rehabilitation has gained popularity because it provides significant amount of lateral confinement and enhances the axial strength, stiffness and energy absorption of wrapped structures. FRPs are being employed in many structural applications both for new construction or rehabilitation of distressed civil engineering infrastructure. Its popularity is due to its high strength to weight ratio, good durability, better corrosion-chemical-heat-resistance and manufacturability in any practical length and shape. As fibers/fabrics can be oriented in any direction, FRPs are excellent for emergency repairs [3].

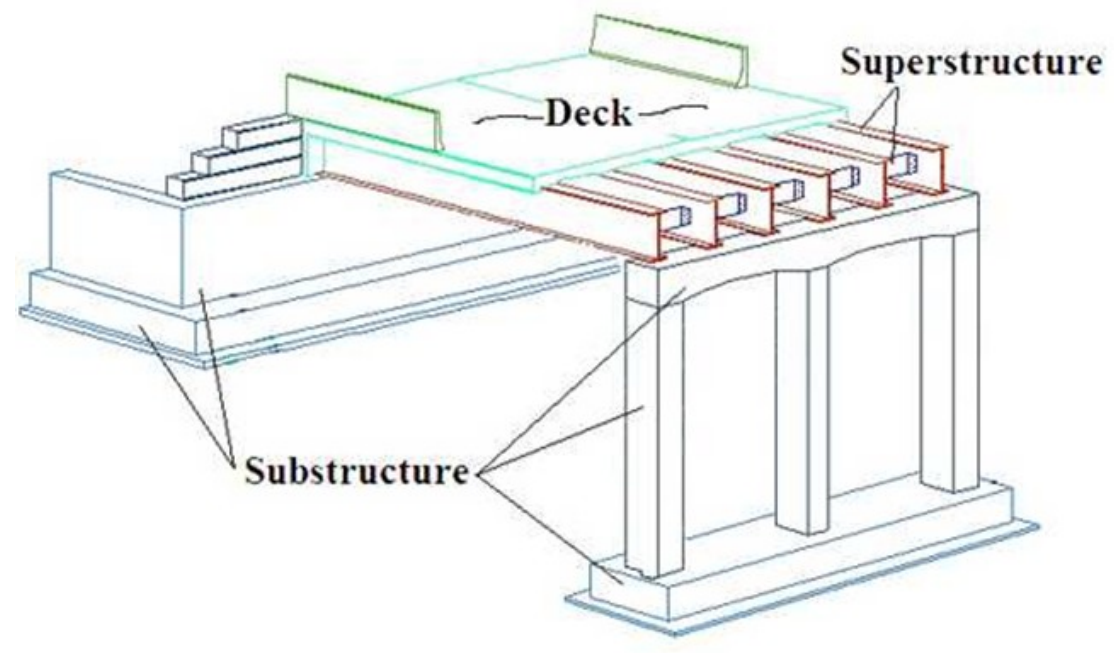

Figure 1.1: Schematic diagram of a typical bridge [4] 
Figure 1.1 shows the schematic diagram of a bridge with superstructure and substructure elements (supposing it as an old and in need of repair). Horizontal beams of the superstructure support deck and transfer the load into the substructure and this substructure takes all the vertical and lateral loads acting on them. Depending on the load and exposure conditions, superstructure and substructure elements deteriorate after certain years in service needing repair. Repair of those structures are necessary to ensure user safety by restoring or increasing their load carrying capacity. Wrapping these structures with FRP wraps are considered one of the easier methods to increase their load capacity. Until last few years, wrapping of these substructures with FRP wraps were done by constructing the cofferdams (a temporary watertight enclosure for repairs) around them. But now, due to the availability of resins that can cure under water, substructures completely submerged in water can be wrapped without the need of cofferdams. This research evaluates the feasibility and field implementation of underwater GFRP (Glass - Fiber Reinforced Polymer) wrapping and evaluates the confinement related strength, stiffness and ductility increase in concrete members.

Similar to bridges and buildings, hydraulic infrastructure also undergo deterioration under applied loads and environmental conditions. Figure 1.2 shows the corroded steel tainter gates at Heflin dam in Alabama. Such corroded steel members are also in need of repair to prevent sectional loss and the catastrophic failure. This research also evaluates the repair of corrosion of hydraulic structural steel gates which have been deteriorated due to abrasion from swirling current of water containing debris and sand particles. This study evaluates the application of suitable abrasion resistant coatings to prevent abrasion damage and corrosion of steel gates to enhance their longevity. 

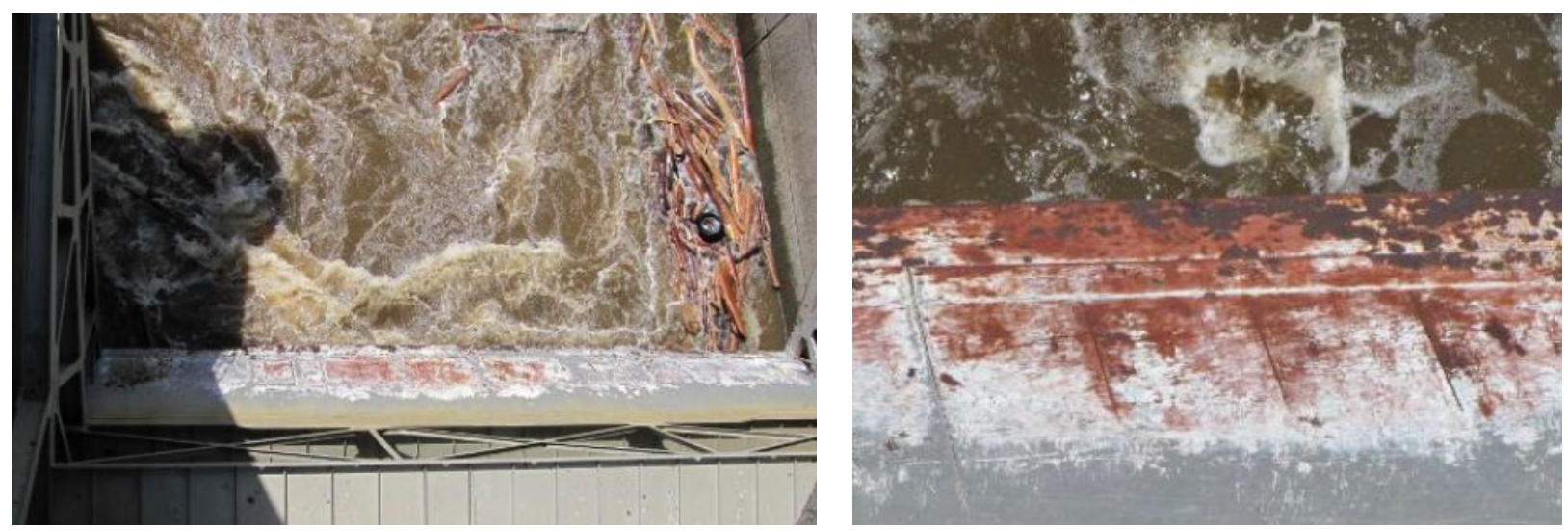

Figure 1.2: Corrosion on tainter gates at Heflin dam (left), close-up view of corroded tainter gates (right) [5]

\subsection{Objectives}

The objectives of this US Army Corps of Engineers (USACE) funded research are as follows:

1. To evaluate the feasibility of field implementation of underwater FRP composite repair systems for water immersed concrete discharge ports on the Chickamauga dam in Tennessee. The discharge ports which are similar to pier-like structures are in need of repair and retrofit to counter further loss of concrete under high velocity of flowing water.

2. To evaluate rehabilitation of the East Lynn Lake bridge steel piles/columns deteriorated due to severe corrosive environments using FRP shells and wraps with concrete filling.

3. To demonstrate the wear and abrasive mechanism on coatings.

4. To evaluate and compare currently applied coating and other systems for tainter gates at Heflin dam, Alabama, and to identify composite based coating systems that can better withstand abrasive action of flowing water and debris in order to protect steel tainter gate surface against corrosion and coating erosion. 


\subsection{Scope}

1. In order to meet the first objective, thirty six (36) plain concrete cylinders were cast with low strength concrete and twelve (12) plain concrete cylinders were cast with underwater rapid set structural mortar. Wrapping of these cylinders were done with 1, 2, 3 and 5 number of Aquawrap layers (pre-preg underwater wrap). Wrapped and non-wrapped concrete cylinders were subjected to axial compression in order to study the effect of confinement.

2. In order to meet the second objective, fifteen (15) cylinders were cast, with/without steel shapes (S4x7.7), which were further wrapped with 2 layers of Aquawrap. The axial load capacities were determined and the effects of confinement and compositeness were studied. It followed the field rehabilitation of steel piles at East Lynn Lake Bridge.

3. In order to meet the third and fourth objective, six (6) different abrasion resistant coating systems were evaluated by abrading their surfaces with Taber reciprocating abrader. The abraded scar volume/scar depth was measured and plotted using profilometer. Further, contact angles on those coatings were measured in order to correlate the wettability and abrasion.

\subsection{Summary}

This report is organized into 7 chapters, each dealing with particular repair, rehabilitation or coating systems of the topic. Chapter 1 provides general introduction, objectives and scope of this research. Chapter 2 presents the literature review on underwater wrapping, its confining effects and abrasion resistant coatings. Chapter 3 deals with the research related to underwater wrapping of concrete including materials, test set up, procedures, results, analytical models \& 
conclusions. Chapter 4 provides details on the field implementation of underwater wrapping for rehabilitating concrete discharge ports in Chickamauga dam, TN. Chapter 5 describes rehabilitation of steel piles in East Lynn Lake Bridge, WV. Chapter 6 deals with the research on abrasion resistant coatings including different coating systems, their application, abrasion tests on coatings and research results and analysis. Chapter 7 summarizes the conclusions including future recommendations. References and appendices are provided at the end of this report. The appendix also provides test results for FRP recess panel tested to evaluate its load distribution property and FRP miter block evaluated to identify its thermal characteristics. 


\section{CHAPTER 2 LITERATURE REVIEW}

\subsection{Introduction}

This chapter presents the overview of the FRP wrapping of above and under-water columns and wear mechanism of coatings used in hydraulic structures for preventing corrosive and abrasive actions of flowing water and debris.

\subsection{FRP Confinement of Columns}

Fiber reinforced polymer composites have found increasingly wide applications in civil engineering due to their high strength-to-weight ratio, better heat-chemical-and-corrosionresistance, better durability and good tailor ability. One of the important applications of FRPcomposites is confinement of the concrete, in both the retrofit of existing reinforced concrete (RC) columns by the provision of an FRP jacket or FRP wraps, and in concrete-filled FRP tubes in new construction. As a result of FRP confinement, both the compressive strength and ultimate strain of concrete can be greatly enhanced [6]. Columns wrapped with FRP sheets result in enhanced flexural and shear capacity, improved ductility as well as increased ultimate load carrying capacity.

The lateral confinement provided by FRP wraps results in better strength, stiffness and ductility of the FRP wrapped concrete. When concrete is subjected to axial compression, it expands laterally in hoop (circumferential) direction. This expansion is confined by FRP wraps, which is loaded in tension in the hoop direction, thus enhancing the load carrying capacity of concrete as illustrated in Figure 2.1 and 2.2, where all stresses are shown in their positive 
directions. In concrete, compressive stresses and strains are defined as positive but in the FRP, tensile stresses and strains are positive.

The lateral (radial) confining pressure acting on the concrete core $\left(f_{l}\right)$ is given by Equation 2.1.

$$
f_{l}=\frac{f_{f r p u} t_{f r p}}{r}=\frac{2 f_{f r p u} t_{f r p}}{d}
$$

Where,

$f_{\text {frpu }}=$ Tensile stress in the FRP jacket in the hoop direction,

$t_{f r p}=$ Total thickness of the FRP jacket, and

$r \& d=$ Radius and diameter of the confined concrete core, respectively.

If the FRP is loaded in hoop tension only, then the hoop stress in the FRP jacket $f_{f r p u}$ is proportional to the hoop strain $\epsilon_{f r p u}$ (due to the linearity of FRP) and is given by Equation 2.2.

$$
f_{\text {frpu }}=E_{\text {frp }} \epsilon_{\text {frpu }}
$$

Where,

$E_{f r p}=$ Elastic modulus of FRP.

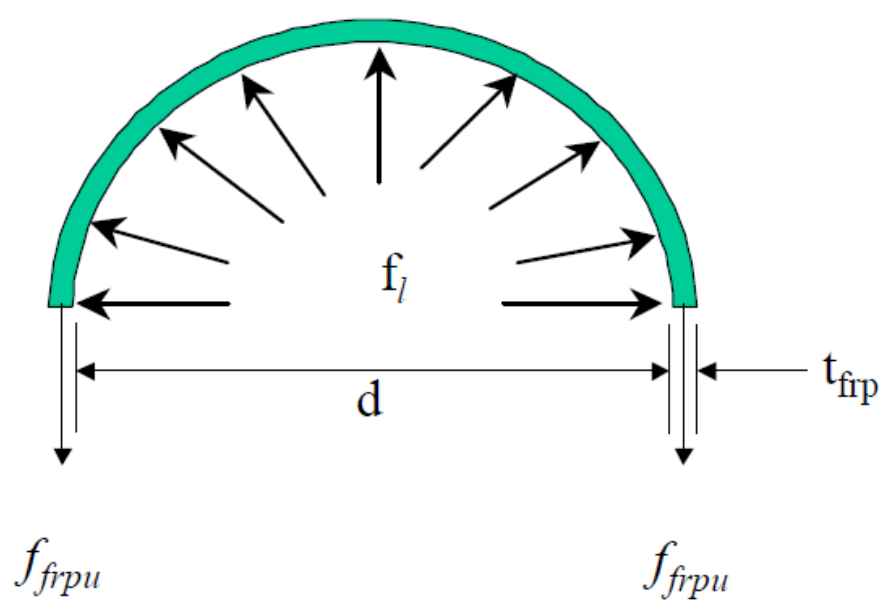

Figure 2.1: Schematic diagram of section of the confined concrete [7] 

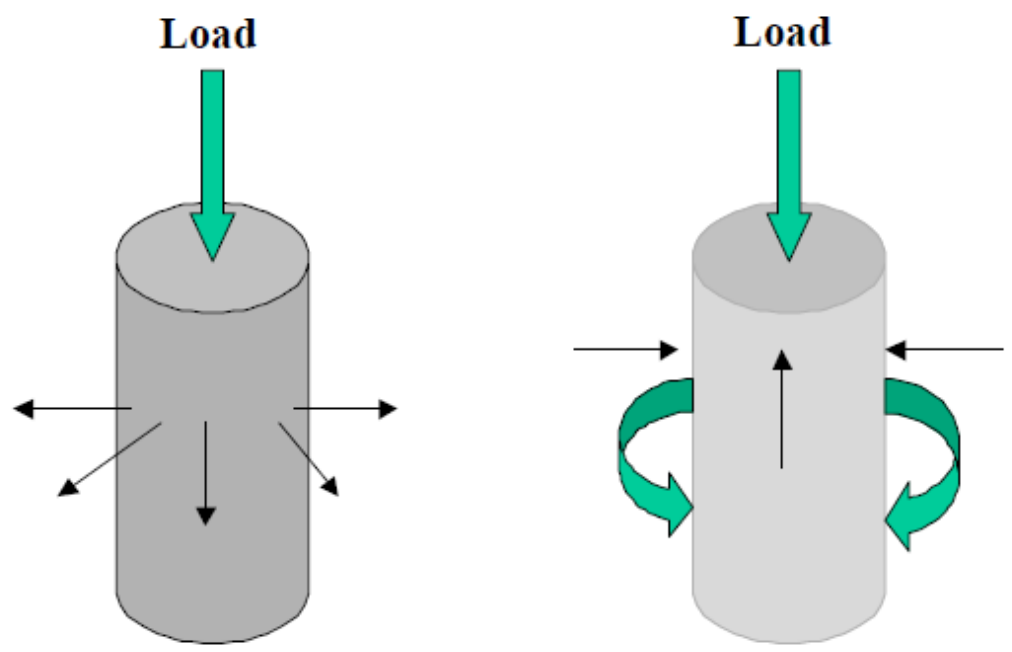

Figure 2.2: Lateral expansion (left), effect of confinement (right) [7]

\subsubsection{Underwater FRP Strengthening of Columns}

In the research carried out by Rajan Sen and Gray Mullins [3], two different FRP systems - pre-preg and a wet layup- for both glass and carbon were used successfully for repairing and rehabilitating corrosion-damaged partially submerged piles in the friendship trail bridge in Tampa bay. Further, on-site long term bond was evaluated after two years from the application of the wrap (Figure 2.3). Four piles in pier \#100 were wrapped with water-activated Aquawrap repair system, two with glass fabric and the other two with carbon fabric and two piles in pier \#101 were wrapped with wet layup Tyfo SHE-51A system. Of the two systems, bond test results were better for wet layup system than Aquawrap system. However, pre-preg water activated Aquawrap system was found to be easier to use. The wet layup system offered greater flexibility but did require on-site saturation of the resin, requiring greater preparation efforts. 


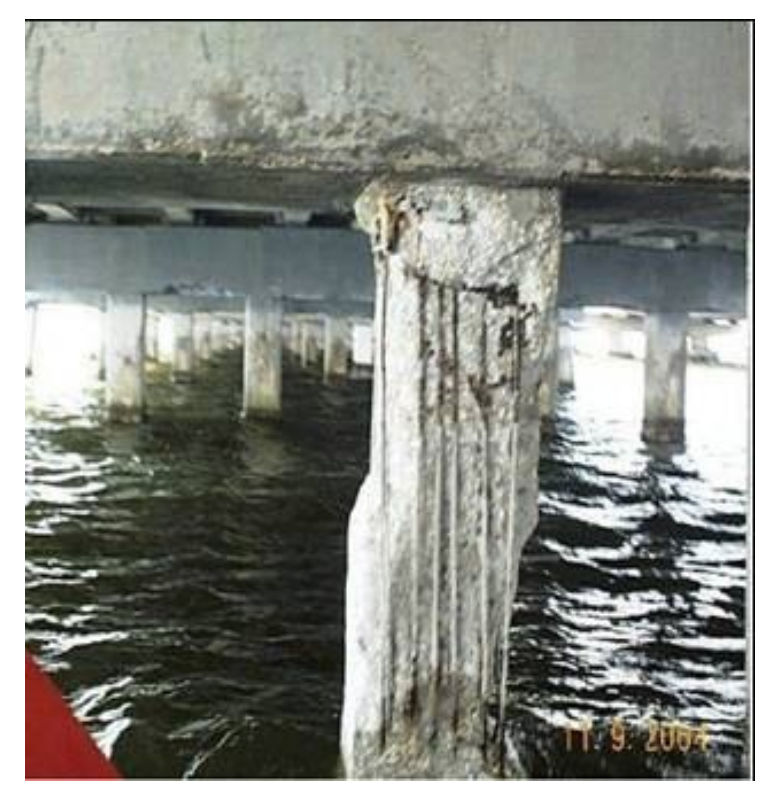

Figure 2.3: Damaged pile requiring repair [3]

Air Logistics Corporation [8] describes a successful installation of Aquawrap Repair System on two steel reinforced concrete pilings on a bridge over the North River located near Wilmington, NC. Concrete bridge pilings were subjected to splash zone damage due to abrasion or impacts by wave and tidal action, collisions with marine vessels and normal spalling. Traditionally, these repairs were done by variety of techniques such as: 1) concrete encapsulation using steel or composite forms, 2) wrapping with traditional composite systems, 3) simply patching the spalled concrete. This paper [8] highlights the difficulty and complications using concrete encapsulation (Figure 2.4), and conventional field saturated composite systems with epoxy requiring highly trained staff to perform the installation and also requiring the construction of coffer dam. This paper [8] highlights the ease of installation of Aquawrap system for the repair of concrete pilings without the use of coffer dams and suggests this as an economical solution for future repair and rehabilitation of underwater projects with minimum labor and very little environmental impact. 

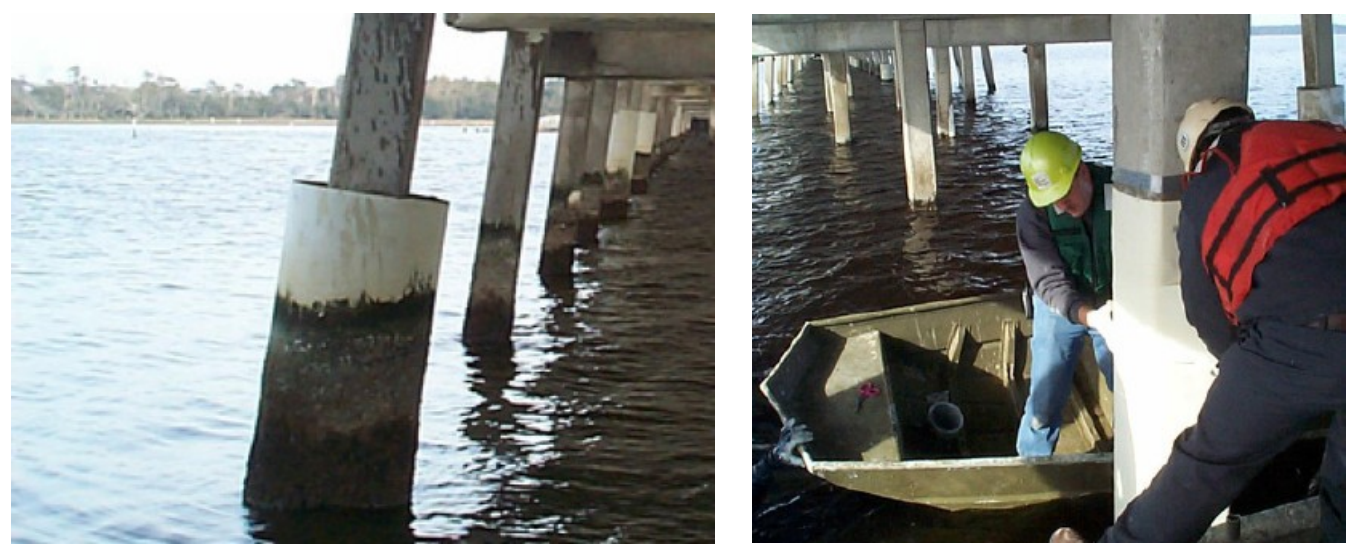

Figure 2.4: Concrete encapsulation (left) and Aquawrap installation (right) [8]

The research conducted by Gray Mullins [9] describes the field demonstration project where corroded pre-stressed piles in tidal waters were wrapped underwater with carbon and glass FRPs using unique water-activated urethane resin system without cofferdam construction. Linear polarization scheme was used to assess pre-wrap and post-wrap corrosion state. Total of four full-sized $35 \mathrm{~cm} \times 35$ $\mathrm{cm}$ square pre-stressed piles were wrapped, two with carbon and two with glass. Two other unwrapped piles served as controls, while two of these wrapped piles-one carbon and one glass- were instrumented to allow evaluation of their post-wrap performance. Instrumentation allowed determination of the corrosion potential over the unwrapped surface and the corrosion rate for the wrapped piles. It indicated that the corrosion rate in the wrapped specimens was consistently lower than those in its unwrapped counterpart. This paper [9] indicates that underwater wrapping is a viable system and highlights the prime importance on the surface preparation of concrete with removal of sharp corners. This paper also indicates that the underwater wrapping is very easy and less time consuming process and also suggests that the performance of the underwater wrapped piles is comparable to those wrapped inside a cofferdam based on corrosion monitoring data.

Aquawrap systems have been used in underwater as well as above water column repairs and have been performing well with increased structural capacity [10]. They have been used in repairing bridge columns in district \#6, NY and the installation was accomplished at low 
temperature environment $\left(\sim 50^{\circ} \mathrm{F}\right)[11]$. Aquawrap repair systems have also been used to repair mechanically damaged pipelines. Application of increased cyclic pressure on the pipelines resulted in $\sim 21$ times increase in fatigue life cycle [12]. Also from the study [13], the bonding between Aquawrap and concrete has been significantly increased by applying constant pressure around the wrapped area with a pressure bagging system during the curing process. The pressure bagging presses the FRP fabric and resin towards concrete surface not allowing them to fall down while working in vertical structures like columns and piers. In order to increase the bonding between Aquawrap fabric layers, high strength plastic film called Stricture Banding is used in order to compresses the composite layers impregnated with resin together during the curing process resulting better bonding between them [8].

The research by Chalid [14] discusses the performance of FRP under water especially debonding between FRP and surface of concrete or other materials when immersed in water. This paper verifies the performance of FRP materials with epoxy putty by performing several tests such as flexural strength test, bond test and chemical resistance test etc. It talks about the interfacial properties between FRP composites and polymeric matrix playing an important role in deciding final capacity of a composite material. This paper [14] says if interfacial shear strength of bonding material is rather low, the overall failure behavior of the retrofitted member would be affected mainly by low interfacial adhesive strength thus highlighting the necessity of high performance epoxy for underwater usage. This paper [14] says how such epoxy is obtained by adding epoxy silane coupling agent of silane system to base resin to increase bond strength with fibers and by adding dysfunctional reactive diluent to make it workable. In this research [14], three layers of FRP samples with size $300 \mathrm{~mm} \times 3 \mathrm{~mm} \times 50 \mathrm{~mm}$ were bonded with epoxy putty of thickness $2 \mathrm{~mm}$ and were tested in flexure and tension. In flexure, the stress-strain relationship came to be linear indicating FRP as brittle material. In tension, better 
tensile strength than that shown by other market products was achieved. Concrete beams of sizes 100 $m m \times 100 \mathrm{~mm} \times 500 \mathrm{~mm}$ were cast and 3 layers of FRP with epoxy putty in between FRP layers as well as between concrete and FRP were attached to the bottom side of the beam. Those beams were tested in flexure. Results showed significant strength increase and de-bonding failure was not the issue in this case. Thus, this paper [14] concludes that epoxy putty can be used for repair and strengthening of underwater structure using FRPs. The developed epoxy putty doesn't dissolve in water and has the same performance as the epoxies used in above ground constructions.

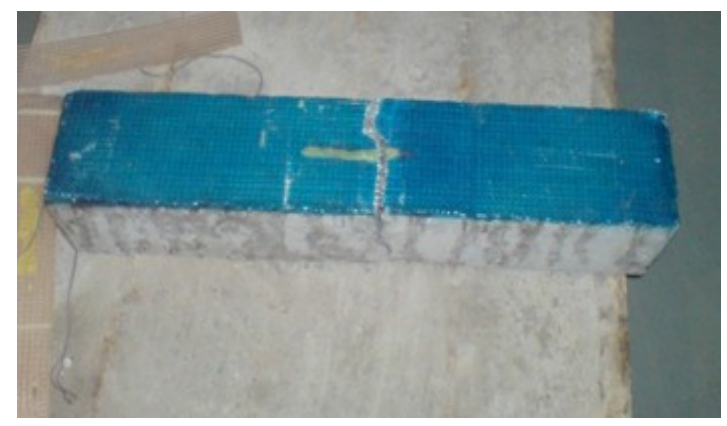

Figure 2.5: Flexural testing of FRP attached under the beam [14]

\subsection{Abrasion and Wear Mechanism}

Abrasion and wear is the natural phenomenon that occurs in almost all materials, from soft to the hardest, existing in the nature. Some materials wear down at a faster rate while some materials resist the abrasion and wear down at a slower rate. Wear is due to the interaction between two or more surfaces or materials in contact and depends on material properties along with the mechanical, physical, chemical, geometrical and topographical characteristics [15]. After the removal of wear material, formation of grooves, scratches, micro-chips and surface spots can be observed on the material surface.

In order to understand the wear, it is essential to know various underlying mechanisms that subsequently produce wear. Wear is typically distinguished as two body wear and three 
body wear. In two body wear, hard asperities or rigid grits pass over the material surface like a cutting tool where wearing down of the materials happens at a faster rate. Whereas, in three body wear, grits are not held rigidly and are free to roll between two bodies and wearing down of the material happens at a slower rate [16]. In two body abrasion, the wear down particles from the coating comes in contact between the roller and the coating surface and can influence the rate of wear of the material [16].

Typically, wear of a substrate material is a complex phenomenon that takes place due to various mechanisms like surface fatigue, abrasion, adhesion, and tribo-chemical reaction. Materials under contact, experience all these mechanisms which finally lead to the wear of a material. Surface fatigue is caused due to the alternating loads between base body and the counter body. Abrasion is the micro-cuttings due to repeated plowing and fracture of the base body due to hard interfaces of the counter body. Adhesion is the bond between two body layers that can detach the material of the base body and tribo-chemical reaction is the chemical reaction between counter body and the base body that can decrease the abrasion resistant properties of the base body [17]. The progress and extent of wear are dependent on the materials and finishes of the components, intermediate materials like oil, grease, water, contaminants, and surrounding influences like temperature, humidity, pressure and operating conditions. Various factors that influence the abrasive wear behavior are shown in Table 2-1.

Table 2-1: Factors influencing abrasive wear behavior [18]

\begin{tabular}{|c|c|}
\hline $\begin{array}{l}\text { Abrasive } \\
\text { properties }\end{array}$ & $\begin{array}{c}\text { Particle size, particle shape, hardness, yield strength, fracture properties, } \\
\text { concentration }\end{array}$ \\
\hline $\begin{array}{c}\text { Contact } \\
\text { conditions }\end{array}$ & $\begin{array}{l}\text { Force/ impact level, velocity, impact angle, sliding/rolling, temperature, } \\
\text { wet/dry, } \mathrm{pH}\end{array}$ \\
\hline $\begin{array}{l}\text { Wear material } \\
\text { properties }\end{array}$ & $\begin{array}{c}\text { Hardness, yield strength, elastic modulus, ductility, toughness, corrosion } \\
\text { resistance }\end{array}$ \\
\hline
\end{tabular}


Abrasive wear model is generally described by distinguishing four types of interactions between abrasive materials and a wearing material, namely micro-ploughing, micro-cutting, micro-fatigue, and micro-cracking [19]. Due to the rubbing action of abrasive particle on the wearing material, wearing materials in the line of contact is not ripped off but displaced sideways to form ridges. After repeated micro-ploughing action of abrasive particles, wearing material is seen to lose some volume [20]. Materials may also be ploughed aside repeatedly by particles and may break off by fatigue, i.e. micro-fatigue. Micro-cutting results in the complete volume loss by chipping of materials from the wear grooves and micro-cracking occurs when the concentrated stresses on abrasive particles are high where large debris is detached from the wearing surface owing to crack and propagation. Micro-cracking is an important phenomenon on brittle materials while micro-ploughing and micro-cutting in ductile materials [19], [20].

\subsubsection{Wear Resistance and hardness of the Materials}

Hardness of a material is defined as the resistance to penetration by an indenter. And we can say that hardness of the wearing material affects the penetration depth of abrasive particles during abrasion but fails in predicting abrasive wear resistance alone. Hardness of metals can be increased substantially by cold working but the abrasive wear resistance is not increased accordingly [21]. Hardness fails in predicting wear resistance as it cannot characterize the interactions between abrasive particles and the wearing materials [21]. For brittle materials, wear resistance is seen to be improved by increasing the fracture toughness. Nevertheless, hardness and fracture toughness alone cannot describe the abrasive wear resistance. FRP composites are good against brittle fracture as they have high hardness and high fracture toughness. Abrasive wear resistance in composites depends upon the volume fraction and distribution of embedded fibers, the properties of matrix and the bonding between the matrix and fibers. Hard abrasive 
particles can cut or crack larger fibers while they can easily remove smaller chopped fibers. Soft abrasive particles can dig out smaller fibers while the larger ones provide better resistance. Larger fibers not properly bonded to the matrix can easily be pulled but properly bonded ones can crack or blunt softer abrasive particles [21].

\subsubsection{Surface Wettability on Friction and Wear}

According to the research [22], it shows that the surface wettability of the material has an effect in tribological tests. Depending upon different surface wettability, different distribution of the drops of water is seen on the material. In the test, where both pin and substrate surfaces are hydrophilic, the layer of water in between surfaces is seen very thin due to same affinity for water from both materials and this thin layer does not make a layer capable of hydrostatic lift but still lubricates as an adhesive layer and prevents seizing on the material surface and also water washes the debris which reduces further wear down. In another test with pin being hydrophilic and substrate being hydrophobic, a continuous layer of water is seen between the pin and substrate with good lubrication [22]. The hydrophobic surface of the substrate against the hydrophilic surface of the pin promotes the formation of a layer of water which has hydrostatic lift that reduces contact between the sliding surfaces. In test with hydrophobic pin and hydrophilic substrate, repulsion of water from the small surface of the pin cannot produce a lifting layer of water but still helps in lubricating. When the surfaces of pin and substrate are both hydrophobic, it has been noted that there is no formation of any layer of water between the surfaces with no lubricating effect [22]. In this research, the abrasion tests are performed in different available abrasion resistant coatings with a steel ball as an abrader which is a good hydrophilic material in Taber reciprocating abrader and the abrasion results have been correlated with coating materials surface wettability. 


\subsubsection{Coating Materials}

As materials used in infrastructure application undergo erosion, abrasion, corrosion, and wear, it is necessary to protect those materials. Coating of those materials with better abrasion and corrosion resistant materials improves the surface hardness and abrasion resistance. Many coatings with inorganic materials like silicon, aluminum, titanium, and zirconium metal alkoxides as well as organic materials like siloxanes, polyamides, ketones have been processed to form hybrid composite coatings of superior hardness. In addition to these materials, ceramics have been particularly used for obtaining corrosion and wear resistant coatings [23]. Coating materials themselves wear down when in contact with foreign materials, thus protecting the substrate material. In this research, different commercially available coatings have been evaluated in their abrasion resistance capacity.

\subsubsection{Ceramic Coatings}

In this research, ceramic based composite coatings have been used to provide wear resistance. Ceramics are inorganic, non-metallic materials made from compounds of a metal and a nonmetal. Metal oxides (A12O3, FeO, etc.) are common examples of ceramics, but other compounds such as carbides and nitrides are also included. Porcelain, glass, bricks and refractory materials are some examples of traditional ceramics. Now, due to advances in material science ceramics materials tough enough to withstand engine environments have been produced. Ceramics are used in a variety of applications including window glass, implantable teeth, brick, ceramic bones, nuclear fuel, tennis racquets, solid-state electronic devices, engine components, cutting tools, valves, bearings, and chemical-processing equipment [24]. Metal oxides are also very hard and are very resistant to chemical attack so they can be an ideal solution when wear and corrosion are both present [25]. Ceramics have wide range of properties based on their type 
which is shown in Table 2-2. They are generally more brittle than metals and can have similar stiffness (modulus of elasticity) and similar strength, particularly in compression. But in a tensile test they are likely to fail at a much lower applied stress because the surfaces of ceramics always contain minute cracks ("Griffith cracks"), which magnify the applied stress [24]. Ceramics are most often produced by compacting powders into a body which is then sintered at high temperatures. During sintering, the body shrinks and the grains bond together producing a solid material [24].

Table 2-2: Different types of ceramics [24]

\begin{tabular}{|l|l|}
\hline \multicolumn{1}{|c|}{ Ceramic Type } & \multicolumn{1}{c|}{ Characteristics } \\
\hline \multicolumn{1}{|c|}{ Alumina } & $\begin{array}{l}\text { Abundant and easily fabricated. } \\
\text { Good strength and hardness. } \\
\text { Wear and temperature resistant. } \\
\text { Good electrical insulators. } \\
\text { Low dielectric loss. }\end{array}$ \\
\hline Beryllium Oxides & Exceptionally high thermal conductivities \\
\hline Zirconia & $\begin{array}{l}\text { Extreme inertness to most metals. } \\
\text { Good toughness and strength. }\end{array}$ \\
\hline \multicolumn{3}{|c|}{ Glass Ceramics } \\
\hline Glass-Ceramics & $\begin{array}{l}\text { Low, medium or high thermal expansion depending on composition. } \\
\text { Good electrical insulators. } \\
\text { Transparent } \\
\text { Can be machined with steel tools. }\end{array}$ \\
\hline Silicon Nitrides & $\begin{array}{l}\text { Resistant to high temperatures, thermal stress and shock. } \\
\text { High strength and oxidation resistant. } \\
\text { Good electrical insulators. }\end{array}$ \\
\hline Boron Carbide & $\begin{array}{l}\text { High hardness and low density. } \\
\text { Best abrasion resistance of any ceramic. } \\
\text { Low strength at high temperatures. }\end{array}$ \\
\hline Silicon Carbides & $\begin{array}{l}\text { Low electrical resistivity. } \\
\text { High strength and resistance to chemical attack. } \\
\text { High temperature and thermal stress. }\end{array}$ \\
\hline Tungsten Carbides & $\begin{array}{l}\text { Used for tool tips. } \\
\text { Excellent hardness and mechanical strength. } \\
\text { Good thermal conductivity. } \\
\text { Good wear and abrasion resistance. }\end{array}$ \\
\hline
\end{tabular}




\begin{tabular}{|c|c|}
\hline \multicolumn{2}{|r|}{ Glass } \\
\hline $\begin{array}{l}\text { Oxide (silica) } \\
\text { Silicates } \\
\text { Phosphates } \\
\text { Borosilicates }\end{array}$ & $\begin{array}{l}\text { Good resistance to thermal shock. } \\
\text { Large range of special optical characteristics. } \\
\text { Transparent. } \\
\text { Low thermal expansion and high dielectric strength. } \\
\text { Good chemical resistance. }\end{array}$ \\
\hline \multicolumn{2}{|r|}{ Carbon and Graphite } \\
\hline Carbons and Graphites & $\begin{array}{l}\text { Poor strength except when produced as fiber. } \\
\text { Good electrical and thermal conductivity } \\
\text { Creep resistant at high temperatures in non-oxidizing conditions. } \\
\text { Self-lubricating. } \\
\text { Good refractoriness and thermal shock resistance. } \\
\text { Low density and chemically inert. }\end{array}$ \\
\hline $\begin{array}{l}\text { Carbon/ } \\
\text { Carbon Composites }\end{array}$ & $\begin{array}{l}\text { High strength and low coefficient of thermal expansion. } \\
\text { Excellent thermal shock resistance. } \\
\text { Superior toughness, excellent thermal and electrical conductivity. } \\
\text { Resistance to corrosion and abrasion. }\end{array}$ \\
\hline \multicolumn{2}{|r|}{ Porcelain } \\
\hline Porcelain & $\begin{array}{l}\text { Good chemical and thermal resistance. } \\
\text { High density, strength, resistivity and dielectric strength } \\
\text { Good thermal shock, wear and hot strength. } \\
\text { Chemically inert. }\end{array}$ \\
\hline \multicolumn{2}{|r|}{ Ceramic Fibers } \\
\hline Fibers & $\begin{array}{l}\text { Oxides spun to fiber and bulked to felt. } \\
\text { Used for high temperature insulation }\end{array}$ \\
\hline
\end{tabular}

\subsection{Conclusions}

Underwater wrapping with Aquawrap system (pre-preg and water activated urethane resin) is undoubtedly an easier, fastest and cost-effective to rehabilitate submerged columns and piers. The field implementation of Aquawrap has been successful in many projects done by different researchers. The strength, stiffness and ductility increment due to Aquawrap repair is investigated in this research. The bonding between the Aquawrap and the concrete is reported by Rajan Sen to be requiring further improvement though confinement is contact-critical. However, good bonding is required between the wrap and the substrate. Long term performance data of underwater wrapped concrete structures are still to be understood. This study deals with the 
strength, stiffness and ductility increment due to underwater wrapping and also successfully presents the field implementation of underwater wrapping in two major sites.

Developing an effective abrasion and corrosion resistant coating for metallic and nonmetallic surfaces has always been a challenge to researchers and experts. Wear is a complex phenomenon which occurs due to the interaction between two or more surfaces or materials in contact and these interactions depend on material properties along with their mechanical, physical, chemical, geometrical and topographical characteristics. Different factors like the particle size, particle shape, contact conditions, $\mathrm{pH}$, temperature, moisture, force/impact level, hardness of the wear material, toughness, elastic modulus, yield strength etc. play an important role in the wear mechanism. This study deals with several coating systems and their wear characteristics. 


\section{CHAPTER 3 UNDERWATER WRAPPING}

\subsection{Introduction}

Glass and carbon FRPs (Fiber Reinforced Polymers) are being increasingly employed for new construction and repair/rehabilitation of in-service civil and military infrastructure systems. FRPs offer the advantages of high strength to weight ratio, confinement effects to enhance strength and ductility of underlying substrate, excellent durability, and also resistance against corrosion, moisture absorption and chemical attack. For example, FRP wraps act as protective barriers around a concrete substrate and inhibit the flow of moisture and air into concrete members, thus reducing the rate of corrosion of steel reinforcement. Bonding of wraps to structural members is similar to wall paper application and the process is referred to as wetlayup. Alternately, FRP wraps may be pre-saturated or pre-impregnated with resins and are referred to as pre-pregs.

Under this US Army Corps of Engineers (USACE) sponsored research, concrete cylinders were wrapped underwater at WVU-CFC lab facilities to evaluate their improved load capacity, stiffness, and ductility due to confinement effects (Figure 3.1). FRP wraps also act as protective membranes and inhibit the flow of moisture and air into concrete members, thus reducing the rate of corrosion of steel reinforcement.

Generally, repair or retrofit of concrete structures with FRP wraps is done in the absence of water. For underwater wrapping, coffer dams are constructed around the concrete substructures and FRP wrapping is carried out such that primer and resin curing is facilitated under dry conditions by creating water-free zones. Due to the advancement in the field of science, adhesives and resins that can cure under water immersion are commercially available. This study 
has evaluated the structural response of wrapped concrete structures using specialty primer, resin, and GFRP system developed for underwater wrapping of concrete structures.

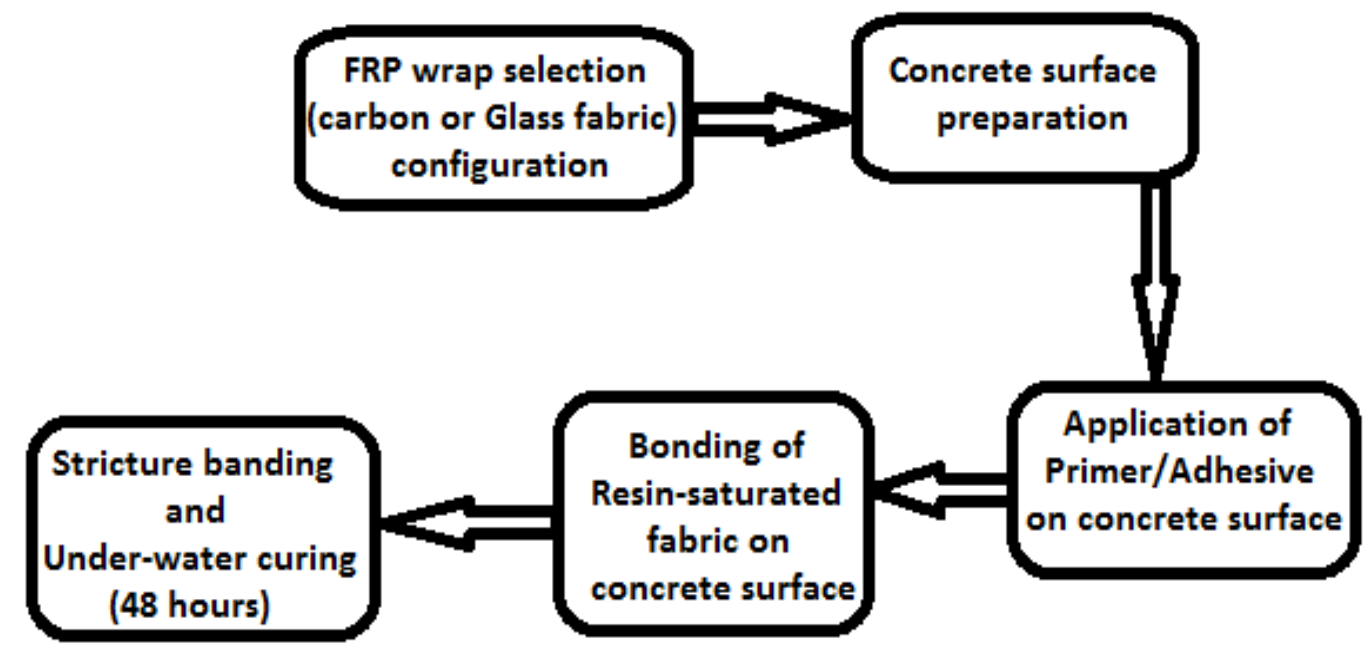

Figure 3.1: Simplified underwater wrapping steps

In this research, cylinders were cast with two types of materials i) low strength concrete representing as a pier-like concrete section of discharge ports in lock and dam system, and ii) underwater high early strength and rapid set structural mortar (representing a repair material for underwater discharge ports.

\subsection{Materials and Properties}

\subsubsection{Concrete}

First batch of low-strength concrete $(\sim 2.5 \mathrm{ksi})$ was ordered from a local concrete plant and thirty-six (36) plain concrete cylinders (4" x 8") were prepared.

Second batch of 12 concrete cylinders (4" x 8") were prepared with "five star structural concrete" i.e. underwater rapid set and high early strength structural mortar. 

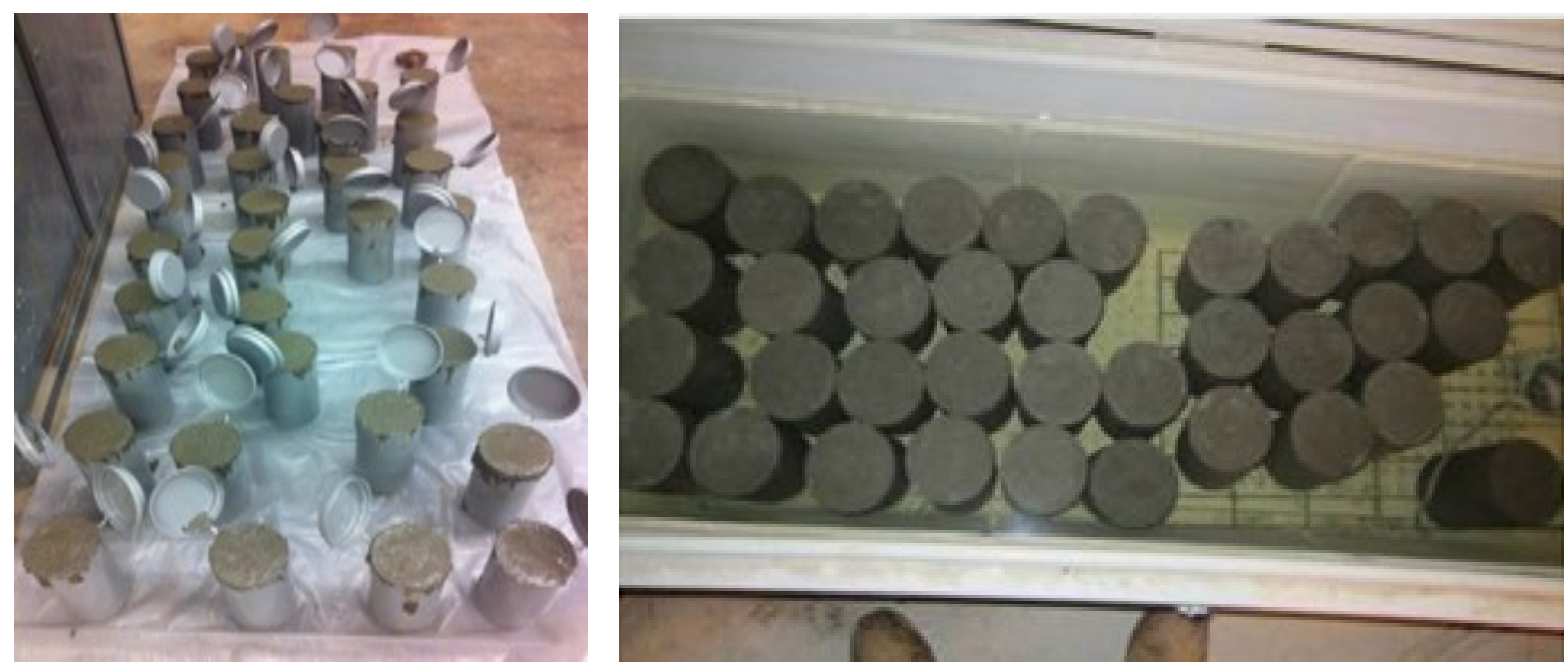

Figure 3.2: Casting of concrete (left) and curing of concrete (right)

\subsubsection{Aquawrap Fabric}

It is a pre-preg system, where fabric is pre-impregnated with water-activated urethane resin (Figure 3.3). The advantages of Aquawrap include: low cost, ease of use, ability to cure in wet and underwater conditions, customizable dimensions, heat resistance up to $1420 \mathrm{C}$ (288 0F), and others [10]. When water cured, Aquawrap is impervious to fuels, most chemicals and solvents (acetone, MEK, toluene, gasoline, ethyl alcohol and others) and provides high strength \& durability. It is odorless, non-flammable, and bonds permanently to a wide variety of surfaces such as metals, composites, concrete, plastics, and wood. Aquawrap is hermetically sealed in foil pouches and opened just before the application of FRP to prevent premature curing by atmospheric moisture. Some of the manufacturer listed properties of Aquawrap are listed in Table 3-1. 


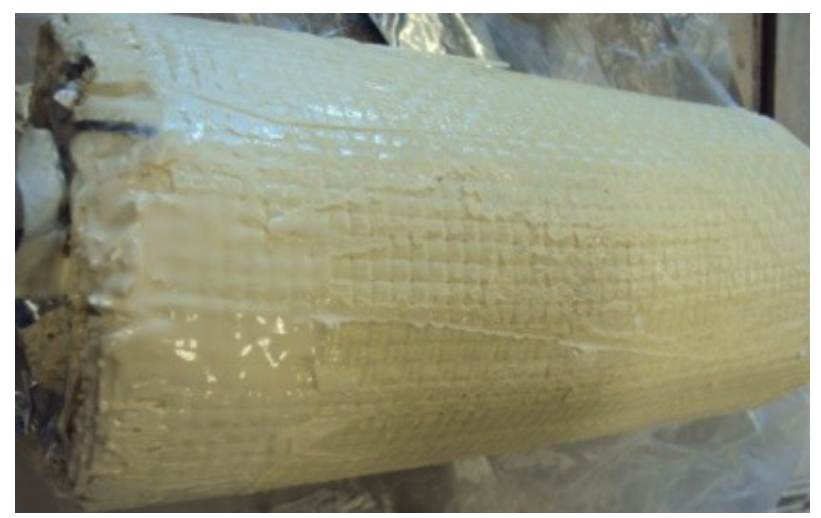

Figure 3.3: Aquawrap with pre-saturated resin

Table 3-1: Properties of Aquawrap [10]

\begin{tabular}{|l|l|l|}
\hline Properties & G-03 Fabric & G-05 Fabric \\
\hline Reinforcement & $\begin{array}{l}\text { Woven glass, } \\
\text { bi-axial fabric }\end{array}$ & $\begin{array}{l}\text { Woven glass, } \\
\text { bi-axial fabric }\end{array}$ \\
\hline Tensile strength & $60,900 \mathrm{psi}$ & $45,400 \mathrm{psi}$ \\
\hline Tensile modulus & $3.02 \mathrm{msi}$ & $2.28 \mathrm{msi}$ \\
\hline Tensile load per ply & $956 \mathrm{lbs} /$ in. width & $1299 \mathrm{lbs} /$ in. width \\
\hline Thickness & $16 \mathrm{mils}$ & $28.5 \mathrm{mils}$ \\
\hline Working time & \multicolumn{2}{|c|}{$30-40$ minutes } \\
\hline Application temperature & \multicolumn{2}{|c|}{$40-200^{0} \mathrm{~F}$} \\
\hline Service temperature & \multicolumn{2}{|c|}{$-40-250^{\circ} \mathrm{F}$} \\
\hline Cure time(dry to touch) & \multicolumn{2}{|c|}{60 minutes } \\
\hline Full cure & \multicolumn{2}{|c|}{2 days $\left(77^{0} \mathrm{~F}\right)$} \\
\hline
\end{tabular}

\subsubsection{BP-4 Primer /Adhesive}

Application of primer promotes bonding between FRP and the substrate, and functions as a corrosion barrier. For underwater application, grey colored primer "BP-4" (Figure 3.4) is used. It is a $100 \%$ solid two-component based epoxy system with the properties shown in Table 2 . It is solvent free and consists of microfibers to resist hairline cracking and chipping [26]. It has 
excellent chemical and abrasion resistance, and bonds to concrete (wet or dry), fiberglass, \& wood surfaces. When working under water, BP-4 adhesive system can also be used as a final UV protective coating over a cured Aquawrap installation [26].

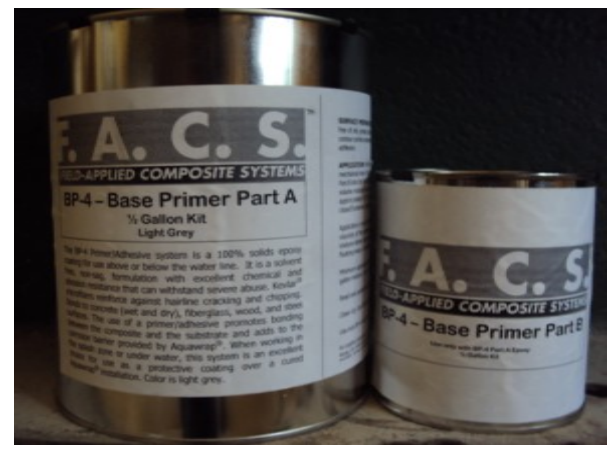

Figure 3.4: Base primer 4 (BP-4)

Table 3-2: Properties of base primer/adhesive (BP-4) [26]

\begin{tabular}{|l|l|}
\hline Compressive Strength & $68.9 \mathrm{MPa}(10,000 \mathrm{psi})$ \\
\hline Tensile Strength & $33.1 \mathrm{MPa}(4,800 \mathrm{psi})$ \\
\hline Flexural Strength & $45.5 \mathrm{MPa} 6,600 \mathrm{psi}$ \\
\hline Application Temperature & $\left(13-49^{0} \mathrm{C}\right)\left(55-120^{0} \mathrm{~F}\right)$ \\
\hline Full cure & 7 days @ $25^{\circ} \mathrm{C}\left(77^{0} \mathrm{~F}\right)$ \\
\hline
\end{tabular}

\subsubsection{Stricture Banding}

It is used to compress and consolidate the underwater-installed Aquawrap composite system. Using Stricture banding is a critical step in ensuring proper bonding of the wrap to concrete substrate (Figure 3.5).

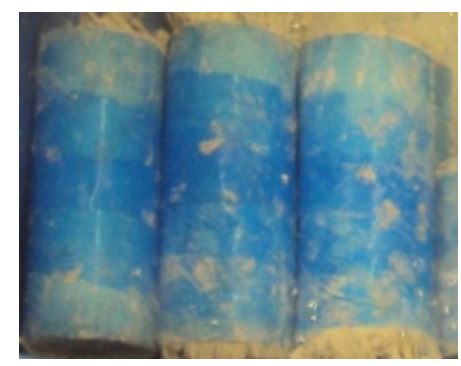

Figure 3.5: Stricture banding on concrete cylinders 


\subsection{Wrapping Details}

After 28 days of curing, concrete cylinders were wrapped with Aquawrap GFRP fabrics. The wrapping steps and procedures are described in following sections. However, wrapping procedure applicable to underwater field conditions are provided in Appendix-A.

\subsubsection{Surface Preparation}

The surface of the concrete cylinder was cleaned with wire brush all around, wiped clean or air-blown (Figure 3.6). Cleaning can also be done with a low speed grinder using low abrasion resistant sand paper or disc. Depending on the area and required surface texture, coarser 220 grit to finer 1000 grit sand paper or disc is used. The purpose of cleaning the surface is to make sure it doesn't have any protrusions or large cavities or loose scales where stress concentrations can be developed or bond may be compromised. For underwater purposes, pressurized water jet cleaning method or powered hand tools operated at up to $4500 \mathrm{rpm}$ with rotary cleaning attachments (steel brushes or abrasive 80-120 grit sanding discs, e.g. Stanley hydraulic grinder) can be used along with suitable degreasing agents to clean the surface of concrete to make it free from oil/grease, dirt, loose materials, calcareous debris, and spalling concrete. Large cavities are filled with grout to minimize stress concentrations. Any filler material used to contour surface anomalies must be sanded and abraded to remove protrusions and provide good adhesion with even surface texture. 

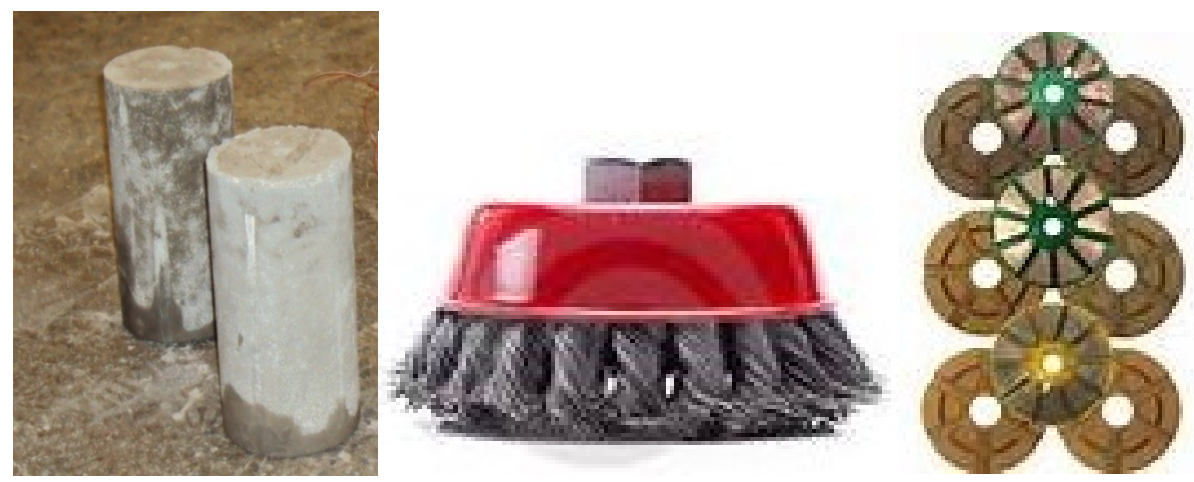

Figure 3.6: Concrete cylinder, steel disc brush, concrete grinding discs

\subsubsection{Application of Base Primer\#4 (BP-4) Adhesive/Primer}

Following surface preparation, BP-4 Primer part B and BP-4 Primer part A are mixed in the manufacturer recommended proportion with a spatula. Pot life of the mix is approximately 55-75 minutes at $75^{0} \mathrm{~F}$. Recommended application temperature ranges from a minimum of $55^{0} \mathrm{~F}$ to a maximum of $120^{\circ} \mathrm{F}$ (Table 3-2: Properties of base primer/adhesive (BP-4) ). The mixed primer is applied all around the concrete surface under water (Figure 3.7). The heavily viscous primer mixture allows the product to be easily applied underwater without floating away from the concrete surface. A spreader can be used for large surface applications while hand gloves can be employed for smaller surface area of the cylinder. The minimum recommended application thickness is around 40 mils.
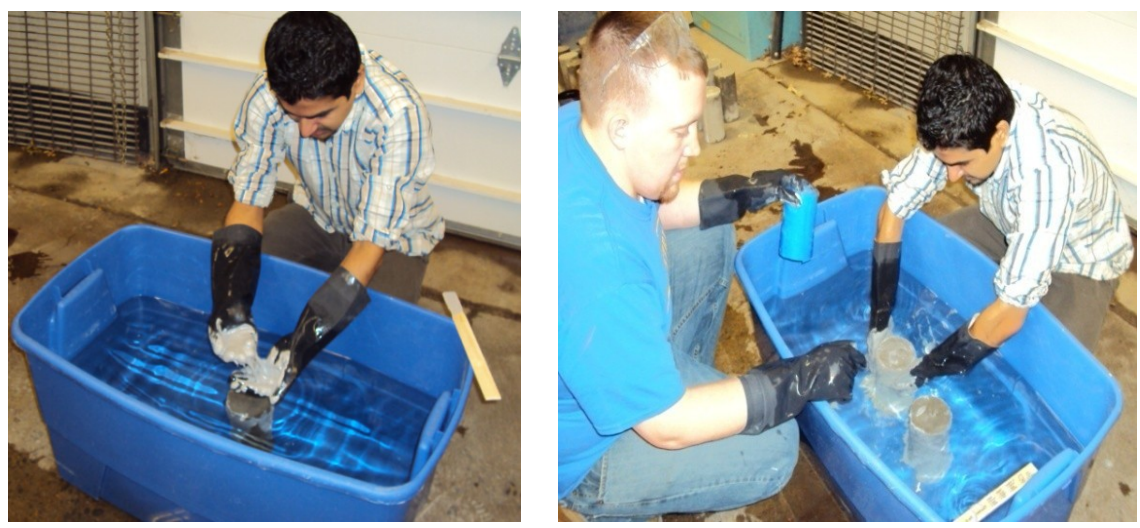

Figure 3.7: Underwater application of BP-4 primer (left) and Aquawrap on concrete cylinder (right) 


\subsubsection{Installation of Aquawrap around the Concrete Cylinder}

After the underwater application of BP-4 Primer on the concrete cylinder's surface, the Aquawrap pouch is opened. Once the Aquawrap is exposed to the humidity in the air, it begins to cure with gel time of 60 minutes. Hence, work details should be planned prior to opening the bag. Depending upon the number of layers, required length of the wrap is calculated and cut. Aquawrap is tightly hand wrapped around the concrete cylinder creating certain amount of tension in the fabric (Figure 3.8).
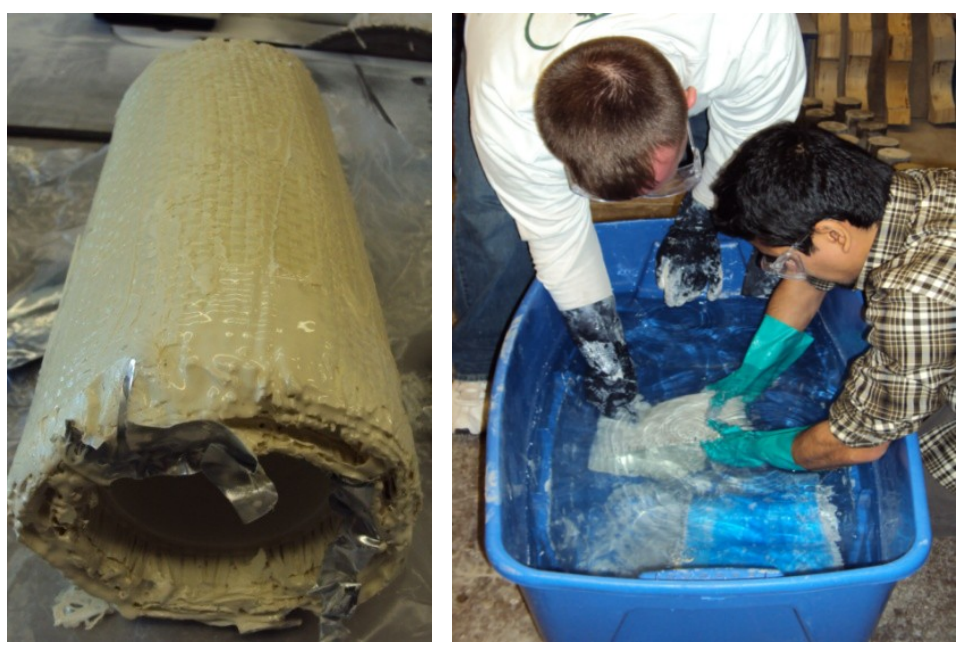

Figure 3.8: Aquawrap opened from the pouch (left) and underwater wrapping of cylinders (right)

\subsubsection{Stricture Banding and Curing}

After completion of wrapping around the concrete cylinder, Aquawrap is trimmed and is integrated into the preceding layer by applying Stricture Banding around the wrapped cylinder. The first layer of Stricture Banding has to be smoothly applied with only slight tension while subsequent layers should be tightly stretched in the same direction of FRP wrapping. Surface of the Stricture banding is perforated using perforator in order to create channels for the release of gaseous bubbles released during curing (Figure 3.9). When the wrapped cylinders are cured for 
about 1-2 hours, the stricture banding is removed and the cylinders are left to cure fully which takes about 2 days under water at $77^{0} \mathrm{~F}$.

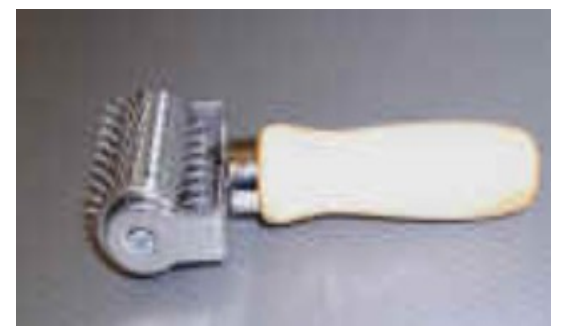

Figure 3.9: Perforator tool
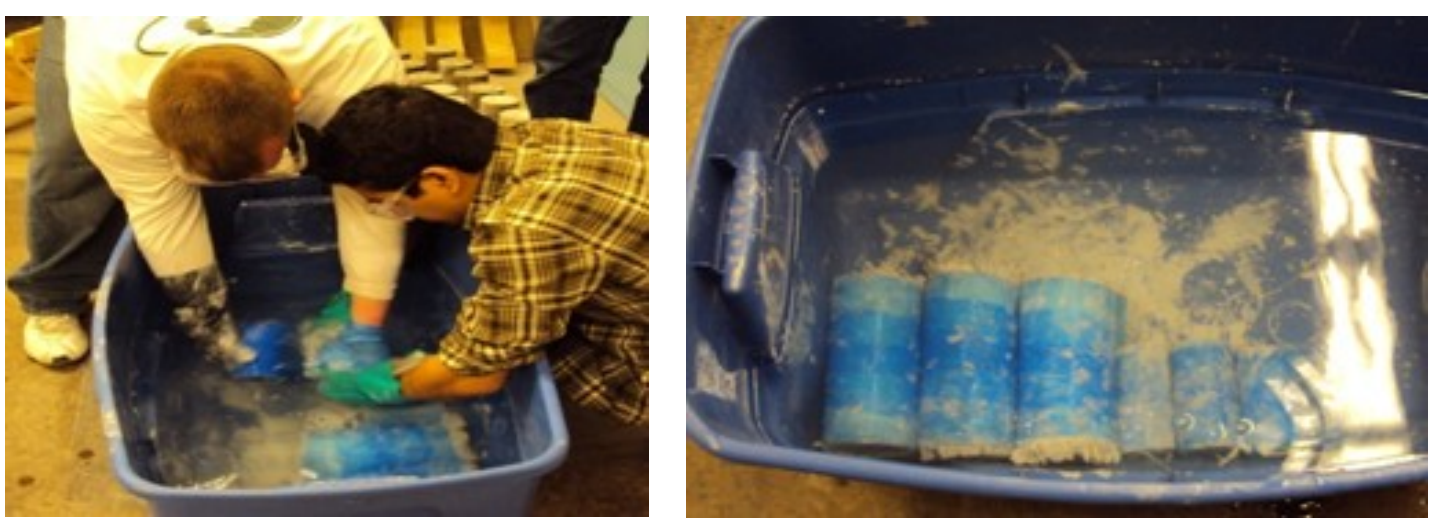

Figure 3.10: Stretching Stricture banding (left) and Curing of wrapped cylinders (right)

\subsection{Experimental Methods on Cylinders \& Wraps}

Axial Compression tests were conducted on non-wrapped and wrapped concrete cylinders to evaluate their strength, stiffness, and ductility improvements. Concrete cylinders were prepared in accordance with ASTM C-39. Also, underwater curable Aquawrap GFRP fabrics were separately tested as strips to evaluate their strength and stiffness properties. 


\subsubsection{Axial Compression Test}

Axial Compression tests were conducted on non-wrapped and wrapped concrete cylinders using an Instron 1000HDX (1,000 KN or 224 kips) universal hydraulic testing system (Fig. 6) to evaluate their strength, stiffness, and ductility improvements. Concrete cylinders were prepared in accordance with ASTM C-39 and were placed at the center of the lower mounting table of the Instron 1000HDX for testing. Cylinders were capped with steel capping plates on both ends with internal stiff elastomeric pads that help achieve uniform and concentric axial loading. Cylinders were loaded as per ASTM C39 specified rate, such that failure occurred between 2-5 minutes.

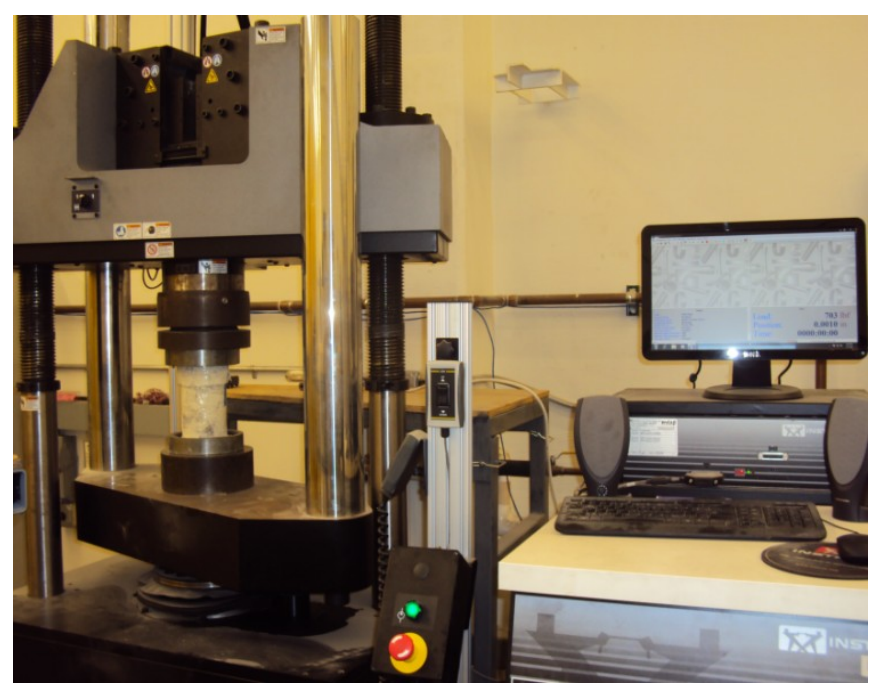

Figure 3.11: Axial compression test of wrapped cylinder on Instron 1000HDX

\subsubsection{Tension Test on FRP Strip Specimens}

Underwater curable Aquawrap GFRP fabrics were separately tested as strips to evaluate their tensile strength and stiffness properties. Aquawrap strip specimens with 1, 2, 3, and 5 layers of fabrics were prepared by stacking wraps one over another underwater applying compression on top. Those laminates with different number of fabrics were cured underwater for at least 48 
hours and cut to a dimension of $250 \mathrm{~mm} \times 25.4 \mathrm{~mm}$ (12"X 1"). The strip specimens were tested in tension using the Instron $8501(100 \mathrm{KN}$ or $22 \mathrm{kips})$ (Figure 3.12).

Test strips were bonded with strain gage at mid-height in the axial direction and an extensiometer was used to verify strains (Figure 3.12). To avoid potential failure in the grip region of Aquawrap strips with both lower (thinner) and higher (thicker) number of layers, steel plate tabs were attached at both ends of the specimen. Aquawrap strips were tested in a strain controlled manner to failure as per ASTM D3039 and ASTM D638.

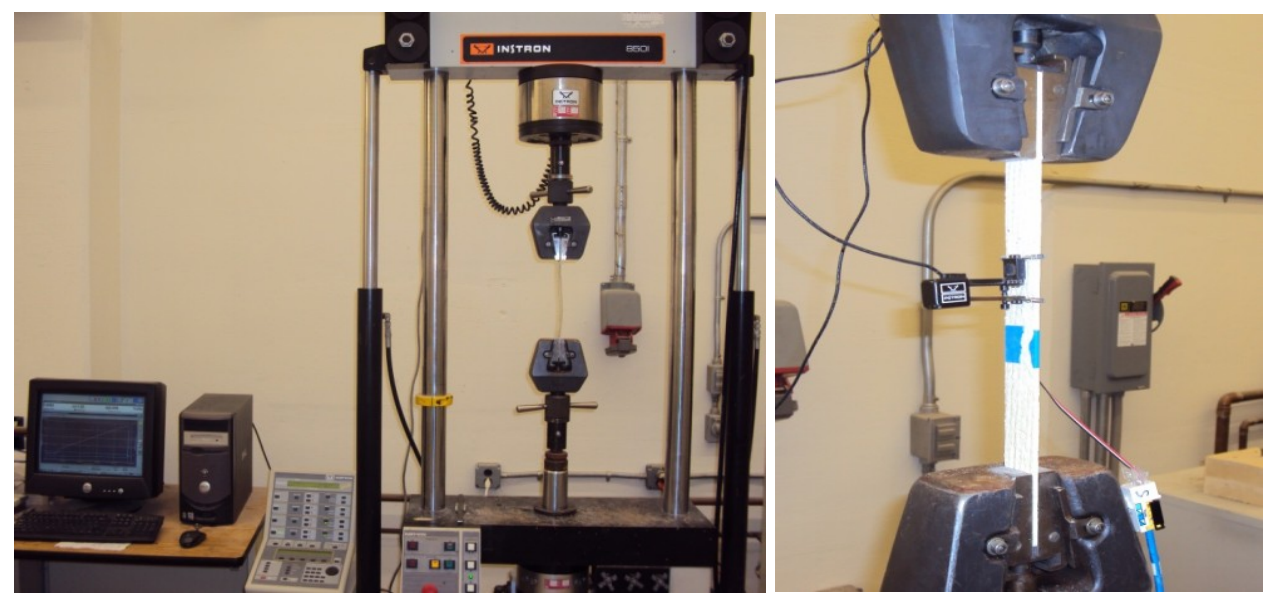

Figure 3.12: Testing of Aquawrap strip on Instron 8501 (left) \& test strip attached with extensiometer and strain gage (right)

\subsection{Results and Discussions}

The results and discussions are provided in the following sub headings (3.5.1 to 3.5.5).

\subsubsection{FRP Strip Specimens}

Aquawrap (G-05) FRP strip specimens with 1,2, 3 and 5 layers of wrap were tested under tension and the results are summarized in Table 3. Each layer of Aquawrap was $0.72 \mathrm{~mm}$ 
(0.0285") in thickness. A typical tensile stress-strain diagram for single layer GFRP Aquawrap strip specimen is shown in Figure 3.13.

Table 3-3: Axial tensile strength of $304.8 \mathrm{~mm}$ X $25.4 \mathrm{~mm}$ (12" X 1") Aquawrap strips

\begin{tabular}{|c|c|c|}
\hline No. of FRP layers & $\begin{array}{c}\text { Average load } \\
\mathrm{kN}(\mathrm{lbf})\end{array}$ & $\begin{array}{c}\text { Average stress } \\
\mathrm{MPa}(\mathrm{psi})\end{array}$ \\
\hline 1 & $5.0(1121)$ & $271(39,333)$ \\
\hline 2 & $10.3(2323)$ & $281(40,754)$ \\
\hline 3 & $16.7(3756)$ & $303(43,930)$ \\
\hline 5 & $25.0(5615)$ & $272(39,404)$ \\
\hline
\end{tabular}

Note: While cutting bi-directional Aquawrap fabric for a width of $25.4 \mathrm{~mm}$, number of fibers cut might vary and influence the maximum load to failure.

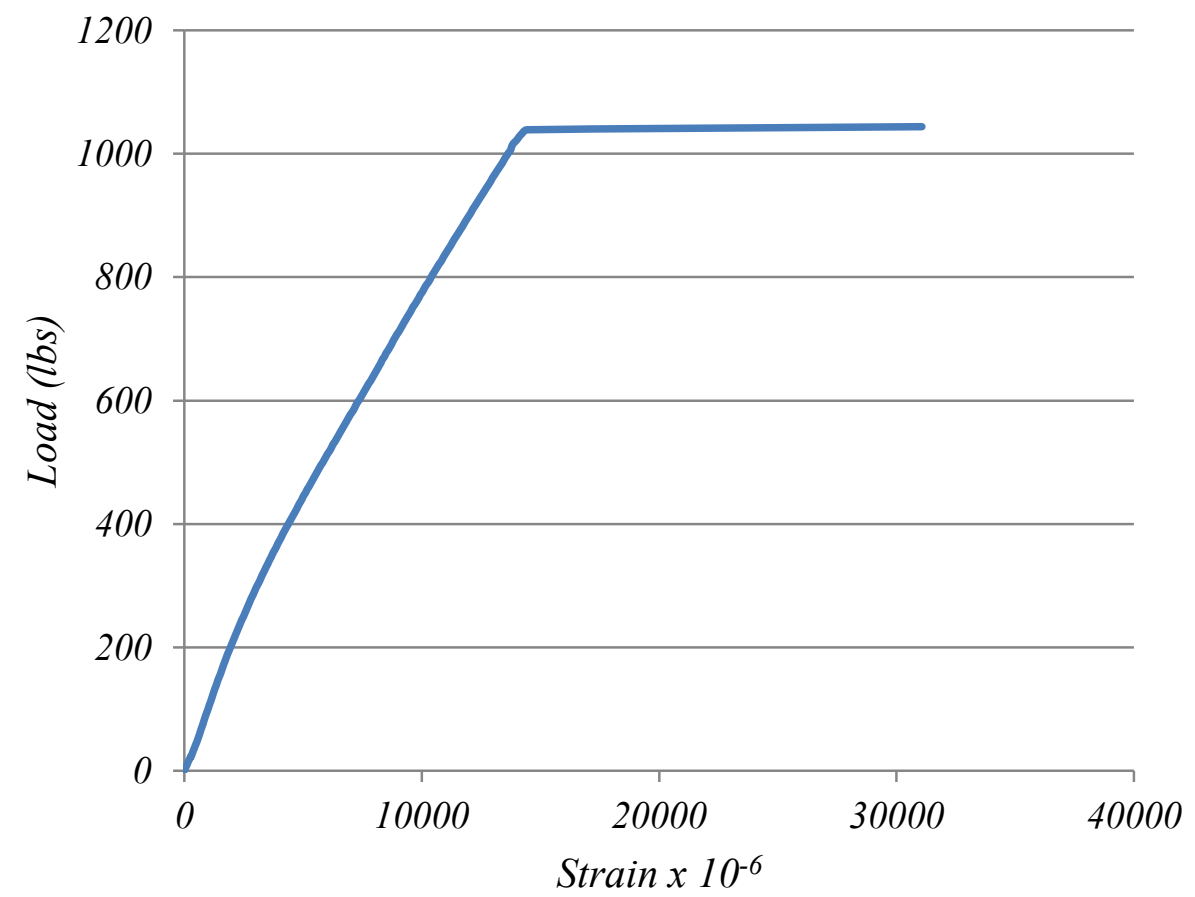

Figure 3.13: Load vs. Strain plots for 12"X1" single layer strip 


\subsubsection{Concrete Cylinders}

Compression tests were performed on $100 \mathrm{~mm}$ x $200 \mathrm{~mm}$ (4” x 8”) non-wrapped lowstrength $\sim 13.8 \mathrm{MPa}(2 \mathrm{Ksi}$ ) concrete cylinders as well on underwater wrapped concrete cylinders. Minimum of three concrete cylinders were wrapped with 1,2, 3 and 5 layers of Aquawrap G-05 FRP fabric. The maximum axial compressive loads to failure are shown in Table 3-4.

Table 3-4: Strength of non-wrapped/wrapped concrete cylinders \& their \% increase in strength

\begin{tabular}{|c|c|c|c|c|c|c|}
\hline $\begin{array}{c}\text { No. } \\
\text { of } \\
\text { wraps }\end{array}$ & $\begin{array}{l}\text { Max. axial } \\
\text { strength, } \\
\text { kN (lbf) }\end{array}$ & $\begin{array}{c}\text { Standard } \\
\text { deviation } \\
\text { (lbf) }\end{array}$ & $\begin{array}{l}\text { Avg. axial } \\
\text { strength, } \\
\text { KN (lbf) }\end{array}$ & $\begin{array}{l}\text { Avg. axial } \\
\text { stress, } \\
\text { MPa (psi) }\end{array}$ & $\begin{array}{l}\text { Avg. \% } \\
\text { increase in } \\
\text { strength }\end{array}$ & $\begin{array}{l}\% \text { strength } \\
\text { increase per } \\
\text { layer }\end{array}$ \\
\hline None & $\begin{array}{l}104.57(23509) \\
112.98(25399) \\
104.57(23509)\end{array}$ & 1091 & $107(24139.5)$ & $13.24(1921)$ & - & - \\
\hline 1 & $\begin{array}{l}167.45(37644) \\
168.38(37853) \\
171.26(38500)\end{array}$ & 446 & 169 (37999.0) & $20.85(3024)$ & 57.4 & 57.4 \\
\hline 2 & $\begin{array}{l}222.49(50017) \\
205.51(46200) \\
214.58(48240)\end{array}$ & 1910 & $214(48152.7)$ & $26.42(3832)$ & 99.5 & 49.8 \\
\hline 3 & $\begin{array}{l}233.70(51538) \\
255.76(57498) \\
242.74(55570)\end{array}$ & 3041 & $244(54869.0)$ & $30.10(4366)$ & 127.3 & 42.4 \\
\hline 5 & $\begin{array}{l}398.08(89493) \\
392.07(88140) \\
361.07(81172)\end{array}$ & 4465 & $384(86268.3)$ & $47.33(6865)$ & 257.4 & 51.5 \\
\hline
\end{tabular}

Note: Overlap length of $50 \mathrm{~mm}-100 \mathrm{~mm}$ (2"-4") was used for 3 and 5 layers of wrapping. It was also observed that the overlap length can influence the amount of strength increase. 
Similarly, compression tests were performed after 7 and 28 days of curing on nonwrapped and wrapped cylinders of similar sizes $100 \mathrm{~mm}$ x $200 \mathrm{~mm}$ (4" x 8") cast with underwater rapid set and high early strength structural mortar (five star structural concrete). Tests were performed after 7 and 28 days of curing and the results are shown in Table 3-5.

Table 3-5: Strength \& \% strength increase of non-wrapped/wrapped cylinders cast with underwater concrete

\begin{tabular}{|c|c|c|c|c|c|c|}
\hline \multirow[b]{2}{*}{$\begin{array}{l}\text { No. } \\
\text { of } \\
\text { wraps }\end{array}$} & \multicolumn{3}{|c|}{ After 7 days of curing } & \multicolumn{3}{|c|}{ After 28 days of curing } \\
\hline & $\begin{array}{l}\text { Max. axial } \\
\text { strength } \\
\text { kN (lbs) }\end{array}$ & $\begin{array}{c}\text { Avg. axial } \\
\text { stress } \\
\text { MPa (psi) }\end{array}$ & $\begin{array}{c}\% \\
\text { increase } \\
\text { in } \\
\text { strength }\end{array}$ & $\begin{array}{l}\text { Max. axial } \\
\text { strength } \\
\text { kN (lbs) }\end{array}$ & $\begin{array}{c}\text { Avg. axial } \\
\text { stress } \\
\text { MPa (psi) }\end{array}$ & $\begin{array}{c}\% \\
\text { increase } \\
\text { in } \\
\text { strength }\end{array}$ \\
\hline 0 & $\begin{array}{l}37.72(8480) \\
34.81(7825) \\
35.19(7910)\end{array}$ & $\begin{array}{l}4.43 \\
(642)\end{array}$ & - & $\begin{array}{l}48.04(10799) \\
50.84(11430) \\
48.75(10960)\end{array}$ & $\begin{array}{l}6.07 \\
(880)\end{array}$ & - \\
\hline 3 & $\begin{array}{l}186.98(42035) \\
205.64(46230) \\
189.34(42565)\end{array}$ & $\begin{array}{l}23.92 \\
(3470)\end{array}$ & 440.3 & $\begin{array}{l}222.59(50040) \\
205.51(46200) \\
198.30(44580)\end{array}$ & $\begin{array}{l}25.75 \\
(3735)\end{array}$ & 324.2 \\
\hline & & $\begin{array}{l}2828 \mathrm{psi} \\
\text { from wrap }\end{array}$ & & & $\begin{array}{l}2855 \mathrm{psi} \\
\text { from wrap }\end{array}$ & \\
\hline
\end{tabular}

\subsubsection{Concrete Compressive Strength}

Plain Concrete cylinders wrapped underwater with single layer of Aquawrap showed an average strength increase of 57.4\% (Table 3-4). Similarly, 2, 3 and 5 layers of wrapping resulted in average percent increase in strength per layer by $49.8 \%, 42.4 \%$ and $51.5 \%$, respectively. Strength increase per layer ranged from $42.4 \%$ to $57.4 \%$ and average strength increase per layer was noted to be $\sim 50 \%$. The average percentage axial compressive strength increase for $1,2,3$, and 5 layers of wrapping is shown in Figure 3.14. 
Similarly, average percentage increase in axial compressive strength for cylinders cast with underwater rapid set, high early strength concrete (five star structural concrete) after 28 days of curing was obtained to be $324 \%$ for 3 layers of wrapping, which comes to around $108 \%$ increase for single layer of wrapping. This huge increment is for the fact that, the unconfined concrete strength itself is too low (880 psi). The extra axial compressive strength obtained on confined concrete cylinders wrapped with certain number of layers of wrap comes to be same $(\sim 12,000 \mathrm{lbs})$. For lower unconfined strength concrete, $\%$ increase in axial compressive strength is seen more due to the denominator being smaller. Increase in axial strength due to wrapping is higher with initial number of layers but with increasing number of layers of wrapping, \% increase in axial strength will not be significant compared to the initial layers.

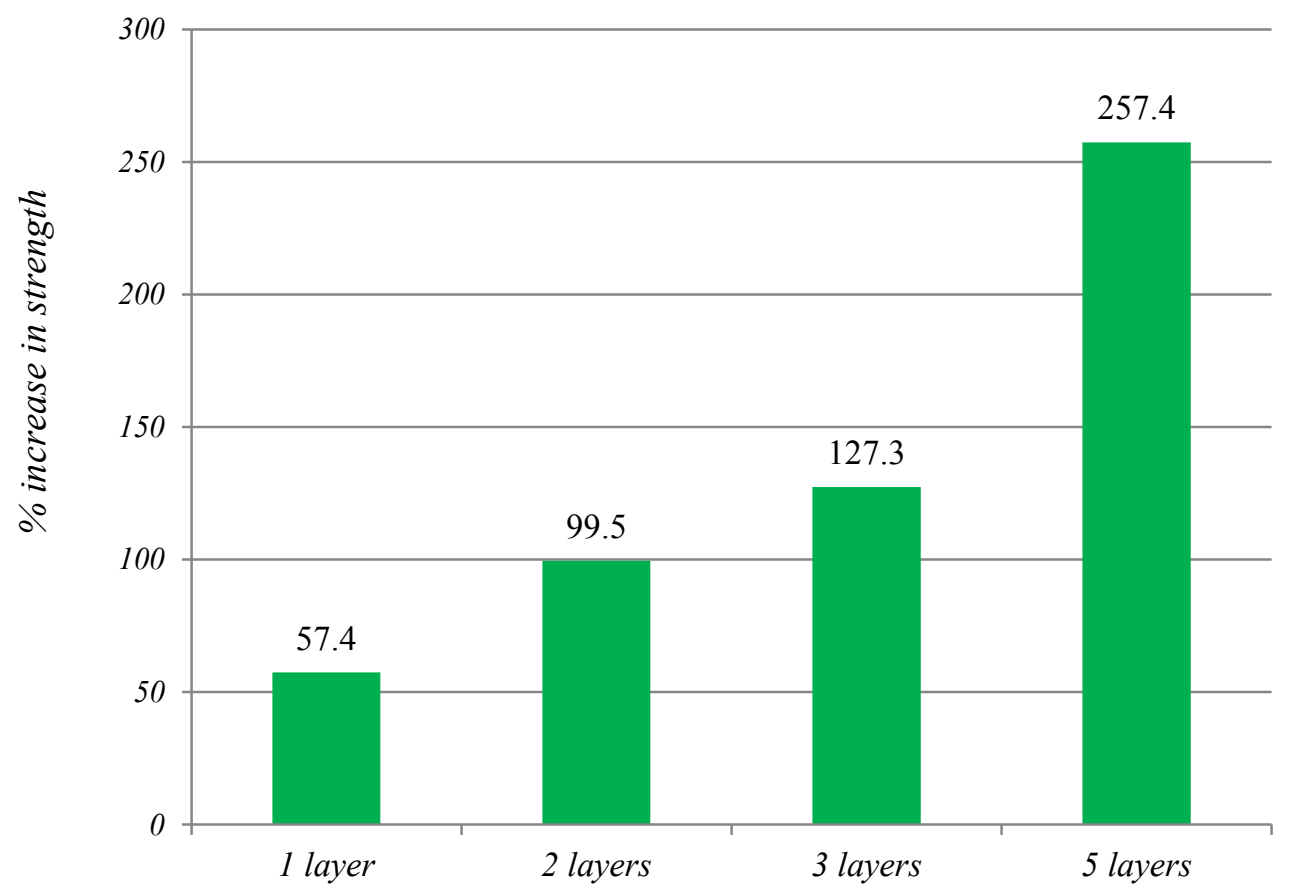

Figure 3.14: Average axial strength increase for 1, 2, 3, and 5 layers of wrapping 


\subsubsection{Stiffness}

The stiffness of non-wrapped and wrapped specimens were calculated from their loaddeflection $(\mathrm{p}-\delta)$ graph respectively. As we know, stiffness is a property of a structure and for an element in tension or compression and is computed by the equation:

$$
k=\frac{A E}{L}=\frac{P}{\delta}
$$

From $\mathrm{p}-\delta$ graph (Figure 3.15), maximum stiffness of the unconfined concrete cylinder comes to be $14098 \mathrm{~N} / \mathrm{mm}$. Similarly, maximum stiffness of the confined concrete cylinders with 1, 2, 3, and 5 layers of wrapping come to be $21321 \mathrm{~N} / \mathrm{mm}, 27282 \mathrm{~N} / \mathrm{mm}, 30317 \mathrm{~N} / \mathrm{mm}$, and $37714 \mathrm{~N} / \mathrm{mm}$ respectively. This shows an increase in stiffness of wrapped cylinders with 5 layers of wrapping by 2.67 times compared to the non-wrapped ones. The stiffness increase by $51 \%$ is seen after the first layer of wrapping while the stiffness increase by $28 \%$ is seen after the second layer of wrapping. After the third layer of wrapping and so on, $\%$ increase in stiffness is seen to be decreasing as compared to the $\%$ increase in stiffness achieved in initial layers of wrapping.

\subsubsection{Energy Absorbed}

Further, increasing area under load vs. deflection curve (Figure 3.15) shows an increasing amount of ductility and energy absorption for concrete cylinders wrapped with 1, 2, 3 and 5 layers of GFRP Aquawrap. The energy absorbed until the failure of the concrete cylinder specimens computed from the area under the load-deflection curve (Figure 3.15) is given in Table 3-6. It can be observed that the energy absorbed after the first layer of wrapping was $63 \%$ more than the energy absorbed on unwrapped concrete cylinders. Similarly, energy absorbed after 2, 3, and 5 layers of wrapping is $105 \%, 192 \%$, and $322 \%$ respectively, more than the energy 
absorbed on the non-wrapped ones. This concludes the increase in ductility and energy absorption of the concrete cylinders due to wrapping.

Table 3-6: Energy absorbed by concrete cylinders until failure

\begin{tabular}{|c|c|}
\hline No. of wrap layers on specimens & Energy absorbed (Joules) \\
\hline 0 wrap & 1240.17 \\
\hline 1 wrap & 2029.93 \\
\hline 2 wraps & 2552.94 \\
\hline 3 wraps & 3627.05 \\
\hline 5 wraps & 5234.25 \\
\hline
\end{tabular}

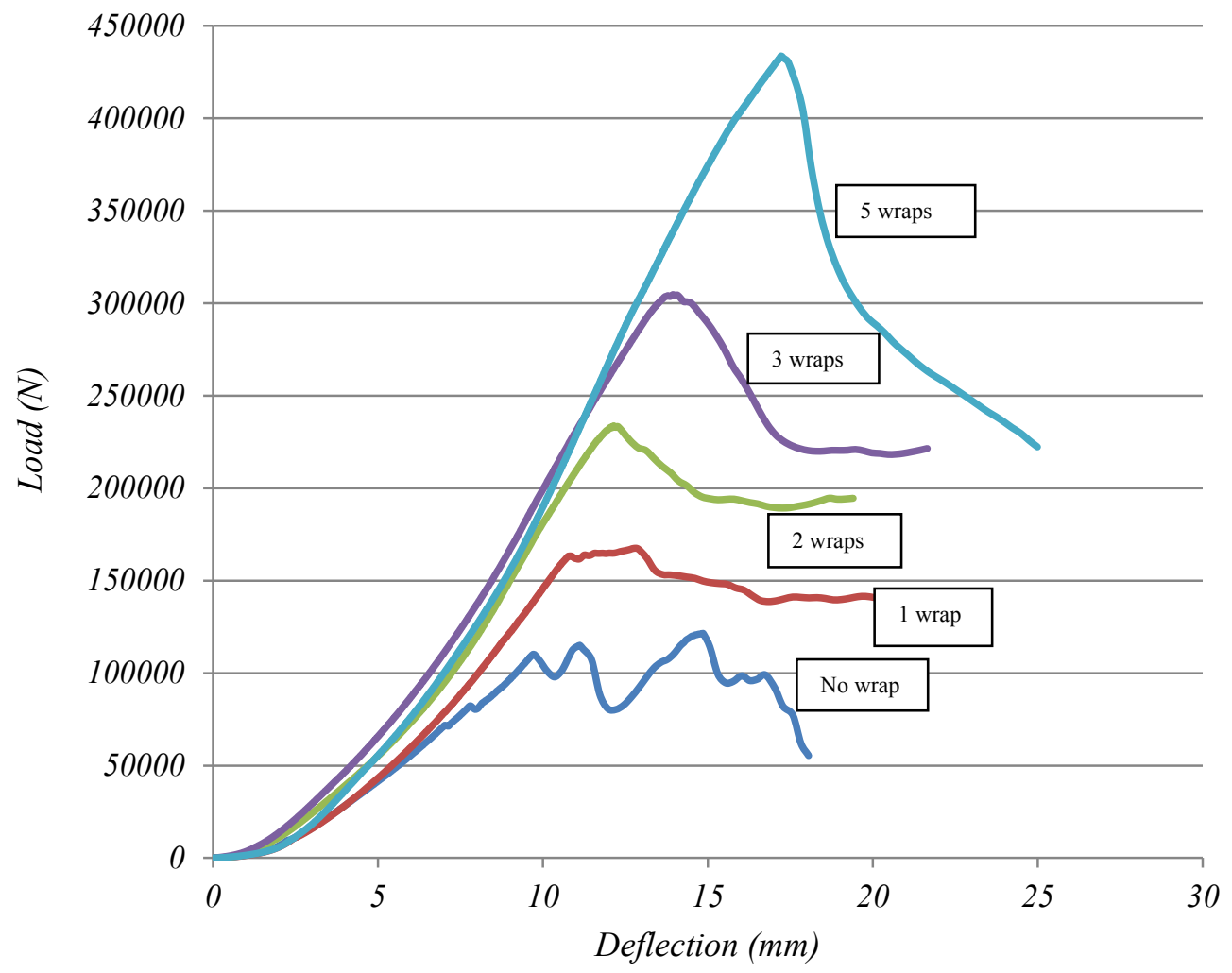

Figure 3.15: Load vs. axial deformation graphs for cylinders with 1, 2, 3 and 5 wraps 


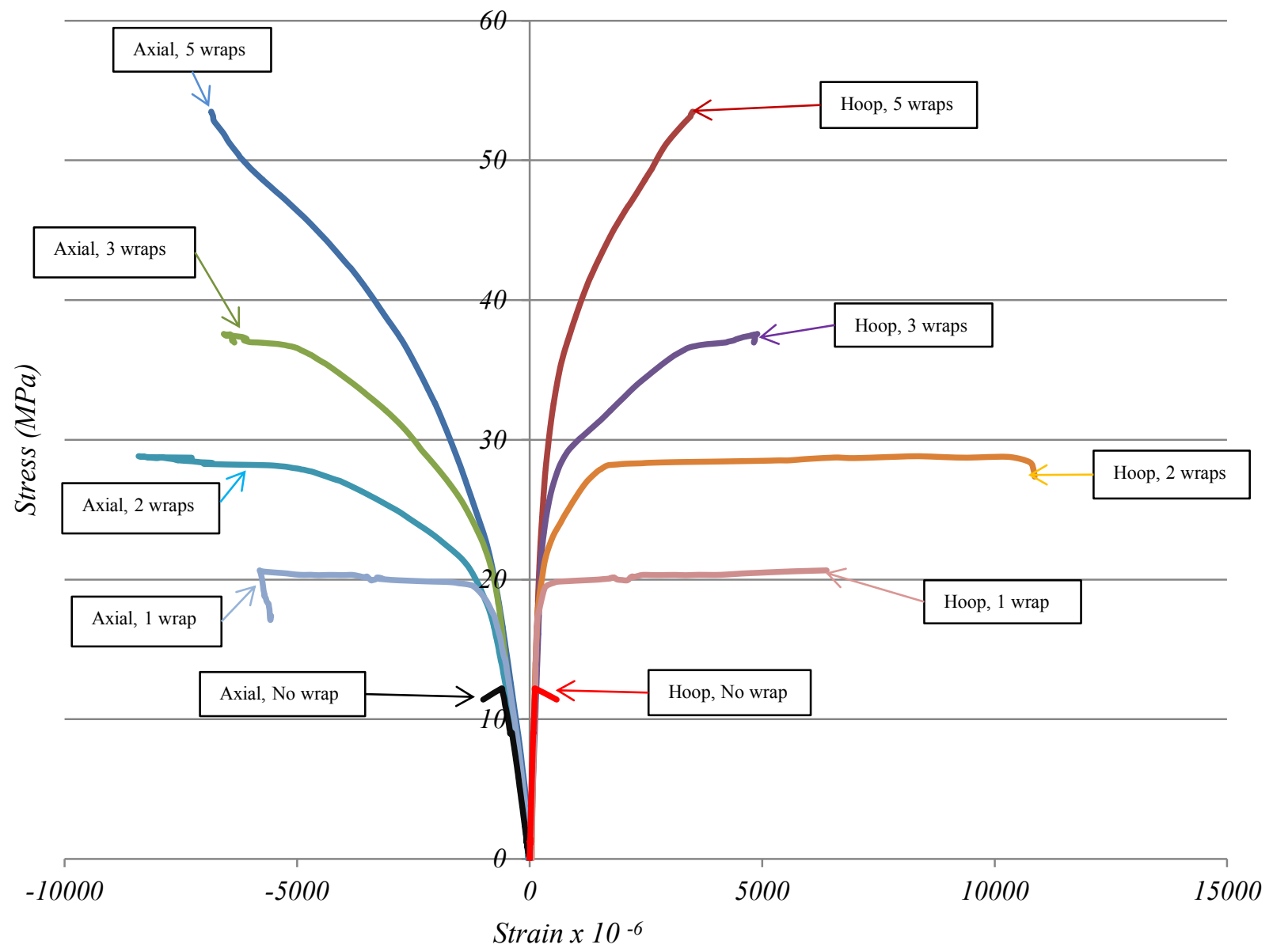

Figure 3.16: Stress vs. axial, hoop strains for 1, 2, 3, and 5 wraps.

(Note: readings may have been discontinued due to strain gage failure before cylinder rupture).

\subsection{Theoretical Analysis}

The confined strengths of cylindrical concrete with FRP wrapping obtained through experiments have been correlated with different analytical models given by different researchers (Table 3-7). Different confined strength values of concrete cylinders wrapped with 1,2, 3, and 5 layers of fabrics are computed using five different models shown in Table 3-7. As per the computations, theoretical confined strengths agreed well with the experimental values. 
Table 3-7: Analytical models used for correlation

\begin{tabular}{|c|c|c|}
\hline Model no. & \multicolumn{1}{|c|}{ Authors } & Modeling Equations \\
\hline M1. & $\begin{array}{l}\text { Lam and Teng, 2003 [6] } \\
\text { (ACI 440.2R-08) }\end{array}$ & $\frac{f_{c c}^{\prime}}{f_{c o}^{\prime}}=1+3.3 \frac{f_{l}}{f_{c o}^{\prime}}$ \\
\hline M2. & Matthys et al. 2006 [27] & $\frac{f_{c c}^{\prime}}{f_{c o}^{\prime}}=1+2.3 \frac{f_{l}}{f_{c o}^{\prime}}$ \\
\hline M3. & Mander et al. 1988 [28] & $\frac{f_{c c}^{\prime}}{f_{c o}^{\prime}}=-1.254+2.254$ \\
\hline M4. & Toutanji, 1999 [29] & $\frac{7.94 f_{l}}{f_{c o}^{\prime}}-2{\frac{f_{l}}{f_{c o}^{\prime}}}^{0.85}$ \\
\hline
\end{tabular}

Where,

$f_{c c}^{\prime}=$ compressive strength of confined concrete

$f_{c o}^{\prime}=$ compressive strength of unconfined concrete $=13.2 \mathrm{MPa}(1921 \mathrm{psi})$

$f_{l}=$ lateral confined pressure by GFRP wraps $=\frac{2 f_{f r p} t_{f}}{D}$

Or, $f_{f r p}=E_{f} n \epsilon_{f u}$, Where,

$E_{f}=$ modulus of elasticity of GFRP Aquawrap $=15.7 \mathrm{GPa}$ (2.28 msi)

$n=$ number of layers $=1,2,3$, and 5

$t_{f}=$ thickness of fabric $=1 \mathrm{~mm}\left(0.0285^{\prime \prime}\right)$

$\epsilon_{f u}=$ ultimate failure strain of fabric $=15,000 \times 10^{-6}$ (from test results) 
In ACI 440.2R-08, $\epsilon_{f e}=$ effective failure strain of fabric $=k_{\epsilon} \epsilon_{f u}$

Where, $k_{\epsilon}=$ strain efficiency factor $=0.55$

Table 3-8: Comparisons of experimental \& theoretical confined strength of 4" diameter concrete cylinders

\begin{tabular}{|c|c|c|c|c|c|c|c|c|c|c|c|c|c|}
\hline \multirow{2}{*}{$\begin{array}{c}\text { No. } \\
\text { of } \\
\text { wraps }\end{array}$} & \multicolumn{4}{|c|}{$\begin{array}{c}\left(f_{l}\right) \\
\operatorname{MPa}(\mathrm{psi})\end{array}$} & \multirow{2}{*}{$\begin{array}{c}f_{c c \text { expt }}^{\prime} \\
\mathrm{MPa} \\
(\mathrm{psi})\end{array}$} & \multicolumn{4}{|c|}{$\begin{array}{l}f_{c c}^{\prime} \text { theory } \\
\mathrm{MPa}(\mathrm{psi})\end{array}$} & \multicolumn{4}{|c|}{$\frac{f_{c c \text { expt }}^{\prime}}{f_{c c}^{\prime} \text { theory }}$} \\
\hline & M1 & M2 & M3 & M4 & & M1 & M2 & M3 & M4 & M1 & M2 & M3 & M4 \\
\hline 1 & $\begin{array}{l}1.85 \\
(268)\end{array}$ & $\begin{array}{l}2.01 \\
(292)\end{array}$ & $\begin{array}{c}2.52 \\
(366)\end{array}$ & $\begin{array}{l}2.01 \\
(292)\end{array}$ & $\begin{array}{l}20.85 \\
(3024)\end{array}$ & $\begin{array}{l}19.35 \\
(2806)\end{array}$ & $\begin{array}{l}19.39 \\
(2813)\end{array}$ & $\begin{array}{l}25.66 \\
(3721)\end{array}$ & $\begin{array}{l}22.60 \\
(3278)\end{array}$ & 1.08 & 1.07 & 0.81 & 0.92 \\
\hline 2 & $\begin{array}{l}3.70 \\
(536)\end{array}$ & $\begin{array}{l}4.03 \\
(585)\end{array}$ & $\begin{array}{l}5.04 \\
(731)\end{array}$ & $\begin{array}{l}4.03 \\
(585)\end{array}$ & $\begin{array}{l}26.42 \\
(3832)\end{array}$ & $\begin{array}{l}25.44 \\
(3690)\end{array}$ & $\begin{array}{l}24.33 \\
(3529)\end{array}$ & $\begin{array}{l}33.18 \\
(4812)\end{array}$ & $\begin{array}{l}30.12 \\
(4368)\end{array}$ & 1.04 & 1.08 & 0.80 & 0.88 \\
\hline 3 & $\begin{array}{l}5.54 \\
(804)\end{array}$ & $\begin{array}{l}6.05 \\
(877)\end{array}$ & $\begin{array}{c}7.56 \\
(1097)\end{array}$ & $\begin{array}{l}6.05 \\
(877)\end{array}$ & $\begin{array}{l}30.10 \\
(4366)\end{array}$ & $\begin{array}{l}31.54 \\
(4575)\end{array}$ & $\begin{array}{l}28.89 \\
(4190)\end{array}$ & $\begin{array}{l}38.49 \\
(5582)\end{array}$ & $\begin{array}{l}37.05 \\
(5374)\end{array}$ & 0.95 & 1.04 & 0.78 & 0.81 \\
\hline 5 & $\begin{array}{c}9.24 \\
(1340)\end{array}$ & $\begin{array}{l}10.08 \\
(1462)\end{array}$ & $\begin{array}{l}12.60 \\
(1828)\end{array}$ & $\begin{array}{l}10.08 \\
(1462)\end{array}$ & $\begin{array}{l}47.33 \\
(6865)\end{array}$ & $\begin{array}{l}43.74 \\
(6344)\end{array}$ & $\begin{array}{l}37.40 \\
(5424)\end{array}$ & $\begin{array}{l}45.51 \\
(6600)\end{array}$ & $\begin{array}{l}50.00 \\
(7252)\end{array}$ & 1.08 & 1.26 & 1.04 & 0.95 \\
\hline
\end{tabular}

The experimental results obtained from the testing of water-curable FRP confined concrete cylinders correlated well or slightly higher with results obtained from the theoretical analytical models as shown in Table 3-8. The experimental results were best matched with the model used by ACI 440.2R-08, as it uses strain efficiency factor of 0.55 , which accounts for premature failure of FRP under multi-axial state of stress [30]. Also, the model accounts for the hoop rupture strain of FRP rather than the ultimate material tensile strain where, hoop strain is always lower than the material ultimate tensile strength.

\subsection{Failure Mode}

Non-wrapped concrete cylinders, i.e. plain concrete cylinders tested under compression failed in a brittle manner. Small chunks of concrete were broken at the bottom and top of the cylinders at approximately $45^{0}$ indicating compression-shear failure of the specimens. However, in non-wrapped cylinders cast with underwater rapid set, early strength structural concrete failed 
in longitudinal direction with cracks propagating from one end to the other. This might be due to the absence of coarse aggregates in the concrete in order to arrest the propagation of the cracks. However, this failure mode is of no significance as it is not a true concrete cylinder and for the fact that specimens were prepared to see the confinement effects of the wrap.

Wrapped concrete cylinders failed near the top and bottom under axial compression in a ductile manner. Most concrete cylinders with wrap under compressive loads failed by tensile rupture of the wrap after the concrete had been disintegrated. Just prior to failure, specimens exhibited significant lateral dilatation mostly at the top and bottom region. This circumferential (lateral) dilatation was produced due to the confinement effect of surrounding FRP layers. With increasing circumferential stress and deformations, the wraps finally ruptured at the region of dilatation i.e. at the top and bottom, leading to the ultimate failure of specimens (Figure 3.17). However, in some of the confined specimens, ultimate failure was noticed due to the de-bonding of the wrap at the region of overlap i.e. due to the separation of the overlapped joint.

Similarly, with the specimens prepared from underwater rapid set-high early strength concrete (five star structural concrete), failure modes of the confined cylinder were similar. The lateral dilatation was seen at the top and bottom region after the deterioration of concrete and finally, the specimen was failed due to the tensile rupture of the wrap at the dilated region (Figure 3.18). 

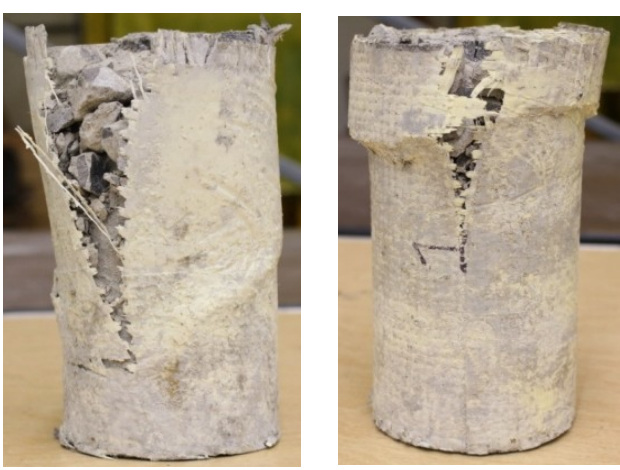

Single layer
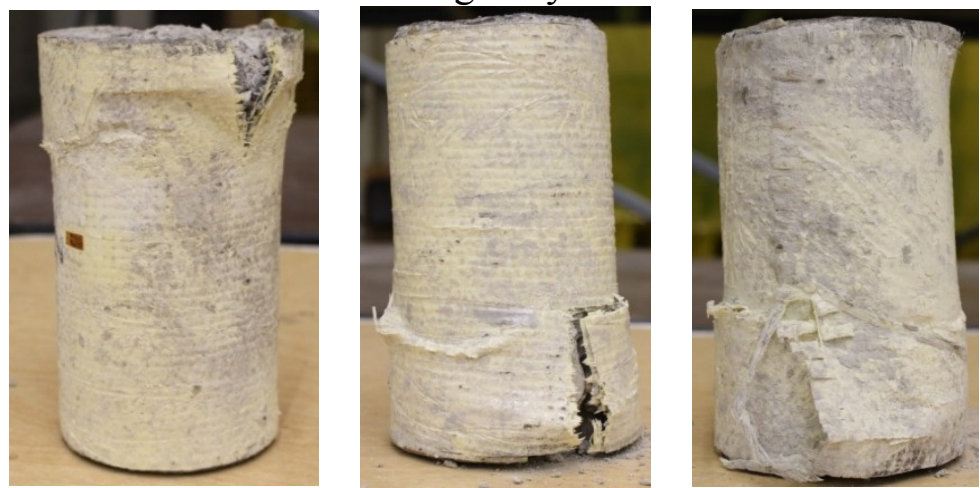

2 layers
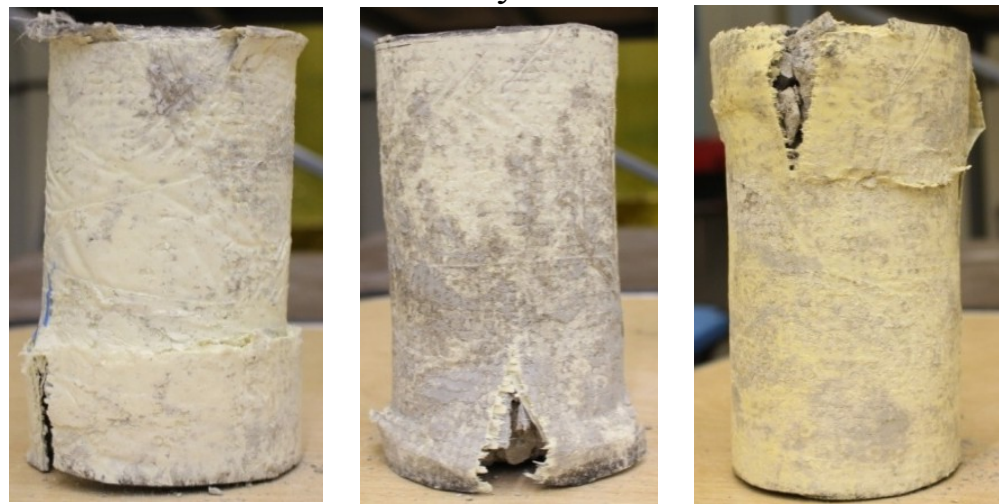

3 layers
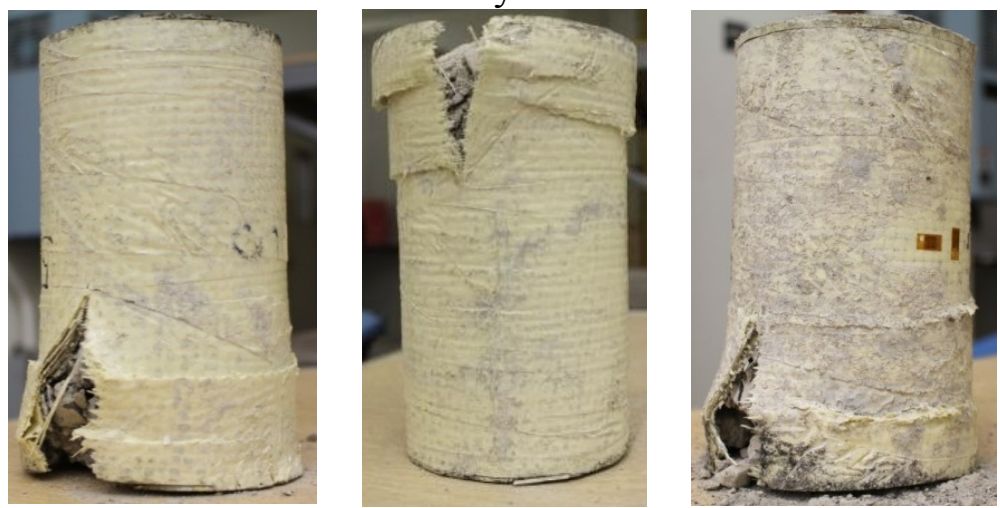

5 layers

Figure 3.17: Failure mode of wrapped concrete cylinders with 1, 2, 3 and 5 layers of wrapping 

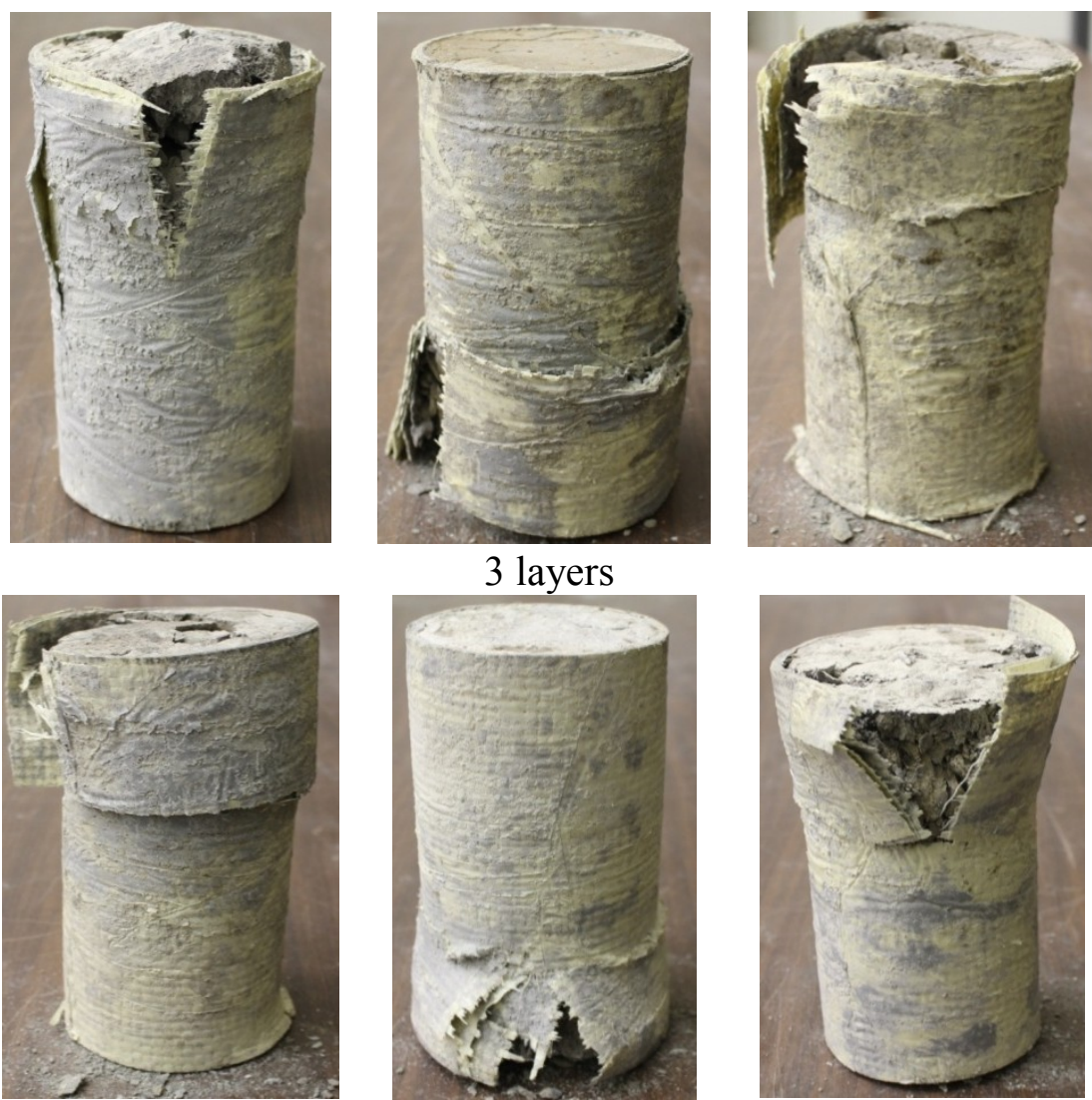

5 layers

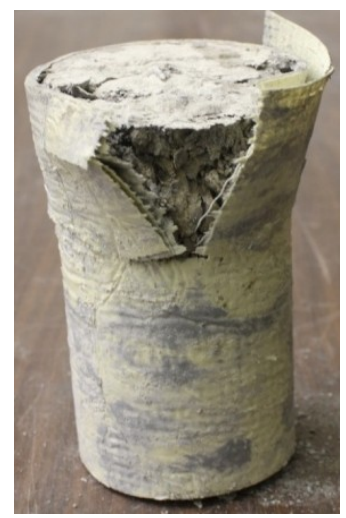

Figure 3.18: Failure mode of wrapped cylinders (five star structural concrete) with 3 and 5 layers of wrapping

\subsection{Conclusions}

This study focused on the structural performance of concrete cylinders confined with water curable Aquawrap repair system. Based on the study, following conclusions can be stated.

1. The underwater FRP repair system provided good confinement for concrete cylinders which was evidenced as the cylinders with 1, 2, 3, and 5 layers of GFRP Aquawrap showed per layer strength increase of $57.4 \%, 49.8 \%, 42.4 \%$, and $51.5 \%$, respectively, giving an average strength increase of $\sim 50 \%$ per layer of wrapping. 
2. The maximum stiffness increase after the first layer of wrapping was $51 \%$ and the maximum stiffness further increased by $28 \%$ after the second layer of wrapping. After the third layer of wrapping and so on, percentage increase in stiffness was seen less compared to the $\%$ increase in stiffness achieved in the initial layers of wrapping. This showed an increase in maximum stiffness of wrapped cylinders with 5 layers of wrapping by 2.67 times compared to the non-wrapped ones.

3. Increasing area under load vs. axial deformation as well as from stress vs. strain curve for varying number of GFRP wrap layers (1 to 5) showed an increase in ductility and energy absorption. It was observed that the energy absorbed after the first layer of wrapping was $63 \%$ more than the energy absorbed on unwrapped concrete cylinders. Similarly, energy absorbed after 2, 3, and 5 layers of wrapping was $105 \%, 192 \%$, and $322 \%$ respectively, more than the energy absorbed on the non-wrapped ones.

4. The experimental confined strength values correlated well within $8 \%$ as per ACI $440.2 \mathrm{R}-$ 08 FRP confinement equation. Further, the predicted confined strength values from different confinement models proposed by different authors resulted similar to the experimental results. 


\section{CHAPTER 4 REHAB OF CONCRETE DISCHARGE PORTS IN}

\section{CHICKAMAUGA DAM WITH FRP}

\subsection{Introduction}

Chickamauga Lock is seven miles northeast of Chattanooga at Tennessee River Mile 471. The 60 feet $\times 360$ feet lock has been in operation since 1940. Figure 4.1 shows the view of the dam and lock in Chickamauga. The lock suffered from the Alkali Aggregate Reaction (AAR) from its early stage. AAR is a chemical reaction that causes concrete to absorb water and grow at an uncontrolled rate. The growth results in structural stresses and equipment breakdowns which might threaten the continued structural stability and operation of the navigation lock. If the problem is not solved, the existing lock will eventually be closed causing the navigable upstream of Chickamauga lock isolated from the inland waterways system. This would present difficulties in transporting materials to upstream industries (TVA nuclear power plants, US department of energy facilities etc.) [31].

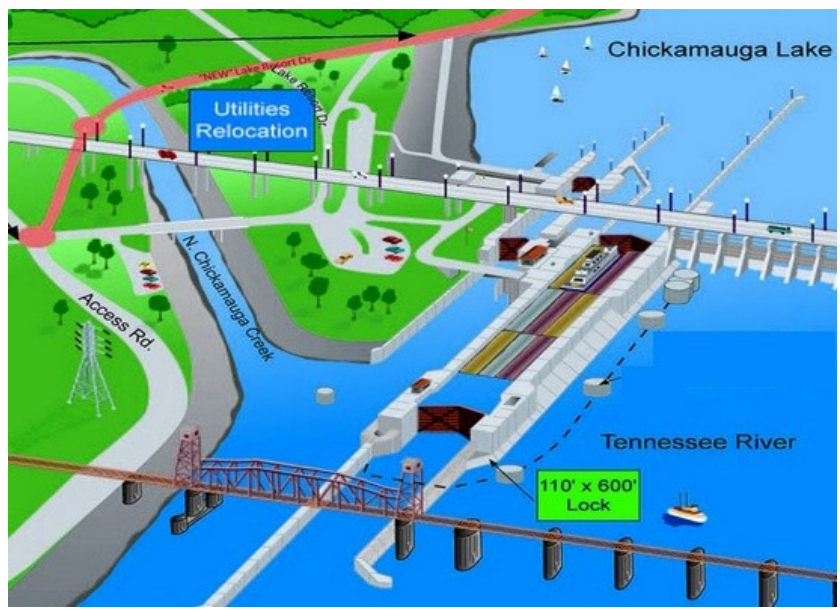

Figure 4.1: Old lock (60 ft. x $360 \mathrm{ft}$.$) and new lock (110 ft. x 660 \mathrm{ft}$.$) in Chickamauga dam, TN$ 
After being opened to navigation, the main section of Chickamauga lock has grown 12 inches in length on the riverside and more than seven inches on the landside [32]. This expansion of concrete, occurred due to a chemical reaction between the alkali in the cement and the minerals in the stone, has caused deterioration of concrete and further the erosive effects from hydraulic forces have caused large chunks of concrete to break into loose pieces. The growing concrete at the lock has created many problems. In some places large chunks of concrete have broken loose from the lock walls. And as the massive blocks that make up the lock have expanded at different rates, the top of the structure is uneven. The lock has actually grown five inches inside the lock chamber lengthwise.

In July, 2012, 15 columns dividing the discharge ports were repaired with conventional methods using steel wrap with grout filler which did not work to full satisfaction, therefore in order to achieve better performance, save installation time and material costs, the use of FRP wraps has been tried out in order to prevent tension failures as well as loss and damage of concrete in discharge ports in Chickamauga dam, Tennessee (Figs. 1, $2 \&$ 3). Further, as the lock couldn't be dewatered, the discharge ports were completely submerged and the FRP repair work was to be done completely underwater. Therefore, underwater curable Aquawrap repair system was used for the rehabilitation of the discharge ports after the preliminary testing in the lab here at CFC-WVU. 


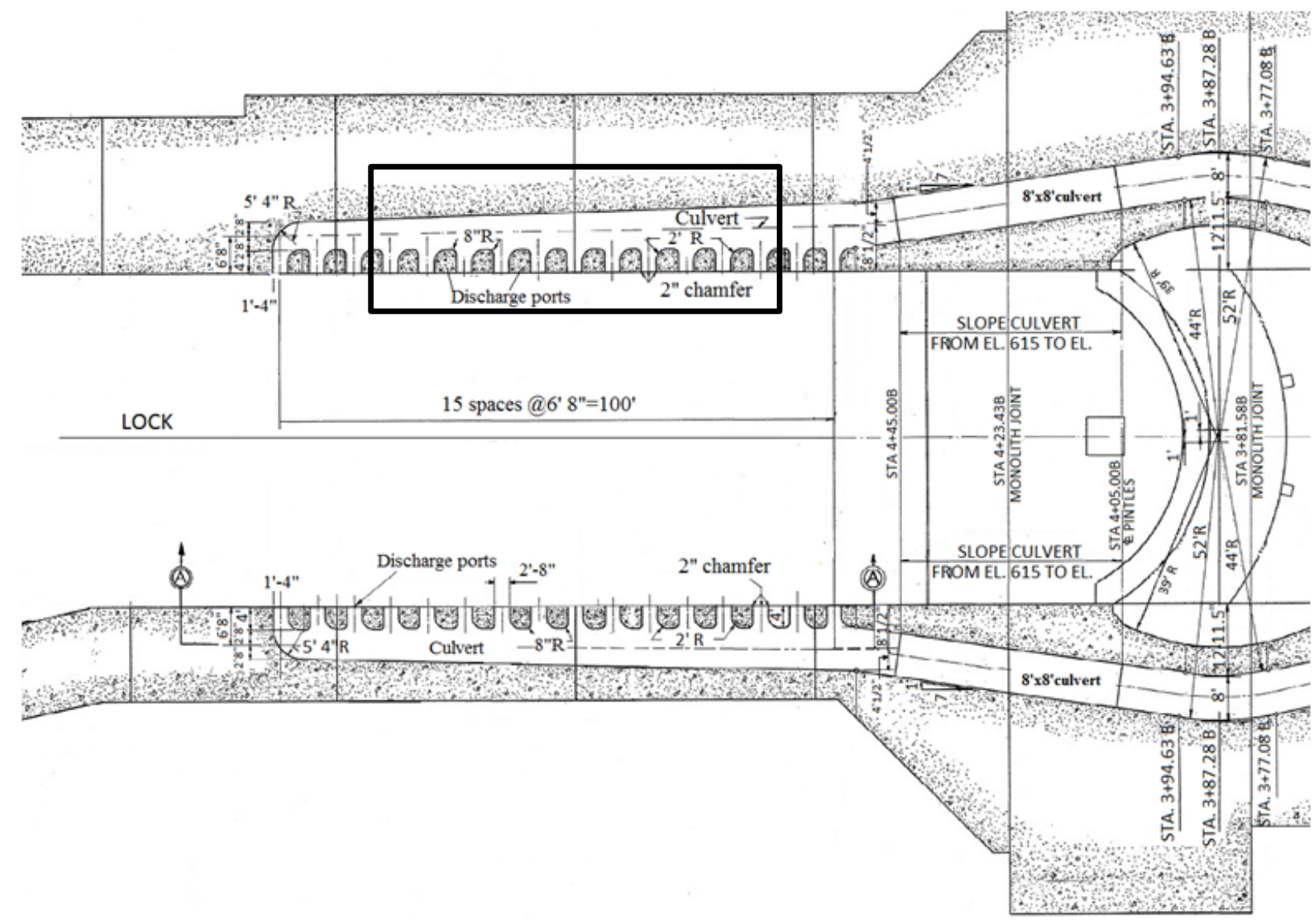

Figure 4.2: Plan view of discharge ports at Chickamauga lock and dam, TN

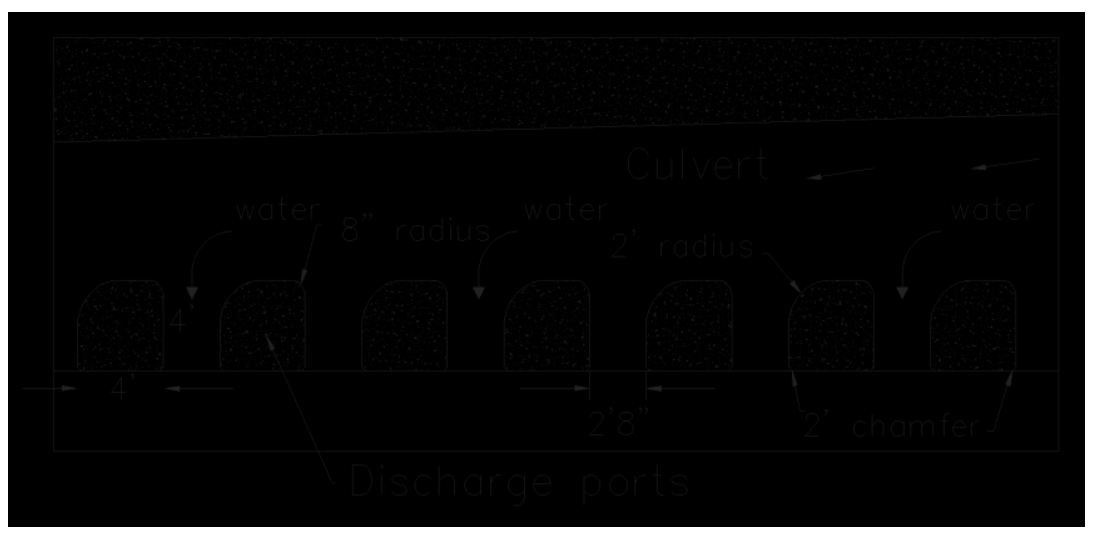

Figure 4.3: Selected view of discharge ports 


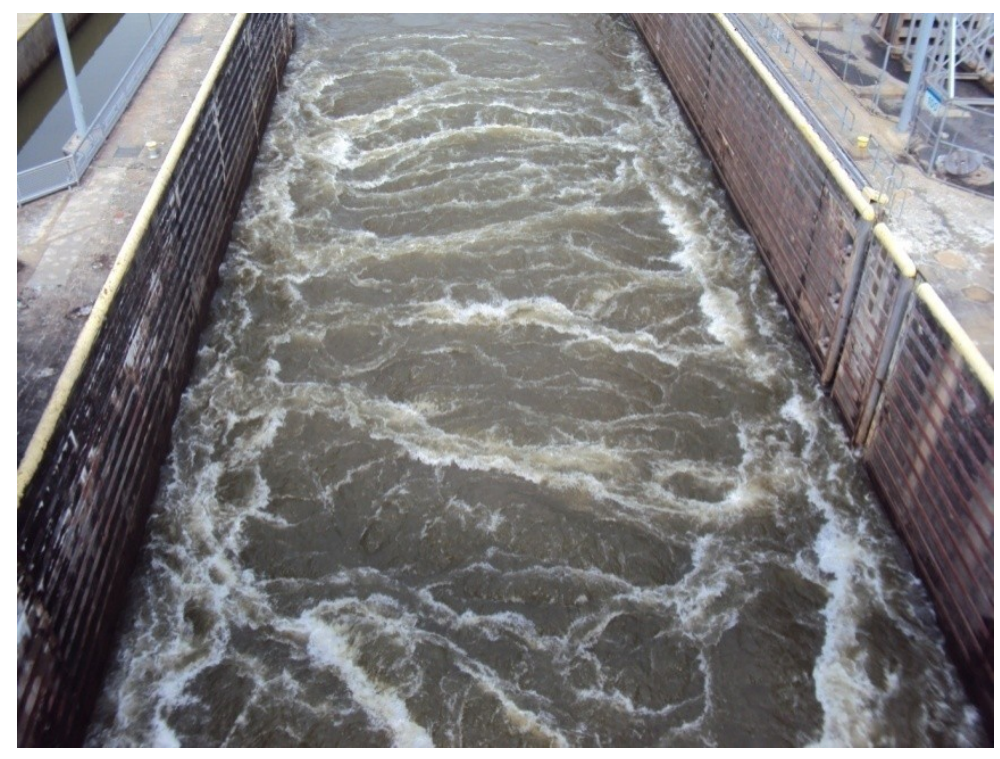

Figure 4.4: View of water released through discharge ports at the Chickamauga lock and dam

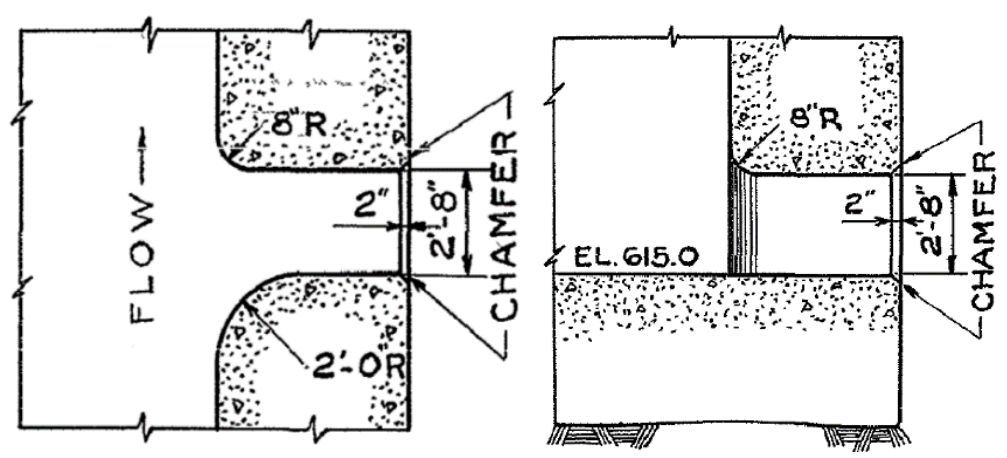

Figure 4.5: Horizontal and vertical sections of discharge ports at Chickamauga lock and dam, TN

\subsection{Field Installation}

\subsubsection{Surface Preparation}

The preparation of the surface of the substrate prior to wrapping is the most crucial step. As discharge ports were submerged and due to the impossibility of dewatering, there were challenges for the repair team to prepare the concrete surface underwater. Entire surface has to be smooth with no cavities and protrusions, with no loose scales due to which stress concentration can be developed \& bonding may be compromised. All the biological growth like 
freshwater algae, fungi were scraped and the entire surface was power washed with the water jet cleaning methods or powered hand tools with rotary cleaning attachments (steel brushes/abrasive discs). The surface was made free from oil/grease so that primer could stick onto the surface of the concrete. The cavities or cracks or any surface depressions or discontinuities were filled with underwater curable grout/concrete and finally made smooth with even surface texture to provide good adhesion to the subsequent layer of wrap.

\subsubsection{Wrapping of the Discharge Ports}

After the surface preparation, a layer of polyurethane primer/adhesive system (BP-4) was mixed and then applied over the entire surface of the pier-like concrete discharge ports in order to provide good bonding of the concrete surface and the Aquawrap layer [32]. The deteriorated discharge ports in Chickamauga lock \& dam were wrapped with Aquawrap repair system underwater during July 15 to 19,2013 . The complete repair, that included preparation of concrete surface, application of primer/adhesive and finally wrapping the structure all around, had to be performed completely underwater by the team of divers. Finally, the pier-like concrete structures were wrapped with two layers of Aquawrap G-05 fabric. After the wrapping was done, epoxy based primer/adhesive was used to coat over the wrap to seal the system after the hardening of Aquawrap. The field wrapping of the discharge ports are shown in Figure 4.6, 4.7 and 4.8 in a sequence.

After three months of installation, the inspection team of divers monitored and examined the wrapped discharge ports. The materials and installation condition looked intact, the same as the day it was put in, as reported by the inspection team [32]. 

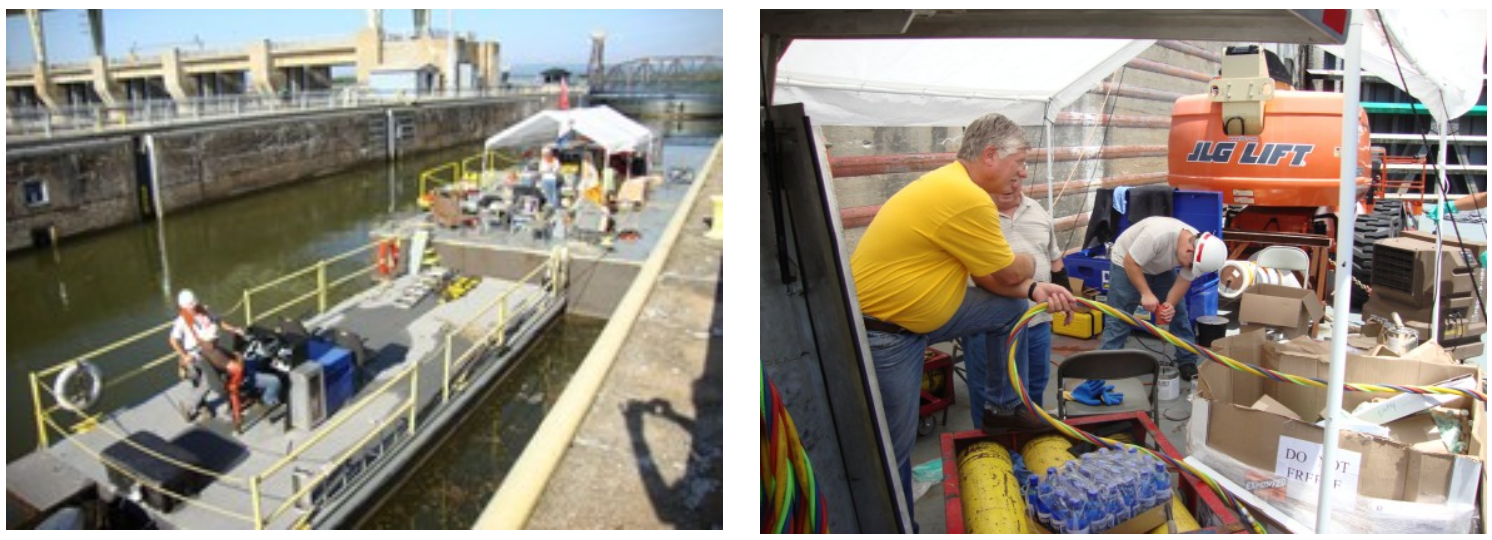

Figure 4.6: Boats with rehab materials, crew, diving support and monitoring system; Resins being mixed on boat;
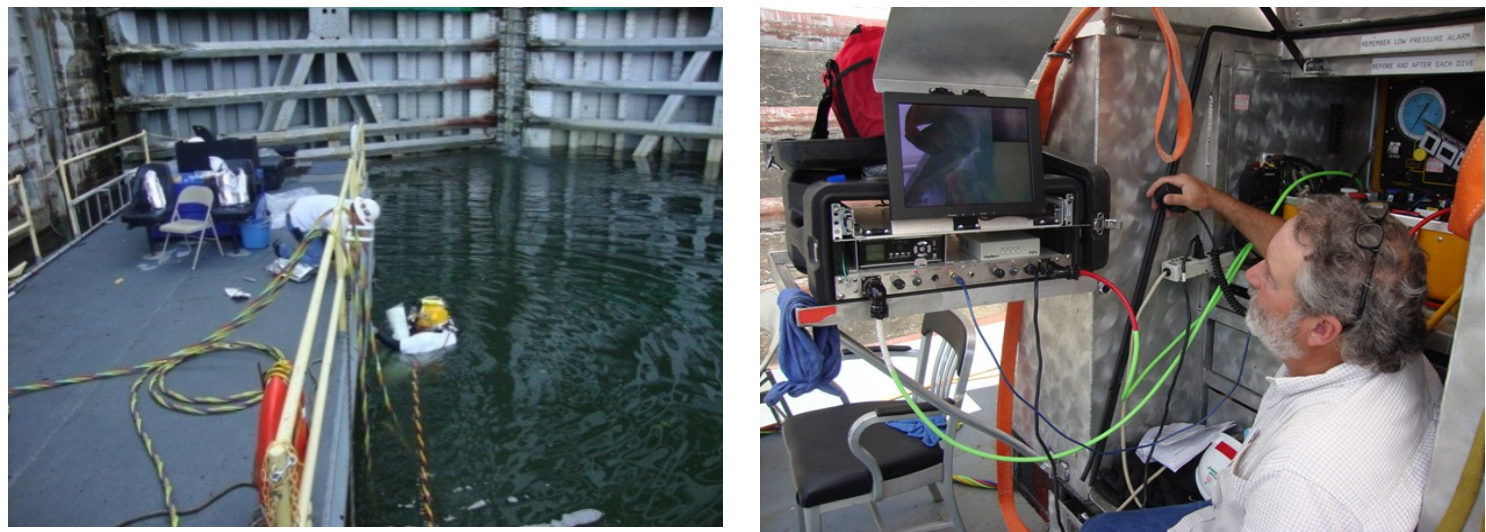

Figure 4.7: Resin saturated FRP fabric roll being passed onto diving team member, Onboard audio-video and depth monitoring
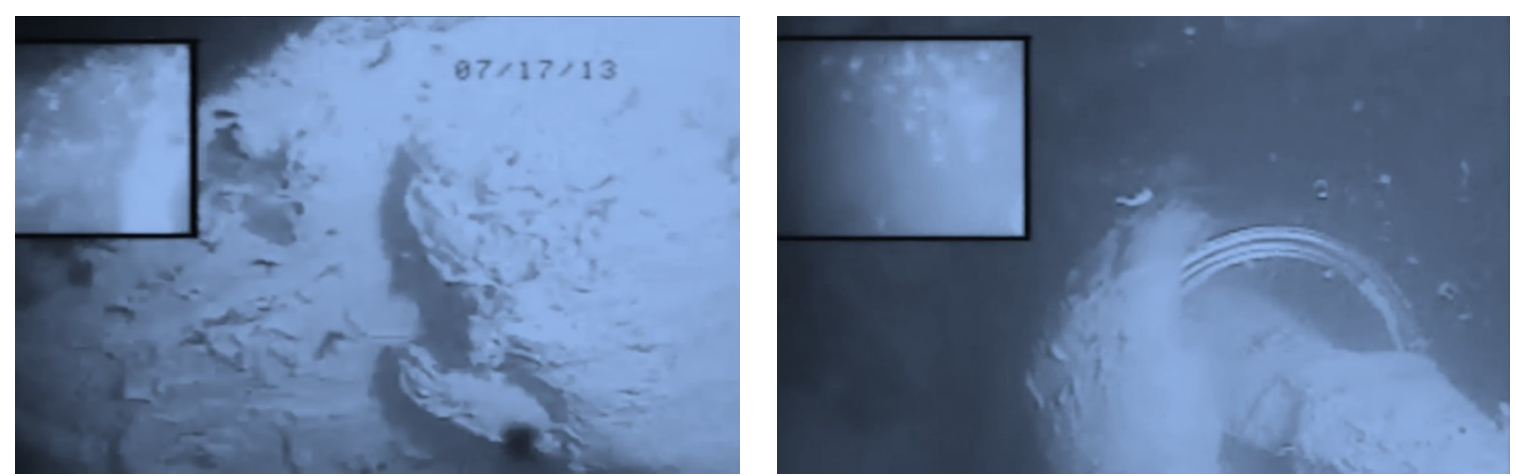

Figure 4.8: Hand application of primer on discharge ports 


\subsection{Conclusions}

Based on the rehabilitation work conducted in discharge ports at Chickamauga dam, following conclusions are drawn:

1. Aquawrap repair system was field implemented successfully with the help of a team of divers at a depth of $\sim 20 \mathrm{ft}$. in discharge ports of Chickamauga dam, TN. As reported by the diving team, the materials and the bond integrity were visually inspected underwater and found to be excellent, three months after the installation.

2. Water curable FRP repair system (Aquawrap) provided an economical solution for underwater repair of concrete structures. It is noted that the surface preparation of the submerged concrete structures is important to get better bonding between FRP system and the substrate. 


\section{CHAPTER 5 REHAB OF STEEL PILES IN EAST LYNN LAKE BRIDGE}

\subsection{Introduction}

East Lynn Lake Bridge is located at Cove Creek, which is a tributary of the East Fork of Twelve pole Creek, West Virginia. It was designed in 1969 and constructed in early 1970s. It consists of 5 spans, and is a two lane, continuous reinforced concrete slab structure designed for H-15-44 loading. Each of the two end spans are 20'-3" in length and the 3 intermediate spans are 27'-6" each. The bridge is supported by four piers and two abutments. The piers and abutments consist of a five pile bent with a reinforced concrete cap with all of the piles founded on bed rock. The existing steel piles are exposed to year round water stream level fluctuations and are susceptible to continued oxidation, rusting, and scouring (Figure 5.1). Under this work, steel Hpiles with advanced deterioration and section loss verified to be up to $60 \%$, will be rehabilitated with FRP jackets and self-consolidating concrete (SCC).

The 40-year-old bridge spanning the East Fork arm of East Lynn Lake had been reduced to one traffic lane, a six-ton weight limit and a 10-mph speed limit because of severely corroded steel support pilings. Officials with the U.S. Army Corps of Engineers' Huntington District, which manages East Lynn Lake, were considering closing the span after structural engineers found that up to 60 percent of some of the steel pilings supporting it had corroded away [33]. 


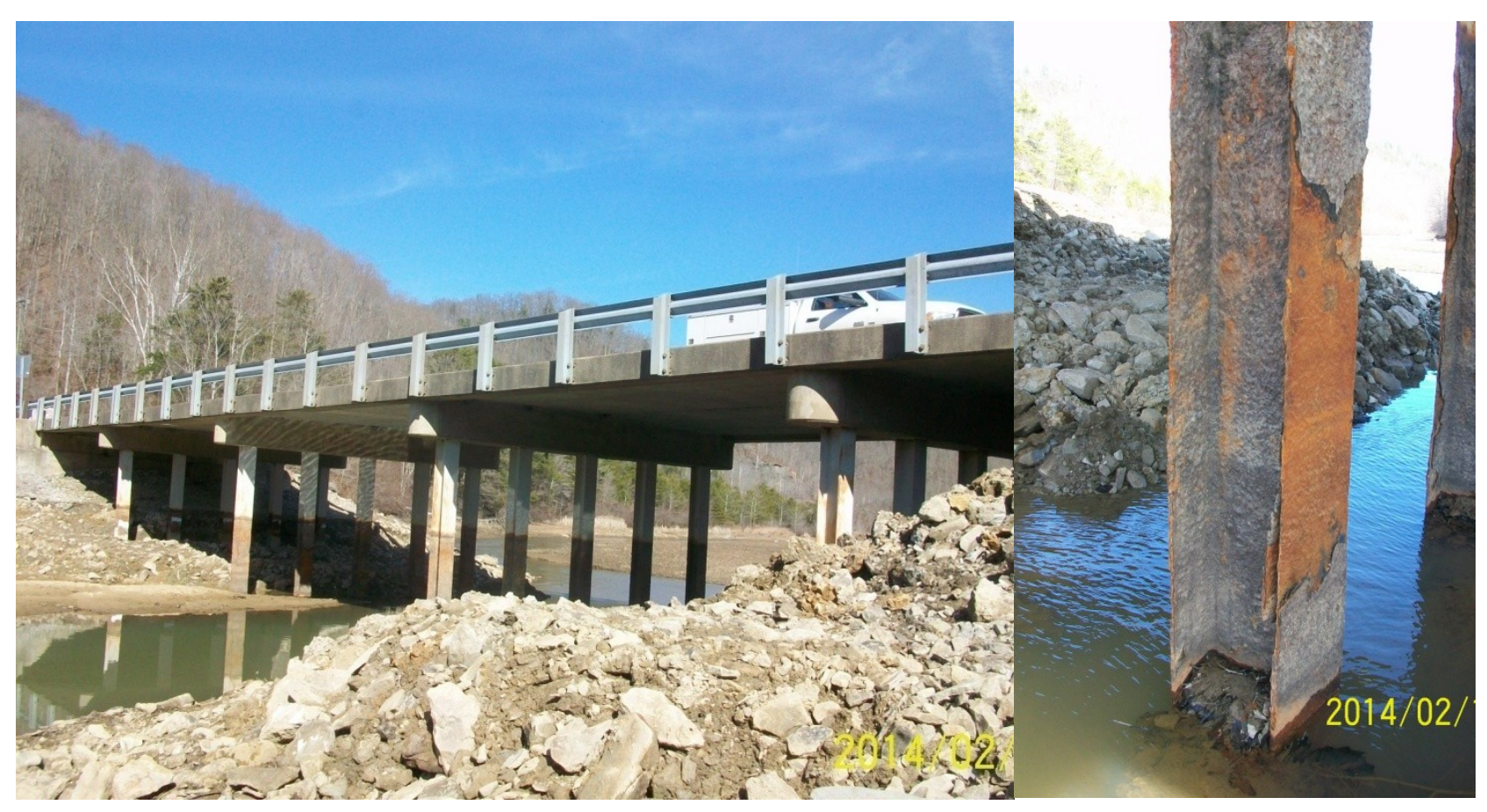

Figure 5.1: East Lynn Lake Bridge (1969) with severely corroded steel structural members, Cove Creek, WV (left); close-up of corroded steel pile (right)

\subsection{Initial Lab Testing}

Before starting the actual field rehabilitation of steel piles in East Lynn Bridge, a preliminary lab testing was performed replicating the field model in order to understand the feasibility of the proposed work and to understand how the FRP wrap and concrete together with steel work in transferring force and with what degree. In this evaluation, 6"x12" concrete cylinders were cast using fast-setting concrete in plastic molds. After 24 hours of casting, the plastic molds were not removed and used as shells beneath to further wrap water curable FRP system "Aquawrap" around them. Concrete sections were cast (Figure 5.2 and Figure 5.3) with and without hot rolled steel section (S4x7.7) to represent H-pile sections in the field (Lake Lynn Bridge). These steel sections were drilled with 1" and 1.5" diameter holes in the web of S4x 7.7 steel section to approximately simulate steel loss due to corrosion (Figure 5.8). 

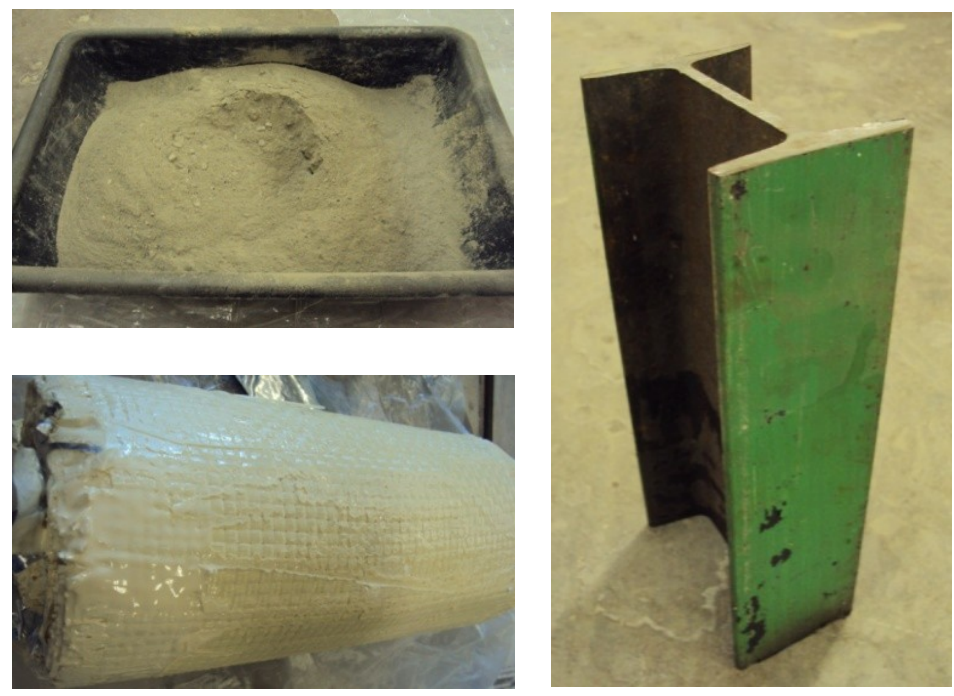

Figure 5.2: Fast-setting concrete, Aquawrap, and steel section S4x7.7
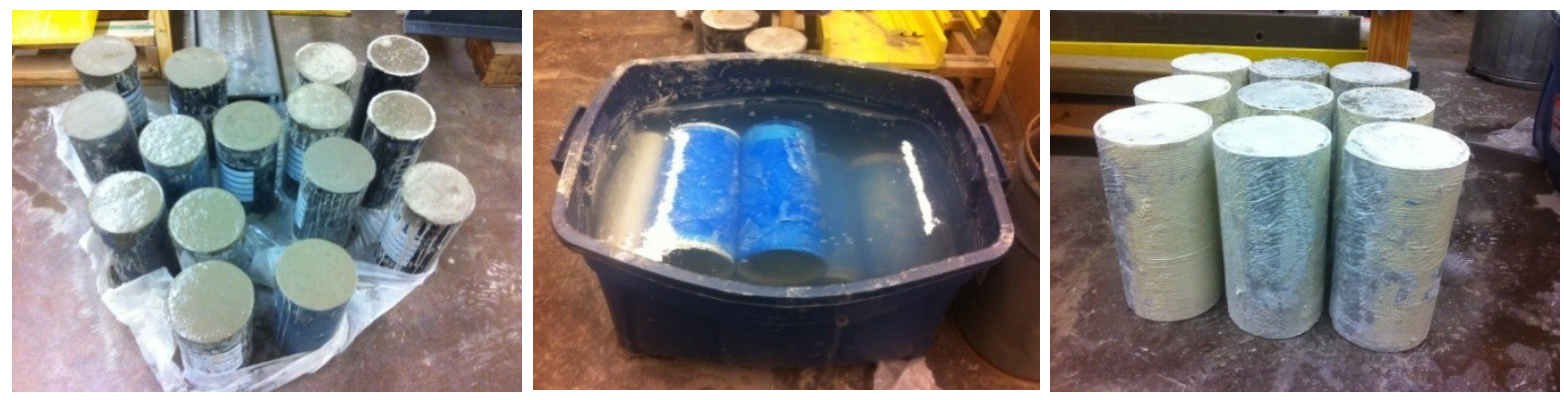

Figure 5.3: Cast concrete cylinders and wrapped concrete cylinders

\subsubsection{Strength of Wrapped Cylinders without Steel Columns}

The unconfined compressive strength of 6"x12" concrete cylinder was found to be 2868 psi $(81,087 \mathrm{lbs})$, whereas the average confined compressive stress with 1 and 2 layers of G-05 Aquawrap was 3063 psi and 3962 psi (86,589 lbs and 112017) (Table 5-1). Thus, the average compressive stress increase was 1094 psi (38.14\%) for 2 layers or 547 psi/per layer (19.07\%). However, strength increase due to single wrap was only $6.78 \%$. Lower effectiveness of single wrap is attributed to lower bond between plastic cylinder shell and wrap and also buckling effects. The stress vs. strain plot for concrete cylinders wrapped with 2 layers of Aquawrap is shown in Figure 5.4. 
Table 5-1: Strength of 6"x12" non-wrapped/wrapped concrete cylinders

\begin{tabular}{|c|c|c|c|c|}
\hline $\begin{array}{c}\text { No. of wrap } \\
\text { layers }\end{array}$ & \multicolumn{2}{|c|}{$\begin{array}{c}\text { Max. axial strength } \\
\text { (lbf) }\end{array}$} & $\begin{array}{c}\text { Avg. axial stress } \\
\text { (psi) }\end{array}$ & $\begin{array}{c}\text { Avg. \% increase } \\
\text { in strength }\end{array}$ \\
\hline \multirow{3}{*}{ None } & $\begin{array}{c}87055 \\
74686\end{array}$ & $\mathbf{8 1 0 8 7}$ & $\mathbf{2 8 6 8}$ & - \\
& 81520 & & & \\
\hline \multirow{3}{*}{1} & $\begin{array}{c}88884 \\
82594\end{array}$ & 86589 & 3063 & $6.8 \%$ \\
& 88290 & & & \\
\hline & 111390 & & 3962 & $38.14 \%$ \\
\hline
\end{tabular}

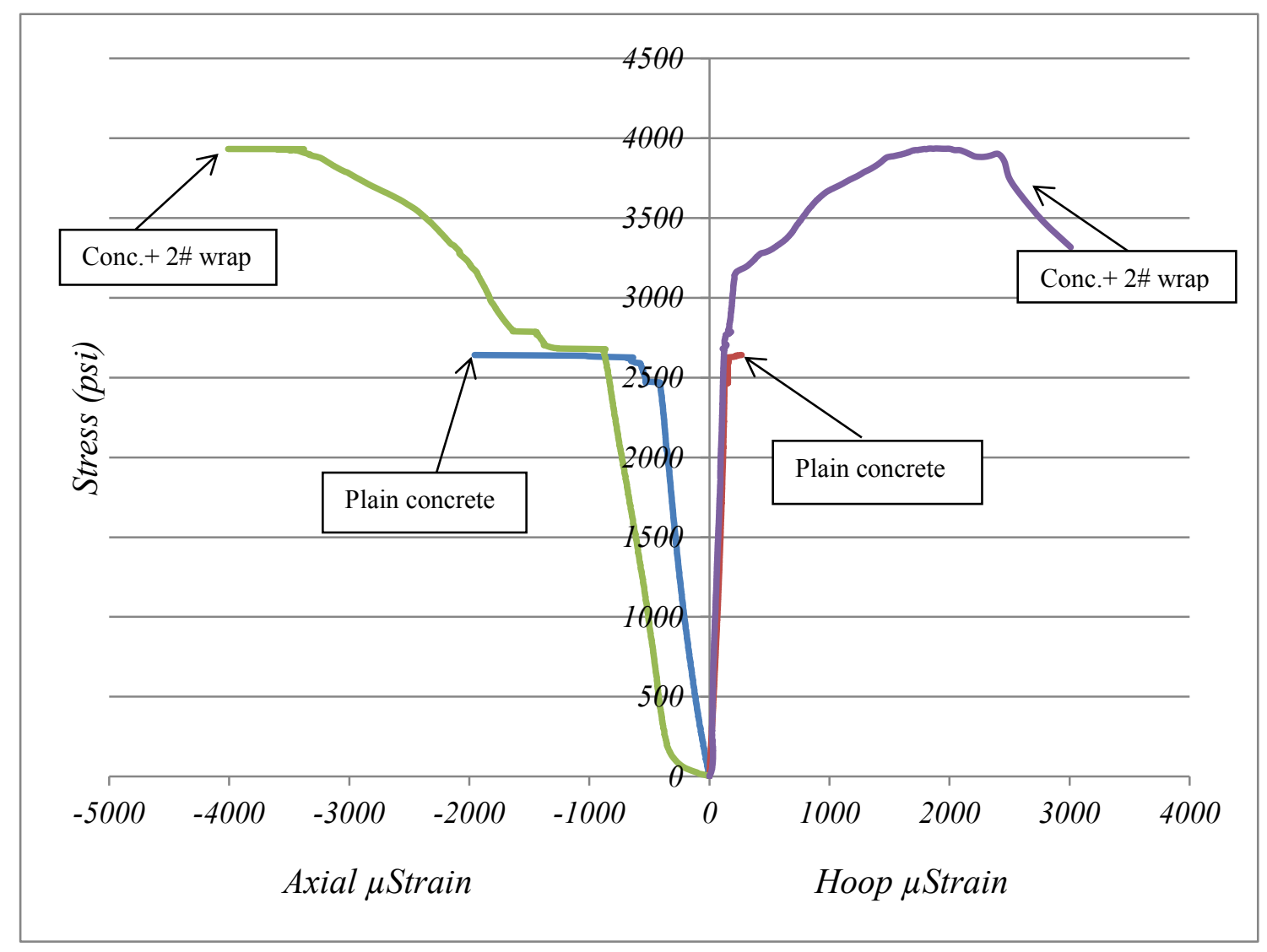

Figure 5.4: Stress vs. strain plot for 6" 12 " concrete cylinder. 


\subsubsection{Strength of Steel Section}

Two 12 inches long steel section S4x7.7 were tested in compression using Instron 1000 HDX. The failure of the steel section on compression test is shown in Figure 5.5. The average failure stress of the steel section was 59.9 ksi (Table 5-2).

Table 5-2: Strength of steel member (S4x7.7) with c/s area of 2.26 in2

\begin{tabular}{|c|c|c|c|c|}
\hline \multirow{2}{*}{ S/n. } & \multicolumn{2}{|c|}{$\begin{array}{r}\text { Max. axial strength to } \\
\text { failure (lbf) }\end{array}$} & \multicolumn{2}{|c|}{$\begin{array}{c}\text { Failure stress } \\
\text { (ksi) }\end{array}$} \\
\hline 1. & 128060 & \multirow{2}{*}{135360} & 56.7 & \multirow{2}{*}{59.9} \\
\cline { 1 - 1 } 2. & 142660 & & 63.1 & \\
\hline
\end{tabular}
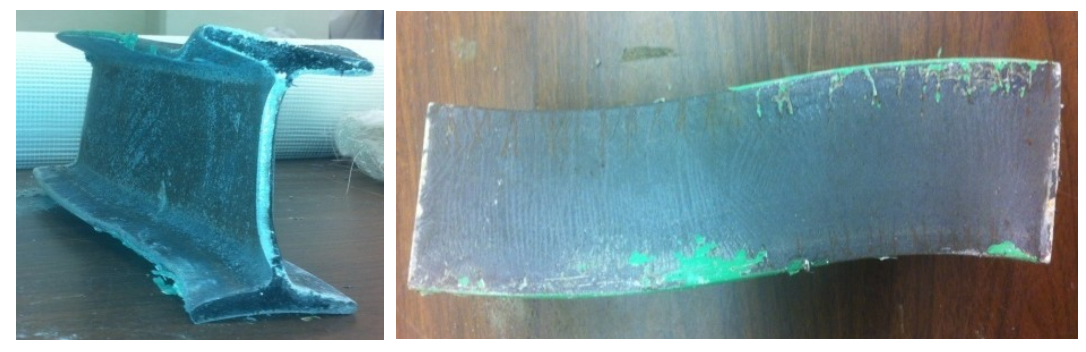

Figure 5.5: Failure of steel member in compression

\subsubsection{Strength of Wrapped Cylinders with Steel Columns}

Following the testing of cylinders with concrete having single and double wraps, next set of casting was carried out using steel sections to represent H-piles. S4x7.7 steel section was cut into $11 \frac{7}{8}$ in. length and was cast together with the concrete cylinder of 12 inches height such that the steel member was just underneath the concrete. After 24 hours of cure, these cylinders were wrapped with 2 layers of Aquawrap fabric. Compressive tests on these cylinders showed an average axial strength of 2.64 ksi (124.2 kips) (Table 5-3). Lower compressive strength of the concrete section with steel (2.64 ksi vs. $3.96 \mathrm{ksi})$ is because of the concrete crushing/wrap rupture occurring first and lack of force transfer between concrete and steel, which was evident 
by the failure along the junction between concrete and steel. In actual field conditions, load is pre-existing on steel sections and for the concrete section to carry live loads, there is a need for effective shear transfer mechanisms.

The load vs. strain plot of concrete cylinder with steel section and 2 layers of Aquawrap is shown in Figure 5.6. To improve compositeness and load transfer between concrete, steel and wraps, steel section was welded with bars and angle sections as discussed in section 4 .

Table 5-3: Strength of concrete cylinders with steel S4x7.7 inside and 2 layers of G-05 Aquawrap outside

\begin{tabular}{|c|c|}
\hline $\begin{array}{c}\text { Avg. axial strength } \\
(\mathrm{lbf})\end{array}$ & $\begin{array}{c}\text { Avg. axial stress (psi) } \\
\text { (converting } A_{s} \text { into total concrete area) }\end{array}$ \\
\hline 124,210 & 2643 \\
\hline
\end{tabular}

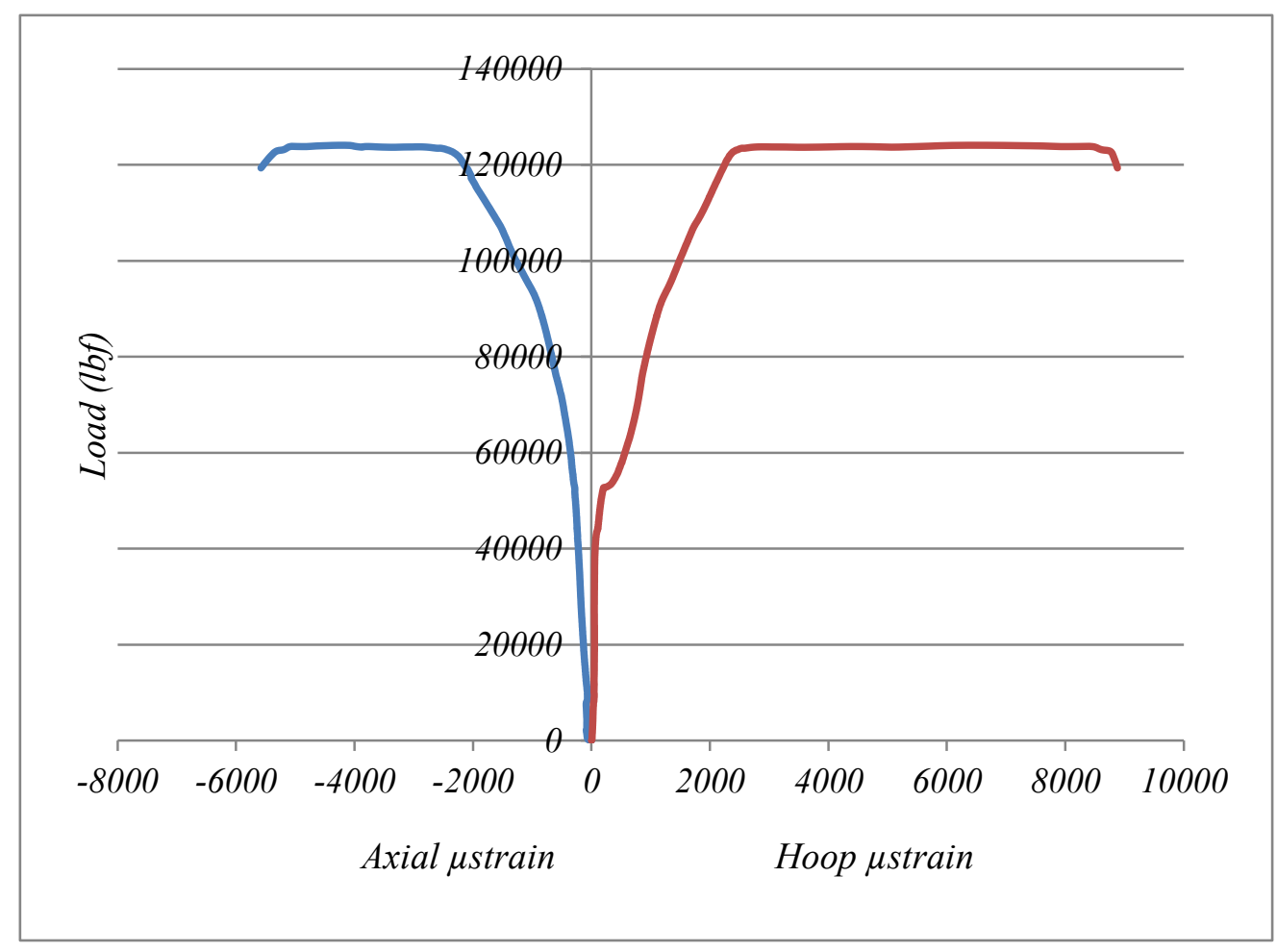

Figure 5.6: Load vs. strain plot for concrete cylinder with steel inside and 2 layers of Aquawrap 

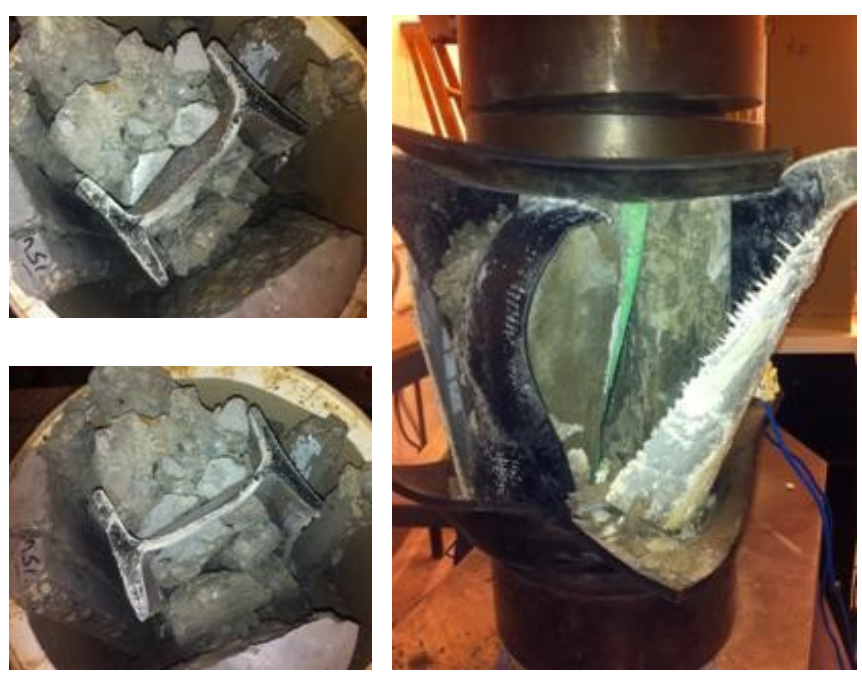

Figure 5.7: Failure of concrete cylinders with steel member and 2 layers of wrap

\subsubsection{Strength of Wrapped Cylinders with Steel Columns having Shear Transfer Bars/Angle Plates}

To enhance composite behavior and transfer of forces between concrete, steel, and FRP, the next set of casting included steel sections with shear transferring bars and plates. Height of the steel section was $12 \frac{1}{8}$ in as opposed to $11 \frac{7}{8}$ in. Increasing the steel section height will transfer the force to steel section first similar to the field condition as opposed to concrete section carrying the load first as in section 3 , where the height of the section was $11 \frac{7}{8} \mathrm{in}$. Steel section with height $12 \frac{1}{8}$ in was modified in four different ways with welded steel rebar (1/2" dia) and angles (1/4" thick) before casting (Figure 5.8 and Table 5-4). Following casting, the cylinders were wrapped with 2 layers of Aquawrap. The compressive load capacities as well as the load vs. strain plots for different variations are shown in Table 5-5and Figure 5.9, respectively. 

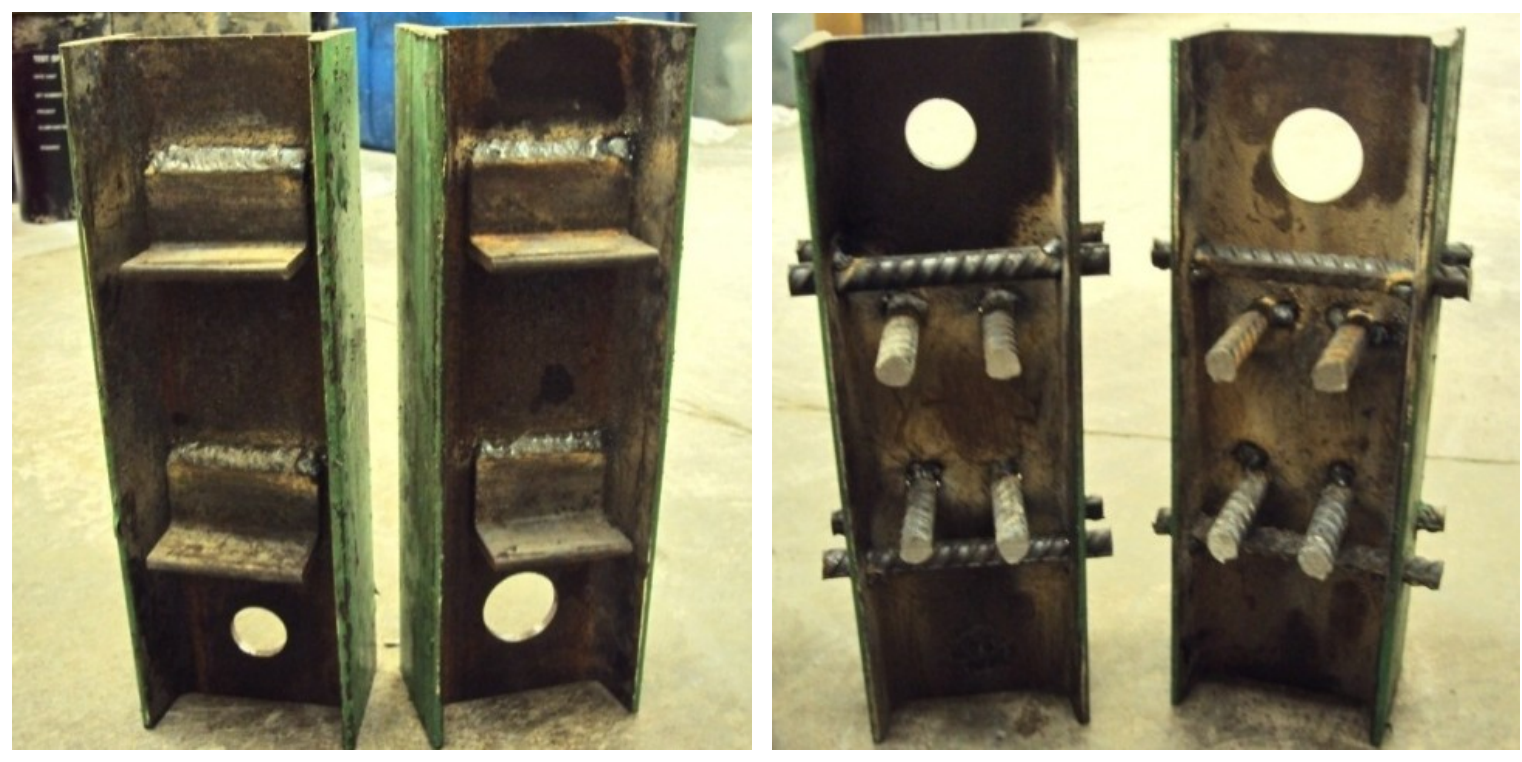

Figure 5.8: Different variations on steel member (V1, V2, V3, and V4) (left to right)

Table 5-4: Different variations on steel member and their corresponding load to failure

\begin{tabular}{|c|l|}
\hline Variations & \multicolumn{1}{|c|}{ Description } \\
\hline V1 & 1 in. hole, rebar, and 2 layers of Aquawrap \\
\hline V2 & 1.5 in. hole, rebar, and 2 layers of Aquawrap \\
\hline V3 & 1 in. hole, angles, and 2 layers of Aquawrap \\
\hline V4 & 1.5 in. hole, angles, and 2 layers of Aquawrap \\
\hline
\end{tabular}




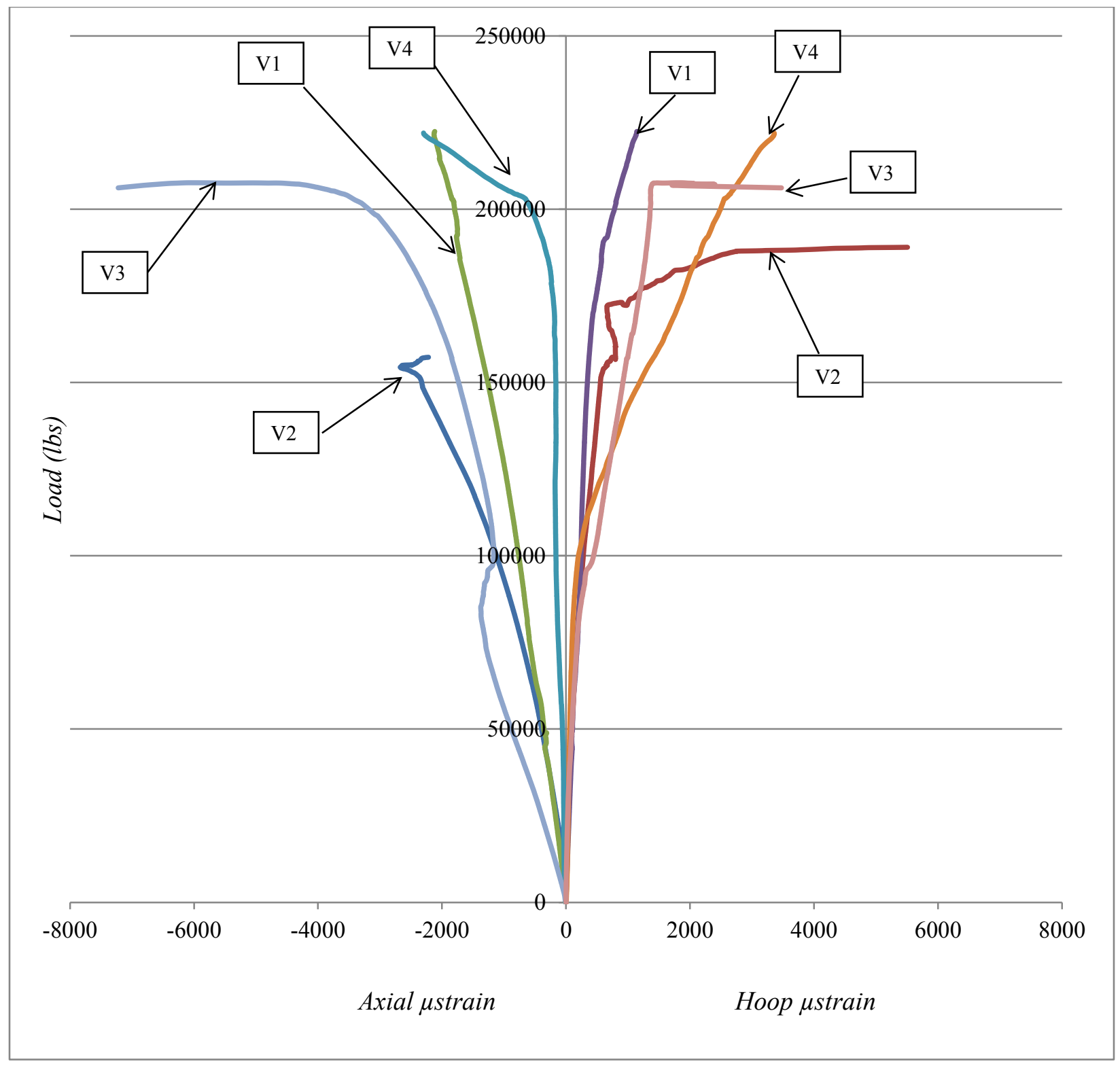

Figure 5.9: Load vs. strain plots for different variations on steel members

\subsubsection{Summary}

The Table 5-5 summarizes the load capacity of steel section, concrete cylinders and wrapped concrete cylinders with and without steel section. The failure of the wrapped concrete cylinders with steel section inside is shown in Figure 5.10. 
Table 5-5: Summary of different tests

\begin{tabular}{|l|c|c|}
\hline \multicolumn{1}{|c|}{ Variations } & $\begin{array}{c}\text { Avg. max. load to } \\
\text { failure (kips) }\end{array}$ & $\begin{array}{c}\text { Avg. max. stress (psi) } \\
\text { (converting } A_{s} \text { into total } \\
\text { concrete area) }\end{array}$ \\
\hline Plain concrete (Table 5-1) & 81.1 & 2868 \\
\hline Concrete + 2\# wrap (Table 5-1) & 112.02 & 3962 \\
\hline Steel section (Table 5-2) & 135.36 & 59,900 \\
\hline $\begin{array}{l}\text { Concrete + steel + 2\# wrap } \\
\text { (Table 5-3) }\end{array}$ & 124.21 & 2643 \\
\hline V1 (Table 5-4) & 216.55 & 4607 \\
\hline V2 (Table 5-4) & 196.05 & 4171 \\
\hline V3 (Table 5-4) & 207.7 & 4419 \\
\hline V4 (Table 5-4) & $>222$ & $>4723$ \\
\hline
\end{tabular}

Note: V4 did not fail as max. load capacity of the Instron machine was reached.

Note:

1. Calculation of equivalent concrete area of steel section:

According to ACI code, the modulus of elasticity of concrete,

$$
E_{c}=57000 \overline{f_{c}^{\prime}}=57000 \overline{3000}=3122 k s i
$$

And, the modulus of elasticity of steel, $E_{s}=29 \mathrm{msi}$

Modular ratio (n) $=\frac{E_{S}}{E_{c}}=9.289$, and equivalent concrete area of steel section $=n A_{S}$

Thus, the total c/s area of the concrete cylinder $=A_{c}+n A_{s}$

$$
\begin{aligned}
& =\left[(A-2.26)+n\left(A_{s}\right)\right] \mathrm{in}^{2} \\
& =[28.274-2.26+9.289 * 2.26] \mathrm{in}^{2} \\
& =47 \mathrm{in}^{2}
\end{aligned}
$$


2. Theoretical maximum load with full compositeness $=(112.02-2.26 * 2.868)+$ $135.36=241$ kips (buckling and contribution of holes are neglected).

3. Maximum degree of compositeness was $92.1 \%$ (222 kips / $241 \mathrm{kips})$ and the degree of compositeness can be further enhanced with additional shear transfer mechanisms such as bars and plates.

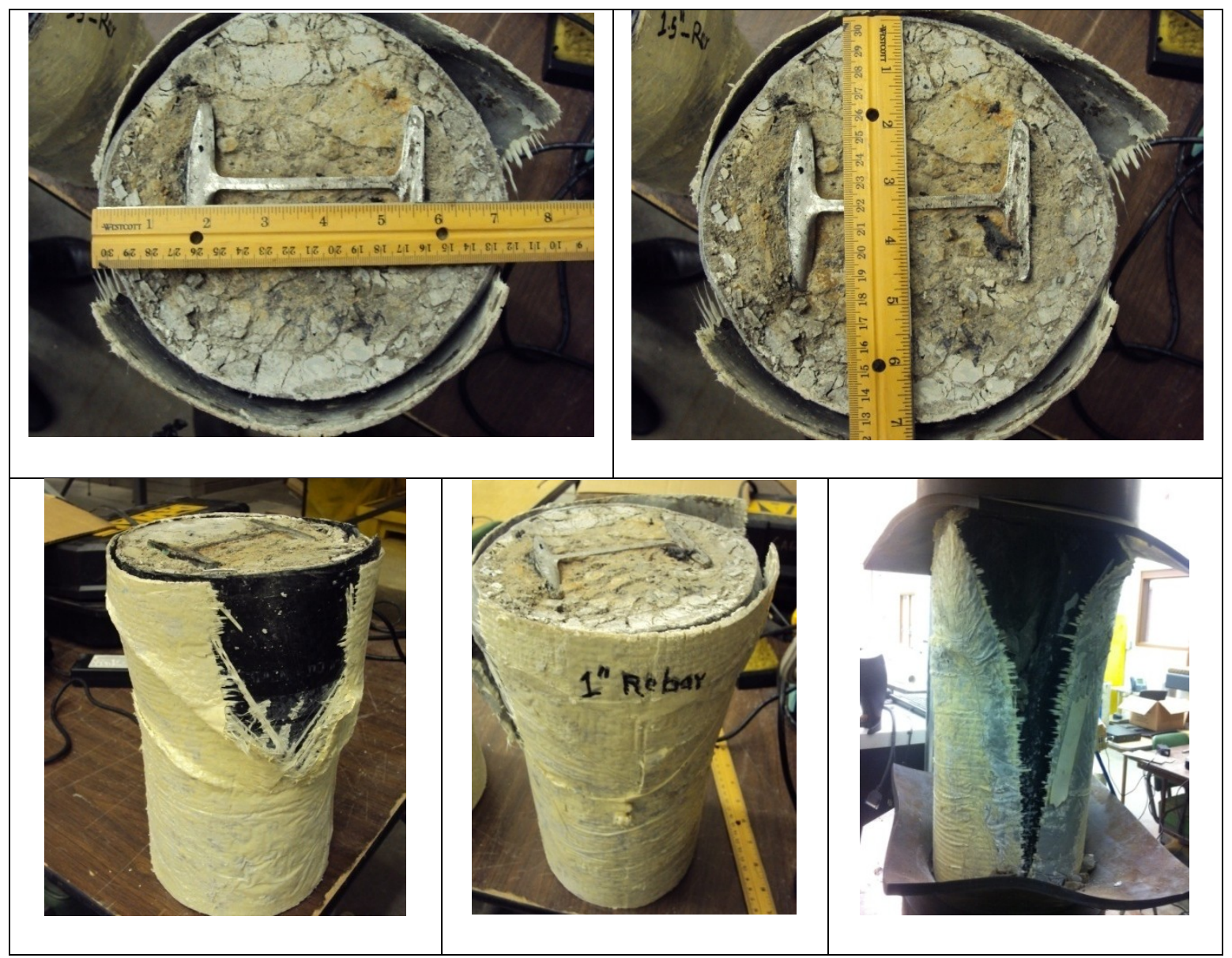

Figure 5.10: Failure of the wrapped concrete cylinders with steel inside 


\subsection{Conclusions of the lab testing}

Following conclusions are drawn from this study.

1. Concrete and FRP wrap contributed significantly to the load capacity of the piles.

2. Minimum of two layers of wrapping are recommended to avoid bond related failure modes and local buckling.

3. Without shear force transfer mechanism, strength enhancement will be minimum and the force transfer between concrete and steel is not effective. They act individually without force transfer mechanism.

4. Maximum degree of compositeness exceeded $92 \%$ (222 kips /241 kips) and the degree of compositeness can be further enhanced with additional shear transfer mechanisms such as bars and plates.

\subsection{Field Rehabilitation}

This work will consist of reducing and preventing corrosion of H-piles including improvement of their load capacity through FRP wrapping. Steel piles with section loss and deterioration will be cleaned of loose debris and corrosion by-products through pressure washing using a 5000 psi pump. Necessary corrosion treatment/monitoring schemes are being implemented prior to rehabilitation. Cofferdam protection will be provided around the piles and wrapping will extend to a depth of up to a minimum of half feet or beyond the corrosion zone.

Circular FRP shells of 20" diameter with tongue and groove joints will be placed around the H-piles up to required heights. Additional fastening/bonding schemes will be used on need basis to hold the shell in place around the piles and two layer circumferential GFRP wrapping 
over the shell. Bottom part of the FRP shell will be grouted with up to 9" height of epoxy grout. Rest of the shell will be filled with concrete and cement grout, which will be pumped through the top port on the FRP shell.

Following field installation, field monitoring will be carried out in terms of NonDestructive Testing (infrared thermography and electronic tap hammer) for bond integrity evaluations. In addition, corrosion rate evaluation, thermal strains, deformations under loading, humidity levels, and others will be monitored. Calibrated truck loads will be used for load tests prior to and after rehabilitation. The following sections give the process involved in the rehabilitation in details.

Step 1: The first work that was done in the field was channelizing the water from the portion between the two middle pier bents and removal of the mud around the piles (about 1-3 feet) to the level where corrosion was seen. The steel piles below the mud line showed minimum corrosion or absence of corrosion due to lack of oxygen supply. Steel piles with section loss were pressure washed and to remove loose debris and corrosion by-products using 5000 psi water pump (Figure 5.11). The scaffolding was arranged around the pier bents such that there was an access to walk around the piles as well as to reach to the top of the pile (Figure 5.12). Six number of shear transferring rods were attached to the web and flanges of each pile 1-2 feet above the mud line (Figure 5.12). 


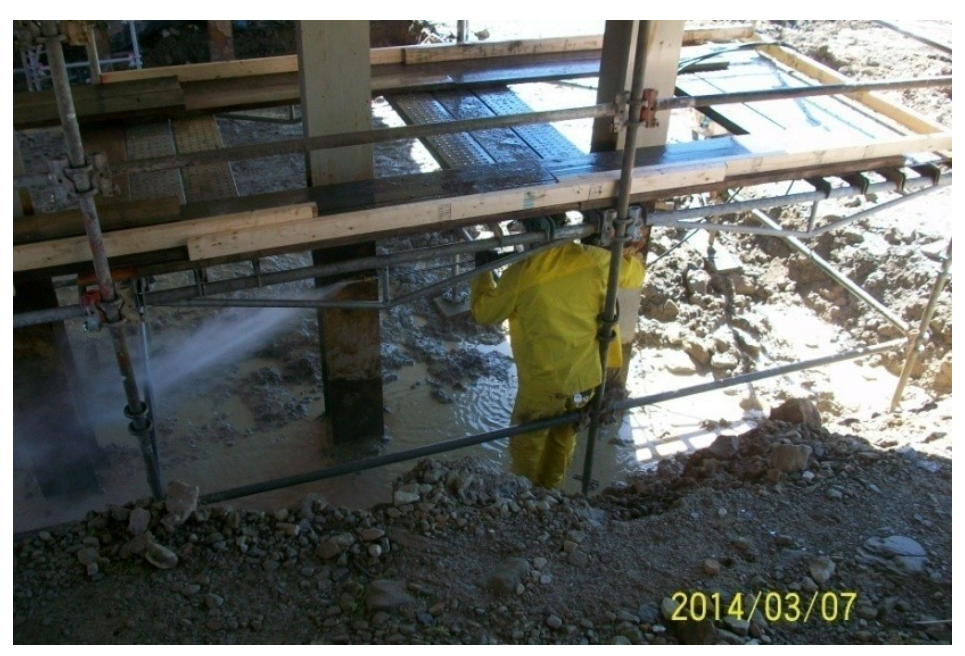

Figure 5.11: Pressure washing of piles
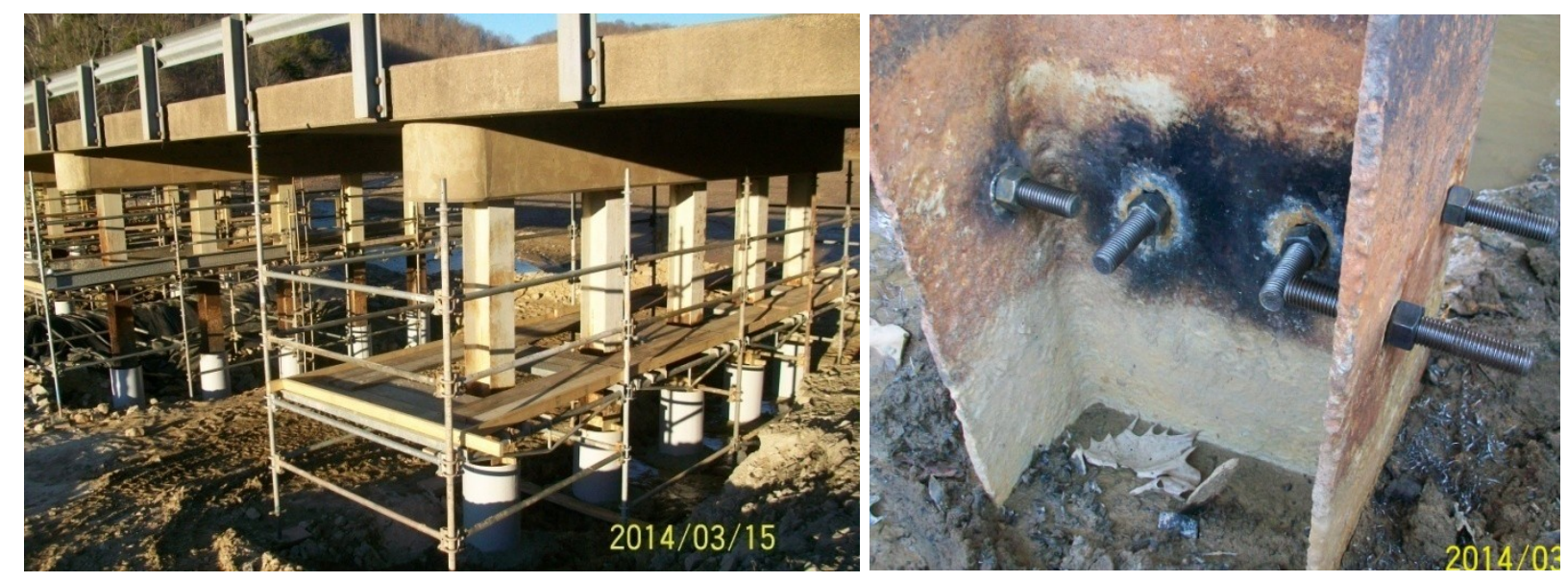

Figure 5.12: Scaffolding placed around the piles (left) and shear transferring rods placed (right)

Step 2: After the scaffolding was placed around the piles, 3' high circular FRP shells/jackets of 20" diameter with tongue and groove joints (manufactured in three different lengths of 3', 4' and 6') were initially placed around all the H-piles resting on the mud line with about 2 inches into the mud. The epoxy glue was used in the groove for proper bonding with the tongue and bolts were placed along the length of the joint at 8 " center to center (Figure 5.13). 

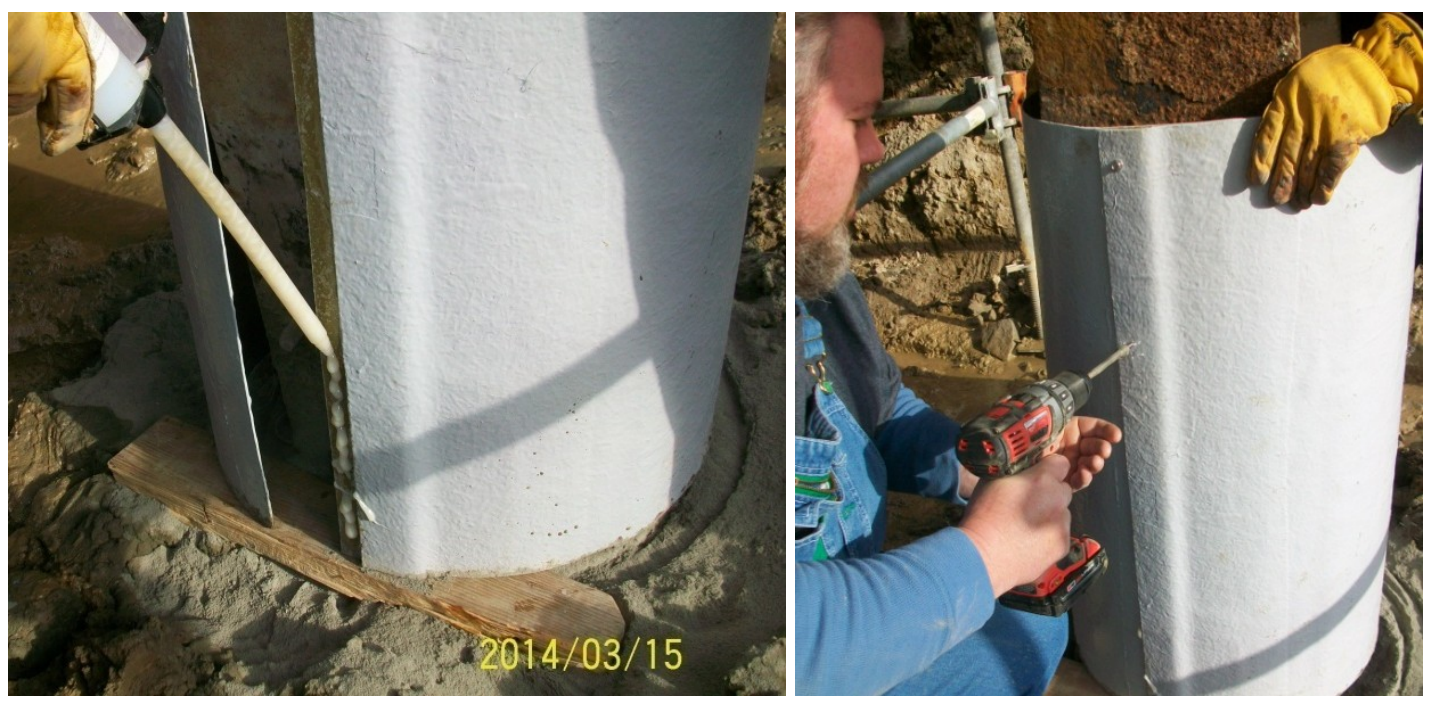

Figure 5.13: Placement of FRP jackets around the pile and gluing the joint

Step 3: After the placement of FRP shells, "SIMPSON-Strong-Tie" polymer concrete was poured into the shells up to a depth of 8 " to 12 ". Polymer concrete/grout comes with 2 parts of resin and the filler materials which were mixed together in the manufacturer specified ratio (Figure 5.14). After curing the grout for 3 days, other FRP shells were again attached on top of 3' FRP shells already placed around the H-piles. The shells were placed all around the piles to the total height until the pier cap was reached. The joint regions were glued and further bolted together (Figure 5.14). On top part of all shells, a square (6" x 6") hole was cut for pouring the self-consolidating concrete into the shells. A wooden box with a slope inward was fixed into the hole for pouring the concrete through a concrete pump discharge hose (Figure 5.15). 


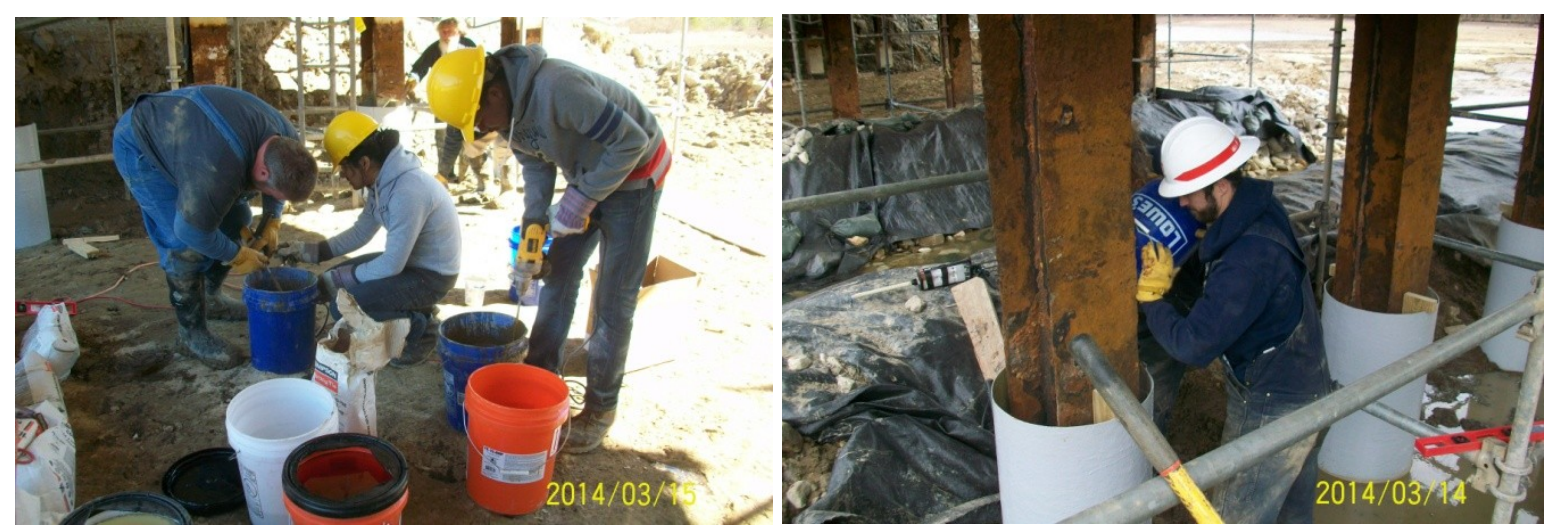

Figure 5.14: Mixing of polymer grout and placing them into the FRP shell around the pile
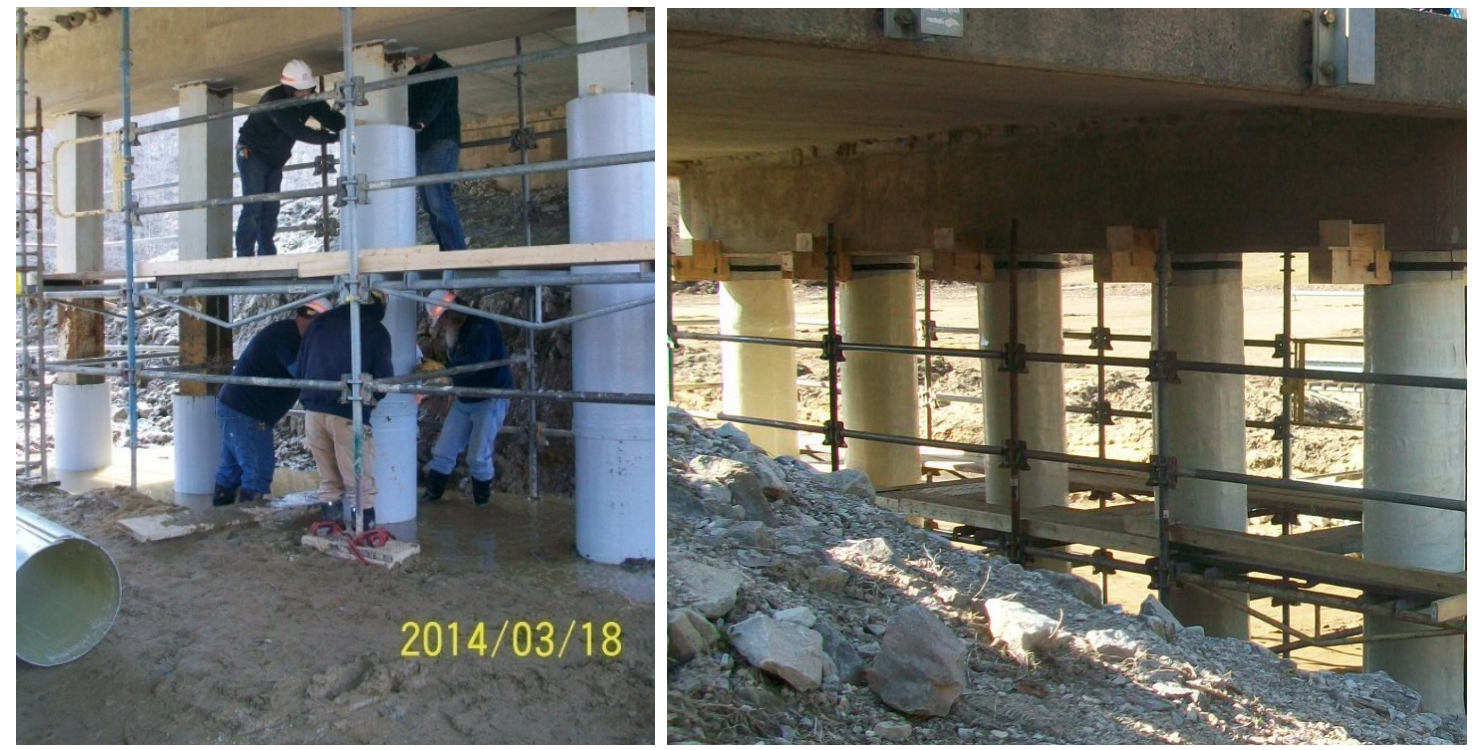

Figure 5.15: Stacking of FRP shells and placing the wooden box for pouring concrete

Step 4: Once FRP shells were fixed and grouted around all piles, they were wrapped with 2 layers of Aquawrap. After mixing two parts of BP-1 primer, it was applied over FRP jackets with a hand roller. Water was continuously sprayed over the FRP jackets once the primer was applied. Then, Aquawrap pouch was opened and wrapping was started from the top of the pile, spiraling it downwards with an overlap of around 2-3 inches. After reaching the base, the wrap was further spiraled upwards reaching to the top. Spraying of water was done simultaneously with the wrapping process as Aquawrap needs water to cure. After, the wrapping was completed, 
two layers of stricture banding were placed all around the wrap and finally the banding was perforated using perforator for curing process (Figure 5.16). The detailed procedure for wrapping Aquawrap is presented in Chapter 3 of this thesis.

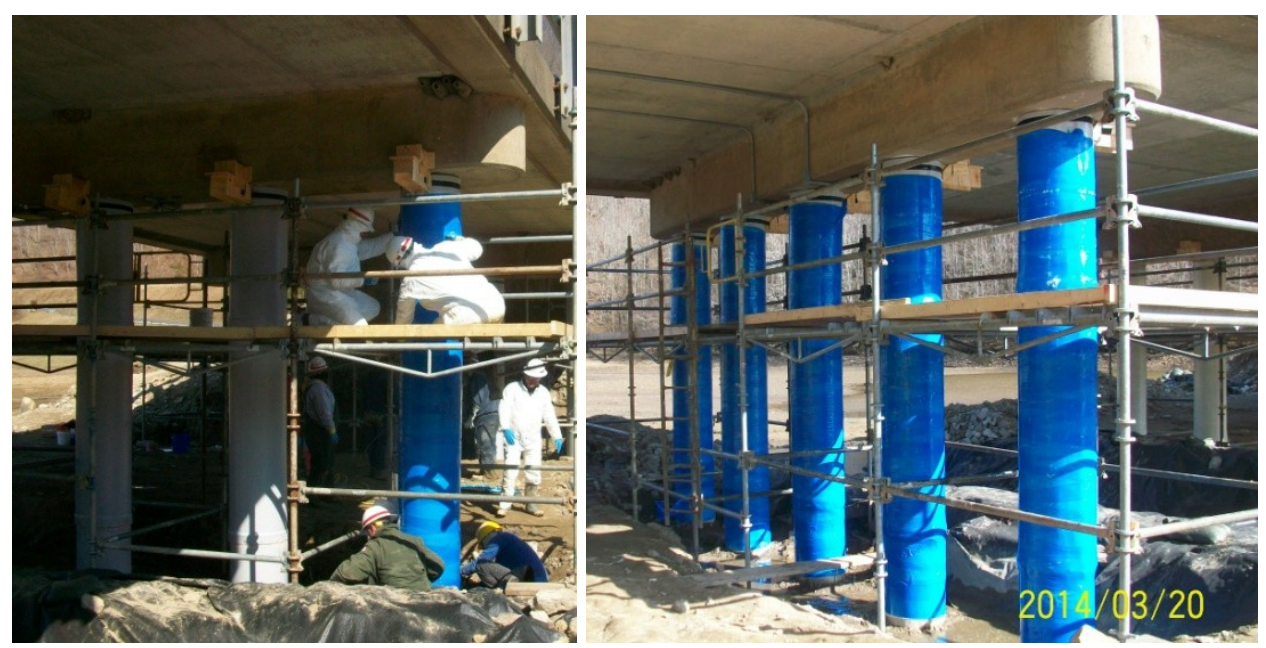

Figure 5.16: Wrapping and stricture banding of the piles

Step 5: Finally, after the wrap was cured for a week, self-consolidating concrete was poured into the wrapped FRP shells (Figure 5.17). After the placement of concrete, the wooden box arrangements were removed and the top portion of the bridge piles was wrapped with 2 layers of Aquawrap (Figure 5.17). The final view of the bridge is shown in Figure 5.18. The final step was to paint the bridge for aesthetic and UV protection requirements.
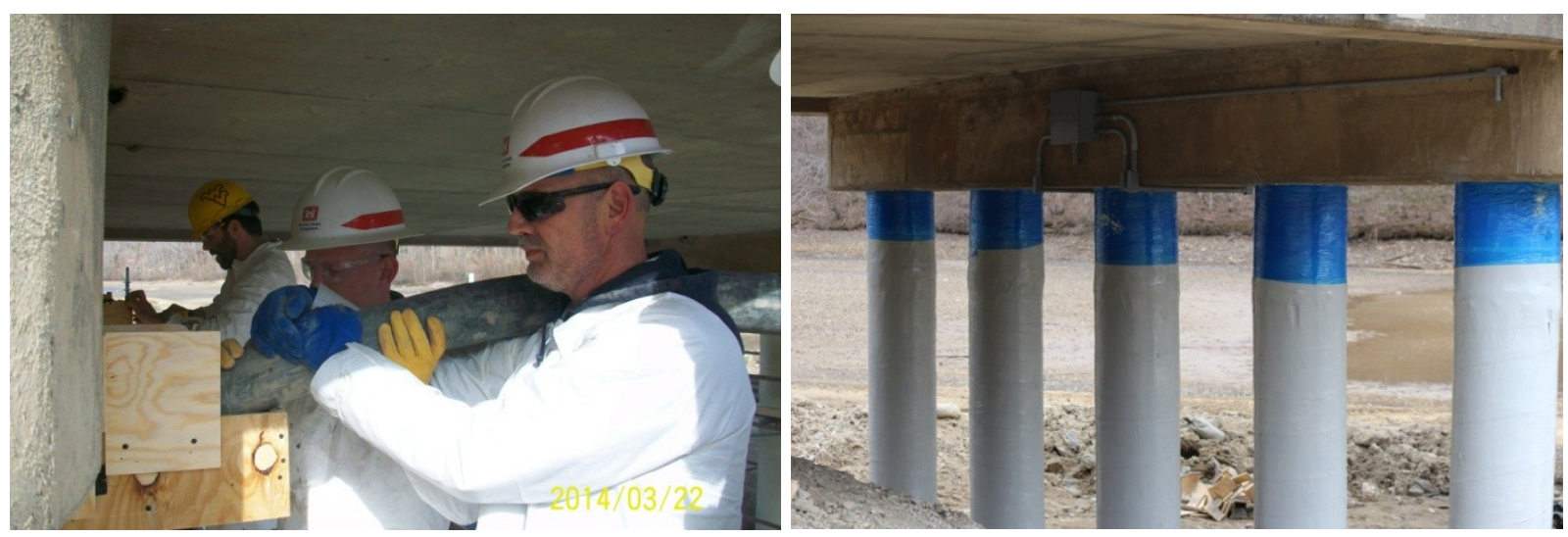

Figure 5.17: Pouring of concrete and stricture banding on the top portion of the piles 


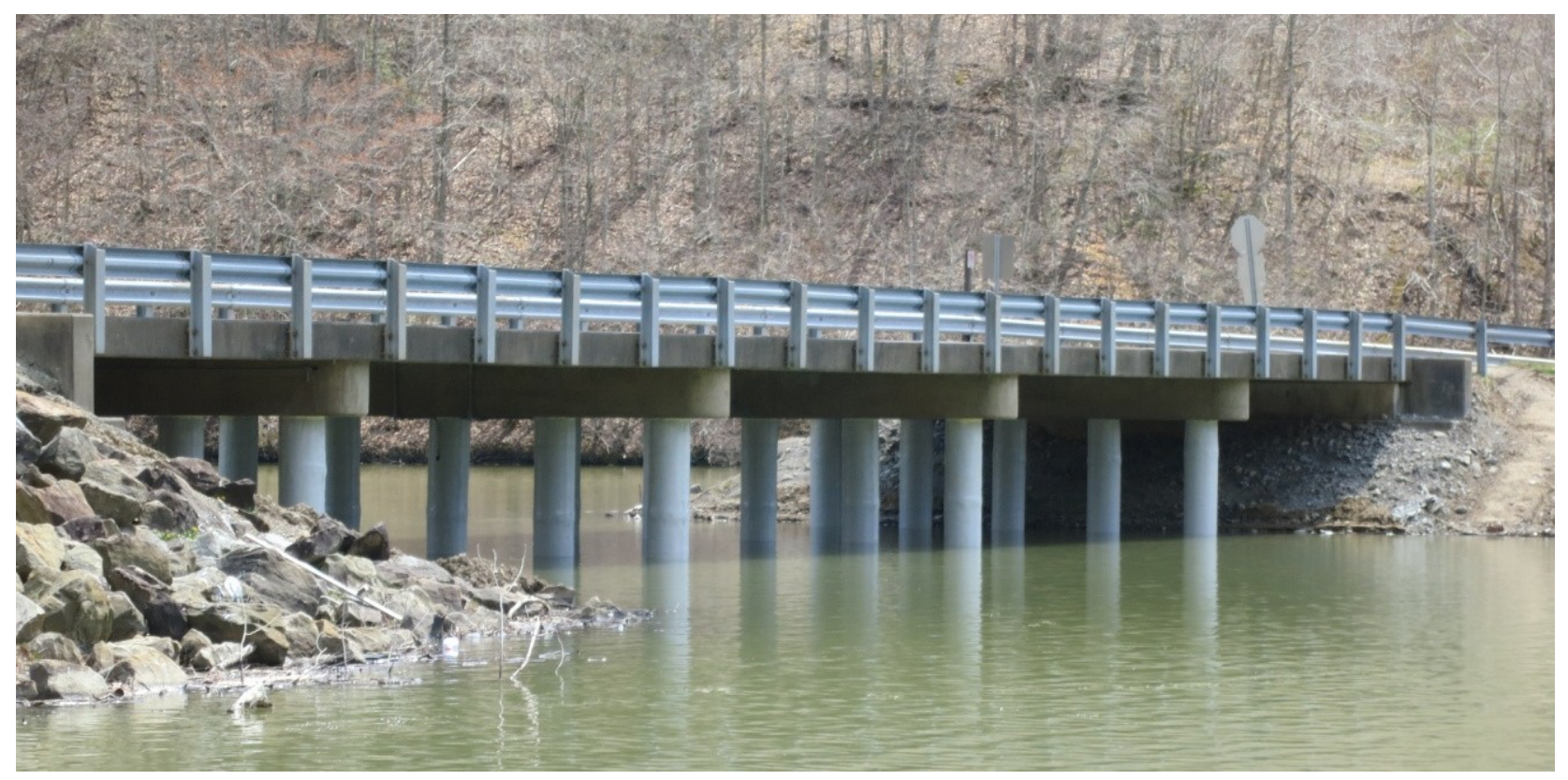

Figure 5.18: Final view of the bridge after rehabilitation

\subsection{Strength calculations for rehab of steel H-piles/columns}

The strip specimens of 12 " long were cut from FRP shell of thickness 0.125 " in both longitudinal and circumferential direction (Figure 5.19 and 5.20) and also from 2 attached layers of Aquawrap with thickness $(2 * 0.0285=0.057 ")$. The tensile strengths are shown in Table 5-6 and 5-7.

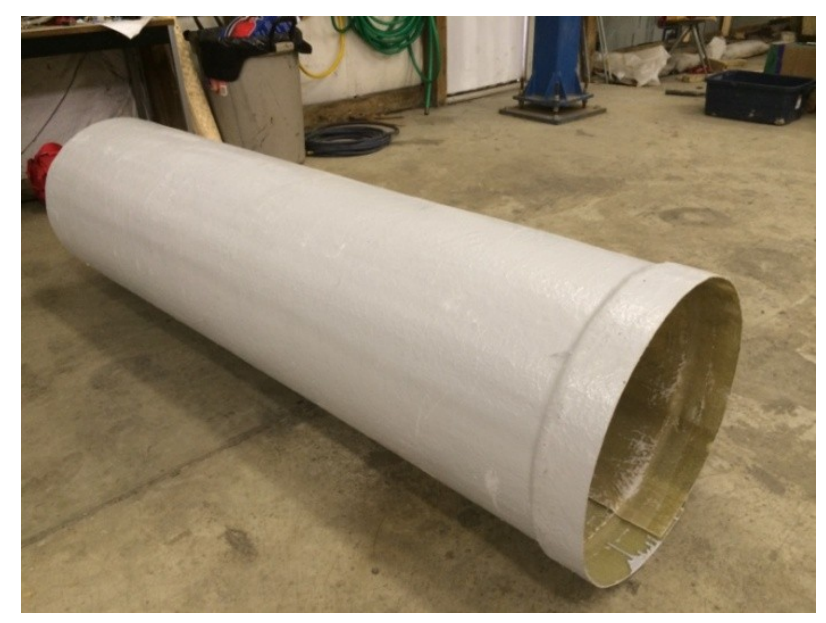

Figure 5.19: FRP shell/jacket 

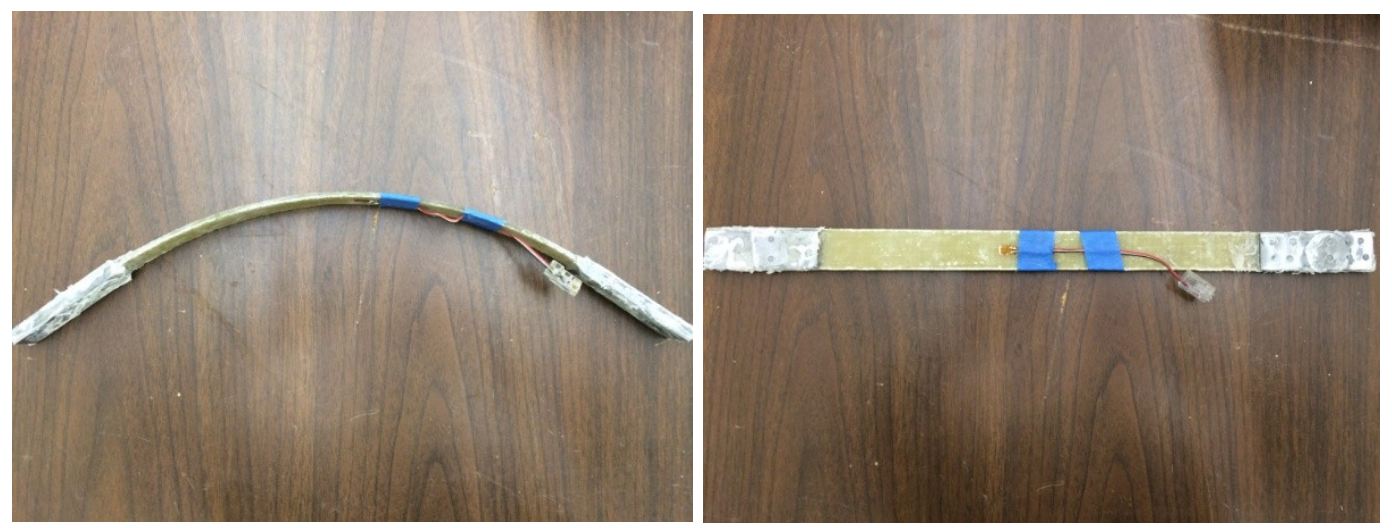

Figure 5.20: FRP strip cut from circumferential direction (left) $\&$ from longitudinal direction (right)

Table 5-6: Tensile stress of 2\# of Aquawrap strip

\begin{tabular}{|c|c|c|}
\hline \# FRP layers & $\begin{array}{c}\text { Average max. load } \\
(\mathrm{lbs})\end{array}$ & $\begin{array}{c}\text { Tensile stress } \\
(\mathrm{ksi})\end{array}$ \\
\hline 2 & 2323 & 40.7 \\
\hline
\end{tabular}

Table 5-7: Tensile stress of strips cut from FRP shell

\begin{tabular}{|c|c|c|c|c|c|}
\hline S/no & Strip location & $\begin{array}{c}\text { Max.load@ } \\
\text { failure (lbs) }\end{array}$ & $\begin{array}{l}\text { Avg. load } \\
\text { (lbs) }\end{array}$ & $\begin{array}{c}\text { Tensile stress } \\
(\mathrm{ksi})\end{array}$ & $\begin{array}{c}\text { Max.strain@ failure } \\
\text { (lbs) }\end{array}$ \\
\hline 1. & Circumferential & 2368 & \multirow{3}{*}{2331} & \multirow{3}{*}{18.7} & 13710 \\
\hline 2. & $"$ & 2426 & & & - \\
\hline 3. & $"$ & 2200 & & & - \\
\hline 4. & Longitudinal & 2253 & \multirow{2}{*}{2404} & \multirow{2}{*}{19.2} & - \\
\hline 5. & $"$ & 2555 & & & 15362 \\
\hline
\end{tabular}

Also, the concrete cylinders of 4" $\mathrm{x} 8$ " cast at the site with self-consolidated concrete were tested in compression in the lab at 14 and 28 days and the results are shown in Table 5-8.

Table 5-8: Compressive strength of 4" x 8" Self-Consolidated Concrete (SCC) cylinders

\begin{tabular}{|c|c|c|c|c|}
\hline S/no & Strength & (psi) & Stren & \\
\hline 1. & 2760 & \multirow[b]{3}{*}{2801} & 3100 & \multirow{3}{*}{3050} \\
\hline 2. & 2800 & & 3103 & \\
\hline 3. & 2844 & & 2948 & \\
\hline
\end{tabular}

Figure 5.21 shows the rehabilitated pile geometry of Ease Lynn Lake Bridge. The computation of strength of the rehabilitated bridge piles is shown in details below. 


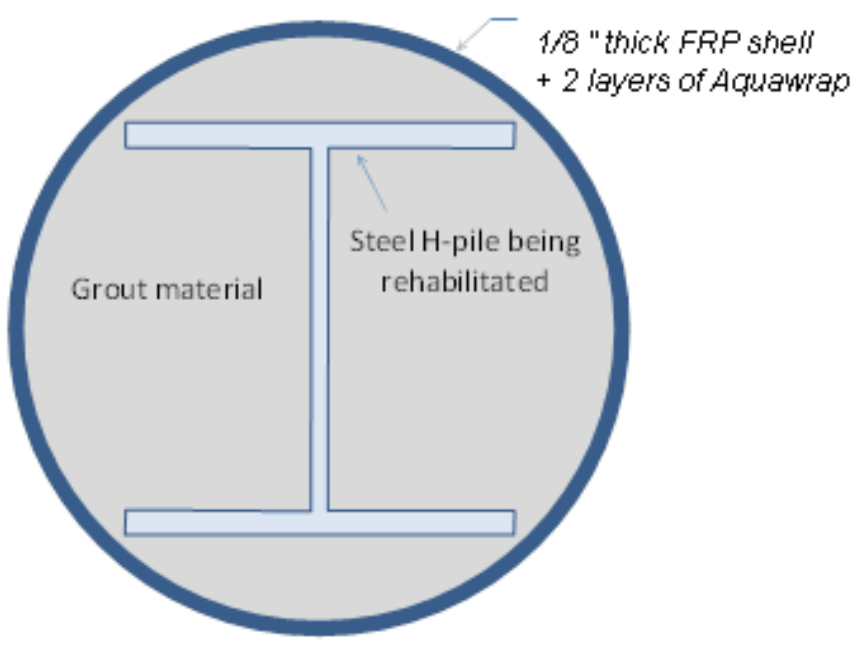

Figure 5.21: Rehabilitated pile geometry

\section{Calculation of Concrete area}

Outer diameter $=20 "$

GFRP shell and wrap thickness $=0.182^{\prime \prime} \sim 0.2^{\prime \prime}$

Diameter of the concrete $=20-2 * 0.2=19.6 \mathrm{in}^{2}$

Pristine Steel $H-$ pile $\left(\mathbf{1 2}\right.$ BP 53) $c / s$ area $=15.5 \mathrm{in}^{2}$

For damaged steel $\mathrm{H}-$ pile, area of cross - section $=7.4 \mathrm{in}^{2}$

Concrete area $=\pi \frac{d^{2}}{4}-$ steel area

$$
\begin{aligned}
& \left.=\pi \frac{(19.6)^{2}}{4}-7.4 \text { (for damaged steel column }\right) \\
& =301.72-7.4 \\
& =294 \mathrm{in}^{2}
\end{aligned}
$$

Strength improvement due to concrete confinement over unconfined concrete

The calculations of strength improvement due to confinement as per ACI $440.2 \mathrm{R}-08$ is: 


$$
\begin{aligned}
& \frac{f_{c c}^{\prime}=\text { confined concrete strength }}{f_{c o}^{\prime}=\text { unconfined concrete strength }}=1+3.3 \frac{\left(f_{l 1}+f_{l 2}\right)}{f_{c o}^{\prime}} \\
& f_{l}=f_{l 1}+f_{l 2}=\text { total lateral confining pressure } \\
& f_{l 1}=\text { lateral confining pressure by GFRPshell }=\frac{2 E_{f 1} n t_{f 1} \epsilon_{f e}}{D} \\
& f_{l 2}=\text { lateral confining pressure by GFRP wrap }=\frac{2 E_{f 2} n t_{f 2} \epsilon_{f e}}{D} \\
& t_{f 1} \cong \text { thickness of FRP shell/layer }=0.125 " \\
& t_{f 2} \cong \text { thickness of FRP fabric/layer }=0.02875 " \\
& n \cong n u m b e r \text { of } F R P \text { shell/FRP } \\
& D=\text { Diameter of circular section being wrapped } \cong 20 " \\
& \epsilon_{f e}=\text { Effective failure strain of GFRP shell/fabric }=0.55 \quad 15,000 \quad 10^{-6} \\
& =8250 \times 10^{-6} \\
& f_{l 1}=\frac{2 \times 1.0 \times 10^{6} \times 8250 \times 10^{-6} \times 1 \text { layer }(0.125)}{20}=103 \\
& f_{l 2}=\frac{2 \times 2.28 \times 10^{6} \times 8250 \times 10^{-6} \times(2 \text { layers })(0.0285)}{20}=107 \\
& \frac{f_{c c}^{\prime}}{f_{c o}^{\prime}}=1+3.3 \frac{103+107}{3000}=1.23
\end{aligned}
$$

However, we conservatively consider only 1.20 (instead of 1.23) because of size effect of column having a diameter of 20 " and field variations.

The unconfined strength of SCC concrete from testing of cylinders $=3 \mathrm{ksi}$

$$
\therefore f_{c c}^{\prime}=1.2 * f_{c o}^{\prime}=1.2 * 3=3.6 k s i
$$

Total strength of the confined concrete due to FRP $=3.6 \mathrm{ksi} * 294 \mathrm{in}^{2}=1060$ kips Also, the strength of the damaged steel 12 BP 53 section $=36 \mathrm{ksi} * 7.4 \mathrm{in}^{2}=266$ kips Finally, total strength of the rehab. portion of the pile $=1060+266=1326$ kips 
The rehabilitation scheme adopted for East Lynn Lake Bridge has resulted in the failure capacity of 1326 kips for the rehabilitated portion of each column while a pristine steel column (12 BP 53) would carry 558 kips to yield.

\subsection{Conclusions}

1. Concrete cylinders with steel section and wrapped with 2 layers of Aquawrap showed significant increase in load capacity with degree of compositeness being greater than $92 \%$.

2. The rehabilitation scheme resulted in enhancing the column failure load capacity by $\sim 2.5$ times that of yield capacity of 12 BP 53 section. Despite rehabilitation and increased load capacity, the steel columns will only be rated up to their original capacity to yield which is 558 kips per column of $36 \mathrm{ksi}$ steel.

3. Weight limit after successful rehabilitation has been upgraded and increased from 6 tons to 30 tons.

4. The concrete and the FRP composites (jackets and wraps) that encase the corroded steel provide three layers of protection against future corrosion and give superior strength (corrosion probes have been installed in the site for studying the future corrosion rate).

5. The use of FRP composites for rehabilitation provided cost and time advantage (cost is about $2 / 3$ less than conventional construction and time for the completion of the rehabilitation is about 3 weeks) including reduction in future maintenance costs and enhanced durability.

6. The temperature-humidity levels influence the material curing properties and site work progress and require the work-plan to accommodate those variations. 


\section{CHAPTER 6 ABRASION RESISTANT COATINGS}

\subsection{Introduction}

This research is focused on the study of abrasion characteristics as well as comparative abrasion tests on different commercially available abrasion resistant coatings. The purpose of this study is to come up with composite based coating that can withstand hydraulic forces from high velocity water as well as severe cutting, impact and abrasive forces from barges and sand particles flowing along with the water in tainter gates at Heflin Lock \& Dam near Gainesville, Alabama. Conditions of severe abrasion exist at some high-lift dams that cause standard protective coatings on the downstream side of tainter gates to be abraded away in just a few years of service (Figure 6.1). Down-stream side of protective coating system is damaged typically by debris that swirls and bangs against the gate causing severe cutting and abrasion to the coatings applied on those gates.

Generally, US Army Corps of Engineers use vinyl based coatings on such steel gates in order to protect them from corrosion but due to the existence of such abrasive forces, the coating gets abraded easily at a faster rate resulting in expensive repairing efforts. Thus, the focus of this effort was to come up with composite coating system or alternative systems that can withstand the abrasive action of water and other unwanted particles and debris flowing with it. The abrasive wear resistance of ceramic composite coatings is generally high as composites help in achieving high hardness and sufficient fracture toughness to avoid brittle fracture. Hard ceramic particles or fibers present in the matrix can withstand the cutting or abrasive action of the abrasive particles [21]. 
Six (6) types of coating systems (Table 6-1) on metallic surfaces were evaluated for abrasion and corrosion resistance at WVU-CFC using ASTM Taber test methods. Two of the coating systems (vinyl 3-a-z and vinyl 5-e-z) applied on standard 4"x4" metallic plates were obtained from the US Army corps of Engineers, which later on were trimmed to 3"X2" sizes. Other four coating systems consisting of composite or ceramic components evaluated in this study include: i) ARC 855 Coating, ii) ARC S2, iii) UHMW-PE plate (Ultra high-molecular weight polyethylene), and iv) F Coating. These coatings were applied on 3" X 2"sand-blasted metal plates. ARC 855 and ARC S2 coatings were applied on the sand-blasted metal plates with dimensions 3"X 2" and also on top of Vinyl-3-a-z and Vinyl-5-e-z coated metal plates at WVUCFC lab.
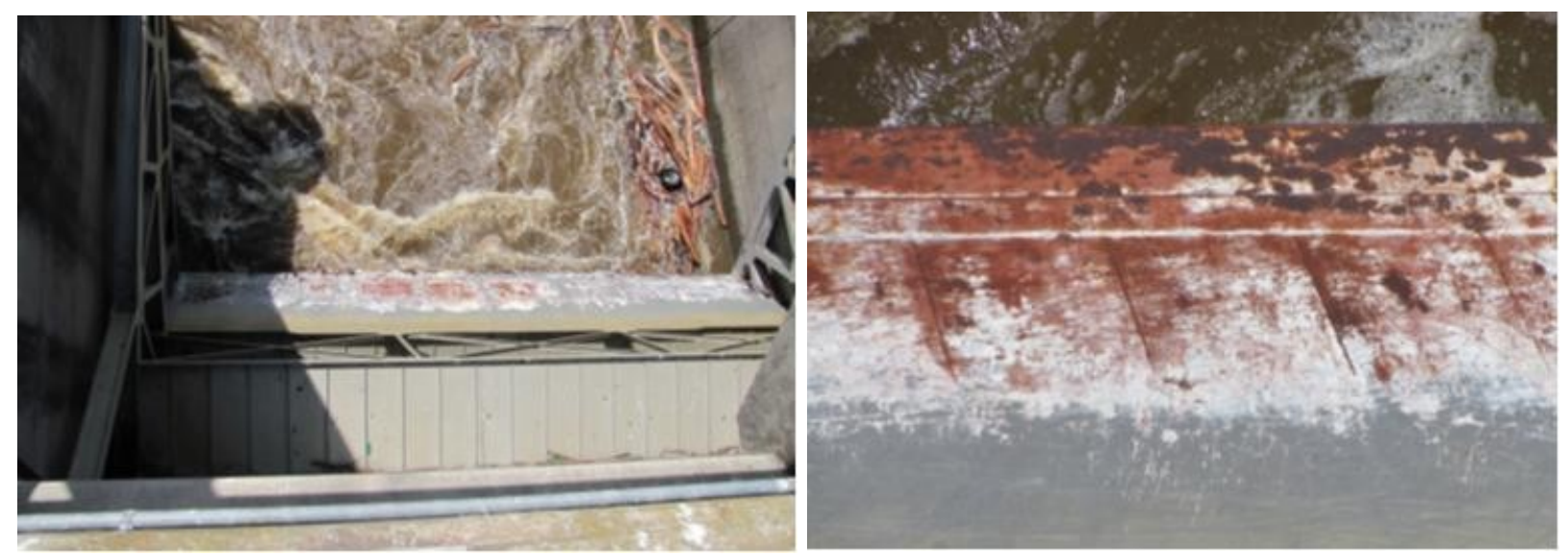

Figure 6.1: Debris that swirls around on the downstream side of the tainter gate (left); damage to the vinyl coating system caused by swirling debris (right) [5]

\subsection{Materials}

Six different types of abrasion resistant coating materials/systems were applied on sand blasted metallic plates in order to perform comparative abrasion tests on them. 
Table 6-1: Six types of coating systems

\begin{tabular}{|c|l|l|}
\hline No. & \multicolumn{1}{|c|}{ Coating System } & \multicolumn{1}{|c|}{ Source } \\
\hline 1 & Vinyl 3-a-z & USACE/Sherwin Williams \\
\hline 2 & Vinyl 5-e-z & USACE/Sherwin Williams \\
\hline 3 & ARC 855 Coating & A.W. Chesterton Company \\
\hline 4 & ARC S2 & A.W. Chesterton Company \\
\hline 5 & UHMW-PE (Ultra-High Molecular Weight Polyethylene) & $\begin{array}{l}\text { LinkTech Innovation/ } \\
\text { TSE-OKULEN }\end{array}$ \\
\hline 6 & Flexible (Fibrous) Polymer Coating & Rutgers University \\
\hline
\end{tabular}

\subsubsection{Vinyl 3-A-Z}

This coating system was obtained from U.S. Army Corps of Engineers (USACE). This coating system is specially formulated and designed for use on hydraulic structures primarily on locks and dams. This system is prevalently used in tainter gates in locks and dams. Vinyl 3-a-z coating system is achieved by coating the surface with vinyl based coatings for four times i.e. 1 coat of vinyl zinc rich primer, 1 coat of white vinyl, and 2 coats of aluminum vinyl.

\subsubsection{Vinyl 5-E-Z}

This coating system was also obtained from USACE. It is also specially designed for use on hydraulic structures primarily on locks and dams. Vinyl 5-e-z coating system is achieved by coating the surface with vinyl based coatings for four times i.e. 1 coat of vinyl zinc rich primer, 1 coat of gray vinyl, 1 coat of white vinyl, and final coat of gray vinyl.

\subsubsection{ARC 855}

It is an advanced ceramic composite developed and researched by A.W. Chesterton Company (Figure 6.2). It is composed of modified epoxy resin with a uniform blend of ceramics 
which is applied on metal surfaces in order to protect them from any kinds of corrosion, chemical attack, erosion and abrasion. It is a low viscous composite which can easily be applied with the help of a brush or roller without heat curing. It is usually applied in two coatings to provide extended resistance to abrasion.

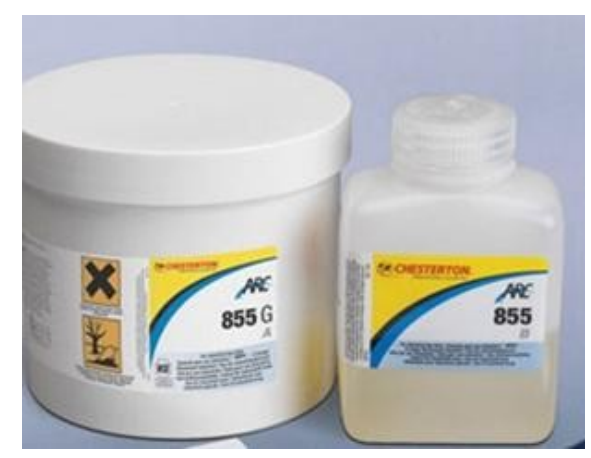

Figure 6.2: ARC ceramic composite coating

\subsubsection{ARC S2}

It is also an advanced ceramic composite developed and researched by A.W. Chesterton Company. It is a lower viscous composite than ARC 855 which is designed to be spray applied but may also be applied by a roller or brush. It is formulated for the resurfacing of metal components subjected to extreme corrosive or severe fluid flow conditions. It is a two coat system to provide extended wear life of the substrate. Once cured, ARC S2 provides a high gloss ceramic surface with good resistance to erosion and corrosion.

\subsubsection{UHMW-PE (Ultra high-molecular weight polyethylene)}

UHMW-PE bonded to a metallic plate specimens were obtained from LinkTech Innovation/ TSE-OKULEN Company. It is a high modulus polyethylene or high performance polyethylene having extremely long molecular chains with a molecular weight of 6 million g. 
/mol. It is a tough material with high impact strength. It is highly resistant to corrosive chemicals, has low moisture absorption and low coefficient of friction, is self-lubricating, and resistant to abrasion.

\subsubsection{Flexible (Fibrous) Polymer Coating}

It is a fibrous, flexible and flame-retardant polymer coating with a composition consisting of a cross-linked thermosetting polymer varnish, metal hydroxides, alcohol, and water. This coating is developed and patented by Rutgers University.

\subsection{Experimental details}

In order to study the abrasion resistance behavior of coatings, six different commercially available coating materials were applied on Zinc coated metallic plates of sizes 3" x 2". Photographs of metallic plates with different coatings for dry and wet conditionings are shown in Figure 6.5 and Figure 6.6, respectively. The thicknesses of each coating were measured in different locations using coating thickness gauge GL-7 PRO (Figure 6.4) which is an instrument developed by Prodig-tech company for measuring the thickness of a paint film or coating applied on either galvanized steel/aluminum or non-galvanized steel plate. It has a measuring range of 0 $\mu \mathrm{m}-2000 \mu \mathrm{m}$ and measuring resolution of $1 \mu \mathrm{m}-10 \mu \mathrm{m}$. It comes with a calibration plate and is used to calibrate the device i.e. zeroing the reading of an instrument. After calibration, the probe of an instrument is placed gently over the coated surface and the display shows the thickness of the coating in " $\mu \mathrm{m}$ ". The average of the thickness readings scanned in various locations is used as the coating thickness (Table 6-2). 


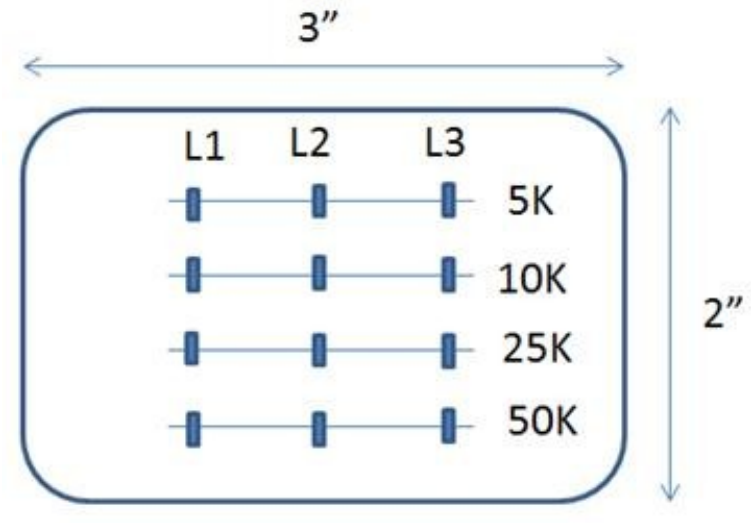

Figure 6.3: Dimension of the metal plate and locations (L1, L2, L3) of Scanning
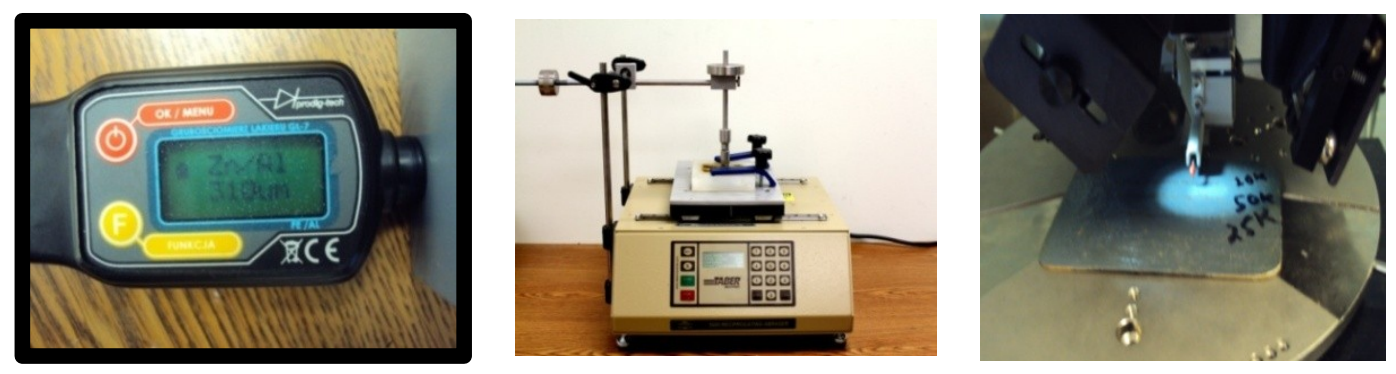

Figure 6.4: GL-7 PRO, TABER reciprocating abrader, profilometer (from left to right)

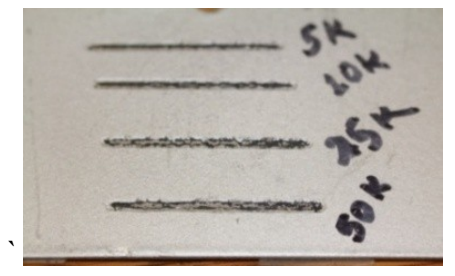

Vinyl 3-a-z

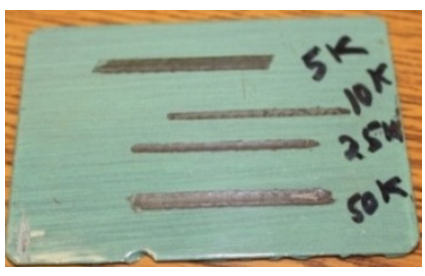

ARC S2

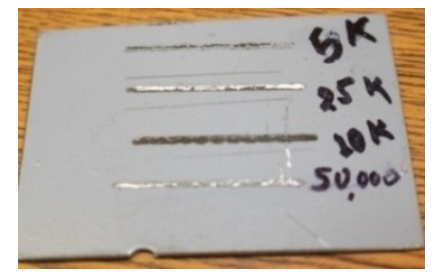

Vinyl 5-e-z

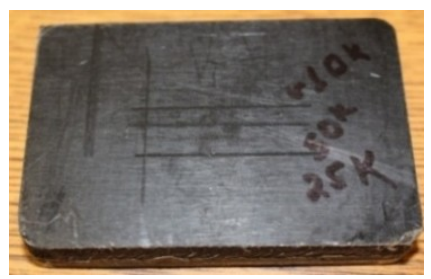

UHMW-PE

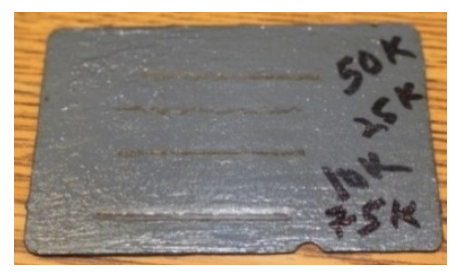

ARC 855

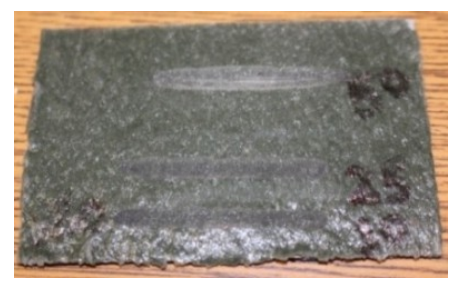

Flexible Polymer

Figure 6.5: Abraded coating specimens under no conditionings (i.e. dry surface) 
Table 6-2: Thicknesses of different coatings on the metal plates

\begin{tabular}{|l|l|c|}
\hline No. & Coating System & Thickness, mm (mils) \\
\hline 1 & Vinyl 3-a-z & $0.23(9.05)$ \\
\hline 2 & Vinyl 5-e-z & $0.25(9.84)$ \\
\hline 3 & ARC 855 & $0.30(11.81)$ \\
\hline 4 & ARC S2 & $0.249(9.80)$ \\
\hline 5 & UHMW-PE (Ultra high-molecular weight polyethylene) & $8.128(320)$ \\
\hline 6 & Flexible Polymer & $0.88(34.72)$ \\
\hline
\end{tabular}

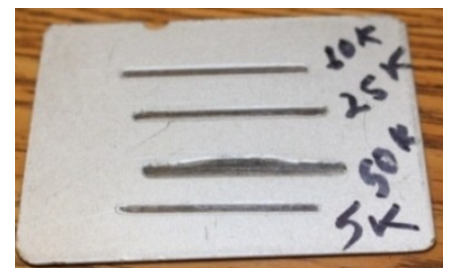

Vinyl 3-a-z

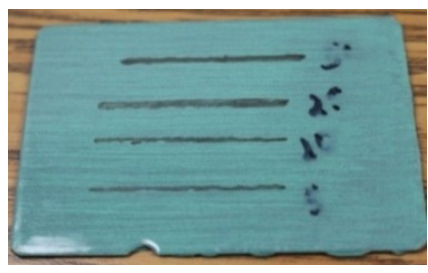

ARC S2

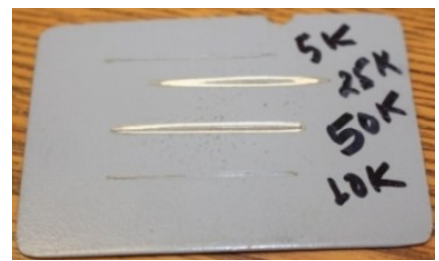

Vinyl 5-e-z

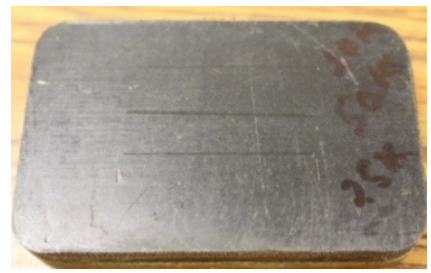

UHMW-PE

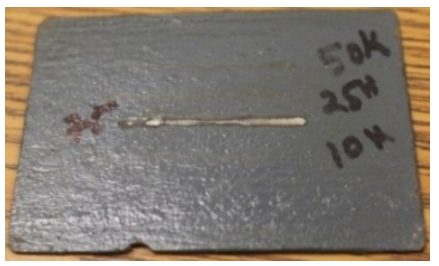

ARC 855

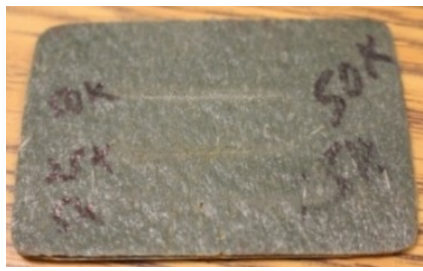

Flexible Polymer

Figure 6.6: Abraded coating specimens under water immersion conditions (i.e. wet surface)

The abrasion tests performed on the coated materials was two body abrasion with steel ball of diameter 3/8th of an inch $(9.652 \mathrm{~mm})$ being a counter body and the coated surface of different materials being a base body. The instrument used for two body abrasion was TABER reciprocating abrader with modified wearaser in the form of steel balls. Different coatings applied on the metallic plates were subjected to abrasion under no conditionings and under wet conditionings (i.e. under normal water at room temperature) for different cycles of abrasion (i.e. $5000,10000,25000,50000$ cycles) in TABER reciprocating abrader at 45 cycles per minute and 
for a stroke length of $36 \mathrm{~mm}$ with a constant normal load of $5 \mathrm{~N}$ on all the specimens during abrasion. Steel balls were replaced in every 10,000 cycles before they themselves got wear down in order to maintain the minimum point contact between the steel ball and the coating surface.

After abrading each of the coating systems for 5000, 10000, 25000, and 50000 cycles, the abraded surface profiles (the scar made by abrasion) of the coated metal plates were observed under the "Veeco Dektak 150" surface Profilometer, where stylus of the profilometer was made to run across the width of the scar at three different locations along the length of the scar measuring surface topography, roughness and step heights using contact profilometry techniques (Figure 6.7). This profilometer uses $640 \mathrm{X} 480$ pixel camera with $100 \mathrm{X}$ to $644 \mathrm{X}$ magnification for sample viewing and can measure two dimensional surfaces up to a depth of 21 mils. The lateral scan length can be varied from $55 \mathrm{~mm}$ to $200 \mathrm{~mm}$. The depth of abrasion on different coatings after certain number of cycles of abrasion measured from the profilometer is shown in Table 3.
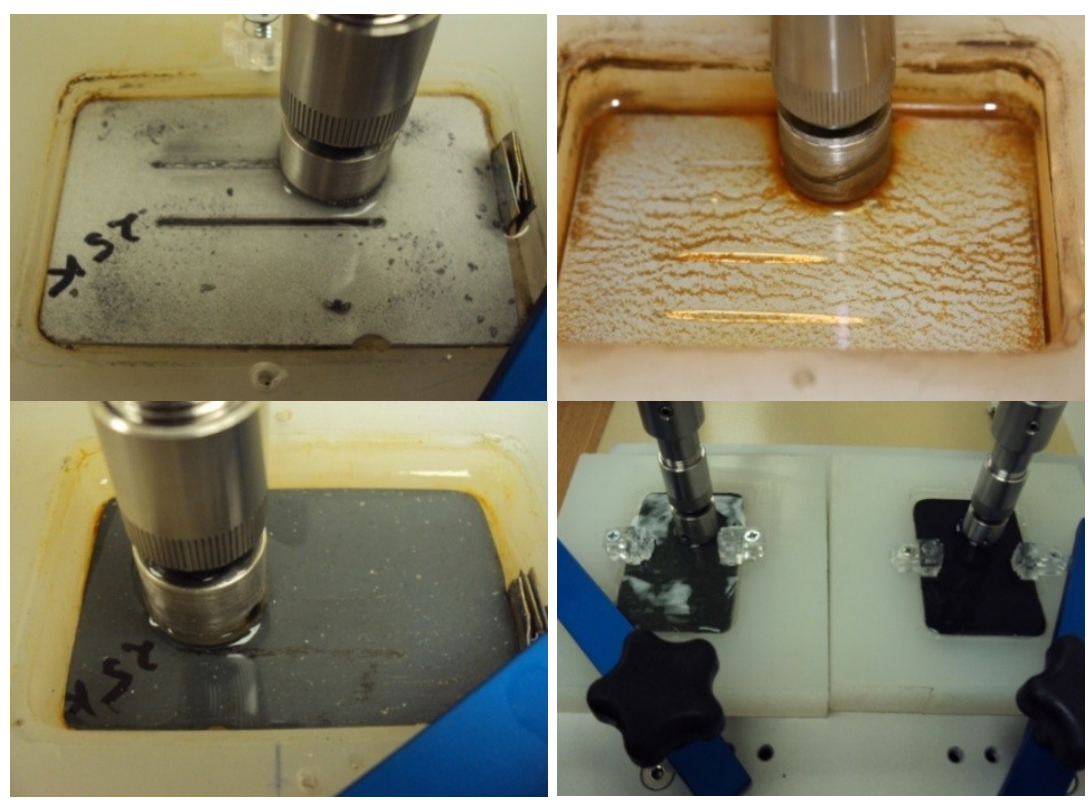

Figure 6.7: Abrasion on coating surface of vinyl 3-a-z, vinyl 5-e-z, ARC 855, flexible polymer, UHMW-PE (from top left to bottom right) 
In addition to the reciprocating Taber abrasion tests, the contact angles of the distilled water droplet on different coating surfaces were measured. The contact angle of liquid (distilled water) droplets was measured with a goniometer, which allows measuring the contact angle on the sessile drop of liquid. The droplet was deposited by a syringe pointed vertically down onto the sample surface, and a high resolution camera was used to capture the image, which can then be analyzed using image analysis software. When measuring contact angle, the amount of drops placed on the surface does not change the contact angle, anywhere from 1-10 drops can be placed and the measurement would be nearly same. However, with the single drop being placed at five different places over the coated surface and averaging out the angles of contact provides better accuracy which was used in this study.

\subsection{Theoretical background}

In the simplest form, the theory of abrasion [18] states that wear volume per unit sliding distance, $(\mathrm{Q})$ is related to the applied normal force, the distance traversed by the abrading body, and the resistance to plastic deformation (hardness) of the base body and is given by (Eqn 6.1).

$$
Q=\frac{V}{S}=\frac{K N}{H}
$$

Where, $\mathrm{V}$ is the volume abraded in $\mathrm{mm}^{3}, \mathrm{~S}$ is the sliding distance in meters, $\mathrm{K}$ is the dimensionless wear coefficient, $\mathrm{N}$ is the force applied normal to the contact in Newton and $\mathrm{H}$ is the hardness of the wearing material in $\mathrm{N} / \mathrm{mm}^{2}$ [34]. 
If wear coefficient $(\mathrm{k})$ takes into account the abrasive particles and material response, the typical way of computing wear index with a ball on disk test for a monolithic material is presented as Eqn 6.2 [35]:

$$
S * N=\frac{V}{K}
$$

Where $K$ is the wear rate with units of $\mathrm{mm} 3 /(\mathrm{m} \mathrm{N})$ and other parameters as defined under equation 1. While doing wear tests, if breakthrough of the coating is caused, it results in an abrasion of coating as well as of substrate. Thus, wear rate calculated is the combination of the coating and the substrate. In this case, wear rate is given by Eqn 6.3.

$$
S * N=\frac{V_{c}}{K_{c}}+\frac{V_{S}}{K_{S}}
$$

Where, $V_{c}$ and $V_{s}$ are volume abraded on coating and substrate respectively while $K_{c}$ and $K_{S}$ denote the wear rate of the coating and the substrate respectively.

According to the study [36], when the coated surface is abraded, the rate of surface wearing down $(d x / d t)$ as shown in Fig. 6 mainly depends upon tribological conditions to which the surface is subjected and the response of the coating material to these conditions. Ideally, the rate of wear can be written as Eqn 6.4.

$$
\frac{d x}{d t}=\psi \boldsymbol{r} k(\boldsymbol{r})
$$




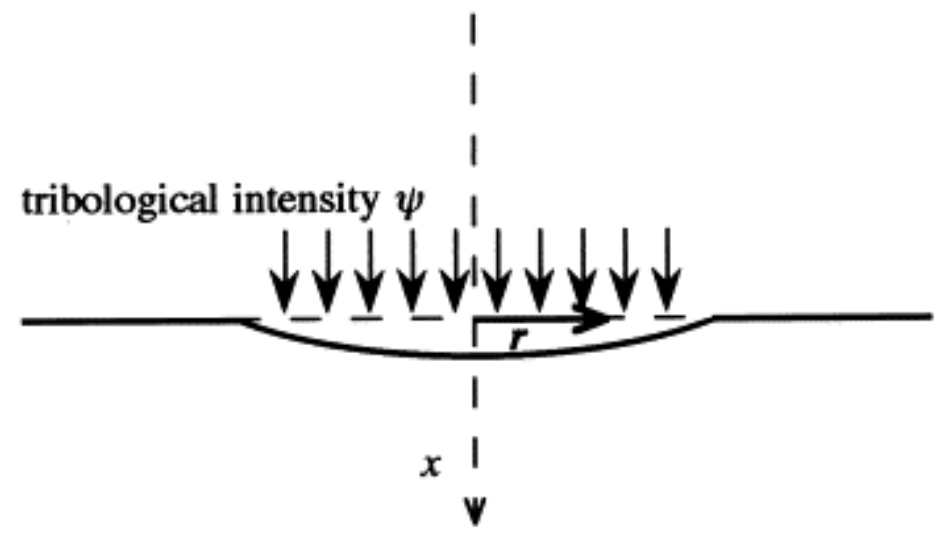

Figure 6.8: Wear down of the specimen surface during abrasion with tribological intensity $\psi[36]$

Where, $\psi r$ describes the tribological conditions or the severity of the mechanical stimulus applied on the material causing wear at any point on the surface defined by position vector $\boldsymbol{r}$ and $k(\boldsymbol{r})$ describes the response of the material at that point. The quantity $k$ is taken as specific wear rate and is expressed in sliding abrasion as "volume loss per unit sliding distance, per unit normal load" with units of $\mathrm{mm}^{3} /(m \mathrm{~N})$. Unless there are changes in wear mechanism, $k$ is often taken as constant for a certain experimental conditions. If the specimen or a part of it is subjected to abrasion under conditions of constant tribological intensity $(\psi)$, then the depth of the material $(x)$ removed after an abrasion test for duration $\mathrm{T}$ is shown in Eqn 6.5.

$$
x t=\psi{ }_{0}^{T} k d t=\psi k T
$$

In order to find out the tribological intensity in case of abrasive wear, the model given by [37] is used which states that the volume lost from the surface per unit sliding distance $(\mathrm{Q})$ is linearly proportional to the normal load, $\mathrm{N}$ (Eqn 6.6).

$$
Q=k N
$$


Where, $k$ is the specific wear rate and $N$ is the applied normal load. Rate of linear recession of the surface $(d x / d t)$ (Eqn 6.7) can be obtained by re-arranging Equations 6.4 and 6.6.

$$
\frac{d x}{d t}=k \frac{U N}{A}
$$

Where, $A$ is the area of contact between the sliding surfaces and $U$ is the relative sliding velocity. By comparing the equations, the tribological intensity can be found as (Eqn 6.8):

$$
\psi=\frac{U N}{A}
$$

This equation can be used to find out tribological intensity in product of sliding speed $(U)$ and contact pressure $(N / A)$. In reciprocating Taber abrader, both $\psi$ and worn area are nominally constant during the whole test [36].

\subsection{Results \& discussions}

Coating thickness was different for different specimens and TABER reciprocating abrasion tests were performed such that complete breakthrough of the coating was not made. The scar depths after the abrasion test shown in following Table 6-3 to Table 6-8 are less than the initial coating thickness shown in Table 6-2. As the study is focused mainly on the abrasion resistant characteristics of the coatings, but not of the substrate, complete breakthrough was not made. The plots for scar depth vs. lateral scan length for different coatings after 50,000 cycles of abrasion are shown in Figure 6.9 to Figure 6.14). Further, the abraded volume of the coating after $(5 \mathrm{k}, 10 \mathrm{k}, 25 \mathrm{k}$, and $50 \mathrm{k})$ number of abrasion cycles is shown in Table 6-9. 
Table 6-3: Results (vinyl 3-a-z coating)

\begin{tabular}{|c|c|c|c|c|c|c|c|}
\hline \multirow[t]{2}{*}{ No } & \multirow[t]{2}{*}{ Material } & \multirow[t]{2}{*}{ Surface } & \multirow{2}{*}{$\begin{array}{l}\text { No. of } \\
\text { Cycles }\end{array}$} & \multirow[t]{2}{*}{ Location } & \multicolumn{3}{|c|}{ Depth of Abrasion } \\
\hline & & & & & $\mathbf{A}^{0}$ & Avg. $\left(A^{0}\right)$ & Avg.(mils) \\
\hline \multirow{24}{*}{1.} & \multirow{24}{*}{$\begin{array}{l}\text { Vinyl 3-a-z } \\
\text { (9.45 mils) }\end{array}$} & \multirow{12}{*}{$\begin{array}{c}\text { Dry } \\
\text { Surface }\end{array}$} & \multirow{3}{*}{$5 \mathrm{k}$} & L1 & 850744 & \multirow{3}{*}{828282} & \multirow{3}{*}{3.26} \\
\hline & & & & $\mathrm{L} 2$ & 766990 & & \\
\hline & & & & L3 & 867111 & & \\
\hline & & & \multirow{3}{*}{$10 \mathrm{k}$} & L1 & 1147531 & \multirow{3}{*}{1041371} & \multirow{3}{*}{4.09} \\
\hline & & & & L2 & 1048931 & & \\
\hline & & & & L3 & 927652 & & \\
\hline & & & \multirow{3}{*}{$25 \mathrm{k}$} & $\mathrm{L} 1$ & 1017452 & \multirow{3}{*}{1107284} & \multirow{3}{*}{4.36} \\
\hline & & & & L2 & 1100621 & & \\
\hline & & & & L3 & 1203780 & & \\
\hline & & & \multirow{3}{*}{$50 \mathrm{k}$} & L1 & 1226653 & \multirow{3}{*}{1223205} & \multirow{3}{*}{4.82} \\
\hline & & & & L2 & 1260105 & & \\
\hline & & & & L3 & 1182858 & & \\
\hline & & \multirow{12}{*}{$\begin{array}{c}\text { Wet } \\
\text { Surface }\end{array}$} & \multirow{3}{*}{$5 \mathrm{k}$} & L1 & 1113780 & \multirow{3}{*}{114180} & \multirow{3}{*}{4.49} \\
\hline & & & & $\mathrm{L} 2$ & 1152225 & & \\
\hline & & & & L3 & 1157232 & & \\
\hline & & & \multirow{3}{*}{$10 \mathrm{k}$} & L1 & 1269710 & \multirow{3}{*}{1295590} & \multirow{3}{*}{5.10} \\
\hline & & & & L2 & 1325026 & & \\
\hline & & & & L3 & 1292034 & & \\
\hline & & & \multirow{3}{*}{$25 \mathrm{k}$} & L1 & 1429581 & \multirow{3}{*}{1456905} & \multirow{3}{*}{5.74} \\
\hline & & & & L2 & 1495564 & & \\
\hline & & & & L3 & 1445569 & & \\
\hline & & & \multirow{3}{*}{$50 \mathrm{k}$} & L1 & 1592221 & & \\
\hline & & & & L2 & 1559366 & 1544469 & 6.08 \\
\hline & & & & L3 & 1481817 & & \\
\hline
\end{tabular}

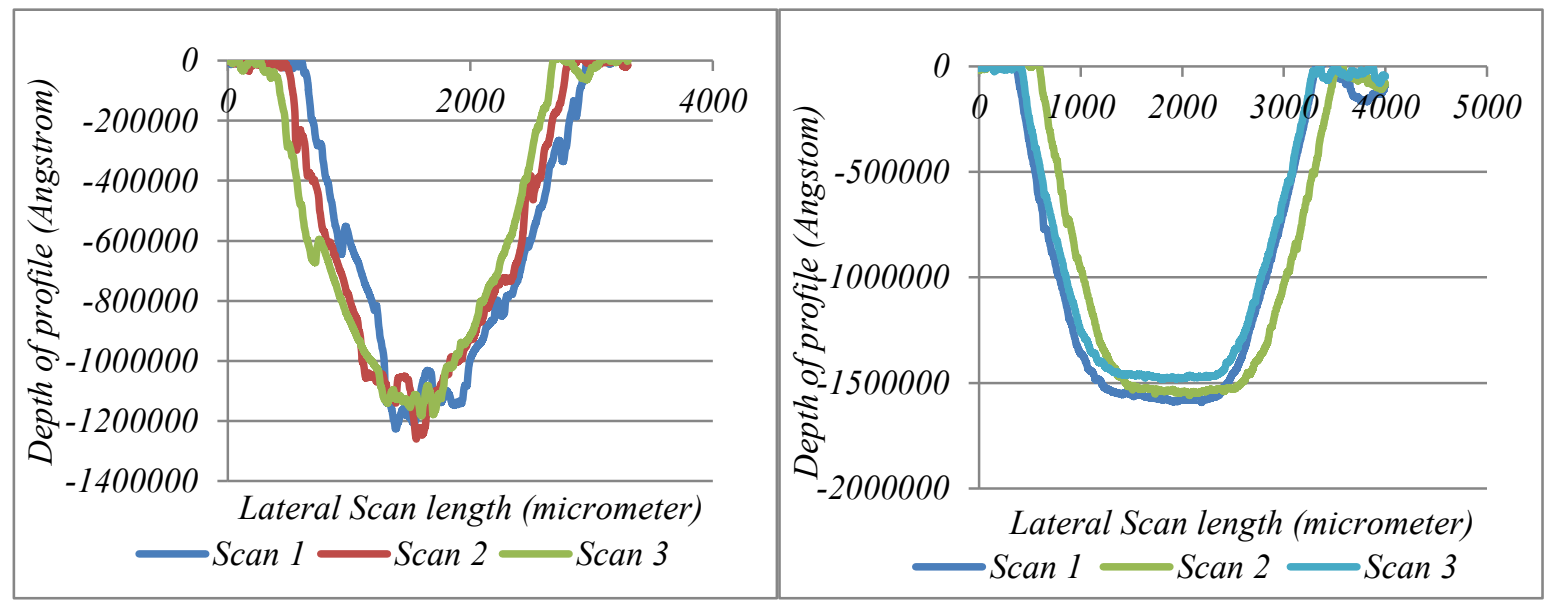

Figure 6.9: Depth profile of vinyl 3-a-z coating after 50,000 cycles of abrasion (dry surface, wet surface) 
Table 6-4: Results (vinyl 5-e-z coating)

\begin{tabular}{|c|c|c|c|c|c|c|c|}
\hline \multirow[t]{2}{*}{ No } & \multirow[t]{2}{*}{ Material } & \multirow[t]{2}{*}{ Surface } & \multirow{2}{*}{$\begin{array}{l}\text { No. of } \\
\text { Cycles }\end{array}$} & \multirow[t]{2}{*}{ Location } & \multicolumn{3}{|c|}{ Depth of Abrasion } \\
\hline & & & & & $\mathbf{A}^{0}$ & $\operatorname{Avg} .\left(A^{0}\right)$ & Avg. (mils) \\
\hline \multirow{24}{*}{2.} & \multirow{24}{*}{$\begin{array}{l}\text { Vinyl 5-e-z } \\
\text { (9.84 mils) }\end{array}$} & \multirow{12}{*}{$\begin{array}{c}\text { Dry } \\
\text { Surface }\end{array}$} & \multirow{3}{*}{$5 \mathrm{k}$} & L1 & 178051 & \multirow{3}{*}{157258} & \multirow{3}{*}{0.62} \\
\hline & & & & L2 & 138521 & & \\
\hline & & & & L3 & 155201 & & \\
\hline & & & \multirow{3}{*}{$10 \mathrm{k}$} & L1 & 247983 & \multirow{3}{*}{178970} & \multirow{3}{*}{0.68} \\
\hline & & & & L2 & 96572 & & \\
\hline & & & & L3 & 192356 & & \\
\hline & & & \multirow{3}{*}{$25 \mathrm{k}$} & $\mathrm{L} 1$ & 424156 & \multirow{3}{*}{434262} & \multirow{3}{*}{1.71} \\
\hline & & & & $\mathrm{L} 2$ & 437423 & & \\
\hline & & & & L3 & 441206 & & \\
\hline & & & \multirow{3}{*}{$50 \mathrm{k}$} & $\overline{\mathrm{L} 1}$ & 558622 & \multirow{3}{*}{540683} & \multirow{3}{*}{2.13} \\
\hline & & & & L2 & 674655 & & \\
\hline & & & & L3 & 388774 & & \\
\hline & & \multirow{12}{*}{$\begin{array}{c}\text { Wet } \\
\text { Surface }\end{array}$} & \multirow{3}{*}{$5 \mathrm{k}$} & L1 & 181435 & \multirow{3}{*}{207214} & \multirow{3}{*}{0.82} \\
\hline & & & & L2 & 246411 & & \\
\hline & & & & L3 & 193795 & & \\
\hline & & & \multirow{3}{*}{$10 \mathrm{k}$} & $\mathrm{L} 1$ & 385639 & \multirow{3}{*}{431547} & \multirow{3}{*}{1.70} \\
\hline & & & & L2 & 477984 & & \\
\hline & & & & L3 & 431017 & & \\
\hline & & & \multirow{3}{*}{$25 \mathrm{k}$} & L1 & 908872 & \multirow{3}{*}{1097973} & \multirow{3}{*}{4.32} \\
\hline & & & & L2 & 1166895 & & \\
\hline & & & & L3 & 1218152 & & \\
\hline & & & \multirow{3}{*}{$50 \mathrm{k}$} & L1 & 1352780 & & \\
\hline & & & & L2 & 1657025 & 1552589 & 6.11 \\
\hline & & & & L3 & 1647962 & & \\
\hline
\end{tabular}
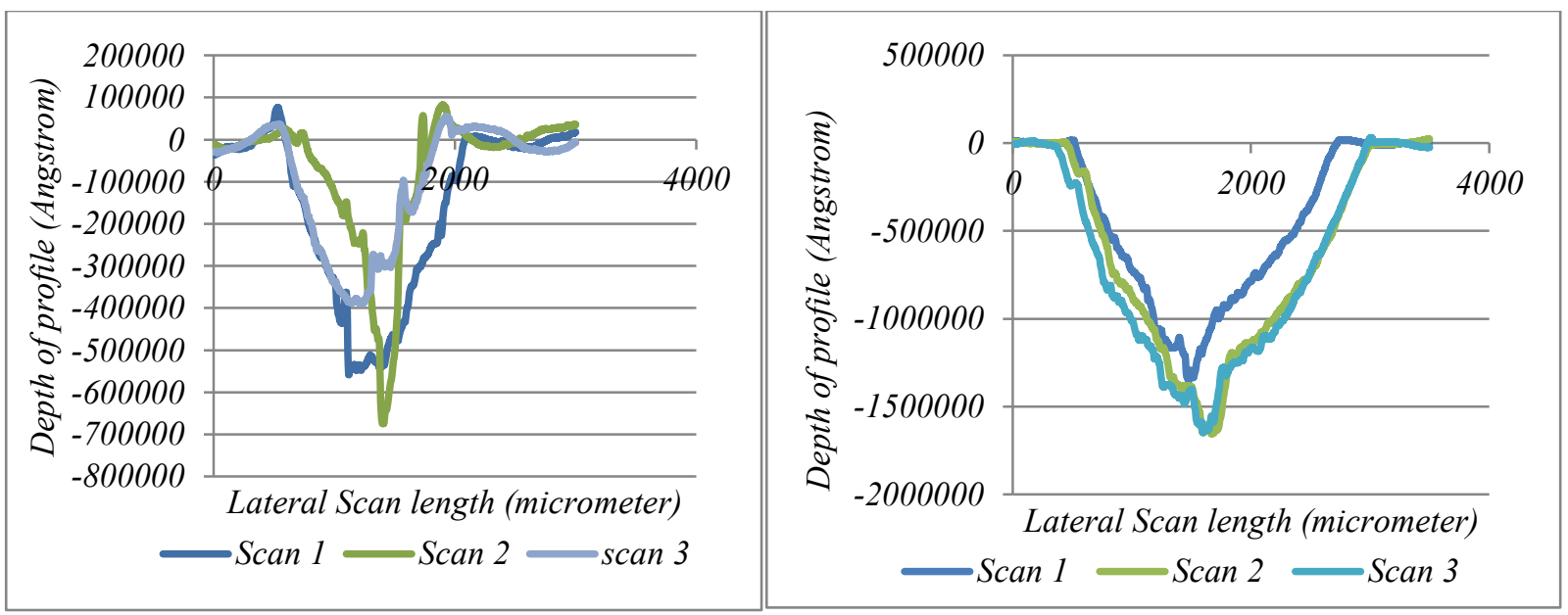

Figure 6.10: Depth profile of vinyl 5-e-z coating after 50,000 cycles of abrasion (dry surface, wet surface) 
Table 6-5: Results (ARC 855 coating)

\begin{tabular}{|c|c|c|c|c|c|c|c|}
\hline \multirow[t]{2}{*}{ No } & \multirow[t]{2}{*}{ Material } & \multirow[t]{2}{*}{ Surface } & \multirow{2}{*}{$\begin{array}{l}\text { No. of } \\
\text { Cycles }\end{array}$} & \multirow[t]{2}{*}{ Location } & \multicolumn{3}{|c|}{ Depth of Abrasion } \\
\hline & & & & & $\mathrm{A}^{0}$ & $\operatorname{Avg} .\left(A^{0}\right)$ & Avg. (mils) \\
\hline \multirow{18}{*}{3.} & \multirow{18}{*}{$\begin{array}{c}\text { ARC } 855 \\
\text { (11.8mils) }\end{array}$} & \multirow{9}{*}{$\begin{array}{c}\text { Dry } \\
\text { Surface }\end{array}$} & \multirow{3}{*}{$10 \mathrm{k}$} & $\mathrm{L} 1$ & 163651 & \multirow{3}{*}{196440} & \multirow{3}{*}{0.77} \\
\hline & & & & L2 & 229229 & & \\
\hline & & & & L3 & - & & \\
\hline & & & \multirow{3}{*}{$25 \mathrm{k}$} & $\overline{\mathrm{L} 1}$ & 218787 & \multirow{3}{*}{276231} & \multirow{3}{*}{1.09} \\
\hline & & & & L2 & 323328 & & \\
\hline & & & & L3 & 286578 & & \\
\hline & & & \multirow{3}{*}{$50 \mathrm{k}$} & $\mathrm{L} 1$ & 369762 & \multirow{3}{*}{394715} & \multirow{3}{*}{1.55} \\
\hline & & & & L2 & 420488 & & \\
\hline & & & & L3 & 393894 & & \\
\hline & & \multirow{9}{*}{$\begin{array}{c}\text { Wet } \\
\text { Surface }\end{array}$} & \multirow{3}{*}{$10 \mathrm{k}$} & $\mathrm{L} 1$ & 125557 & \multirow{3}{*}{116927} & \multirow{3}{*}{0.46} \\
\hline & & & & L2 & 133923 & & \\
\hline & & & & L3 & 91299 & & \\
\hline & & & \multirow{3}{*}{$25 \mathrm{k}$} & $\mathrm{L} 1$ & 264143 & \multirow{3}{*}{282810} & \multirow{3}{*}{1.11} \\
\hline & & & & L2 & 297139 & & \\
\hline & & & & L3 & 287148 & & \\
\hline & & & \multirow{3}{*}{$50 \mathrm{k}$} & L1 & 378463 & \multirow{3}{*}{330894} & \multirow{3}{*}{1.30} \\
\hline & & & & L2 & 356612 & & \\
\hline & & & & L3 & 257606 & & \\
\hline
\end{tabular}
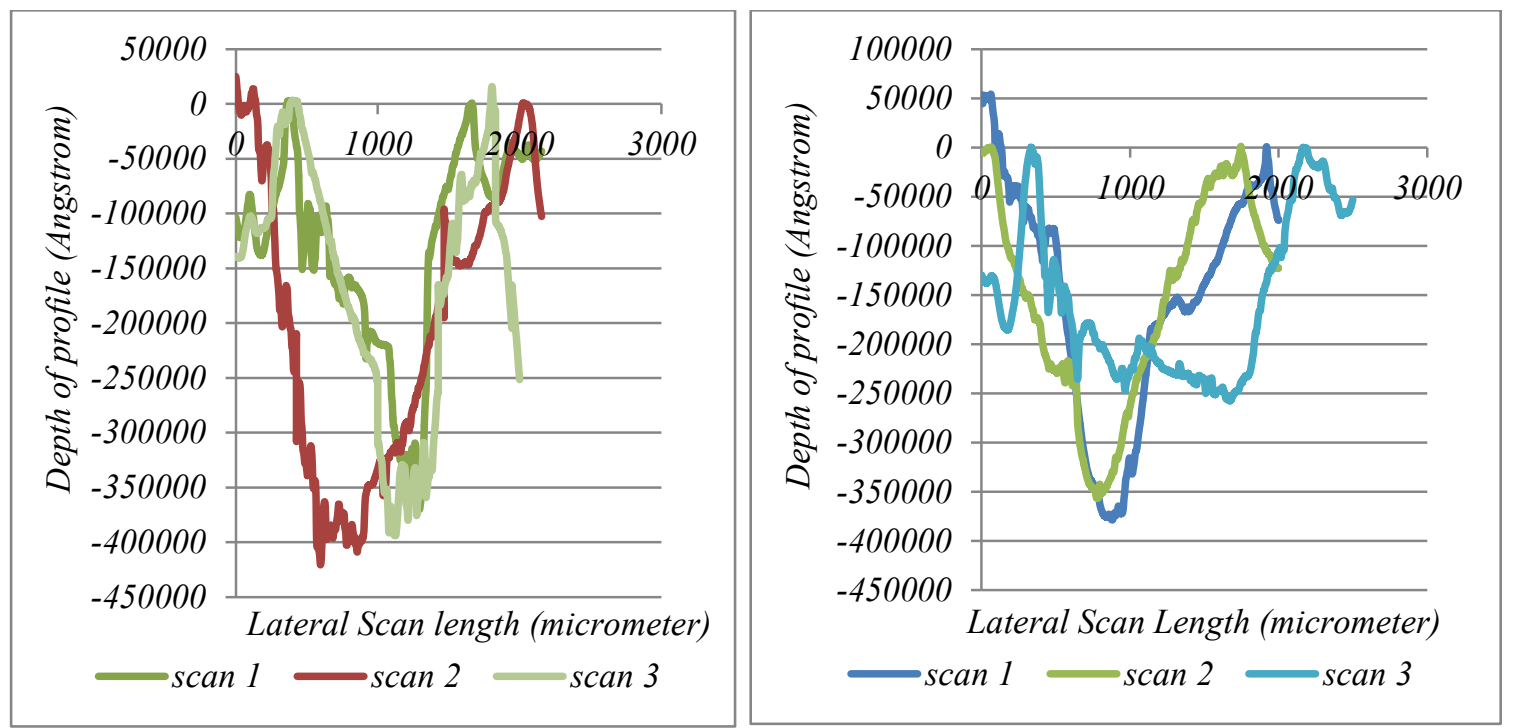

Figure 6.11: Depth profile of ARC 855 coating after 50,000 cycles of abrasion (dry, wet surface) 
Table 6-6: Results (ARC S2 coating)

\begin{tabular}{|c|c|c|c|c|c|c|c|}
\hline \multirow[t]{2}{*}{ No } & \multirow[t]{2}{*}{ Material } & \multirow[t]{2}{*}{ Surface } & \multirow{2}{*}{$\begin{array}{l}\text { No. of } \\
\text { Cycles }\end{array}$} & \multirow[t]{2}{*}{ Location } & \multicolumn{3}{|c|}{ Depth of Abrasion } \\
\hline & & & & & $\mathbf{A}^{0}$ & Avg. $\left(A^{0}\right)$ & Avg. (mils) \\
\hline \multirow{18}{*}{6.} & \multirow{18}{*}{$\begin{array}{c}\text { ARC S2 } \\
(9.8 \text { mils })\end{array}$} & \multirow{9}{*}{$\begin{array}{c}\text { Dry } \\
\text { Surface }\end{array}$} & \multirow{3}{*}{$10 \mathrm{k}$} & L1 & 494460 & \multirow{3}{*}{506763} & \multirow{3}{*}{1.99} \\
\hline & & & & L2 & 530164 & & \\
\hline & & & & L3 & 495665 & & \\
\hline & & & \multirow{3}{*}{$25 \mathrm{k}$} & L1 & 558982 & \multirow{3}{*}{605046} & \multirow{3}{*}{2.38} \\
\hline & & & & L2 & 632160 & & \\
\hline & & & & L3 & 623994 & & \\
\hline & & & \multirow{3}{*}{$50 \mathrm{k}$} & L1 & 782135 & \multirow{3}{*}{812203} & \multirow{3}{*}{3.20} \\
\hline & & & & $\mathrm{L} 2$ & 857124 & & \\
\hline & & & & L3 & 797350 & & \\
\hline & & \multirow{9}{*}{$\begin{array}{c}\text { Wet } \\
\text { Surface }\end{array}$} & \multirow{3}{*}{$10 \mathrm{k}$} & $\overline{\mathrm{L} 1}$ & 14091 & \multirow{3}{*}{18132} & \multirow{3}{*}{0.07} \\
\hline & & & & L2 & 21580 & & \\
\hline & & & & L3 & 18725 & & \\
\hline & & & \multirow{3}{*}{$25 \mathrm{k}$} & L1 & 26398 & \multirow{3}{*}{34557} & \multirow{3}{*}{0.14} \\
\hline & & & & L2 & 44028 & & \\
\hline & & & & L3 & 33244 & & \\
\hline & & & \multirow{3}{*}{$50 \mathrm{k}$} & L1 & 77248 & \multirow{3}{*}{80898} & \multirow{3}{*}{0.32} \\
\hline & & & & L2 & 83227 & & \\
\hline & & & & L3 & 82218 & & \\
\hline
\end{tabular}
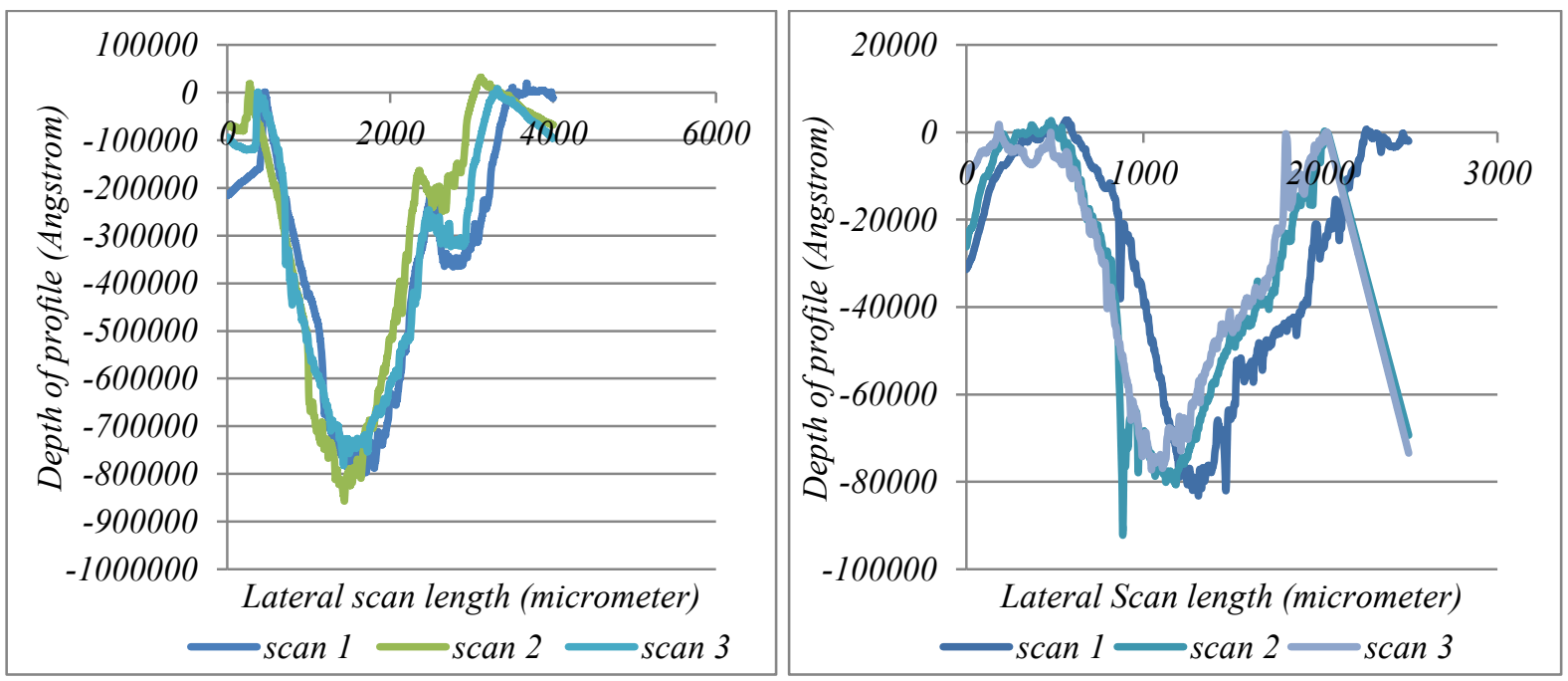

Figure 6.12: Depth profile of ARC S2 coating after 50,000 cycles of abrasion (dry, wet surface) 
Table 6-7: Results (UHMW-PE coating)

\begin{tabular}{|c|c|c|c|c|c|c|c|}
\hline \multirow[t]{2}{*}{ No } & \multirow[t]{2}{*}{ Material } & \multirow[t]{2}{*}{ Surface } & \multirow{2}{*}{$\begin{array}{l}\text { No. of } \\
\text { Cycles }\end{array}$} & \multirow[t]{2}{*}{ Location } & \multicolumn{3}{|c|}{ Depth of Abrasion } \\
\hline & & & & & $\mathrm{A}^{0}$ & Avg. $\left(A^{0}\right)$ & Avg. (mils) \\
\hline \multirow{18}{*}{6.} & \multirow{18}{*}{$\begin{array}{l}\text { UHMW- } \\
\text { PE } \\
\text { (320 mils) }\end{array}$} & \multirow{9}{*}{$\begin{array}{c}\text { Dry } \\
\text { Surface }\end{array}$} & \multirow{3}{*}{$10 \mathrm{k}$} & $\mathrm{L1}$ & 20856 & \multirow{3}{*}{20466} & \multirow{3}{*}{0.08} \\
\hline & & & & L2 & 20075.9 & & \\
\hline & & & & L3 & 21205 & & \\
\hline & & & \multirow{3}{*}{$25 \mathrm{k}$} & L1 & 36610 & \multirow{3}{*}{31905} & \multirow{3}{*}{0.13} \\
\hline & & & & $\mathrm{L} 2$ & 27925.9 & & \\
\hline & & & & L3 & 31178.7 & & \\
\hline & & & \multirow{3}{*}{$50 \mathrm{k}$} & $\mathrm{L1}$ & 42860.8 & \multirow{3}{*}{45839} & \multirow{3}{*}{0.18} \\
\hline & & & & $\mathrm{L} 2$ & 51524.7 & & \\
\hline & & & & L3 & 43132.2 & & \\
\hline & & \multirow{9}{*}{$\begin{array}{c}\text { Wet } \\
\text { Surface }\end{array}$} & \multirow{3}{*}{$10 \mathrm{k}$} & $\mathrm{L1}$ & 29434.5 & \multirow{3}{*}{27095} & \multirow{3}{*}{0.10} \\
\hline & & & & L2 & 24698.6 & & \\
\hline & & & & L3 & 27153.1 & & \\
\hline & & & \multirow{3}{*}{$25 \mathrm{k}$} & $\mathrm{L1}$ & 25988.9 & \multirow{3}{*}{28006} & \multirow{3}{*}{0.11} \\
\hline & & & & L2 & 29176.7 & & \\
\hline & & & & L3 & 28853 & & \\
\hline & & & \multirow{3}{*}{$50 \mathrm{k}$} & L1 & 43588.3 & \multirow{3}{*}{39988} & \multirow{3}{*}{0.16} \\
\hline & & & & L2 & 32972.7 & & \\
\hline & & & & L3 & 43402.7 & & \\
\hline
\end{tabular}
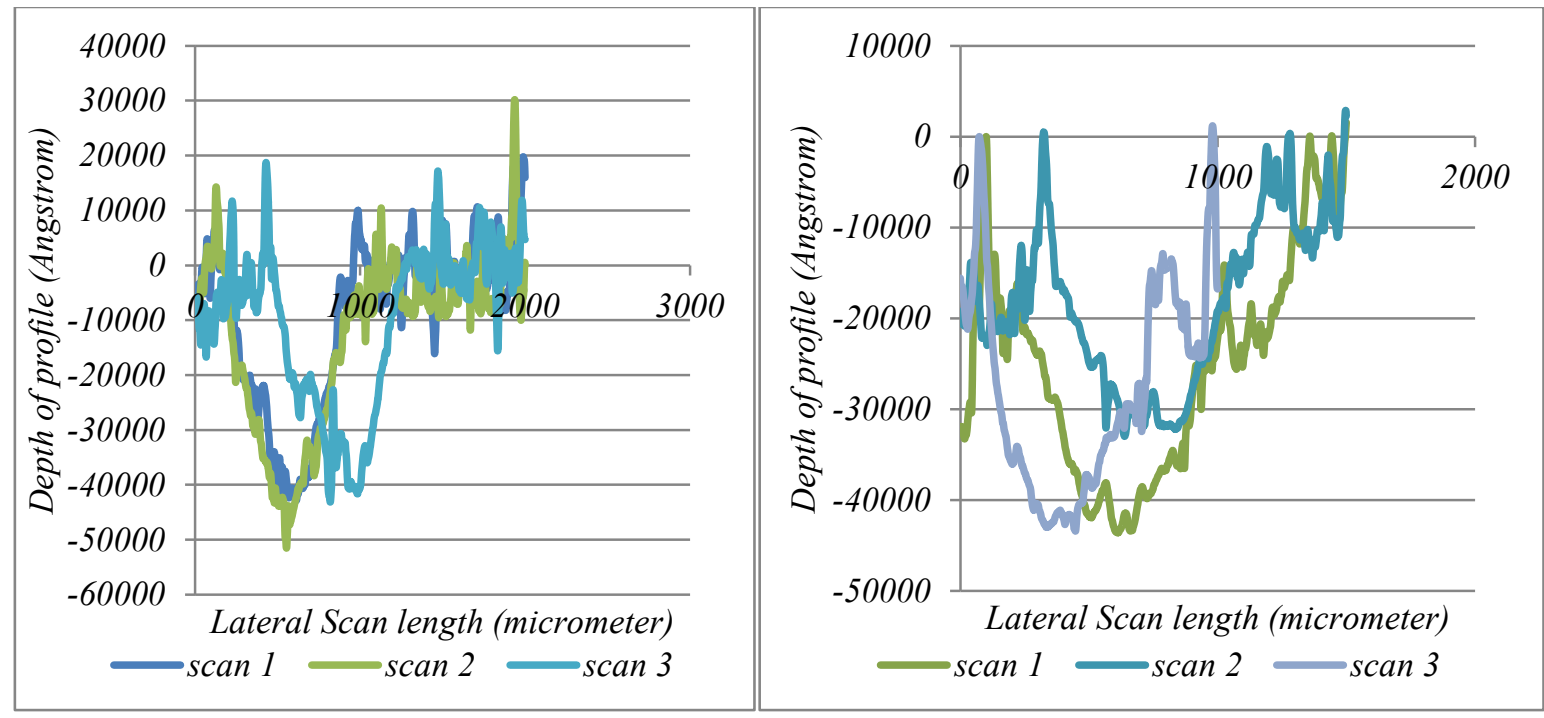

Figure 6.13: Depth profile of UHMW-PE coating after 50,000 cycles of abrasion (dry, wet surface) 
Table 6-8: Results (Rutgers coating)

\begin{tabular}{|c|c|c|c|c|c|c|c|}
\hline \multirow[t]{2}{*}{ No } & \multirow[t]{2}{*}{ Material } & \multirow[t]{2}{*}{ Surface } & \multirow{2}{*}{$\begin{array}{l}\text { No. of } \\
\text { Cycles }\end{array}$} & \multirow[t]{2}{*}{ Location } & \multicolumn{3}{|c|}{ Depth of Abrasion } \\
\hline & & & & & $\mathrm{A}^{0}$ & $\operatorname{Avg} .\left(A^{0}\right)$ & Avg. (mils) \\
\hline \multirow{17}{*}{7.} & \multirow{17}{*}{$\begin{array}{c}\text { Rutgers } \\
\text { Polymer } \\
(34.75 \\
\text { mils })\end{array}$} & \multirow{8}{*}{$\begin{array}{c}\text { Dry } \\
\text { surface }\end{array}$} & \multirow{3}{*}{$10 \mathrm{k}$} & L1 & 3208533 & \multirow{3}{*}{2470036} & \multirow{3}{*}{9.72} \\
\hline & & & & L2 & 2026045 & & \\
\hline & & & & L3 & 2175528 & & \\
\hline & & & \multirow{3}{*}{$25 \mathrm{k}$} & L1 & 4247109 & \multirow{3}{*}{3652352} & \multirow{3}{*}{14.38} \\
\hline & & & & L2 & 3304019 & & \\
\hline & & & & L3 & 3405928 & & \\
\hline & & & \multirow[b]{2}{*}{$50 \mathrm{k}$} & L1 & 4725406 & \multirow{2}{*}{$\begin{array}{c}> \\
4725406\end{array}$} & \multirow{2}{*}{$\begin{array}{c}> \\
18.60\end{array}$} \\
\hline & & & & $\mathrm{L} 2$ & Completely & & \\
\hline & & \multirow{9}{*}{$\begin{array}{c}\text { Wet } \\
\text { Surface }\end{array}$} & \multirow{3}{*}{$10 \mathrm{k}$} & L1 & 577316 & \multirow{3}{*}{808908} & \multirow{3}{*}{3.18} \\
\hline & & & & L2 & 1040500 & & \\
\hline & & & & L3 & - & & \\
\hline & & & \multirow{3}{*}{$25 \mathrm{k}$} & L1 & 999090 & \multirow{3}{*}{1164089} & \multirow{3}{*}{4.58} \\
\hline & & & & L2 & 1280078 & & \\
\hline & & & & L3 & 1213099 & & \\
\hline & & & \multirow{3}{*}{$50 \mathrm{k}$} & L1 & 2050265 & \multirow{3}{*}{2317210} & \multirow{3}{*}{9.12} \\
\hline & & & & L2 & 2298526 & & \\
\hline & & & & L3 & 2602839 & & \\
\hline
\end{tabular}

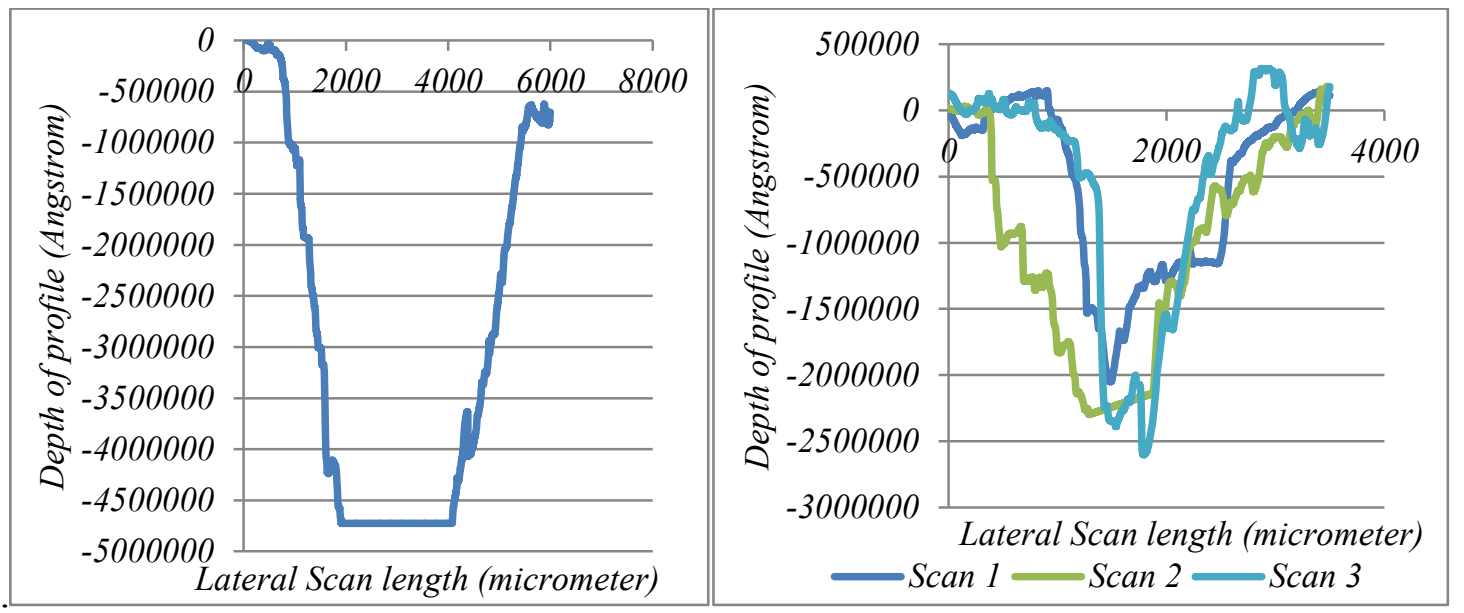

Figure 6.14: Depth profile of Rutgers coating after 50,000 cycles of abrasion (dry surface, wet surface)

Note: Rutgers coating surface under dry conditions was completely scratched at $50 \mathrm{~K}$ cycles and the coating depth was beyond the range of the profilometer 
Table 6-9: Abraded volume of the scar on both dry and wet surface of the coatings

\begin{tabular}{|c|c|c|c|c|c|c|c|}
\hline \multirow{3}{*}{ Coatings } & \multirow{3}{*}{$\begin{array}{l}\text { No. of } \\
\text { cycles }\end{array}$} & \multicolumn{2}{|c|}{$\begin{array}{c}\text { Avg. depth of scar } \\
\text { (mils) }\end{array}$} & \multicolumn{2}{|c|}{$\begin{array}{l}\text { Avg. c/s area of scar } \\
\qquad\left(\mathrm{mm}^{2}\right)\end{array}$} & \multicolumn{2}{|c|}{$\begin{array}{c}\text { Abraded volume of } \\
\text { coating }\left(\mathrm{mm}^{3}\right)\end{array}$} \\
\hline & & \multicolumn{2}{|c|}{ Conditioning } & \multicolumn{2}{|c|}{ Conditioning } & \multicolumn{2}{|c|}{ Conditioning } \\
\hline & & Dry & Wet & Dry & Wet & Dry & Wet \\
\hline \multirow{4}{*}{ Vinyl 3-a-z } & $5 \mathrm{k}$ & 3.261 & 4.492 & 0.1166 & 0.2022 & 4.197 & 7.279 \\
\hline & $10 \mathrm{k}$ & 4.099 & 5.101 & 0.1344 & 0.2575 & 4.839 & 9.271 \\
\hline & $25 \mathrm{k}$ & 4.359 & 5.736 & 0.1484 & 0.2968 & 5.342 & 10.68 \\
\hline & $50 \mathrm{k}$ & 4.816 & 6.081 & 0.1729 & 0.3356 & 6.225 & 12.08 \\
\hline \multirow{4}{*}{ Vinyl 5-e-z } & $5 \mathrm{k}$ & 0.619 & 0.816 & 0.0063 & 0.0135 & 0.2286 & 0.4857 \\
\hline & $10 \mathrm{k}$ & 0.705 & 1.699 & 0.0259 & 0.0407 & 0.8606 & 1.465 \\
\hline & $25 \mathrm{k}$ & 1.710 & 4.323 & 0.0428 & 0.1539 & 1.542 & 5.539 \\
\hline & $50 \mathrm{k}$ & 2.129 & 6.112 & 0.0466 & 0.2122 & 1.688 & 7.641 \\
\hline \multirow{3}{*}{ ARC 855} & $10 \mathrm{k}$ & 0.773 & 0.460 & 0.0109 & 0.0038 & 0.3913 & 0.1351 \\
\hline & $25 \mathrm{k}$ & 1.087 & 1.113 & 0.0255 & 0.0157 & 0.9183 & 0.5642 \\
\hline & $50 \mathrm{k}$ & 1.554 & 1.303 & 0.0326 & 0.0299 & 1.174 & 1.075 \\
\hline \multirow{3}{*}{ ARC S2 } & $10 \mathrm{k}$ & 1.995 & 0.071 & 0.0497 & 0.0005 & 1.790 & 0.0190 \\
\hline & $25 \mathrm{k}$ & 2.382 & 0.136 & 0.0689 & 0.0019 & 2.481 & 0.0671 \\
\hline & $50 \mathrm{k}$ & 3.198 & 0.318 & 0.1181 & 0.0064 & 4.250 & 0.2317 \\
\hline \multirow{3}{*}{$\begin{array}{l}\text { UHMW- } \\
\text { PE }\end{array}$} & $10 \mathrm{k}$ & 0.081 & 0.107 & 0.0007 & 0.0014 & 0.0236 & 0.0498 \\
\hline & $25 \mathrm{k}$ & 0.126 & 0.110 & 0.0013 & 0.0018 & 0.0455 & 0.0634 \\
\hline & $50 \mathrm{k}$ & 0.180 & 0.157 & 0.0021 & 0.0026 & 0.0755 & 0.0948 \\
\hline \multirow{3}{*}{$\begin{array}{l}\text { Flexible } \\
\text { polymer }\end{array}$} & $10 \mathrm{k}$ & 9.725 & 3.185 & 0.3968 & 0.0593 & 14.29 & 2.134 \\
\hline & $25 \mathrm{k}$ & 14.38 & 4.583 & 0.7803 & 0.0956 & 28.09 & 3.443 \\
\hline & $50 \mathrm{k}$ & $>18.60$ & 9.123 & 1.7370 & 0.3055 & 62.55 & 10.99 \\
\hline
\end{tabular}

Vinyl 3-a-z coated specimens did not perform well under both dry surface (i.e. at room temperature \& no water) and wet surface abrasion (i.e. underwater at room temperature) (Figure 6.15). This is the coating which is being primarily used in hydraulic structures and tainter gates in lock \& dams to prevent abrasion. The second coating primarily being used in such structures performed well under dry surface abrasion, with volume lost being just $1.676 \mathrm{~mm}^{3}$ after $50 \mathrm{k}$ 
cycles of abrasion but abrasion of these samples underwater performed poorly with $7.641 \mathrm{~mm}^{3}$ of material being lost after 50k cycles of abrasion. The volume lost was seen to increase with higher abrasion cycles with visible increase in coating wear down.

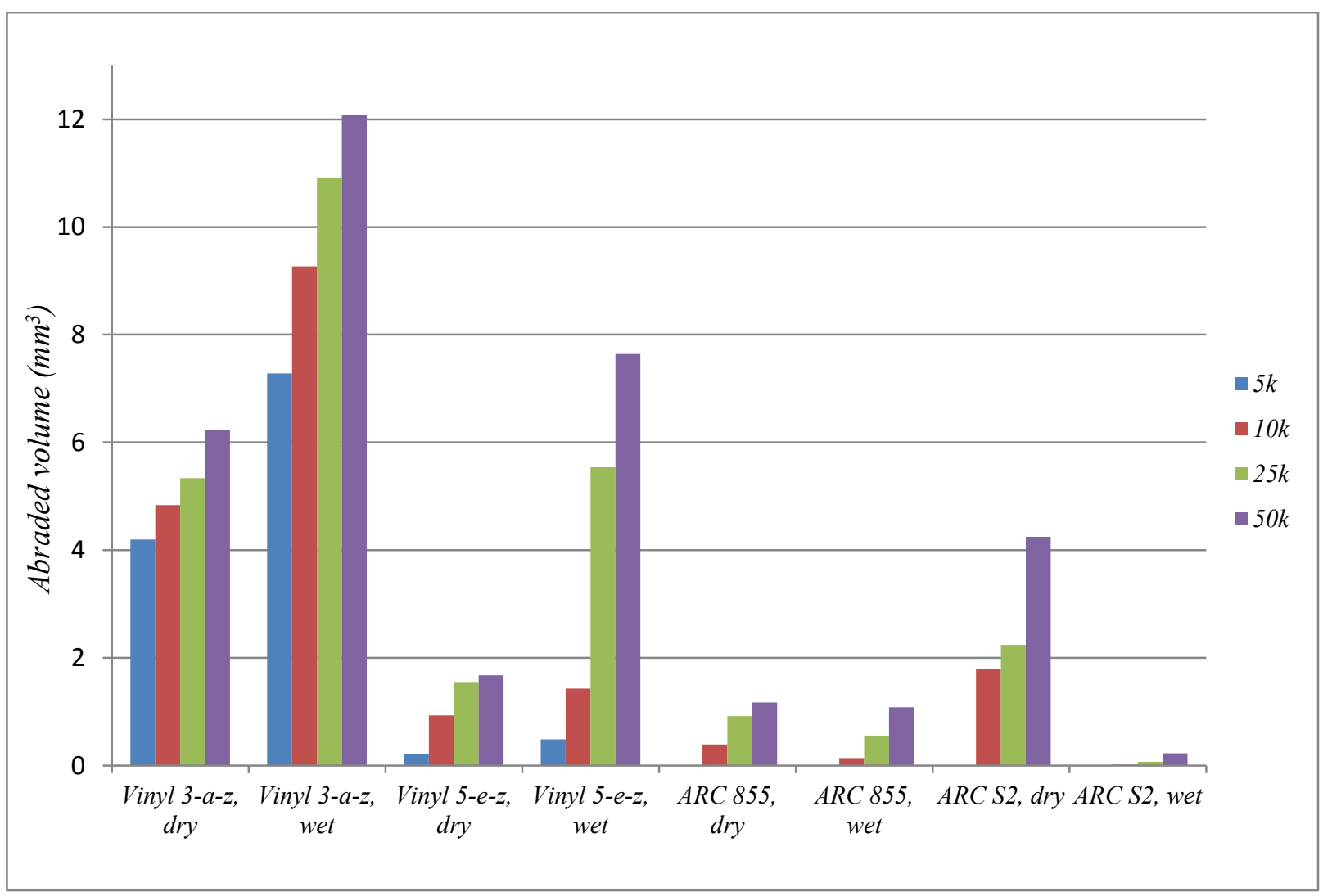

Figure 6.15: Abraded volume of coatings after different cycles of abrasion under dry/wet conditionings

ARC 855 and ARC S2 coated specimens performed very well, with both being better under wet surface abrasion (Figure 6.15 and Table 6-9). UHMW-PE material bonded specimens were excellent in withstanding abrasive forces. The material wear after $50 \mathrm{k}$ cycles of abrasion under dry and wet surface abrasion was observed to be just $0.0755 \mathrm{~mm}^{3}$ and 0.0948 $\mathrm{mm}^{3}$ respectively (Figure 6.16). 

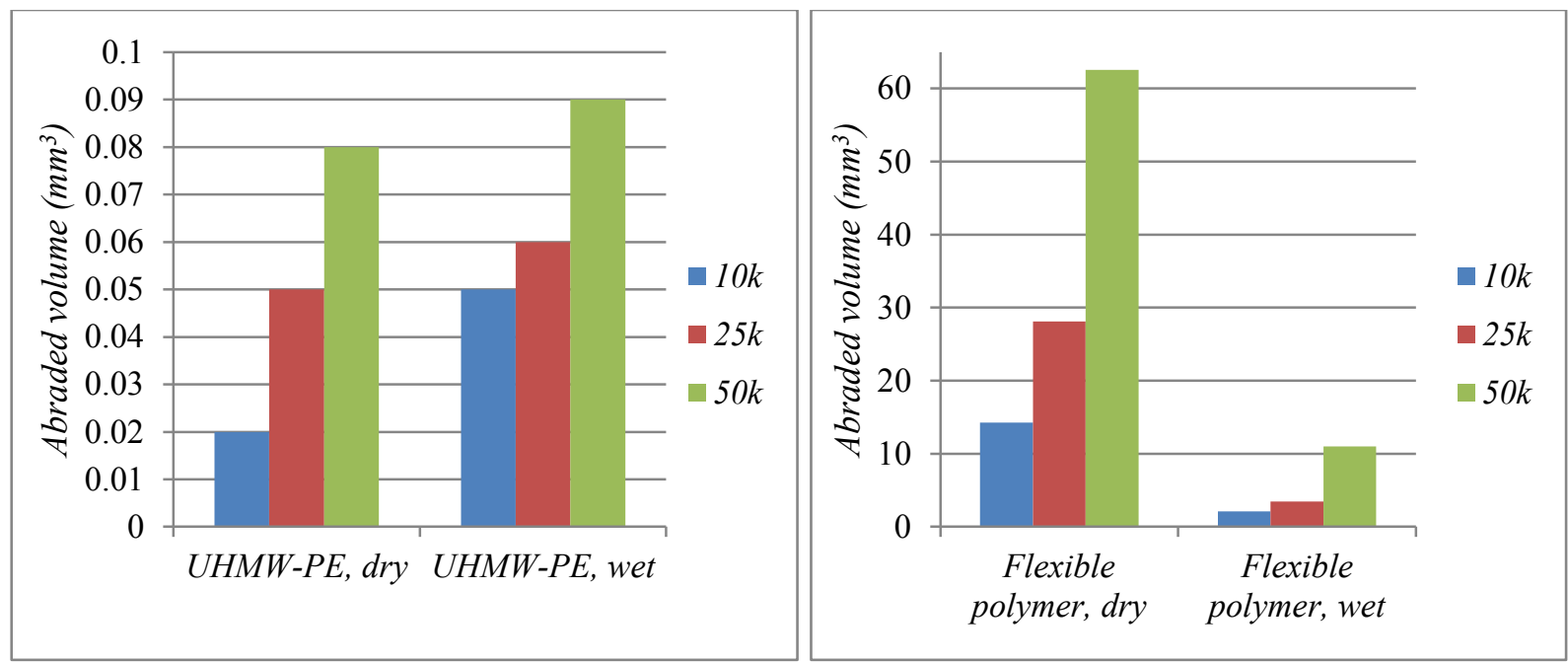

Figure 6.16: Abraded volume of coatings after different cycles of abrasion under dry/wet conditions

Specimens with flexible polymer coat wear down completely after 50k cycles of dry surface abrasion but performed better while under wet surface abrasion (Figure 6.16).

\subsection{Wear rate for different coatings}

Initial wear rate for Vinyl 3-a-z coated specimens is high in both dry and wet conditionings. During the test, large amount of wear debris was observed clearly just after few initial cycles of abrasion. For Vinyl 3-a-z specimens abraded at dry conditions, the wear is high at initial 10k cycles of abrasion but after that increase in wear is very less and constant. For Vinyl 3-a-z coated specimens abraded underwater, the wear is twice that of the dry condition with almost similar wear pattern (Figure 6.17 and Table 6-10). According to Taylor's law, initial wear rate is relatively high during break-in, followed by a long period of uniform wear at low rate and finally a brief period of increased wear rate just before complete break through [16]. 


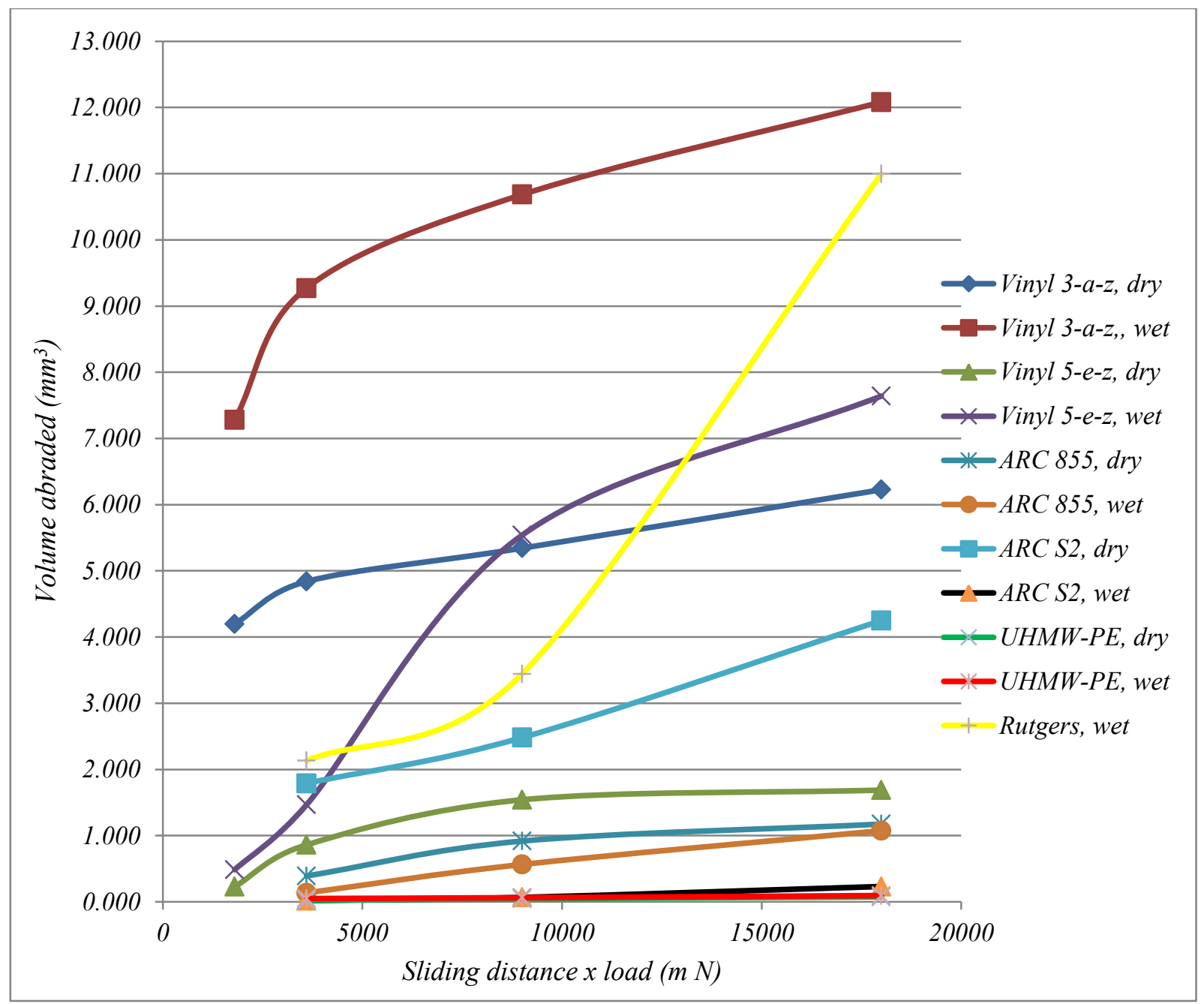

Figure 6.17: Abraded volume vs. sliding distance $x$ load for different coatings

Table 6-10: Wear rate of different coatings

\begin{tabular}{|c|c|c|c|c|c|c|c|c|}
\hline \multirow{3}{*}{ Coatings } & \multicolumn{8}{|c|}{ Wear rate $\left(\mathrm{mm}^{3} / \mathrm{mN}\right) \times 10^{-4}$} \\
\hline & \multicolumn{4}{|c|}{ Dry surface } & \multicolumn{4}{|c|}{ Wet surface } \\
\hline & $<\mathbf{5 k}$ & $5 \mathrm{k}-10 \mathrm{k}$ & 10k-25k & $25 \mathrm{k}-50 \mathrm{k}$ & $<5 \mathrm{k}$ & $5 \mathrm{k}-10 \mathrm{k}$ & 10k-25k & 25k-50k \\
\hline Vinyl 3-a-z & 23 & 4 & \multicolumn{2}{|c|}{1} & 40 & 10 & \multicolumn{2}{|c|}{2} \\
\hline Vinyl 5-e-z & \multicolumn{3}{|c|}{7} & 2 & \multicolumn{3}{|c|}{2} & 0.2 \\
\hline ARC 855 & \multicolumn{4}{|c|}{0.5} & \multicolumn{4}{|c|}{0.6} \\
\hline ARC S2 & & 5 & \multicolumn{2}{|c|}{2} & \multicolumn{4}{|c|}{0.5} \\
\hline UHMW-PE & \multicolumn{4}{|c|}{4} & \multicolumn{4}{|c|}{0.06} \\
\hline Flexible polymer & & 39 & & 0 & & 6 & 2 & 8 \\
\hline
\end{tabular}


For Vinyl 5-e-z specimens abraded at dry condition, the wear is less compared to Vinyl 3-a-z coated specimens. The wear rate is more for initial 10k cycles of abrasion and after that it is decreased. While for specimens abraded underwater, the wear is seen more and increasing constantly.

For specimens coated with ARC 855, there is very less wear on the coatings and is at a constant rate under dry surface, while abrasion underwater shows even less wear after 50k cycles of abrasion. The initial higher wear rate is not observed for ARC 855 coated specimens. For specimens coated with ARC S2 abraded at no condition, the initial wear rate is higher for first $5 \mathrm{k}$ cycles of abrasion but after that the wear rate is seen to be constant, while abrasion underwater shows very less wear of the material almost negligible after 50k cycles of abrasion. For UHMWPE material, the abrasion after 50k cycles at both wet and dry surface show almost negligible amount of wear, while specimens coated with Flexible polymer coating shows very high wear when abraded at no conditionings but with abrasion underwater showed less wear compared to its wear under dry surface.

\subsection{Effect of Surface Wettability}

Further, the wettability and contact angles of water droplet on these coatings was evaluated and shown in Figure 6.18 and Table 6-11. Liquid meets the solid surface at specific angle which is referred to as contact angle. The contact angle is specific for any given substance on a particular surface. Specifically, a contact angle $<90^{\circ}$ indicates that wetting of the surface is favorable, and the fluid will spread over a large area on the surface; while contact angles $>90^{\circ}$ generally means that wetting of the surface is unfavorable so the fluid will minimize its contact with the surface and form a compact liquid droplet. A high contact angle indicates a low solid 
surface energy or chemical affinity. This is also referred to as a low degree of wetting. A low contact angle indicates a high solid surface energy or chemical affinity, and a high or sometimes complete degree of wetting. Contact angle for water and glass surface is nearly $0^{\circ}$ which makes glass as perfectly hydrophilic material. Wetting helps to build up a tribo-film that is able to carry the load and separate surfaces [38].

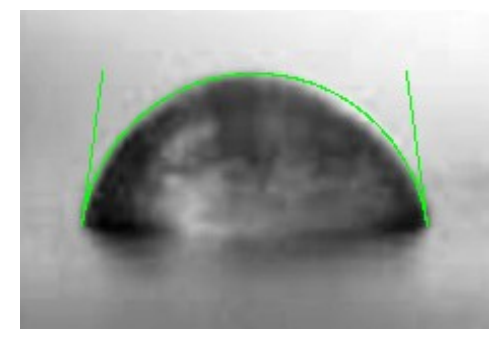

Vinyl 3-a-z

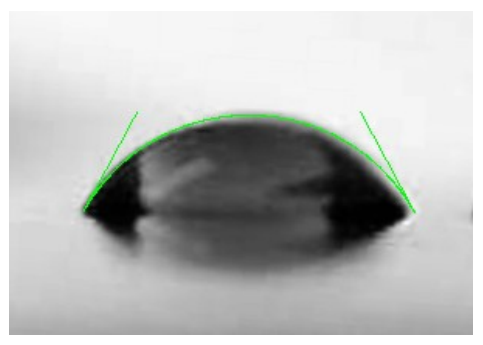

ARC S2

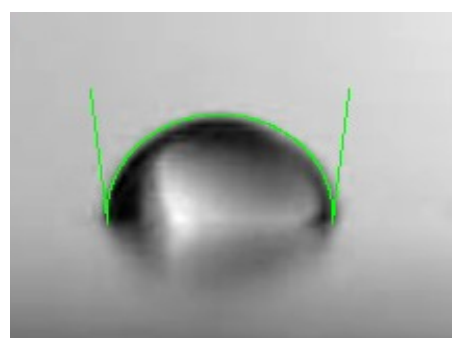

Vinyl 5-e-z

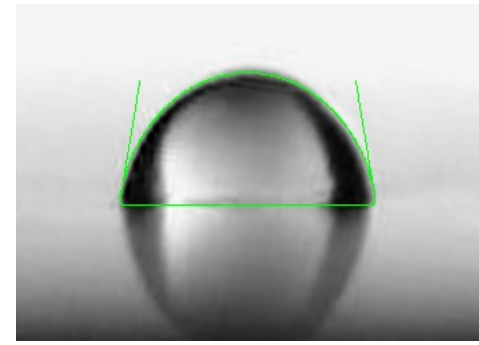

UHMW-PE

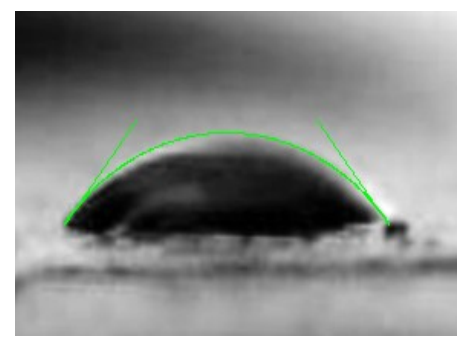

ARC 855

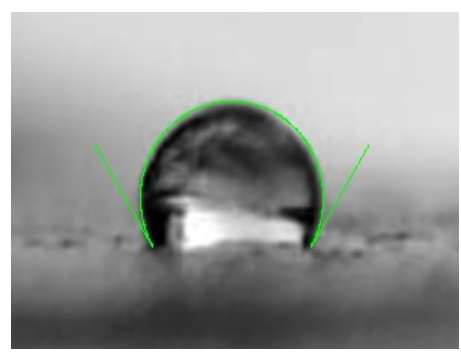

Flexible polymer

Figure 6.18: Water drop and its contact angles on different coating materials

Table 6-11: Contact angles of different coatings

\begin{tabular}{|c|c|}
\hline \multicolumn{1}{|c|}{ Coatings } & Contact angles \\
\hline \hline Vinyl 3-a-z & 82.1 (hydrophilic) \\
\hline \hline Vinyl 5-e-z & 97.2 (hydrophobic) \\
\hline \hline ARC 855 & 48.1 (hydrophilic) \\
\hline \hline ARC S2 & 62.7 (hydrophilic) \\
\hline \hline UHMW-PE & 79.1 (hydrophilic) \\
\hline \hline Flexible polymer & 120.2 (hydrophobic) \\
\hline
\end{tabular}

There is a relationship between contact angle, wetting and interfacial tension. When contact angle increases, wetting decreases and therefore interfacial tension between liquid and solid increases. Material surface with low contact angle is referred to as hydrophobic. 
Considering only the abrasion under wet conditions, material to be used as a coating should be hydrophobic given the abrasive particles are mostly hydrophilic in nature. In that case, the water repelled from the coated surface and water adsorbed by the abrasive particle acts as a cushion and reduces the contact. The very less abrasion of materials during the test with steel ball as an abrader (perfectly hydrophilic) and flexible polymer coating (more hydrophobic) also proves the above statement. Most of the abrasive particles/debris in the flowing water is hydrophilic in nature and thus hydrophobic material is a good choice for coating such gates which are under constant abrasion. However, when the abrasive particles are hydrophobic in nature then there is no any lubricating effect of water.

Considering all four possibilities i) hydrophilic particles - hydrophilic coating, ii) hydrophilic particles - hydrophobic coating, iii) hydrophobic particles - hydrophilic coating, and iv) hydrophobic particles - hydrophobic coating, lubricating effect of water (less friction) is seen in first three cases. Thus, when hydrophilic coating is selected uncertain of what kind of abrasive particles are present, it at least will have an effect of lubrication under water.

\subsection{Conclusions}

Based on the study and experimental results, following conclusions can be stated.

1. Among coatings, ARC 855 and ARC S2 composite coating performed the best under dry and water immersion abrasion testing, respectively.

2. Among vinyl 3-a-z and vinyl 5-e-z, both showed similar depth of abrasion under wet conditions at 50K cycles, whereas vinyl 5-e-z showed better abrasion resistance under dry conditions. However, volume loss was higher for vinyl-3-a-z. 
3. UHMW-PE plate attached to metallic surface showed least amount of abrasion. Coatings and plate attachments with flexible or smooth surface such as Flexible and UHMW-PE performed better under water immersion conditions as compared to their dry surface abrasion because of water lubrication effect.

4. This study has selected and evaluated relative abrasion resistance of several coating systems. Field conditions such as curved dimensions, surface conditions and profiles have to be carefully considered while applying these coating systems.

5. Flexible (fibrous) polymer coating showed the maximum contact angle of $120.2^{0}$, indicating hydrophobic nature and percentage reduction in abrasion from dry to wet condition was the highest for this coating.

6. Wettability is not only the criterion that makes coatings withstand abrasive forces. Many other criteria like roughness (smoothness)/toughness etc. of the surface also affects the rate of wear.

7. When hydrophilic abrader is rubbed against hydrophobic coating, layer of water cushion formed between two surfaces resulting in reduced friction and wear rate. On the other hand, when hydrophilic abrader is rubbed against hydrophilic coatings, a thin layer of water adsorbed on their surfaces provides lubrication and friction reduction.

8. Among different coating systems, ceramic composite based coatings provided better performance over the conventional coatings. 


\section{CHAPTER 7 CONCLUSIONS}

Conclusions of this research for underwater wrapping, rehabilitation of discharge ports and Lake Lynn Bridge, evaluation of different coating systems for tainter gates, and testing on FRP Miter block and FRP recess panel are presented in this chapter.

\subsection{Underwater Wrapping}

Based on the structural performance of concrete cylinders confined with water curable Aquawrap repair system, following conclusions are drawn:

1. The underwater FRP repair system provided good confinement for concrete cylinders which was evidenced as the cylinders with 1,2, 3, and 5 layers of GFRP Aquawrap showed per layer strength increase of $57.4 \%, 49.8 \%, 42.4 \%$, and $51.5 \%$, respectively, giving an average strength increase of $\sim 50 \%$ per layer of wrapping.

2. The maximum stiffness increase after the first layer of wrapping was $51 \%$ and the maximum stiffness further increased by $28 \%$ after the second layer of wrapping. After the third layer of wrapping and so on, percentage increase in stiffness was seen less compared to the $\%$ increase in stiffness achieved in the initial layers of wrapping. This showed an increase in maximum stiffness of wrapped cylinders with 5 layers of wrapping by 2.67 times compared to the non-wrapped ones.

3. Area under load vs. axial deformation curves and stress vs. strain curves for varying number of GFRP wrap layers (1 to 5) showed an increase in ductility and energy absorption. It was observed that the energy absorbed after the first layer of wrapping was $63 \%$ more than the energy absorbed on unwrapped concrete cylinders. Similarly, energy 
absorbed after 2, 3, and 5 layers of wrapping was $105 \%, 192 \%$, and $322 \%$ respectively, more than the energy absorbed on the non-wrapped ones.

4. The experimental confined strength values correlated well within $8 \%$ as per ACI $440.2 \mathrm{R}-$ 08 FRP confinement equation. Further, the predicted confined strength values from different confinement models proposed by different authors resulted similar to the experimental results.

\subsection{Rehabilitation of Concrete Discharge Ports in Chickamauga Dam}

Based on the rehabilitation work conducted in discharge ports at Chickamauga dam, following conclusions are drawn:

1. Aquawrap repair system was field implemented successfully with the help of a team of divers at a depth of $\sim 20 \mathrm{ft}$. in discharge ports of Chickamauga dam, TN. As reported by the diving team, the materials and the bond integrity were visually inspected underwater and found to be excellent, three months after the installation.

2. Water curable FRP repair system (Aquawrap) provided an economical solution for underwater repair of concrete structures. It is noted that the surface preparation of the submerged concrete structures is important to get better bonding between FRP system and the substrate.

\subsection{Rehabilitation of Steel Piles in East Lynn Lake Bridge}

Based on the rehabilitation work conducted on East Lynn Lake Bridge piles, following conclusions are drawn: 
1. Concrete cylinders with steel section and wrapped with 2 layers of Aquawrap showed significant increase in load capacity with degree of compositeness being greater than $92 \%$.

2. The rehabilitation scheme resulted in enhancing the column failure load capacity by $\sim 2.5$ times that of yield capacity of 12 BP 53 section. Despite rehabilitation and increased load capacity, the steel columns will only be rated up to their original capacity to yield which is 558 kips per column of $36 \mathrm{ksi}$ steel.

3. Weight limit after successful rehabilitation has been upgraded and increased from 6 tons to 30 tons.

4. The concrete and the FRP composites (jackets and wraps) that encase the corroded steel provide three layers of protection against future corrosion and give superior strength (corrosion probes have been installed in the site for studying the future corrosion rate).

5. The use of FRP composites for rehabilitation provided cost and time advantage (cost is about $2 / 3$ less than conventional construction and time for the completion of the rehabilitation is about 3 weeks) including reduction in future maintenance costs and enhanced durability.

6. The temperature-humidity levels influence the material curing properties and site work progress and require the work-plan to accommodate those variations.

\subsection{Abrasion Resistant Coatings}

Based on the study and experimental results, following conclusions are drawn:

1. Among coatings, ARC 855 and ARC S2 composite coating performed the best under dry and water immersion abrasion testing, respectively. 
2. Among vinyl 3-a-z and vinyl 5-e-z, both showed similar depth of abrasion under wet conditions at 50K cycles, whereas vinyl 5-e-z showed better abrasion resistance under dry conditions. However, volume loss was higher for vinyl-3-a-z.

3. UHMW-PE plate attached to metallic surface showed least amount of abrasion. Coatings and plate attachments with flexible or smooth surface such as Flexible and UHMW-PE performed better under water immersion conditions as compared to their dry surface abrasion because of water lubrication effect.

4. This study has selected and evaluated relative abrasion resistance of several coating systems. Field conditions such as curved dimensions, surface conditions and profiles have to be carefully considered while applying these coating systems.

5. Flexible (fibrous) polymer coating showed the maximum contact angle of $120.2^{0}$, indicating hydrophobic nature and percentage reduction in abrasion from dry to wet condition was the highest for this coating.

6. Wettability is not only the criterion that makes coatings withstand abrasive forces. Many other criteria like roughness (smoothness)/toughness etc. of the surface also affects the rate of wear.

7. When hydrophilic abrader is rubbed against hydrophobic coating, layer of water cushion formed between two surfaces resulting in reduced friction and wear rate. On the other hand, when hydrophilic abrader is rubbed against hydrophilic coatings, a thin layer of water adsorbed on their surfaces provides lubrication and friction reduction.

8. Among different coating systems, ceramic composite based coatings provided better performance over the conventional coatings. 


\subsection{FRP Miter Block and FRP Recess Panel}

Based on the testing on FRP miter blocks and FRP recess panel, as described in Appendices A and B, following conclusions are drawn:

1. Thermal expansion coefficients of the miter block with 2 stage of manufacturing indicates different in three directions.

2. Differential Scanning Calorimetry (DSC) tests indicate different degree of cure within two parts of the block that were independently manufactured but thermally fused.

3. Based on thermal coefficients and different degrees of cure within a block, it is decided to manufacture the FRP miter block in a single stage than two stages of fusing two blocks with unequal thicknesses.

4. The recess panel made with twelve FRP superdecks bonded with shear connecters and "Rhino liner" coating was able to distribute the load effectively throughout the panel.

5. The load applied on a single beam was effectively distributed to several other beams increasing the amount of energy absorption and the spreading of impact forces.

6. The failure load for the single beam of the panel directly under the applied load is estimated to be approximately 4.5 times greater than its individual capacity. 


\section{REFERENCES}

[1] "Tennessee Department of Transportation, Structures Division - Hydraulic Design Section," [Online]. Available:

http://www.tdot.state.tn.us/chief_engineer/assistant_engineer_design/structures/hydraulc.htm

[2] "America's Infrastructure," March 2013. [Online]. Available:

http://www.infrastructurereportcard.org/a/browser-options/downloads/2013-Report-

Card.pdf.

[3] R. Sen and G. Mullins, "Application of FRP composites for underwater piles repair," Composites Part B:engineering, pp. 751-758, 2007.

[4] "Bridge Structural Elements Diagram," [Online]. Available: http://www.michigan.gov/mdot/0,4616,7-151-9618_47418-173584--,00.html.

[5] R. Lampo, "Polymer Composite Materials for the Repair and Maintenance of Navigation Structures," US Army Corps of Engineers, Champaign, Illinois, 2013.

[6] L. Lam and J. G. Teng, "Design-oriented stress-strain model for FRP-confined concrete," Construction and Building Materials, vol. 17, no. 6-7, pp. 471-489, 2003.

[7] R. R. Rajappa, "Behavior of FRP Wrapped Concrete Cylinders," West Virginia University, 2004.

[8] S. Bazinet, L. Cercone and F. Worth, "Innovative FRP Piling Repair Without the Use of Coffer Dams," in 48th International SAMPE J, California, 2003.

[9] G. Mullins, R. Sen, K. S. Suh and D. Winters, "Underwater FRP Repair of Prestressed Piles in the Allen Creek Bridge," Journal of Composites for Construction, ASCE, vol. 9, no. 2, pp. 
136-146, 2005.

[10] A. L. Corporation, "Field- Applied Composite Systems- Aquawrap," [Online]. Available: http://www.airlog.com/FACS/FACS\%20Aquawrap.htm.

[11] "Emergency Bridge Column Repair," [Online]. Available: http://www.airlog.com/FACS/FACS\%20Aquawrap\%20Concrete\%20Column.htm.

[12] C. Alexander, "Evaluation of the Aquawrap System in Repairing Mechanically Damaged Piles," Stress Engineering Services, Inc., California.

[13] D. Winters, G. Mullins, R. Sen, A. Schrader and M. Stokes, "Bond Enhancement for FRP Pile Repair in Tidal Waters," Journal of Composites for Construction, vol. 12, no. 3, pp. 334-343, 2008.

[14] A. Chalid, J. M. Yatim and A. K. Wahab, "Performance of Epoxy Putty FRP for Underwater Structural Concrete," International Journal of Civil, Structural, Environmental and Infrastructure Research and Development, pp. 33-44, 2012.

[15] I. M. Hutchings, Tribology, Friction and Wear of Engineering Materials, 1992.

[16] E. Rabinowicz and A. Mutis, "Effect of Abrasive Particle Size on Wear," Wear, vol. 8, no. 5, pp. 383-390, 1965.

[17] I. Kovariova, B. Szewczykova, P. Blaskovits, E. Hondulova and E. Lechovic, "Study and Characteristic of Abrasive Wear Mechanisms".

[18] J. A. Hawk and R. D. Wilson, "Abrasive Wear Failures," Albany Research Center.

[19] Z. Gahr and K. Heinz, Microstructure and wear of materials, Tribology 10, Elsevier science publishing company inc., 1987.

[20] Z. Gahr and K. Heinz, "Modeling of two-body abrasive wear," in Wear, 1988, pp. 87-103. 
[21] K. Z. Gahr, "Wear by hard particles," Tribology International, vol. 31, no. 10, pp. 587-596, 1998.

[22] A. Borruto, G. Crivellone and F. Marani, "Influence of Surface Wettability on Friction and Wear Tests," Wear , vol. 222, pp. 57-65, 1998.

[23] D. A. Barrow, T. E. Petroff and M. Sayer, "Thick Ceramic Coating using a Sol Gel based Ceramic- Ceramic 0-3 Composite," Surface and Coatings Technology, 1995.

[24] "Engineering Materials - Ceramics," [Online]. Available: http://www.engineershandbook.com/Materials/ceramics.htm.

[25] "Innovative Engineered Coating Solutions," Thermal Spray Technologies, Inc., [Online]. Available: http://www.tstcoatings.com/wear_resistant_coatings.html.

[26] A. 1. corporation, "Product technical data sheet- Aquawrap Primer/Adhesive Systems".

[27] S. Matthys, H. Toutanji and L. Taerwe, "Stress-Strain Behavior of Large-Scale Circular Columns Confined with FRP Composites," Journal of Structural Engineering, vol. 132, no. 1, pp. 123-133, 2006.

[28] J. Mander, M. Priestley and R. Park, "Theoretical Stress-Strain Model for Confined Concrete," Journal of Structural Engineering, vol. 114, no. 8, pp. 1804-1826, 1988.

[29] H. Toutanji, "Stress-Strain Characteristics of Concrete Columns Externally Confined with Advanced Fiber Composite Sheets," American Concrete Institute , vol. 96, no. 3, pp. 397404, 1999.

[30] "Guide for the Design and Construction of Externally Bonded FRP Systems for Strengthening Concrete Structures," ACI Committee 440, 2008.

[31] U. A. C. o. Engineers, "Chickamauga Lock Replacement," [Online]. Available: 
http://www.lrn.usace.army.mil/Media/FactSheets/FactSheetArticleView/tabid/6992/Article/ 6592/chickamauga-lock-replacement.aspx.

[32] F. Tucker, "Nashville District works with partners on engineering solutions at Chickamauga Lock," 29 August 2013. [Online]. Available: http://www.Irn.usace.army.mil/Media/NewsStories/tabid/6957/Article/17796/nashvilledistrict-works-with-partners-on-engineering-solutions-at-chickamauga.aspx.

[33] K. Kemp, "WVU students bridge infrastructure gap," 29 March 2014. [Online]. Available: http://www.wvgazette.com/News/201403290062.

[34] J. A. Williams, "Engineering Tribology," Oxford, Oxford University Press, 1994, p. 167.

[35] K. L. Rutherford and I. M. Hutchings, "A micro-abrasive wear test with particular application to coated systems," Surface coat and technol, vol. 79, pp. 231-239, 1996.

[36] I. M. Hutchings, "Abrasive and erosive wear tests for thin coatings: a unified approach," Tribology International, vol. 31, pp. 5-15, 1998.

[37] E. Rabinowicz, Friction and Wear of Materials, NY: John Wiley, 1964.

[38] Z. Pawlak, W. Urbaniak and A. Oloyede, "The relationship between friction and wettability in aqueous environment," Wear, vol. 271, pp. 1745-1749, 2011.

[39] T. Beaty, "FRP Composites for Lock and Dam Structures," WVU, Morgantown, 2013.

[40] B. S. Yigezu, P. K. Jha and M. M. Mahapatra, "Effect od Sliding Distance, Applied Load and Weight Percentage of Reinforcement on the Abrasive Wear Properties of In Situ Syntesized Al-12\%/TiC Composites," Tribology Transactions, vol. 56, no. 4, pp. 546-554, 2013. 


\section{APPENDIX A: Application of FRP Wraps and Coatings}

Table A-1: Steps for field wrapping of columns

\begin{tabular}{|c|c|c|}
\hline Steps & Details/Procedures & \\
\hline .. & $\begin{array}{l}\text { Use appropriate underwater suits/gear and Tyvek suit covers. } \\
\text { Use pressurized water jet cleaning method or powered hand tools } \\
\text { operated at up to } 4500 \mathrm{rpm} \text { with rotary cleaning attachments (steel } \\
\text { brushes or abrasive } 80-120 \text { grit sanding discs, e.g. Stanley hydraulic } \\
\text { grinder) to clean the surface of concrete to make it free from } \\
\text { oil/grease, dirt, loose materials, calcareous debris, and spalling } \\
\text { concrete. } \\
\text { Use underwater curable grout to fill up any cavities \& cracks. } \\
\text { Smooth the entire concrete surface by sanding \& abrading such that it } \\
\text { is free from protrusions and cavities. }\end{array}$ & $\begin{array}{l}\text { Steel Disc brush } \\
\text { grinding discs }\end{array}$ \\
\hline 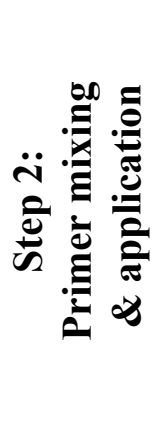 & $\begin{array}{l}\text { Pour contents of BP-4 part B into BP-4 part A in the manufactured } \\
\text { recommended proportion of } 1: 2 \text {. (Max. container size is } 1 / 2 \text { gallon) } \\
\text { Mix BP-4 Primer part-A and BP-4 Primer part-B with a } \\
\text { mechanical mixer for about } 3 \text { minutes at low speeds }(500-1000 \mathrm{rpm}) \text {. } \\
\text { Application temp. is } 55-120^{0} \mathrm{~F} \text { with pot life of } 55-75 \text { minutes at } 75^{\circ} \mathrm{F} \text {. } \\
\text { Apply the mixed primer for a min. thickness of } 40 \pm 5 \text { mils all around } \\
\text { concrete surface with a painter's mitt or spreader/trowel/roller. }\end{array}$ & \\
\hline लि & $\begin{array}{l}\text { Open the Aquawrap pouch such that } 1^{\text {st }} \text { layer of wrapping gets } \\
\text { completed within the pot life of primer having max of } 75 \text { minutes at } \\
75^{\circ} \mathrm{F} \text {. } \\
\text { Application temp. of Aquawrap is } 40-200^{0} \mathrm{~F} \text { with gel time of } 30-60 \\
\text { minutes at } 77^{0} \mathrm{~F} \text {. } \\
\text { Wrap the Aquawrap around the concrete by creating tension in the } \\
\text { fabric by hand-pulling or using supplied Tiger tabs. } \\
\text { Wrap until the desired number of layers is reached and trim the excess } \\
\text { fabric and attach it to the composite concrete with supplied Tiger tabs. }\end{array}$ & $S$ \\
\hline 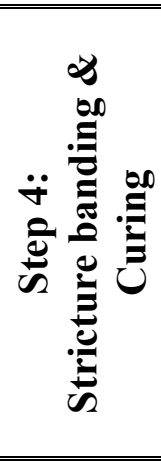 & $\begin{array}{l}\text { Apply } 2 / 4 \text { layers of stricture banding over the Aquawrap } \\
\text { application. } \\
\text { Perforate the stricture banding with pointed perforator tool. } \\
\text { After } 6 \text { hours, remove stricture banding \& apply a final coating of } \\
\text { BP-4 primer on the partially cured FRP wrap \& finish the coating } \\
\text { with a trowel to cover poke holes. } \\
\text { Leave wrapped concrete to cure for about } \mathbf{2 4} \text { to } \mathbf{4 8} \text { hours underwater } \\
\text { Use acetone based solvents for cleaning the resin. }\end{array}$ & $\begin{array}{r}\text { Stri } \\
\text { band } \\
\text { con }\end{array}$ \\
\hline
\end{tabular}


Table A-2: Steps for application of ARC 855 and ARC S2 Coating

\begin{tabular}{|c|c|}
\hline Steps & Details/Procedures \\
\hline 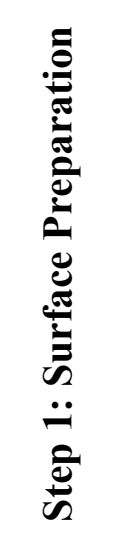 & $\begin{array}{l}\text { Remove rough scales present on steel surface by mechanical means such as scrapers, } \\
\text { high pressurized water, sweep and blast. } \\
\text { Perform abrasive grit blasting to a cleanliness of near white metal (SSPC-SP10) \& } \\
\text { make sure the surface is cleaned of all contaminants and roughened to an angular } \\
\text { profile between } 3 \text { to } 5 \text { mils. } \\
\text { Once performing abrasive blasting, verify the cleanliness as per SSPC Vis } 1 \text { and the } \\
\text { angular profile as per NACE RP } 0287-95 \text {. } \\
\text { After verifying, blow or wipe down the surface to remove blast residues. Apply clear } \\
\text { plastic tape to blasted steel surface and then remove the plastic tape and press it } \\
\text { against a white paper to verify or make sure no blast residue is present. (Note: blast } \\
\text { residues can inhibit adhesion of coating to steel) }\end{array}$ \\
\hline 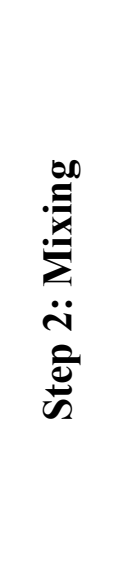 & 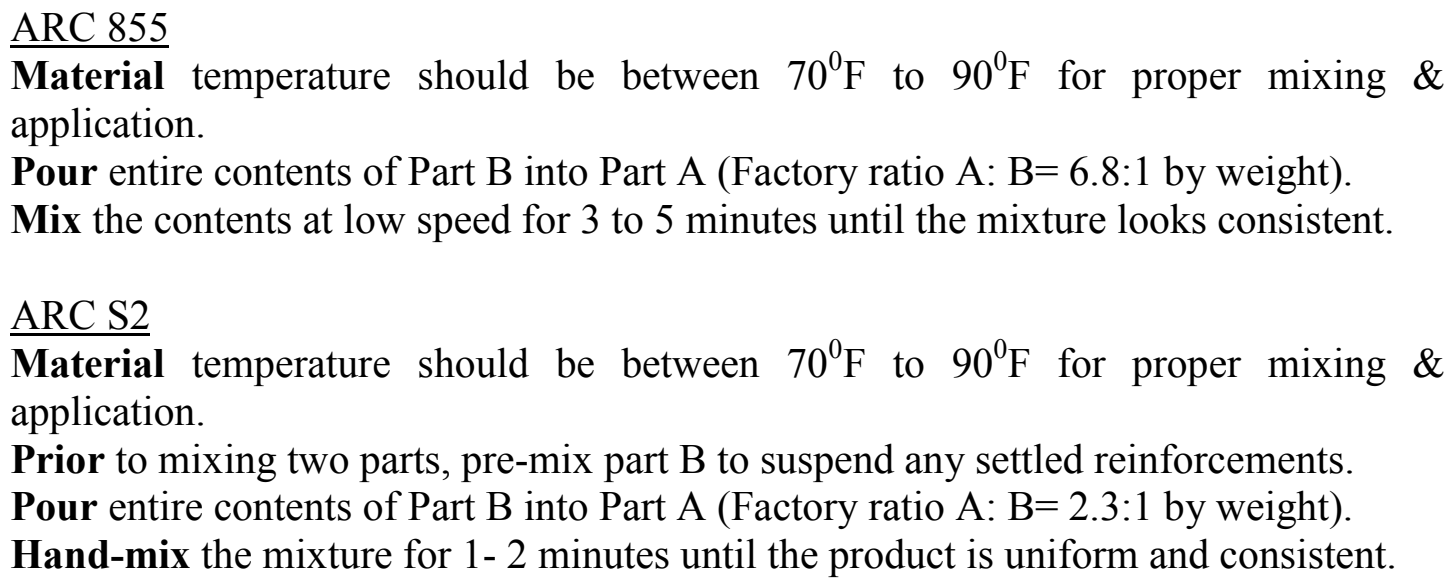 \\
\hline 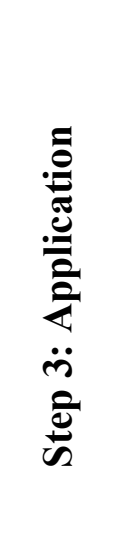 & 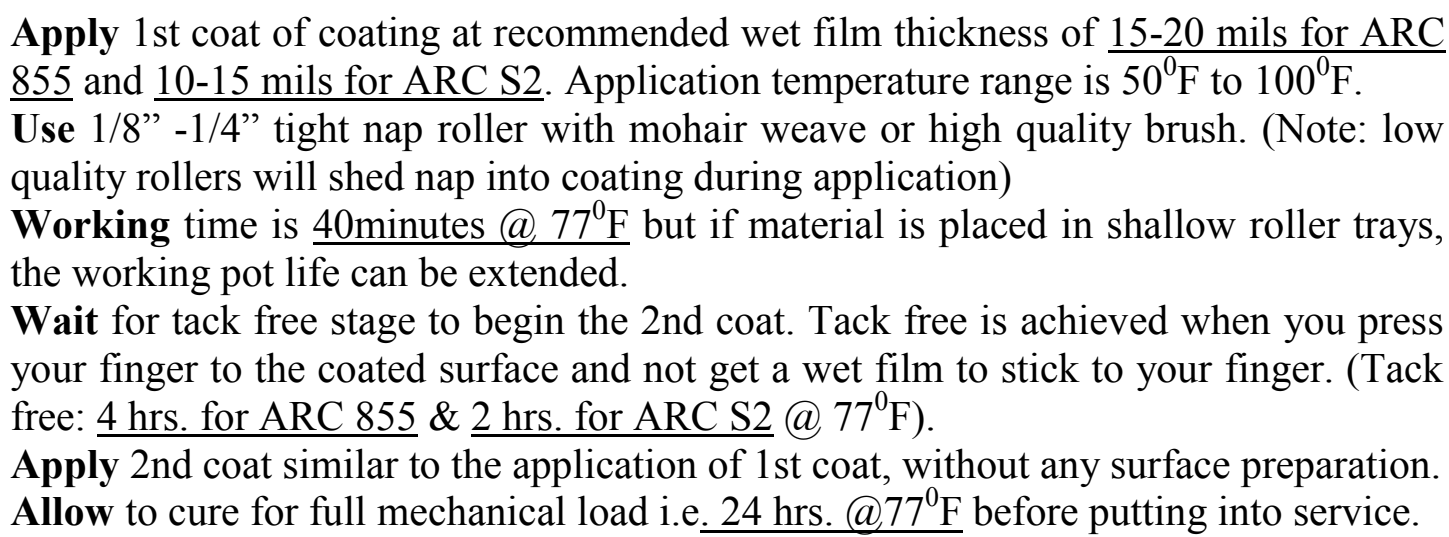 \\
\hline
\end{tabular}




\section{APPENDIX B: FRP Miter Blocks}

The following sections give a brief introduction to FRP miter block and also provide their test results on bending, resin cure, and thermal expansion coefficient. Additional details regarding the FRP miter blocks have been provided in the report "FRP Composites for Lock and Dam Structures" [39].

\section{B.1 Introduction}

Miter blocks are used in hydraulic gates to create a continuous vertical line of contact between two closed miter gate leaves and are exposed to harsh service conditions including wetdry cycles, corrosive elements, hydrodynamic forces, mitering forces, and freeze-thaw effects. Miter blocks currently in service are made with steel. These blocks are heavy and corrode in a short time including the requirement of regular corrosion resistant coatings. Thus, FRP miter blocks were studied and evaluated as the replacement to these steel miter blocks. Based on different configurations evaluated, the single block of FRP miter block (Fig.B.1) was produced by Fiber-Tech, Inc. was selected as the potential FRP miter block. In this work, thermal characteristics of the miter block are evaluated. Purpose of this evaluation is to understand and refine design and manufacturing of FRP blocks to minimize thermal deformations.

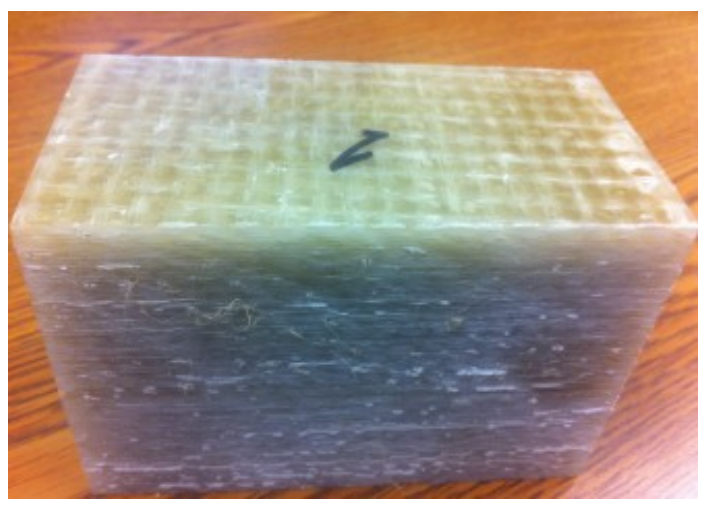

Figure B.1: FRP miter block 


\section{B.2 Bending test on miter block}

Miter block was cut to a length of 23 inches and was placed on roller supports with center to center distance of 18 inches (Fig. B.2). Concentrated load was applied with a steel roller at the center of the block. The load vs. deflection and bending stress vs. strain plots are shown in Fig. B.3. The maximum bending stress was obtained to be 21.4 ksi and the maximum deflection that was recorded at the maximum load was 0.44 inches.
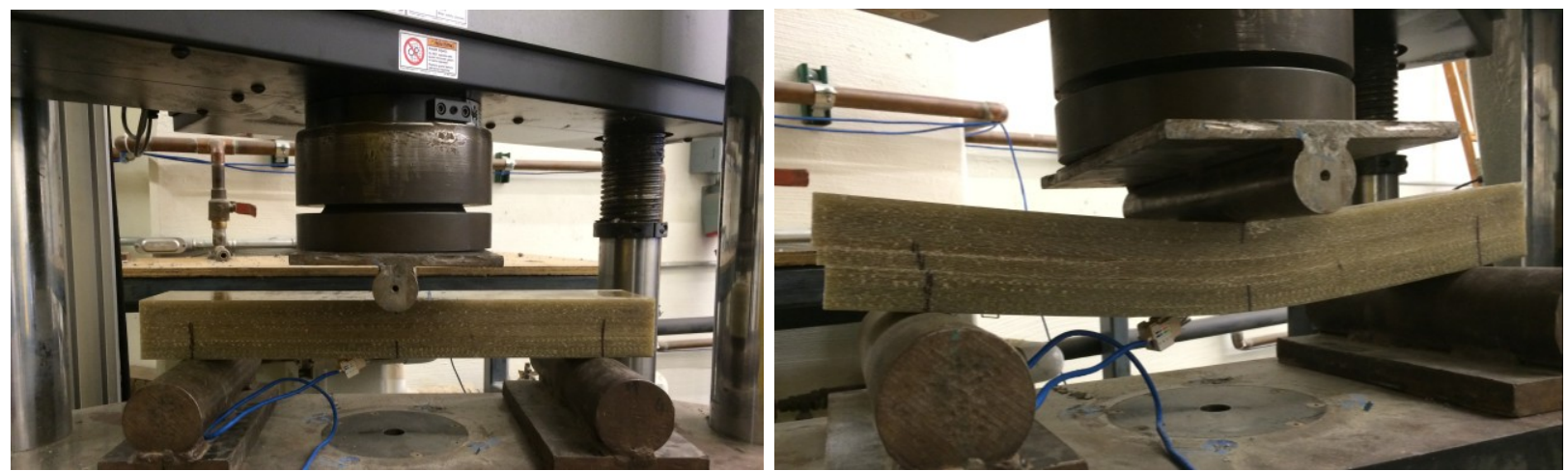

Figure B.2: Bending test on FRP miter block

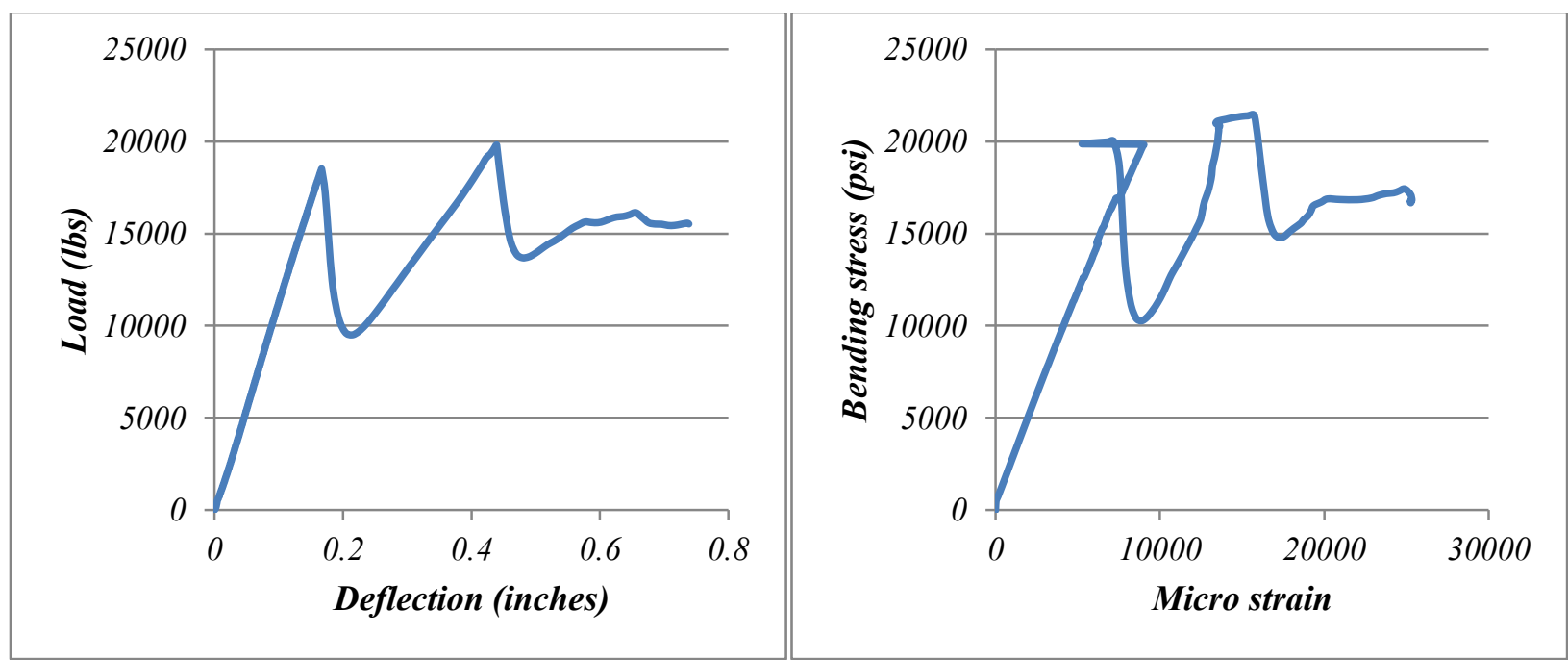

Figure B.3: Load vs. deflection plot and bending stress vs. strain plot 


\section{B.3 DSC test of resin from two surfaces of miter block}

In order to investigate the potential effect of sample surface side on cure percent, differential scanning calorimetry (DSC) tests were conducted which is a thermal analysis technique that measures the temperature and heat flow associated with transitions in materials as a function of time and temperature. Such measurements provide qualitative and quantitative information about physical and chemical changes that involve endothermic or exothermic processes or changes in heat capacity. In addition to identifying glass transition temperatures of these infused laminates, it is intended to identify the rate of cure or degree of cure for two parts of the block that were independently manufactures and thermally fused together.

Two tests were conducted on DSC Q Series Model Q100, TA Instruments Inc. Each test was ramp-heated up to $250^{\circ} \mathrm{C}$ in two cycles. Heat vs. temperature data were recorded for each sample as shown in Figure B.4. As seen from test results, the specimen cut from 1 inch side has higher uncured resin than that of the 1.5 inch side (Fig. B.4).
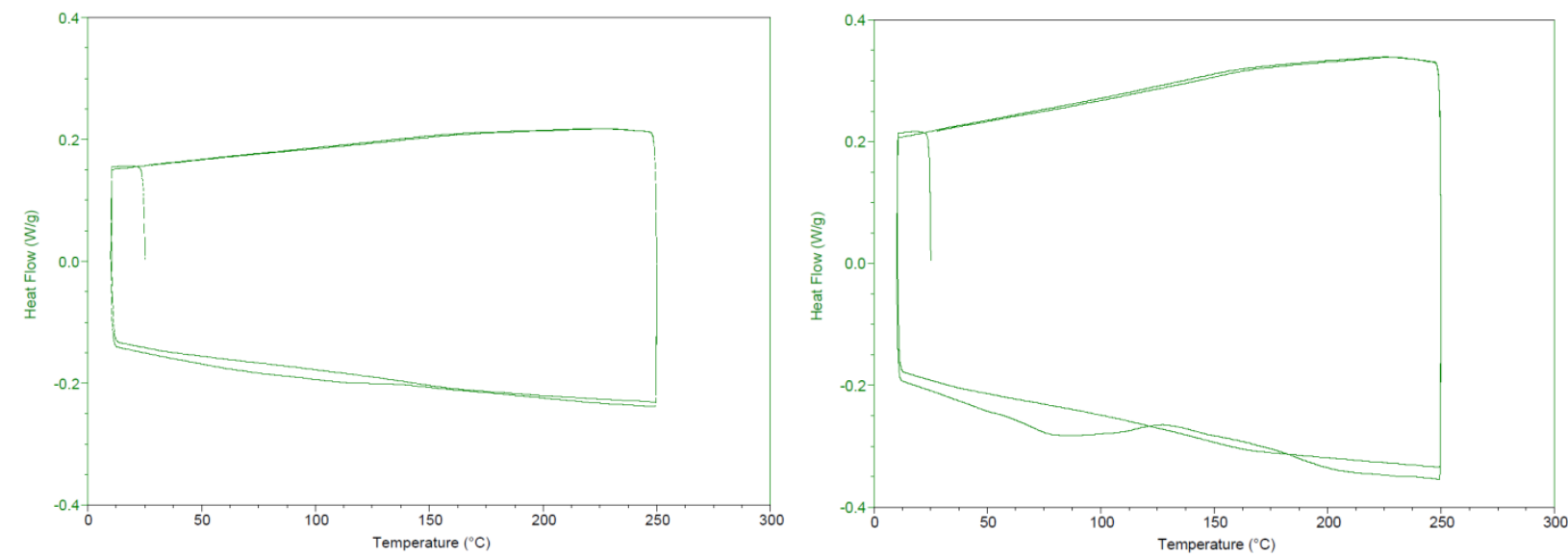

Figure B.4: Resin cure from 1.5 inch side and resin cure from 1 inch side 


\section{B.4 Thermal expansion coefficient of the miter block}

Seven strain gages were placed on different surfaces of 21 " long FRP miter block as shown in Figure B.5. The miter block was vertically placed in the thermal chamber as shown in Figure B.5 and the temperature was varied from the room temperature $\left(69^{\circ} \mathrm{F}\right)$ to $90{ }^{\circ} \mathrm{F}$ and then to $110{ }^{\circ} \mathrm{F}$ and further to $160{ }^{\circ} \mathrm{F}$. For each temperature, miter block was kept in the chamber for enough duration so that the temperature stabilization was uniform throughout the thickness of the block. Table B-1 shows the strain gage readings on miter block in three different directions and Table B-2 shows the computed thermal coefficients.

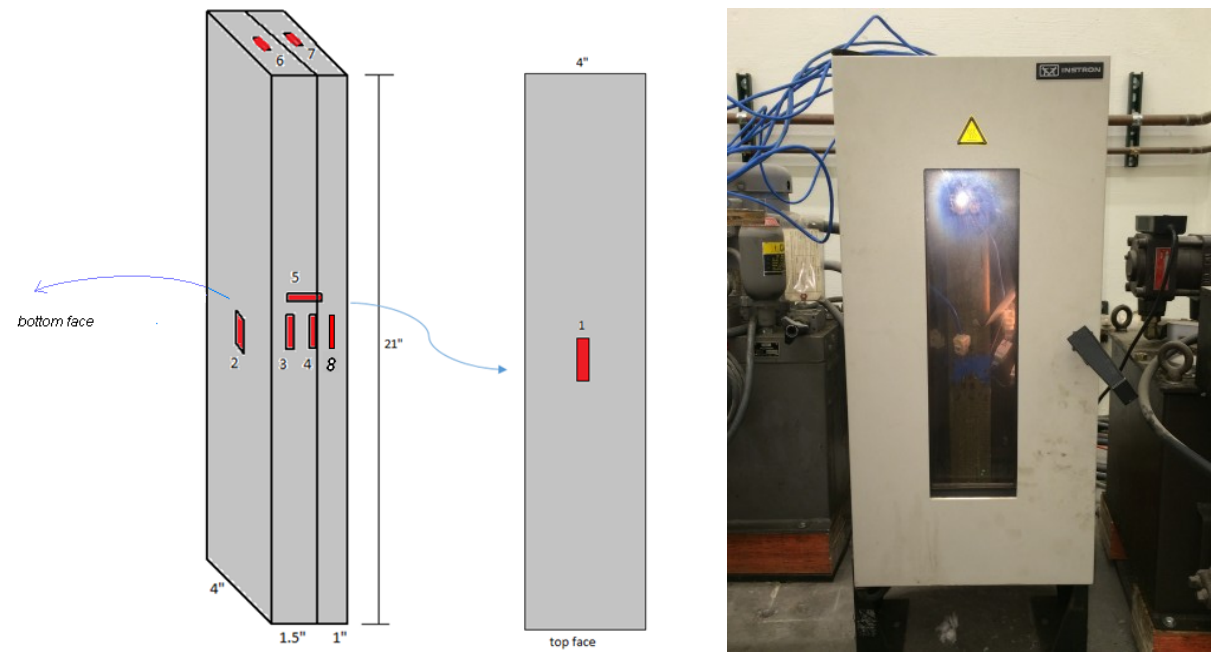

Figure B.5: Strain gages on 21 " long miter block \& temperature controlled chamber

Table B-1: Strain readings of different gages attached to the miter block

\begin{tabular}{|c|c|c|c|}
\hline \multirow{2}{*}{$\begin{array}{c}\text { Strain gage } \\
\text { numbering }\end{array}$} & \multicolumn{3}{|c|}{ Strain $\times 10^{-6}$} \\
\cline { 2 - 4 } & Room temp $=69^{\circ} \mathrm{F}$ & Room temp $=67^{\circ} \mathrm{F}$ & Room temp $=69^{\circ} \mathrm{F}$ \\
\hline 1 & $@ 90^{\circ} \mathrm{F}$ & $@ 110^{\circ} \mathrm{F}$ & $@ 160^{\circ} \mathrm{F}$ \\
\hline 2 & 183 & 311 & 648 \\
\hline 3 & 173 & 353 & 685 \\
\hline 4 & 109 & 368 & 614 \\
\hline 5 & 102 & 371 & 622 \\
\hline 6 & 735 & 1753 & 3772 \\
\hline 7 & 151 & 367 & 706 \\
\hline
\end{tabular}


Table B-2: Thermal coefficients for the miter block in different direction

\begin{tabular}{|c|c|c|}
\hline Direction & Gage number & $\begin{array}{c}\text { Thermal coefficients } \times 10^{-6} \\
\left.\text { (in/in/ } /{ }^{\circ} \mathrm{F}\right)\end{array}$ \\
\hline $\begin{array}{c}\text { Longitudinal } \\
\text { (Gate height direction) }\end{array}$ & $2(1$ " side $)$ & 7.12 \\
\hline $\begin{array}{c}\text { Depth direction } \\
\text { (2.5" dimension) }\end{array}$ & 5 & 7.52 \\
\hline $\begin{array}{c}\text { Width direction } \\
\text { (4" dimension) }\end{array}$ & 6 & 41.45 \\
\cline { 2 - 3 } & 7 & 7.76 \\
\hline
\end{tabular}

\section{B.5 Conclusions}

1. Thermal expansion coefficients of the miter block with 2 stage of manufacturing indicates different in three directions.

2. Differential Scanning Calorimetry (DSC) tests indicate different degree of cure within two parts of the block that were independently manufactured but thermally fused.

3. Based on thermal coefficients and different degrees of cure within a block, it is decided to manufacture the FRP miter block in a single stage than two stages of fusing two blocks with unequal thicknesses. 


\section{APPENDIX C: FRP Recess Panels}

\section{C.1 Introduction}

Recess panels are typically located in the upper lock approach and are used in many navigational structures and are used to protect the recess areas in the lock chamber from impact damage to lock walls. Figure C.1 shows a recess panel from the upper lock approach at Willow Island lock and dam in Ohio River. Recess panels currently in service are manufactured using steel I-beams, angles, and flat sheets. These panels are heavy to lift and corrode in a short time including the requirement of regular corrosion resistant surface coatings. Thus, FRP recess panels were studied and evaluated as the replacement to these steel recess panels.

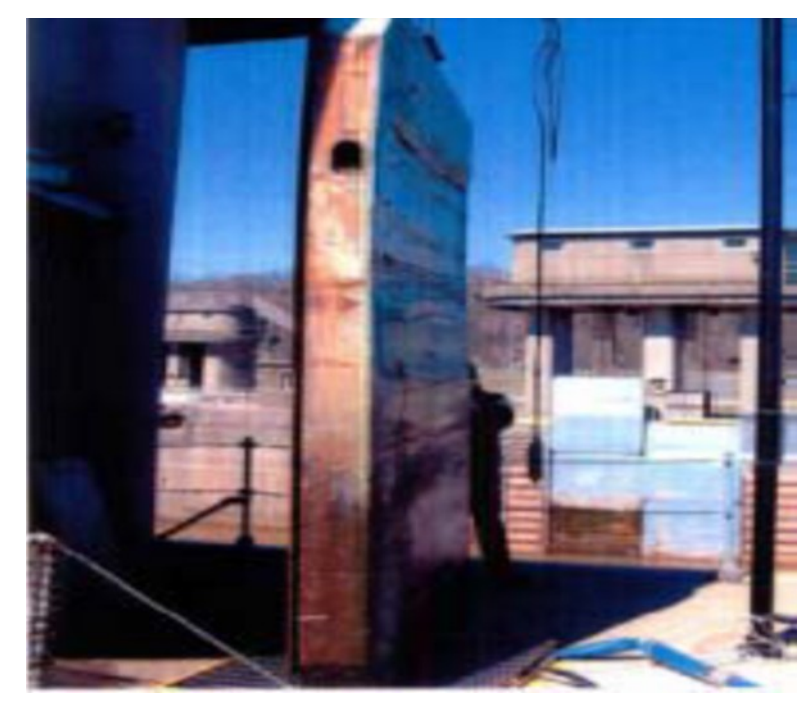

\section{Figure C.1: Steel recess panel subjected to corrosion and abrasion}

Based on 4 different configurations evaluated, hexagonal deck was the best FRP shape in terms of energy absorption for implementation as recess protection panel. Crushing failure and moment capacity of the single hexagonal FRP superdeck at a clear span of 96 inches was 48.4 lbs and $96.8 \mathrm{ft}-\mathrm{kip}$, respectively. In field conditions, the panel has to withstand sudden barge hit and the resulting impact load is distributed to one or several beams of the FRP panel system. 


\section{C.2 Objective}

The main objective of this testing was to investigate the degree of load transfer across the hexagonal sections that were adhesively bonded together as a single panel. Hence, discussion on analysis of additional data generated during this testing is not included in this section.

\section{C.3 Test methods}

The final dimension of the assembled (finished) FRP recess panel was 9' $10.5^{\prime \prime}$ wide by 11' 10" tall. The recess panel made with 12 FRP superdecks (Figures. C.2 \& C.3) was brought and tested under different loading conditions at WVU structures lab. In order to determine load distribution of FRP beams, panel was tested in bending at span of $8 \mathrm{ft}$. with application of load directly at the center beam (seventh FRP superdeck beam, Figure C.3) with three contact areas utilized for the load application: i) 6 in $\times 6$ in, ii) 6 in $x 24$ in, and iii) 6 in $x 48$ in. The load was applied horizontally as well as vertically as shown in the Figure C.3. Strains, deflections, and distribution of the applied load across each hexagonal unit at specific locations were measured and studied. Figure C.2 shows a single hexagonal FRP superdeck beam with a shear connector and top coating referred to as Rhino liner. Figure C.4 shows the test panel with strain and deflection gages positioned at different locations. Additional details regarding FRP recess panels have been provided in the report "FRP Composites for Lock and Dam Structures" [6].

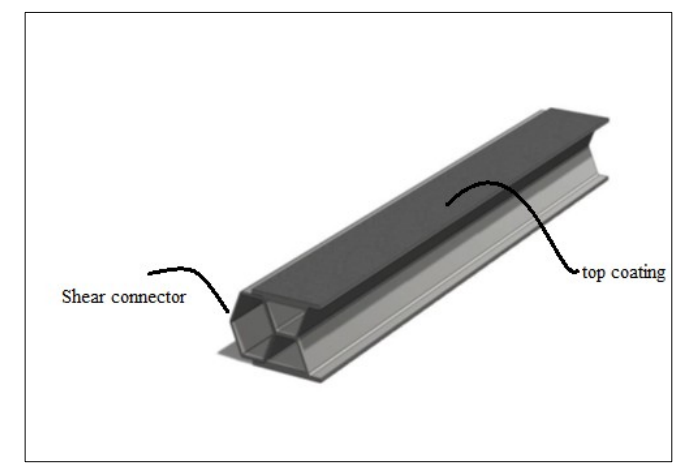

Figure C.2: Hexagonal superdeck with shear connectors and top coating 

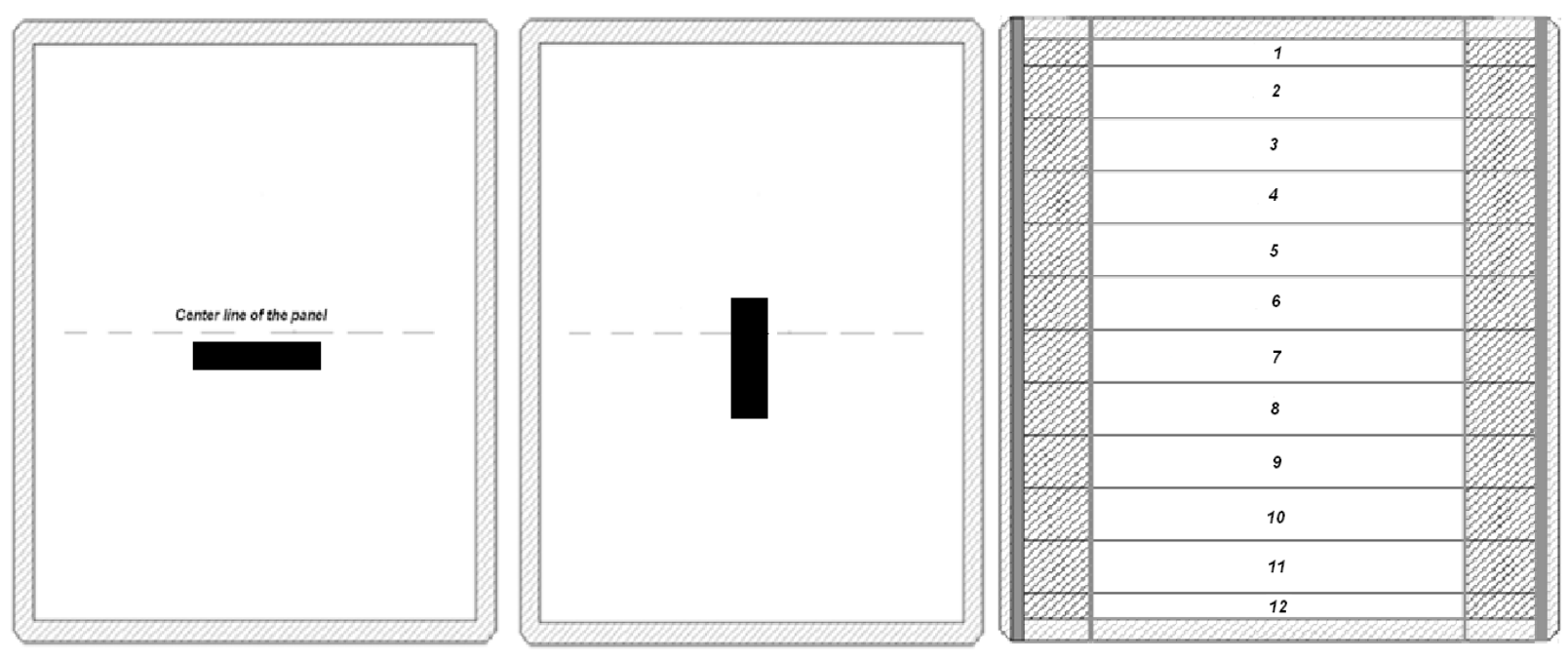

Figure C.3: Load application areas- horizontal (left), -vertical (middle), beam numbering of panel (right)

NOTES: Horizontal and vertical orientations are with respect to the panel placement in the lock and dam structure.

Load application areas shown as rectangles had dimensions of 6in x 6in, 6in x 24in, and 6in x 48in.

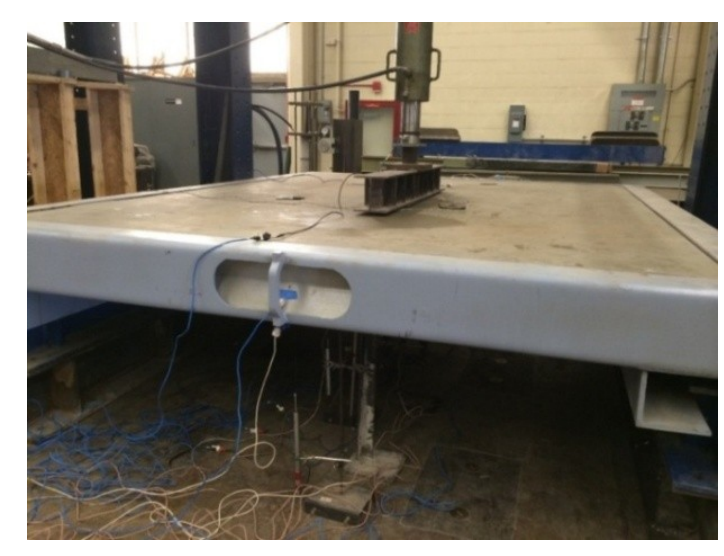

Figure C.4: Recess panel testing

\section{C.4 Test results}

The test results have been presented in two sub headings (C.4.1 and C.4.2) based on the variations in the measurement of deflections discussed in this section.

\section{C.4.1 Deflections measured in both horizontal and vertical directions of the panel (Fig. C.3)}

In this testing, deflections were measured in both directions of the panel as shown in Figure C.6. Strain gage locations as well as LVDT locations on top, front, side and bottom face 
of the panel is shown in Figure C.5 and C.6. All the locations have been numbered for consistency and ease of test result presentations. The rectangle represents the strain gage whereas the circle represents the LVDTs. The test results shown within this section correspond to the locations of strain gages and LVDTs. Seventh beam (Figure C.3) has been referred to as center beam throughout all the sections in this report. The following testing has been done with deflections being measured in both horizontal and vertical directions.

i. $\quad$ With 6 in $x 6$ in plate placed at the center beam (Fig. C.3)

ii. With 6 in $x 24$ in plate placed horizontally at the center beam (Fig. C.3)

iii. $\quad$ With 6 in $x 24$ in plate placed vertically at the center beam (Fig. C.3)

iv. With 6 in $x 48$ in plate placed horizontally at the center beam (Fig. C.3)

v. With 6 in $x 48$ in plate placed vertically at the center beam (Fig. C.3)

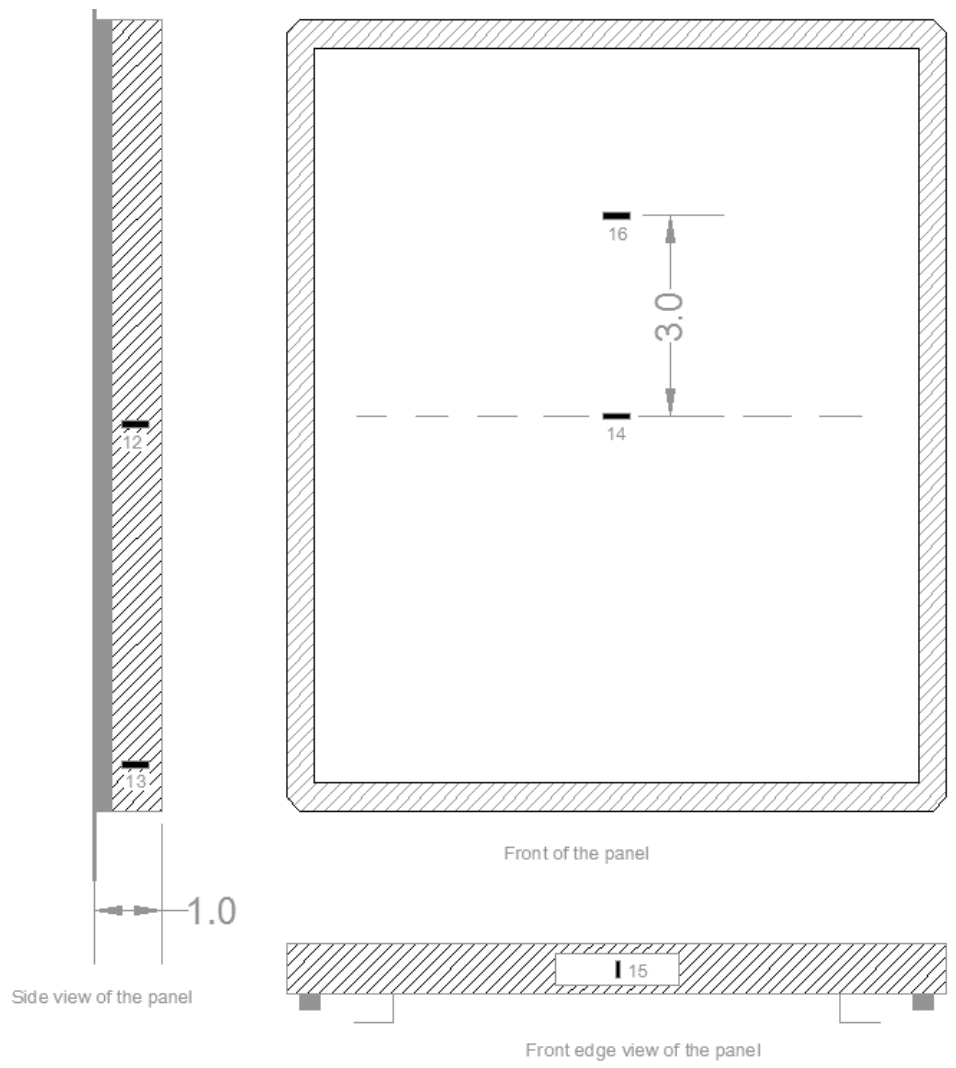

Figure C.5: Locations of strain gage (rectangle) on top, front and side of the panel 


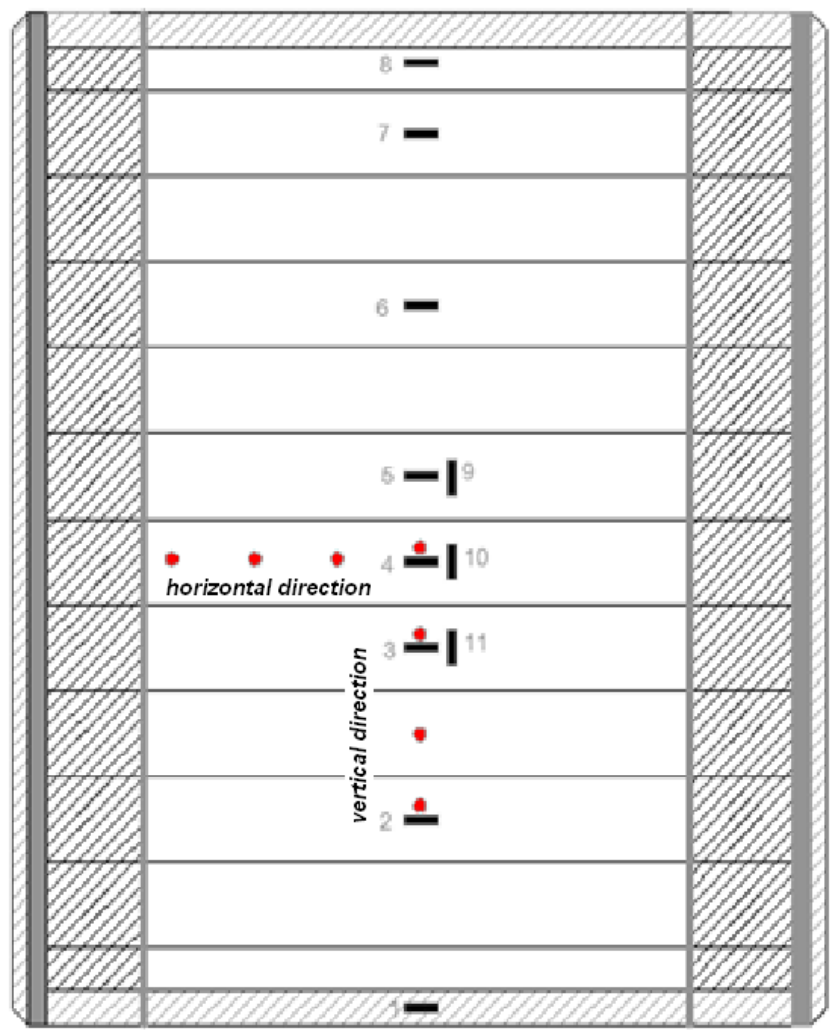

Figure C.6: Locations of strain gage (rectangle) and LVDTs (circle) on the back side of the panel

\section{C.4.1.1 With 6 in $x 6$ in plate placed at the center beam}

The load was applied through 6 in x 6 in plate (represented as a square in Figure C.7), placed at the center of the seventh hexagonal FRP beam (Figure C.3). The maximum load applied was 30.1 kip. Deflections as well as strains measured at various locations are shown in Table C-1 and C-2. Figure C.8 shows the plot of load vs. deflections.

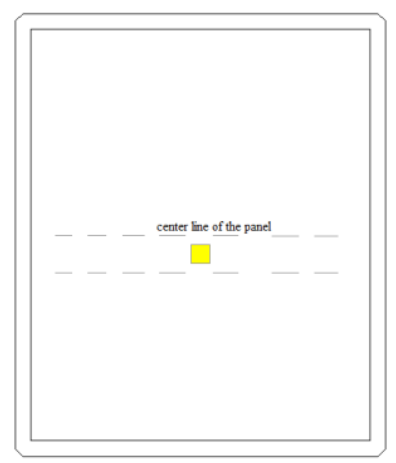

Figure C.7: Loading with 6 in $\times 6$ in plate 


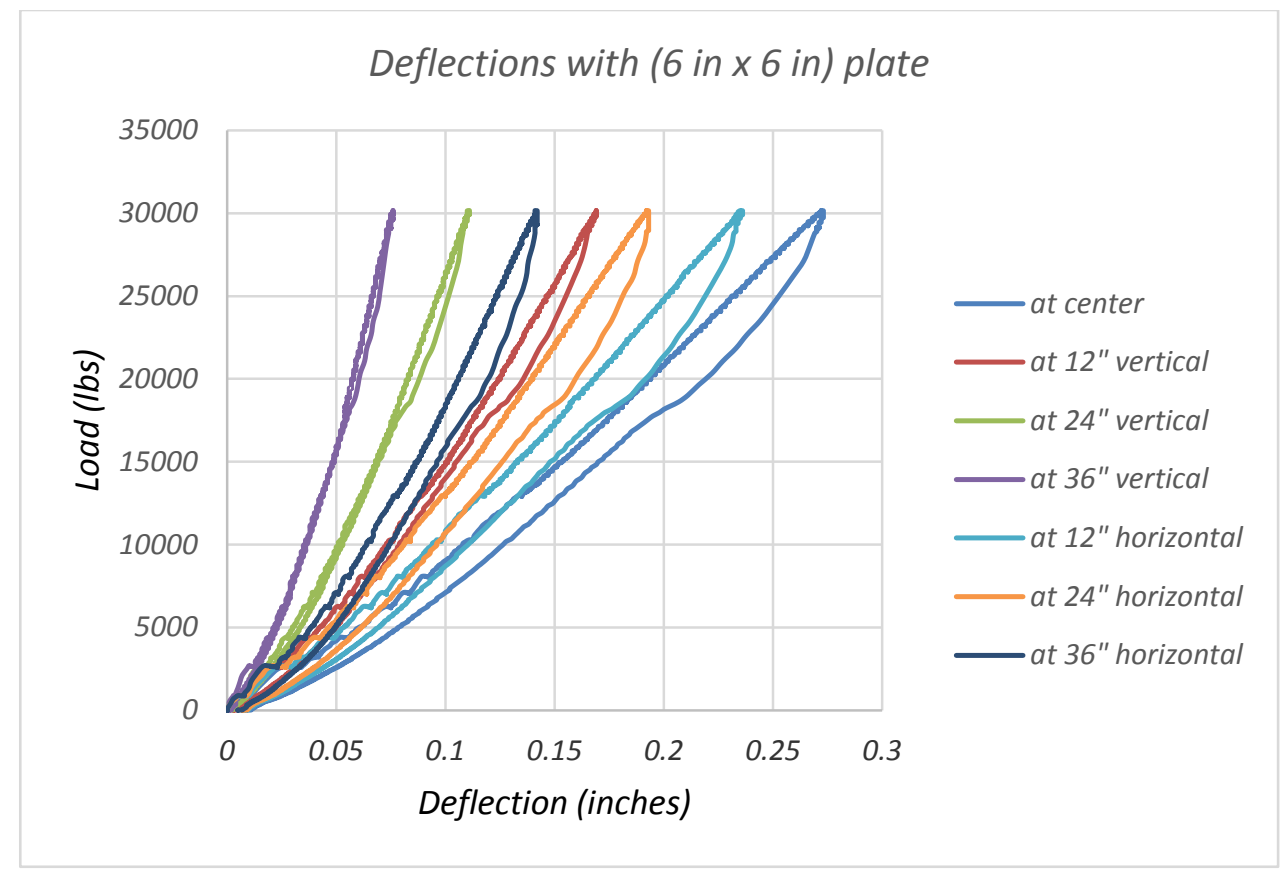

Figure C.8: Load vs. deflections plot

Table C-1: Load vs deflection

\begin{tabular}{|c|c|c|c|c|c|c|c|}
\hline \multirow{3}{*}{$\begin{array}{l}\text { Max. } \\
\text { Load } \\
\text { (kip) }\end{array}$} & \multirow{3}{*}{$\begin{array}{l}\text { Deflection } \\
\text { at center of } \\
\text { the load } \\
\text { position }\end{array}$} & \multicolumn{6}{|c|}{$\begin{array}{l}\text { Deflections away from load position in } \\
\text { (Figure C.6) }\end{array}$} \\
\hline & & \multicolumn{3}{|c|}{ horizontal direction } & \multicolumn{3}{|c|}{ vertical direction } \\
\hline & & @12” & @24" & $@ 36 ”$ & @12” & @24" & @36" \\
\hline 30.1 & 0.272 & 0.169 & 0.111 & 0.076 & 0.235 & 0.192 & 0.141 \\
\hline
\end{tabular}

Table C-2: Load vs strains

\begin{tabular}{|c|c|c|c|c|c|c|c|c|c|c|c|c|c|c|c|c|}
\hline $\begin{array}{c}\text { Max. } \\
\text { Load } \\
\text { (kip) }\end{array}$ & \multicolumn{10}{|c|}{ Micro-Strain at strain number position (Figure C.5 \& C.6) } \\
\hline 30.1 & 32 & 12 & 3 & 4 & 5 & 6 & 7 & 8 & 9 & 10 & 11 & 12 & 13 & 14 & 15 & 16 \\
\hline
\end{tabular}

C.4.1.2 With 6 in $x 24$ in plate placed horizontally at the center beam

The load was applied through 6 in x 24 in plate (represented as rectangle in Figure C.9), placed horizontally at the center of the seventh hexagonal FRP beam (Figure C.3). The 
maximum load applied was 30 kip. Deflections as well as strains measured at various locations are shown in Table C-3 and C-4. Figure C.10 shows the plot of load vs. deflections.

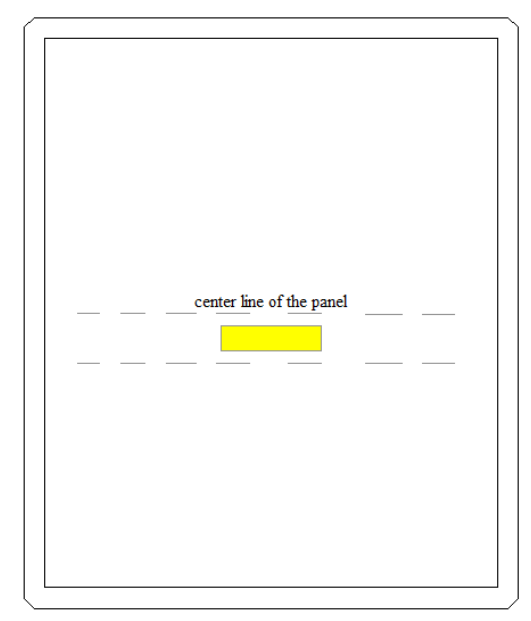

Figure C.9: Loading with the 6 in $x 24$ in plate placed horizontally

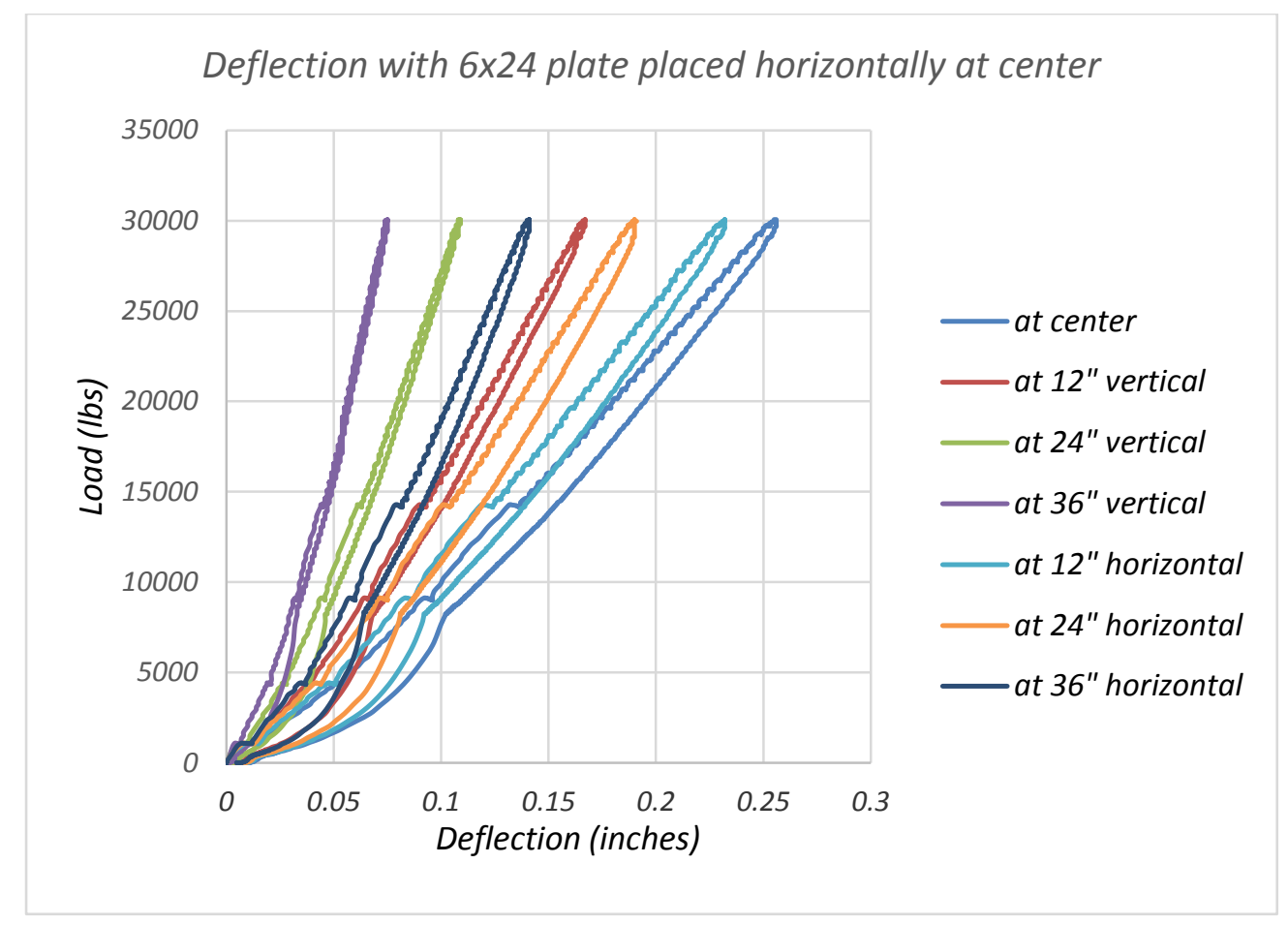

Figure C.10: Load vs. deflections 
Table C-3: Load vs. deflection

\begin{tabular}{|c|c|c|c|c|c|c|c|}
\hline \multirow{3}{*}{$\begin{array}{l}\text { Max. } \\
\text { Load } \\
\text { (kip) }\end{array}$} & \multirow{3}{*}{$\begin{array}{c}\text { Deflection } \\
\text { at center } \\
\text { of the } \\
\text { load } \\
\text { position }\end{array}$} & \multicolumn{6}{|c|}{$\begin{array}{c}\text { Deflections away from load position in } \\
\text { (Figure C.6) }\end{array}$} \\
\hline & & \multicolumn{3}{|c|}{ horizontal direction } & \multicolumn{3}{|c|}{ vertical direction } \\
\hline & & (a) $12 "$ & @24" & (a)36" & @12” & @24" & (a)36" \\
\hline 30 & 0.255 & 0.167 & 0.109 & 0.075 & 0.232 & 0.190 & 0.140 \\
\hline
\end{tabular}

Table C-4: Load vs. strains

\begin{tabular}{|c|c|c|c|c|c|c|c|c|c|c|c|c|c|c|c|c|}
\hline $\begin{array}{c}\text { Max. } \\
\text { Load } \\
\text { (kip) }\end{array}$ & \multicolumn{10}{|c|}{ Micro-Strain at strain number position (Figure C.5 and C.6) } \\
\cline { 2 - 17 } & 1 & 2 & 3 & 4 & 5 & 6 & 7 & 8 & 9 & 10 & 11 & 12 & 13 & 14 & 15 & 16 \\
\hline 30 & 31 & 126 & 383 & 625 & 405 & 126 & 45 & 31 & -182 & 525 & -210 & 54 & 21 & -436 & 138 & -66 \\
\hline
\end{tabular}

\section{C.4.1.3 With 6 in $x 24$ in plate placed vertically at the center beam}

The load was applied through 6 in $\mathrm{x} 24$ in plate (represented as a rectangle in Figure C.11), placed vertically at the center of the seventh hexagonal FRP beam (Figure C.3). The maximum load applied was $45 \mathrm{kip}$. Deflections as well as strains measured at various locations are shown in Table C-5 and C-6. Figure C.12 shows the plot of load vs. deflections.

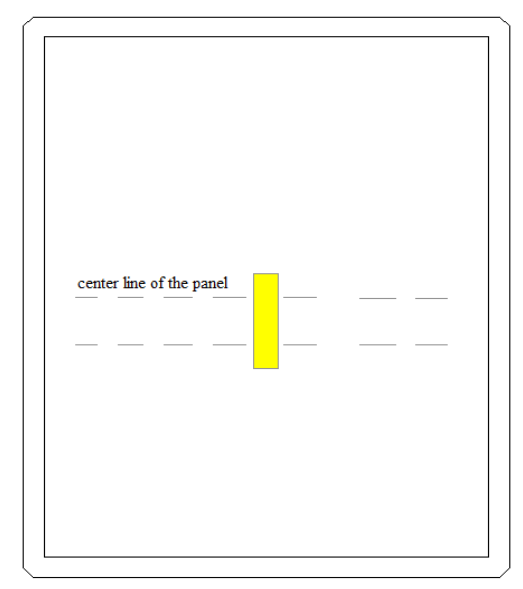

Figure C.11: Loading with the 6 in $x 24$ in plate placed vertically 


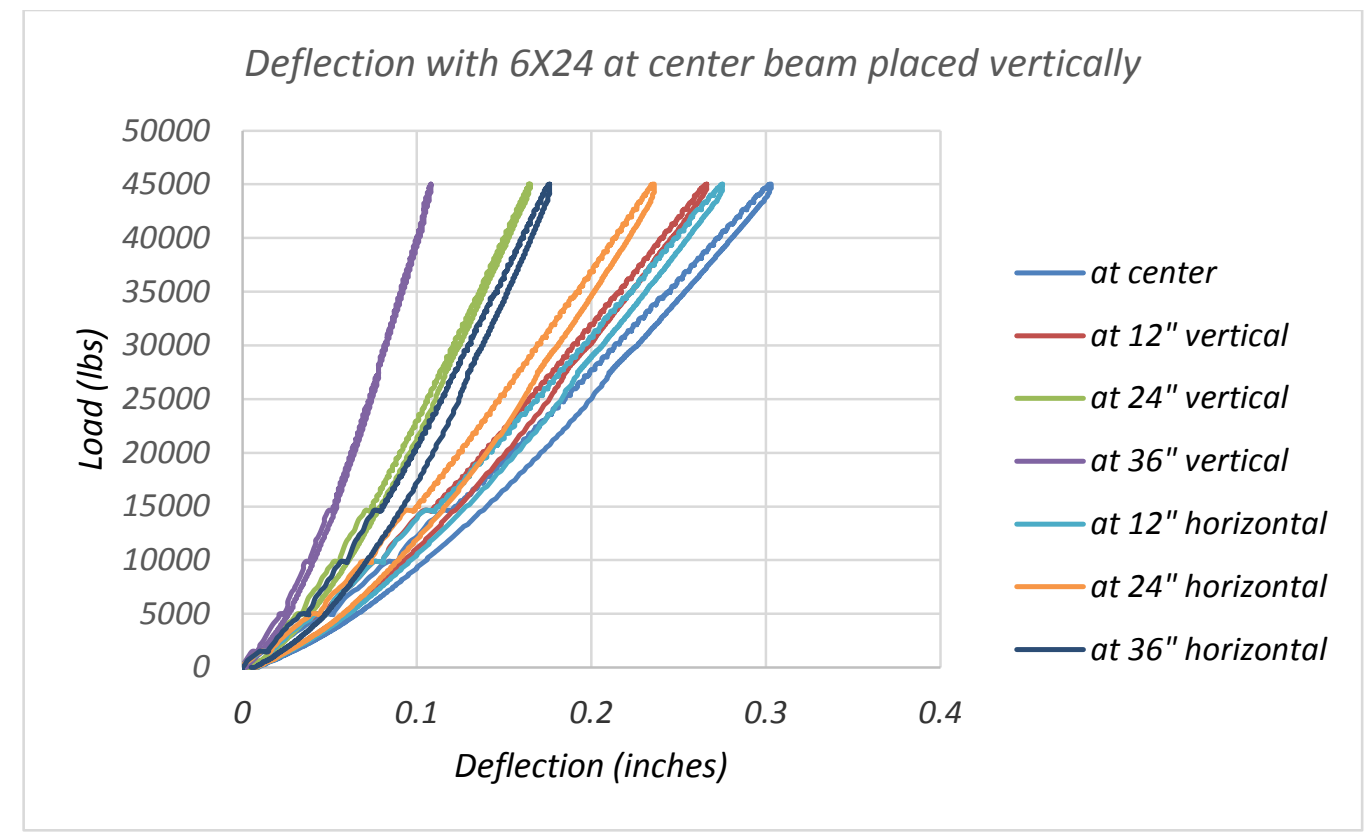

Figure C.12: Load vs. deflections plot

Table C-5: Load vs. deflections

\begin{tabular}{|c|c|c|c|c|c|c|c|}
\hline \multirow{3}{*}{$\begin{array}{l}\text { Max. } \\
\text { Load } \\
\text { (kip) }\end{array}$} & \multirow{3}{*}{$\begin{array}{c}\text { Deflection } \\
\text { at center } \\
\text { of the } \\
\text { load } \\
\text { position }\end{array}$} & \multicolumn{6}{|c|}{$\begin{array}{c}\text { Deflections away from load position in } \\
\text { (Figure C.6) }\end{array}$} \\
\hline & & \multicolumn{3}{|c|}{ horizontal direction } & \multicolumn{3}{|c|}{ vertical direction } \\
\hline & & (a) 12" & @24" & (a)36" & @ $12 "$ & @24" & @36" \\
\hline 45 & 0.303 & 0.266 & 0.164 & 0.108 & 0.275 & 0.235 & 0.176 \\
\hline
\end{tabular}

Table C-6: Load vs. strain

\begin{tabular}{|c|c|c|c|c|c|c|c|c|c|c|c|c|c|c|c|c|}
\hline \multirow{2}{*}{$\begin{array}{l}\text { Max. } \\
\text { Load } \\
\text { (kip) }\end{array}$} & \multicolumn{16}{|c|}{ Micro-Strain at strain number position (Figure C.5 and C.6) } \\
\hline & 1 & 2 & 3 & 4 & 5 & 6 & 7 & 8 & 9 & 10 & 11 & 12 & 13 & 14 & 15 & 16 \\
\hline 45 & 54 & 212 & 773 & 829 & 830 & 202 & 71 & 49 & 140 & -30 & 92 & 82 & 31 & -740 & 220 & -106 \\
\hline
\end{tabular}

\section{C.4.1.4 With 6 in $x 48$ in plate placed horizontally at the center beam}

The load was applied through 6 in $\mathrm{x} 48$ in plate (represented as a rectangle in Figure

C.13), placed horizontally at the center of the seventh hexagonal FRP beam (Figure C.3). The 
maximum load applied was 30 kip. Deflections as well as strains measured at various locations are shown in Table C.7 and C.8. Figure C.14 shows the plot of load vs. deflections.

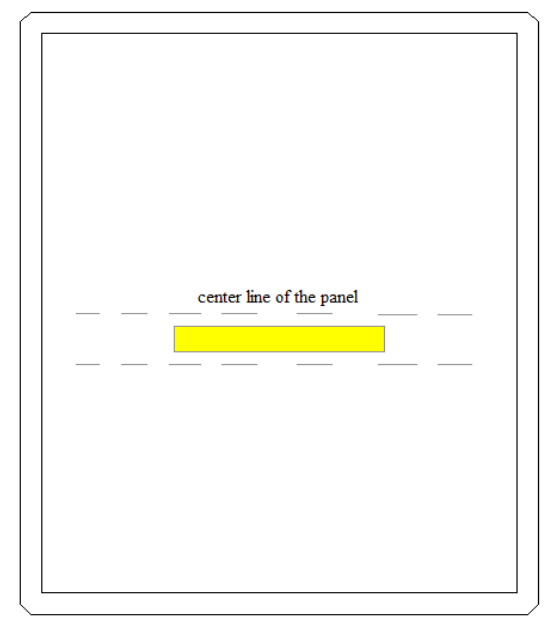

Figure C.13: Loading with the 6 in $x 48$ in plate placed horizontally

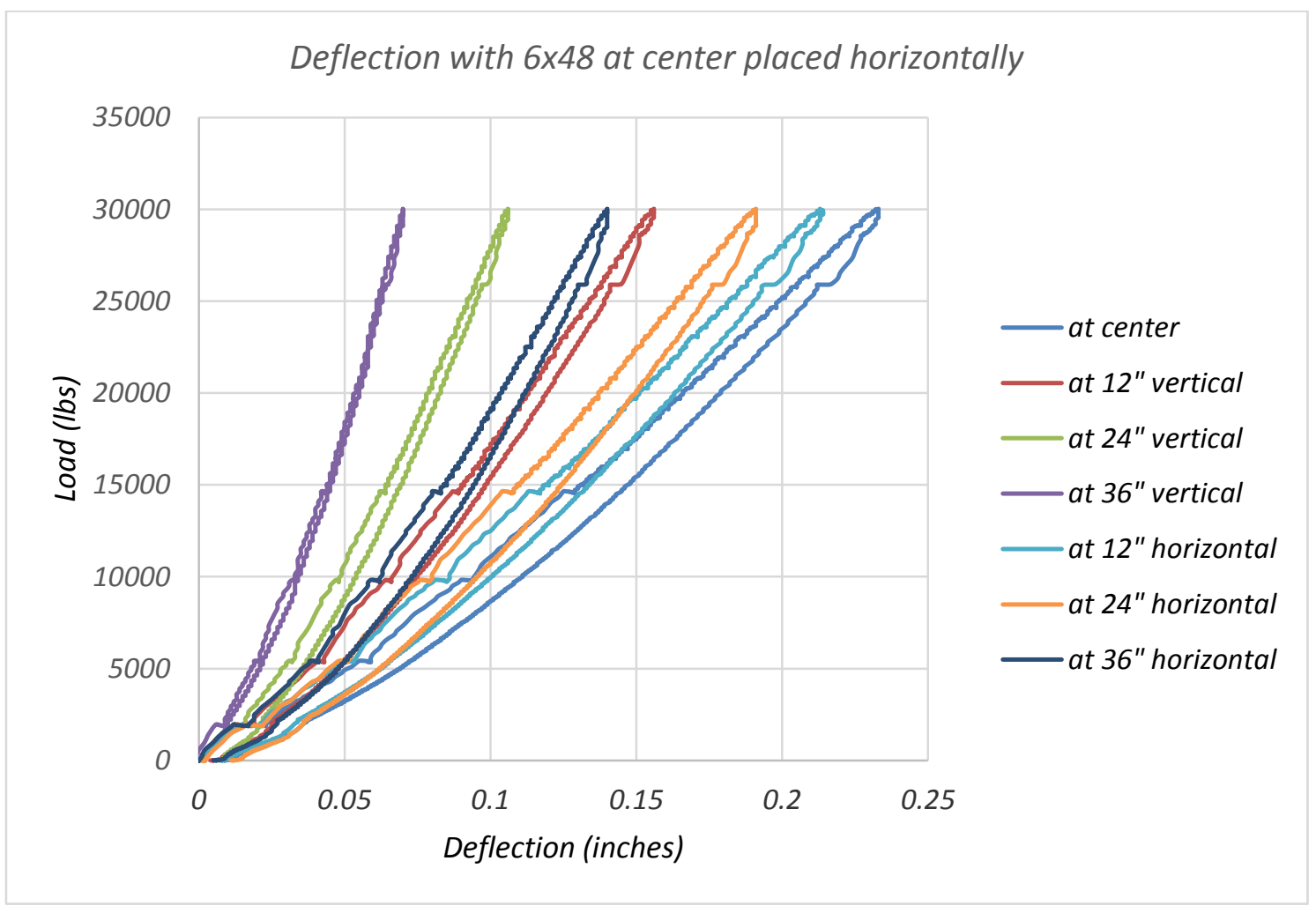

Figure C.14: Load vs. deflections plot 
Table C-7: Load vs. deflections

\begin{tabular}{|c|c|c|c|c|c|c|c|}
\hline \multirow{3}{*}{$\begin{array}{l}\text { Max. } \\
\text { Load } \\
\text { (kip) }\end{array}$} & \multirow{3}{*}{$\begin{array}{c}\text { Deflection } \\
\text { at center } \\
\text { of the } \\
\text { load } \\
\text { position }\end{array}$} & \multicolumn{6}{|c|}{$\begin{array}{l}\text { Deflections away from load position in } \\
\text { (Figure C.6) }\end{array}$} \\
\hline & & \multicolumn{3}{|c|}{ horizontal direction } & \multicolumn{3}{|c|}{ vertical direction } \\
\hline & & @12” & @ 24" & @36" & @12” & $@ 24 "$ & (a)36" \\
\hline 30 & 0.233 & 0.156 & 0.106 & 0.07 & 0.213 & 0.191 & 0.140 \\
\hline
\end{tabular}

Table C-8: Load vs. strains

\begin{tabular}{|c|c|c|c|c|c|c|c|c|c|c|c|c|c|c|c|c|}
\hline \multirow{2}{*}{$\begin{array}{l}\text { Max. } \\
\text { Load } \\
\text { (kip) }\end{array}$} & \multicolumn{16}{|c|}{ Micro-Strain at strain number position (Figure C.5 and C.6) } \\
\hline & 1 & 2 & 3 & 4 & 5 & 6 & 7 & 8 & 9 & 10 & 11 & 12 & 13 & 14 & 15 & 16 \\
\hline 30 & 29 & 119 & 327 & 479 & 351 & 121 & 42 & 29 & -140 & 457 & -157 & 54 & 21 & -338 & 127 & -62 \\
\hline
\end{tabular}

\section{C.4.1.5 With 6 in $x 48$ in plate placed vertically at the center beam}

The load was applied through 6 in $\mathrm{x} 48$ in plate (represented as a rectangle in Figure C.15), placed vertically at the center of the seventh hexagonal FRP beam (Figure C.3). The maximum load applied was $90 \mathrm{kip}$. Deflections as well as strains measured at various locations are shown in Table C-9 and C-10. Figure C.16 shows the plot of load vs. deflections.

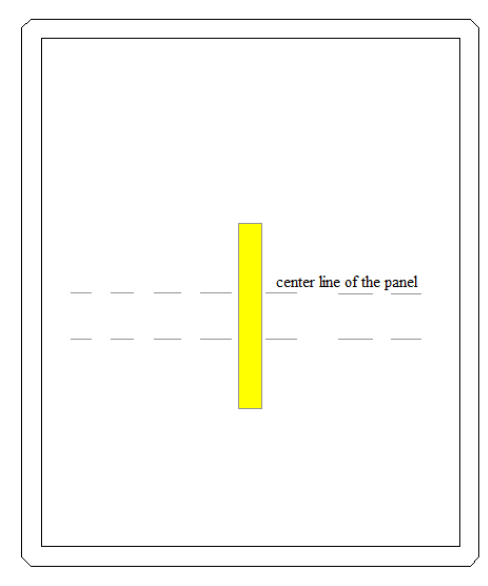

Figure C.15: Loading with the 6 in $x 48$ in plate placed vertically 


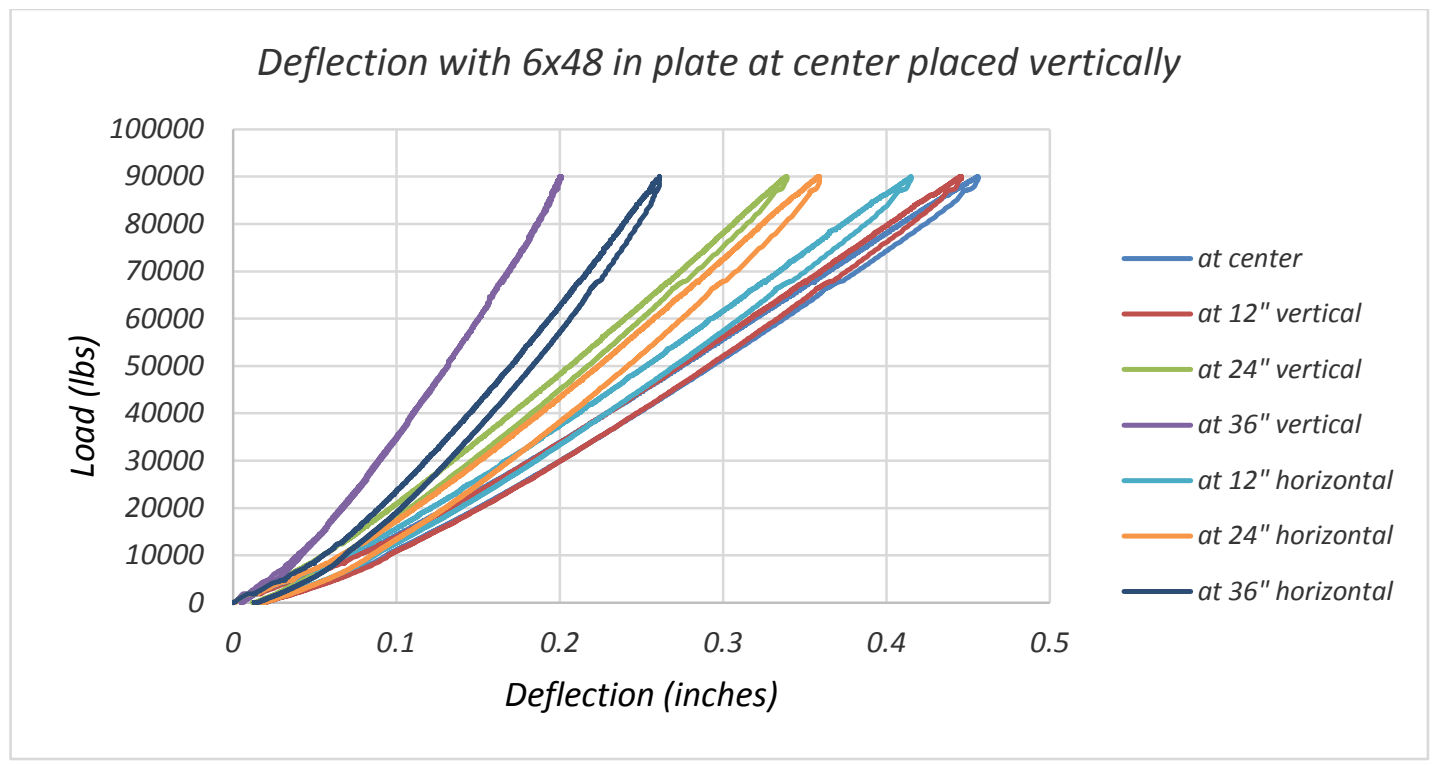

Figure C.16: Load vs. deflections plot

Table C-9: Load vs. deflections

\begin{tabular}{|c|c|c|c|c|c|c|c|}
\hline \multirow{3}{*}{$\begin{array}{l}\text { Max. } \\
\text { Load } \\
\text { (kip) }\end{array}$} & \multirow{3}{*}{$\begin{array}{c}\text { Deflection at } \\
\text { center of the } \\
\text { load } \\
\text { position }\end{array}$} & \multicolumn{6}{|c|}{ Deflections away from load position in (Figure C.6) } \\
\hline & & \multicolumn{3}{|c|}{ horizontal direction } & \multicolumn{3}{|c|}{ vertical direction } \\
\hline & & @12" & (a)24” & @36" & (a) $12 "$ & @24” & (a)36" \\
\hline 90 & 0.456 & 0.445 & 0.339 & 0.201 & 0.415 & 0.358 & 0.261 \\
\hline
\end{tabular}

Table C-10: Load vs. strains

\begin{tabular}{|c|c|c|c|c|c|c|c|c|c|c|c|c|c|c|c|c|}
\hline \multirow{2}{*}{$\begin{array}{l}\text { Max. } \\
\text { Load } \\
\text { (kip) }\end{array}$} & \multicolumn{16}{|c|}{ Micro-Strain at strain number position (Figure C.5 and C.6) } \\
\hline & 1 & 2 & 3 & 4 & 5 & 6 & 7 & 8 & 9 & 10 & 11 & 12 & 13 & 14 & 15 & 16 \\
\hline 90 & 113 & 461 & 1384 & 1296 & 1352 & 452 & 150 & 98 & 29 & -171 & 28 & 119 & 46 & -2883 & 451 & -229 \\
\hline
\end{tabular}

\section{C.4.2 Deflections measured only in one direction (vertical) as in Fig. C.3}

In this testing, deflections were measured in only one direction of the panel as shown in Figure C.18. Strain gage locations as well as LVDT locations on top, front, side and bottom face of the panel is shown in Figure C.17 and C.18. All the locations have been numbered for consistency and ease to present the test results. The rectangle represents the strain gage whereas 
the circle represents the LVDTs. The test results shown within this section correspond to the locations of strain gages and LVDTs. The following testing has been done with deflections measured in one direction.

i. $\quad$ With 6 in $x 6$ in plate placed at the center of the ninth beam (Fig. C.3)

ii. $\quad$ With 6 in $x 6$ in plate placed at the center beam (Fig. C.3)

iii. $\quad$ With 6 in $x 24$ in plate placed horizontally at the center beam (Fig. C.3)

iv. $\quad$ With 6 in $x 24$ in plate placed vertically at the center beam (Fig. C.3)

v. With 6 in $x 48$ in plate placed horizontally at the center beam (Fig. C.3)

vi. $\quad$ With 6 in $x 48$ in plate placed vertically at the center beam (Fig. C.3)

NOTE: Remaining five test results has been used to determine the load distribution factor for the panel and is provided in the next section C.3.

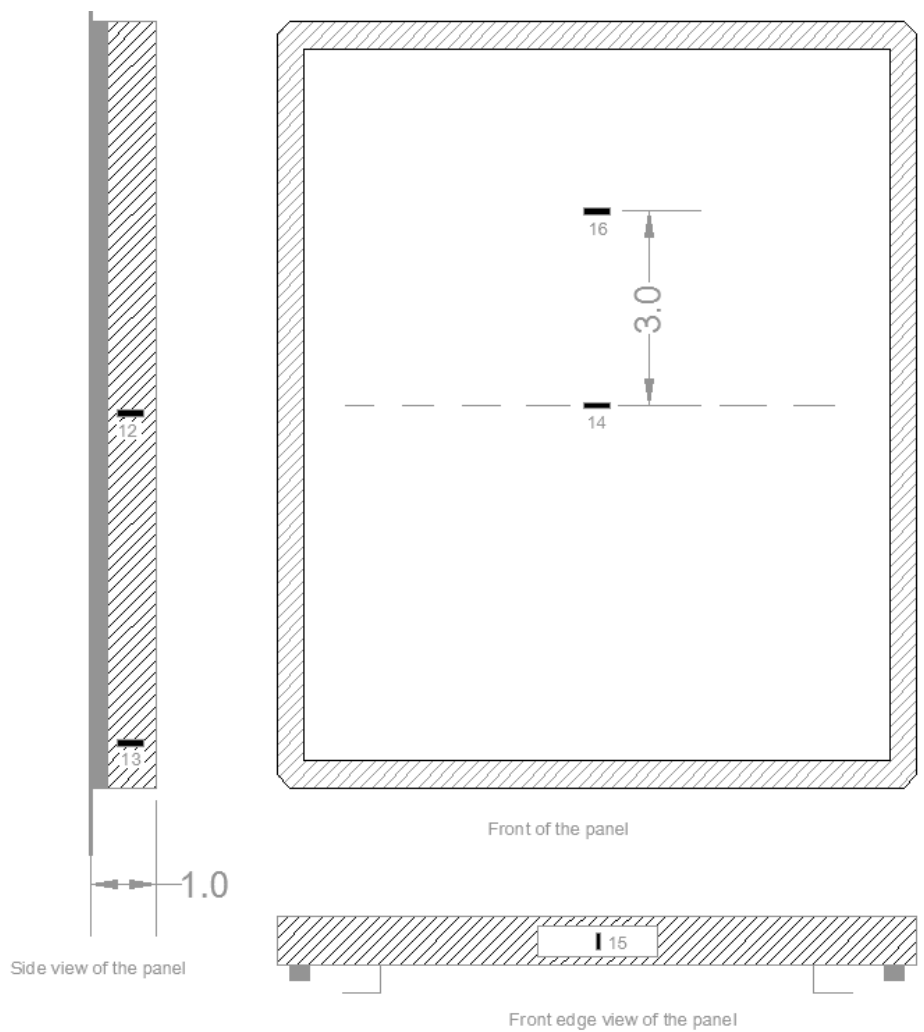

Figure C.17: Locations of strain gage (rectangle) on top, front and side of the panel 


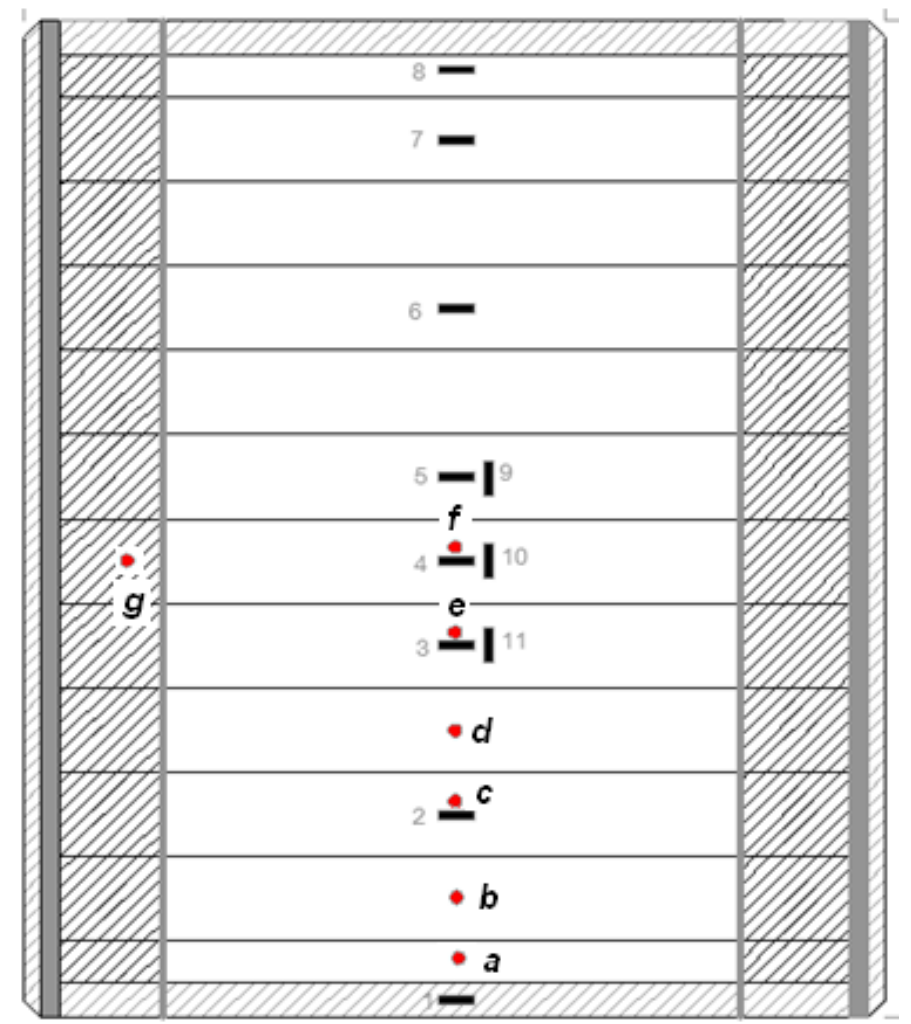

Figure C.18: Locations of strain gages (rectangle) and LVDTs (circle) on the back of the panel

\section{C.4.2.1 With 6 in $x 6$ in plate placed at the center of the $9^{\text {th }}$ beam}

The load was applied through 6 in x 6 in plate (Figure C.19), placed at the center of the ninth hexagonal FRP beam (Figure C.3). The maximum load applied was 18 kip. Deflections as well as strains measured at various locations are shown in Table C-11 and C-12.

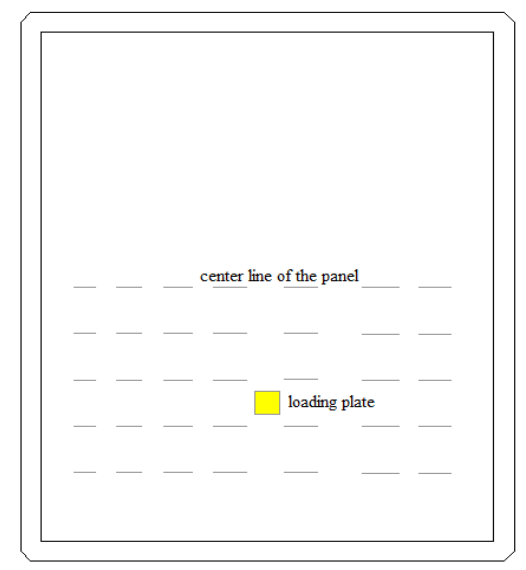

Figure C.16: Loading with the 6 in $x 6$ in plate placed at the center of the $9^{\text {th }}$ beam 
Table C-11: Load vs. deflections

\begin{tabular}{|c|c|c|c|c|c|c|c|}
\hline \multirow{2}{*}{$\begin{array}{c}\text { Max. } \\
\text { Load } \\
\text { (kip) }\end{array}$} & \multicolumn{7}{|c|}{ Deflection at different position (Figure C.18) } \\
\cline { 2 - 8 } & $\mathrm{a}$ & $\mathrm{b}$ & $\mathrm{c}$ & $\mathrm{d}$ & $\mathrm{e}$ & $\mathrm{f}$ & $\mathrm{g}$ \\
\hline 18 & 0.083 & 0.093 & 0.114 & 0.165 & 0.105 & 0.068 & 0.026 \\
\hline
\end{tabular}

Table C-12: Load vs. strains

\begin{tabular}{|c|c|c|c|c|c|c|c|c|c|c|c|c|c|c|c|c|}
\hline \multirow{2}{*}{$\begin{array}{l}\text { Max. } \\
\text { Load } \\
\text { (kip) }\end{array}$} & \multicolumn{16}{|c|}{ Micro-Strain at strain number position (Figure C.17 \& C.18) } \\
\hline & 1 & 2 & 3 & 4 & 5 & 6 & 7 & 8 & 9 & 10 & 11 & 12 & 13 & 14 & 15 & 16 \\
\hline 18 & 55 & 231 & 217 & 106 & 67 & 29 & 386 & 6 & -11 & -7 & -140 & 2 & 9 & -55 & 451 & 229 \\
\hline
\end{tabular}

\section{C.5 Load distribution factors}

The deflections and strains measured from the bottom flanges of twelve FRP superdecks, constituting the recess panel, have been analyzed to compute the load distribution factors (LDF) for the panel. LDF is the deflection/strain measured on particular hexagonal section under the centroid of the load divided by the total deflections/strains of all other adjacent hexagonal sections (Tables C-13 to C-17). The results obtained from following testing were used for the computation of LDF:

i. $\quad$ With 6 in $x 6$ in plate placed at the center beam (Fig. C.3)

ii. $\quad$ With 6 in $x 24$ in plate placed horizontally at the center beam (Fig. C.3)

iii. $\quad$ With 6 in $x 24$ in plate placed vertically at the center beam (Fig. C.3)

iv. $\quad$ With 6 in $x 48$ in plate placed horizontally at the center beam (Fig. C.3)

v. With 6 in $x 48$ in plate placed vertically at the center beam (Fig. C.3) 
Table C-13: With 6in x 6in plate placed on the center beam (\#7) (@ 30 kip load)

\begin{tabular}{|c|c|c|c|c|c|c|}
\hline \multirow{2}{*}{ Beam no. } & \multicolumn{2}{|c|}{ With respect to deflections } & \multicolumn{3}{c|}{ With respect to strains } \\
\cline { 2 - 7 } & Deflection & LDF & LDF (\%) & Strain & LDF & LDF (\%) \\
\hline 1 & 0.035 & 0.026882 & 2.69 & 28 & 0.01059 & 1.06 \\
\hline 2 & 0.052 & 0.039939 & 3.99 & 43 & 0.016263 & 1.63 \\
\hline 3 & 0.066 & 0.050691 & 5.07 & 85 & 0.032148 & 3.21 \\
\hline 4 & 0.083 & 0.063748 & 6.37 & 127 & 0.048033 & 4.80 \\
\hline 5 & 0.117 & 0.089862 & 8.99 & 251 & 0.094932 & 9.49 \\
\hline 6 & 0.173 & 0.132873 & 13.29 & 376 & 0.142209 & 14.22 \\
\hline 7 & 0.285 & 0.218894 & 21.89 & 852 & 0.322239 & 32.22 \\
\hline 8 & 0.173 & 0.132873 & 13.29 & 376 & 0.142209 & 14.22 \\
\hline 9 & 0.117 & 0.089862 & 8.99 & 251 & 0.094932 & 9.49 \\
\hline 10 & 0.083 & 0.063748 & 6.37 & 127 & 0.048033 & 4.80 \\
\hline 11 & 0.066 & 0.050691 & 5.07 & 85 & 0.032148 & 3.21 \\
\hline 12 & 0.052 & 0.039939 & 3.99 & 43 & 0.016263 & 1.63 \\
\hline & $\sum=1.302$ & & & $\sum=2644$ & & \\
\hline
\end{tabular}

Table C-14: With 6in x 24in plate placed horizontally on the center beam (\#7) (@30 kip load)

\begin{tabular}{|c|c|c|c|c|c|c|}
\hline \multirow{2}{*}{ Beam no. } & \multicolumn{2}{|c|}{ With respect to deflections } & \multicolumn{2}{c|}{ With respect to strains } \\
\cline { 2 - 7 } & Deflection & LDF & LDF (\%) & Strain & LDF & LDF (\%) \\
\hline 1 & 0.032 & 0.026644 & 2.66 & 32 & 0.012413 & 1.24 \\
\hline 2 & 0.045 & 0.037469 & 3.75 & 47 & 0.018231 & 1.82 \\
\hline 3 & 0.059 & 0.049126 & 4.91 & 90 & 0.034911 & 3.49 \\
\hline 4 & 0.077 & 0.064113 & 6.41 & 134 & 0.051978 & 5.20 \\
\hline 5 & 0.11 & 0.09159 & 9.16 & 273 & 0.105896 & 10.59 \\
\hline 6 & 0.165 & 0.137386 & 13.74 & 412 & 0.159814 & 15.98 \\
\hline 7 & 0.257 & 0.213988 & 21.40 & 634 & 0.245927 & 24.59 \\
\hline 8 & 0.165 & 0.137386 & 13.74 & 412 & 0.159814 & 15.98 \\
\hline 9 & 0.11 & 0.09159 & 9.16 & 273 & 0.105896 & 10.59 \\
\hline 10 & 0.077 & 0.064113 & 6.41 & 134 & 0.051978 & 5.20 \\
\hline
\end{tabular}




\begin{tabular}{|c|c|c|c|c|c|c|}
\hline 11 & 0.059 & 0.049126 & 4.91 & 90 & 0.034911 & 3.49 \\
\hline 12 & 0.045 & 0.037469 & 3.75 & 47 & 0.018231 & 1.82 \\
\hline & $\sum=1.201$ & & & $\sum=2578$ & & \\
\hline
\end{tabular}

Table C-15: With 6in x 24in plate placed vertically on the center beam (\#7) (@60 kip load)

\begin{tabular}{|c|c|c|c|c|c|c|}
\hline \multirow{2}{*}{ Beam no. } & \multicolumn{2}{|c|}{ With respect to deflections } & \multicolumn{3}{c|}{ With respect to strains } \\
\cline { 2 - 7 } & Deflection & LDF & LDF (\%) & Strain & LDF & LDF (\%) \\
\hline 1 & 0.052 & 0.023561 & 2.36 & 62 & 0.011414 & 1.14 \\
\hline 2 & 0.079 & 0.035795 & 3.58 & 91 & 0.016753 & 1.68 \\
\hline 3 & 0.103 & 0.04667 & 4.67 & 140 & 0.025773 & 2.58 \\
\hline 4 & 0.142 & 0.064341 & 6.43 & 286 & 0.052651 & 5.27 \\
\hline 5 & 0.209 & 0.094699 & 9.47 & 540 & 0.099411 & 9.94 \\
\hline 6 & 0.35 & 0.158586 & 15.86 & 1074 & 0.197717 & 19.77 \\
\hline 7 & 0.389 & 0.176257 & 17.63 & 1108 & 0.203976 & 20.40 \\
\hline 8 & 0.35 & 0.158586 & 15.86 & 1074 & 0.197717 & 19.77 \\
\hline 9 & 0.209 & 0.094699 & 9.47 & 540 & 0.099411 & 9.94 \\
\hline 10 & 0.142 & 0.064341 & 6.43 & 286 & 0.052651 & 5.27 \\
\hline 11 & 0.103 & 0.04667 & 4.67 & 140 & 0.025773 & 2.58 \\
\hline 12 & 0.079 & 0.035795 & 3.58 & 91 & 0.016753 & 1.68 \\
\hline & $\sum=2.207$ & & & $\sum=5432$ & & \\
\hline
\end{tabular}

Table C-16: With 6in x 48in plate placed horizontally on the center beam (\#7) (@ 30.7 kip load)

\begin{tabular}{|c|c|c|c|c|c|c|}
\hline \multirow{2}{*}{ Beam no. } & \multicolumn{2}{|c|}{ With respect to deflections } & \multicolumn{3}{c|}{ With respect to strains } \\
\cline { 2 - 7 } & Deflection & LDF & LDF (\%) & Strain & LDF & LDF (\%) \\
\hline 1 & 0.031 & 0.027679 & 2.77 & 31 & 0.014595 & 1.46 \\
\hline 2 & 0.044 & 0.039286 & 3.93 & 45 & 0.021186 & 2.12 \\
\hline 3 & 0.057 & 0.050893 & 5.09 & 75 & 0.035311 & 3.53 \\
\hline 4 & 0.073 & 0.065179 & 6.52 & 124 & 0.05838 & 5.84 \\
\hline 5 & 0.103 & 0.091964 & 9.20 & 222 & 0.10452 & 10.45 \\
\hline
\end{tabular}




\begin{tabular}{|c|c|c|c|c|c|c|}
\hline 6 & 0.153 & 0.136607 & 13.66 & 340 & 0.160075 & 16.01 \\
\hline 7 & 0.229 & 0.204464 & 20.45 & 481 & 0.22646 & 22.65 \\
\hline 8 & 0.153 & 0.136607 & 13.66 & 340 & 0.160075 & 16.01 \\
\hline 9 & 0.103 & 0.091964 & 9.20 & 222 & 0.10452 & 10.45 \\
\hline 10 & 0.073 & 0.065179 & 6.52 & 124 & 0.05838 & 5.84 \\
\hline 11 & 0.057 & 0.050893 & 5.09 & 75 & 0.035311 & 3.53 \\
\hline 12 & 0.044 & 0.039286 & 3.93 & 45 & 0.021186 & 2.12 \\
\hline & $\sum=1.12$ & & & $\sum=2124$ & & \\
\hline
\end{tabular}

Table C-17: With 6in x 48in plate vertically on the center beam (\#7) (@60.2 kip load)

\begin{tabular}{|c|c|c|r|c|c|r|}
\hline \multirow{2}{*}{ Beam no. } & \multicolumn{2}{|c|}{ With respect to deflections } & \multicolumn{3}{c|}{ With respect to strains } \\
\cline { 2 - 7 } & Deflection & LDF & LDF (\%) & Strain & LDF & LDF (\%) \\
\hline 1 & 0.05 & 0.023474 & 2.35 & 74 & 0.013568 & 1.36 \\
\hline 2 & 0.08 & 0.037559 & 3.76 & 108 & 0.019802 & 1.98 \\
\hline 3 & 0.107 & 0.050235 & 5.02 & 205 & 0.037587 & 3.76 \\
\hline 4 & 0.148 & 0.069484 & 6.95 & 320 & 0.058673 & 5.87 \\
\hline 5 & 0.228 & 0.107042 & 10.70 & 678 & 0.124312 & 12.43 \\
\hline 6 & 0.313 & 0.146948 & 14.69 & 940 & 0.172351 & 17.24 \\
\hline 7 & 0.328 & 0.153991 & 15.40 & 878 & 0.160983 & 16.10 \\
\hline 8 & 0.313 & 0.146948 & 14.69 & 940 & 0.172351 & 17.24 \\
\hline 9 & 0.228 & 0.107042 & 10.70 & 678 & 0.124312 & 12.43 \\
\hline 10 & 0.148 & 0.069484 & 6.95 & 320 & 0.058673 & 5.87 \\
\hline 11 & 0.107 & 0.050235 & 5.02 & 205 & 0.037587 & 3.76 \\
\hline 12 & 0.08 & 0.037559 & 3.76 & 108 & 0.019802 & 1.98 \\
\hline & $\sum=2.207$ & & & $\sum=5432$ & & \\
\hline
\end{tabular}




\section{C.6 Conclusions}

FRP panel supported on two edges similar to the field installation was tested under the application of square load (6" x 6") and rectangular patch loads (6" x 24", 6" x48") along horizontal and vertical directions. Based on measured deflections and strains for all loading conditions, maximum fraction of the load taken by the beam directly under the centroid of the load was $\sim 22 \%$. Remaining percentage of the load was distributed to several other beams throughout the panel. However, lower strain values in case of a smaller patch load (6" x 6") indicate that stresses in the beam directly under the centroid of the load may be little higher as indicated by $32 \%$ strain in that beam (Table C-13). Results indicate that the adhesive bonding is an effective tool in building a single panel from a group of hexagonal beams.

The recess panel made with twelve FRP superdecks bonded with shear connecters and "Rhino liner" coating was able to distribute the load effectively throughout the panel. The load applied on a single beam was effectively distributed to several other beams increasing the amount of energy absorption and the spreading of impact forces. The failure load for the single beam of the panel directly under the applied load is estimated to be approximately 4.5 times greater than its individual capacity. 